\title{
Preparation and characterization of polyimide/organoclay nanocomposites
}

Donavon Mark Delozier

College of William \& Mary - Arts \& Sciences

Follow this and additional works at: https://scholarworks.wm.edu/etd

Part of the Polymer Chemistry Commons

\section{Recommended Citation}

Delozier, Donavon Mark, "Preparation and characterization of polyimide/organoclay nanocomposites" (2002). Dissertations, Theses, and Masters Projects. Paper 1539623403.

https://dx.doi.org/doi:10.21220/s2-zpcf-8t73

This Dissertation is brought to you for free and open access by the Theses, Dissertations, \& Master Projects at W\&M ScholarWorks. It has been accepted for inclusion in Dissertations, Theses, and Masters Projects by an authorized administrator of W\&M ScholarWorks. For more information, please contact scholarworks@wm.edu. 


\section{INFORMATION TO USERS}

This manuscript has been reproduced from the microfilm master. UMI films the text directly from the original or copy submitted. Thus, some thesis and dissertation copies are in typewriter face, while others may be from any type of computer printer.

The quality of this reproduction is dependent upon the quality of the copy submitted. Broken or indistinct print, colored or poor quality illustrations and photographs, print bleedthrough, substandard margins, and improper alignment can adversely affect reproduction.

In the unlikely event that the author did not send UMI a complete manuscript and there are missing pages, these will be noted. Also, if unauthorized copyright material had to be removed, a note will indicate the deletion.

Oversize materials (e.g., maps, drawings, charts) are reproduced by sectioning the original, beginning at the upper left-hand corner and continuing from left to right in equal sections with small overlaps.

ProQuest Information and Learning 300 North Zeeb Road, Ann Arbor, MI 48106-1346 USA 800-521-0600

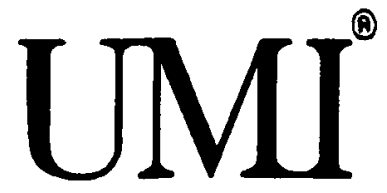


Reproduced with permission of the copyright owner. Further reproduction prohibited without permissiom. 


\title{
Preparation and Characterization of Polyimide/Organoclay Nanocomposites
}

\author{
A Dissertation \\ Presented to \\ The Faculty of the Department of Applied Science
}

\author{
In Partial Fulfillment \\ of the Requirements for the Degree of \\ Doctor of Philosophy
}

by

Donavon Delozier

2002 
This dissertation is submitted in partial fulfillment of

the requirements for the degree of

Doctor of Philosophy

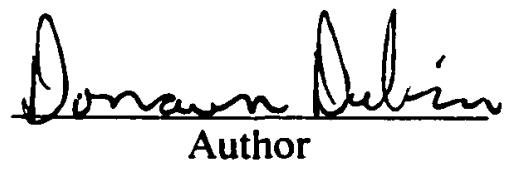

Approved, June 2002
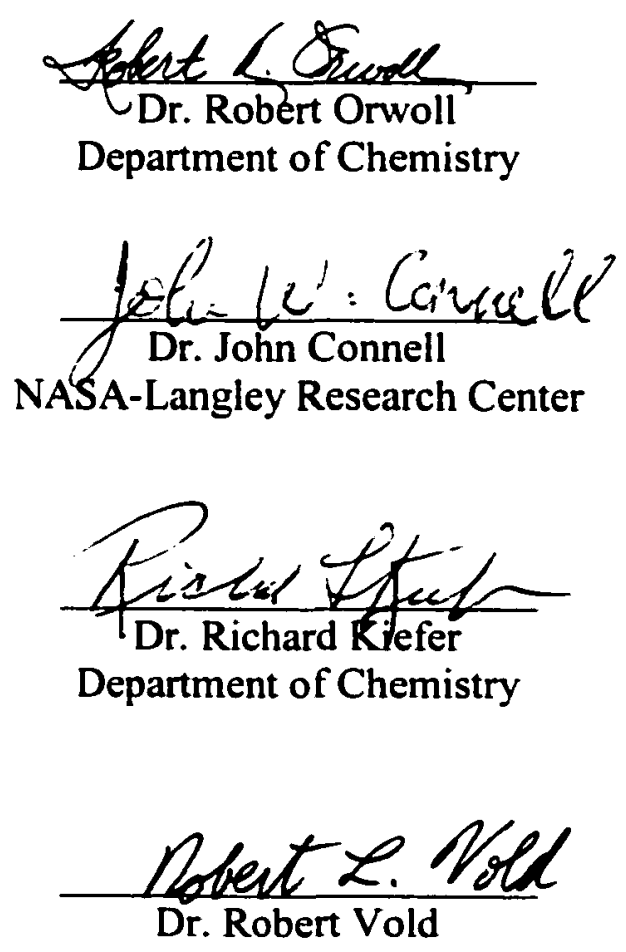


$\begin{array}{lr} & \text { Page } \\ \text { ACKNOWLEDGEMENTS } & \text { iv } \\ \text { LIST OF TABLES } & \mathrm{v} \\ \text { LIST OF FIGURES } & \mathrm{vi} \\ \text { ABSTRACT } & \mathrm{xii} \\ \text { CHAPTER I. INTRODUCTION } & 2 \\ \text { CHAPTER II. SIMPLE MIXING } & 26 \\ \text { CHAPTER III. IN-SITU POLYMERIZATION WITH LOW TEMPERATURE } & 62 \\ \text { ORGANOCLAYS } & 102 \\ \text { CHAPTER IV. IN-SITU POLYMERIZATION WITH HIGH TEMPERATURE } & \\ \text { ORGANOCLAYS } & 175 \\ \text { CHAPTER V. IN-SITI POLYMERIZATION WITH CHARGE OPTIMIZED, HIGH } \\ \text { TEMPERATURE ORGANOCLAYS } \\ \text { CHAPTER VI. CONCLUSION } & 238\end{array}$




\section{ACKNOWLEDGEMENTS}

The writer wishes to express his appreciation to the many people who have contributed to this work. Dr. Norman Johnston at NASA-LaRC provided the idea for the project as well as guidance and leadership of the project in the first year. Dr. John Connell and Dr. Joseph Smith assumed the NASA advisory role after Dr. Johnston's retirement and were extremely helpful. They each played vital roles in providing direction and providing training in lab practices. Dr. Orwoll has served as my advisor at William and Mary for the entire project and has provided invaluable insight into the project as well as practical guidance in program management. The writer would also like to thank the undergraduates who assisted in the project including James Cahoon, Bella Korik, Janine Ladislaw, and Chris Fox. The writer is greatly indebted to Jewel Thomas in the Biology department at the College of William and Mary for the countless hours she spent collecting TEM data. She and Dr. Scott also spent a great deal of time and energy patiently teaching the writer to prepare samples and use the microscope. Dr. Catherine Chisolm-Brause was very helpful in training the writer to use the $\mathrm{x}$-ray diffractometer at The Virginia Institute of Marine Science.

The writer would like to especially thank Dr. Orwoll for his aid in preparing this dissertation and for also serving on my defense committee. The writer also thanks Dr. Connell, Dr. Keifer, and Dr. Vold for their reading and criticism of the manuscript as well as serving on my defense committee. 


\section{LIST OF TABLES}

Table

Chapter 2

1. Calculation of mononomer addition amounts for controlled MW polyimide

2. Mechanical properties of PPD organoclay/ODA-PMDA hybrids

3. Mechanical properties of PPD organoclay/ODA-PMDA(offset) hybrid

4. Mechanical properties of hectorite PPD/ODA-PMDA hybrid

5. Mechanical properties of TPP organoclay/ODA-PMDA hybrid films

\section{Chapter 3}

1. Effectiveness of SCPX-2003 in polyimide nanocomposites

Chapter 4

1. Description of abbreviations

2. Visual analysis rating system

3. Calculated d-spacings for organoclays alone and in hybrid films

4. Visual analysis of polyimide films

170

Chapter 5

1. Description of abbreviations

2. TGA $5 \%$ weight loss values of organoclays

3. CEC values of MCM clays 


\section{LIST OF FIGURES}

Figure

Chapter 1

$\begin{array}{ll}\text { 1. Mechanism of PAA formation } & 6\end{array}$

2. 3-D representation of montmorillonite clay $\quad 8$

$\begin{array}{ll}\text { 3. Diagram of ion exchange process } & 10\end{array}$

4. Possible hybrid morphologies $\quad 11$

5. TEM of a disordered PMMA nanocomposite material $\quad 14$

6. TEM of a polysulfone/organoclay nanocomposite 15

7. TEM micrograph of a polypropylene/montmorillonite nanocomposite material 15

8. TEM micrographs of polysulfone/MMT nanocomposites at 1,3 , and $5 \%$

9. TEM of polystyrene/montmorillonite nanocomposite 17

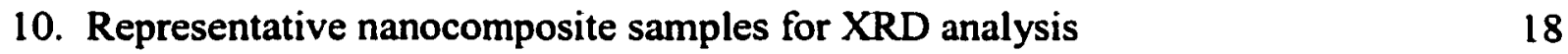

Chapter 2

1. Structure of ions used for ion exchange 37

2. Flow chart of simple mixing technique $\quad 39$

3. Chemical structures of monomers, PAA's, and polyimides 41

4. XRD pattern of SCPX-2003 organoclay 50

5. XRD pattern of homogenized SCPX-2003/ODA-BTDA hybrids 51

6. TEM micrograph of a 3\% SCPX-2003/ODA-BTDA polyimide prepared via homogenization $\quad 52$

7. XRD pattern of simply mixed SCPX-2003/ODA-BTDA nanocomposites 54 
8. TEM micrograph of 3\% SCPX-2003/ODA-BTDA hybrid film prepared via simple mixing

9. Modulus of SCPX-2003/ODA-BTDA (15000 g/mol) hybrids

10. Strength of SCPX-2003/ODA-BTDA (15000 g/mol) hybrids

11. Elongation of SCPX-2003/ODA-BTDA $(15000 \mathrm{~g} / \mathrm{mol})$ hybrids

\section{Chapter 3}

1. Structures of surfactant ions

2. Flow chart of hybrid preparation $\quad 69$

3. Monomers used to prepare polyimides 71

4. Temperature of $5 \%$ weight loss in air versus clay loading 76

5. TGA of quaternary tallow ammonium organoclay in air and nitrogen 77

6. TEM micrograph of $3 \%$ clay APB-BPDA films showing exfoliated clays 79

7. TEM micrograph of $3 \%$ clay APB-BPDA films showing some collapsed clay 80

8. Reduced magnification micrograph of $3 \%$ clay APB-BPDA films 80

9. XRD patterns of air-cured APB-BPDA thin film samples 81

10. XRD patterns of air cured ODA-BTDA thin film samples 82

11. XRD patterns of sodium montmorrillonite and organoclay (SCPX-2003) 82

12. XRD patterns of $3 \%$ clay APB-BPDA poly(amide acid) hybrid mixture and film after air drying at room temperature

13. XRD patterns of $3 \%$ clay APB-BPDA films treated at different temperatures in air

14. XRD patterns of organoclay treated at different temperatures in air 
15. XRD patterns of $3 \%$ clay APB-BPDA films treated at different temperatures in nitrogen

16. XRD patterns of organoclay treated at different temperatures in nitrogen

17. Tensile modulus versus clay loading of air cured polyimide/organoclay systems

18. Elongation at break versus clay loading of air cured polyimide/organoclay systems

19. Yield Strength versus clay loading of air cured polyimide/organoclay systems

20. XRD pattern of DTMA organoclay

21. XRD pattern of DTMA/APB-BPDA nanocomposite film

22. XRD pattern of DTPP organoclay

23. XRD pattern of $B K 3-166 \mathrm{~A}$ organoclay

24. XRD pattern of BK3-166A/APB-BPDA hybrid film

25. XRD pattern of $B K 3-166 \mathrm{~B}$ organoclay

26. XRD pattern of BK3-166B/APB-BPDA hybrid

27. XRD pattern of BK3-166C organoclay

28. XRD pattern of BK3-166C/APB-BPDA hybrids

\section{Chapter 4}

1. Structure of cations used in organoclay preparation

2. Monomer structures

3. XRD patterns of fully charged organoclays

4. Diagram of cation orientation on clay surfaces

5. XRD pattern of reduced charge organoclays 
6. XRD pattern of fully charged organoclay prepared with an aliphatic amine

7. XRD pattern of RCM-2, 3, 6-TMPA ODA-BTDA hybrid films

8. XRD pattern of MCM-120-TMPA/ODA-BTDA hybrid film

9. XRD pattern of MCM-130-TMPA/ODA-BTDA hybrid film

10. XRD pattem of FCM-APB/APB-BPDA nanocomposites

11. XRD pattern of FCM-APB/APB-BPDA hybrid film

12. XRD pattern of RCM-250-APB/APB-BPDA polyimide

13. XRD pattern of FCM-TBPA/APB-BPDA hybrid films

14. XRD pattern of FCM-TMPA/APB-BPDA hybrid film

15. XRD pattern of FCM-TMPA/ODA-BTDA hybrid

16. XRD pattern of RCM-250-TMPA organoclay

17. XRD pattern of RCM-250-TMPA/APB-BPDA hybrid film

18. XRD pattern of RCM-250-TMPA/ODA-BTDA hybrid

19. XRD pattern of FCM-BP APB-BPDA hybrid film

20. TEM micrograph of FCM-BP/APB-BPDA hybrid film

21. XRD pattern of FCM-BP/ODA-BTDA film

22. XRD pattern of RCM-250-BP organoclay

23. XRD pattern of RCM-250-BP/APB-BPDA hybrid film

24. TEM micrograph of RCM-250-BP/APB-BPDA hybrid film 153

25. XRD pattern of RCM-250-BP/ODA-BTDA hybrid film

26. XRD pattern of FCM-TPP/APB-BPDA hybrid film

27. TEM micrograph of FCM-TPP/APB-BPDA hybrid film

28. XRD pattern of FCM-TPP/ODA-BTDA nanocomposite 
29. XRD pattern of RCM-250-TPP organoclay

30. XRD pattern of RCM-250-TPP/APB-BPDA hybrids

31. TEM micrograph of RCM-250-TPP/APB-BPDA hybrid film

32. XRD pattern of RCM-TPP/ODA-BTDA hybrids

33. XRD analysis of FCM-Na clay sample

34. XRD analysis of FCM-Na/ODA-BTDA hybrids

35. XRD pattern of RCM-250-Na+ clay 163

36. XRD pattern of RCM-250-Na+ ODA-BTDA hybrid film 164

37. Strength of APB-BPDA hybrid films 165

38. Modulus of APB-BPDA hybrid films 166

39. Strength of ODA-BTDA hybrid films 167

40. Modulus of ODA-BTDA hybrid films 168

41. TGA analysis of organoclays

Chapter 5

1. Structure of surfactant ions

2. Structure of monomers

3. XRD pattern of sodium montmorillonite

4. $\mathrm{XRD}$ pattern of $\mathrm{MCM}-\mathrm{Na}+$ clays

5. XRD pattern of APB organoclays

6. XRD pattern of MCM-130-APB organoclay

7. XRD pattern of BAOD organoclays

8. XRD pattern of MCM-APB/APB-BPDA hybrid films

9. XRD pattern of MCM-APB/APB-BPDA hybrid films 
10. XRD pattern of MCM-BAOD/APB-BPDA hybrid films

11. XRD pattern of MCM-BAOD/APB-BPDA hybrid films

12. XRD pattern of MCM-130-APB/APB-BPDA hybrid films

13. XRD pattern of MCM-130-BAOD/APB-BPDA hybrid films

14. Modulus of APB organoclay/APB-BPDA hybrid films

15. Elongation of APB organoclay/APB-BPDA hybrid films

16. Strength of APB organoclay/APB-BPDA hybrid films

17. Modulus of BAOD organoclay/APB-BPDA hybrid films

18. Elongation of BAOD organoclay/APB-BPDA hybrid films

19. Strength of BAOD organoclay/APB-BPDA hybrid films

20. Modulus of MCM-130-APB/APB-BPDA hybrid films

21. Strength of MCM-130-APB/APB-BPDA hybrid films

22. Elongation of MCM-130-APB/APB-BPDA hybrid films

23. Modulus of MCM-130-BAOD/APB-BPDA hybrid films

24. Strength of MCM-130-BAOD/APB-BPDA hybrid films

25. Elongation of MCM-130-BAOD/APB-BPDA hybrid films

26. TEM micrographs of 3\% MCM-130-APB/APB-BPDA nanocomposite film

27. TEM micrographs of 5\% MCM-130-APB APB-BPDA nanocomposite film

28. TEM micrographs of $8 \%$ MCM-130-APB/APB-BPDA nanocomposite film

29. TEM micrographs of 3\% MCM-130-BAOD/APB-BPDA nanocomposite film

30. TEM micrographs of 5\% MCM-130-BAOD/APB-BPDA nanocomposite film

31. TEM micrographs of $8 \%$ MCM-130-BAOD/APB-BPDA nanocomposite film 
33. TEM micrographs of 3\% MCM-170-BAOD/APB-BPDA nanocomposite film 224

34. TEM micrographs of 3\% FCM-APB/APB-BPDA nanocomposite film 226

35. TEM micrographs of 3\% FCM-BAOD/APB-BPDA nanocomposite film 227

36. TEM micrographs of 3\% MCM-120-APB/APB-BPDA nanocomposite film 229

37. TEM micrographs of 3\% MCM-120-BAOD/APB-BPDA nanocomposite film 231

38. TEM micrographs of 3\% MCM-140-APB/APB-BPDA nanocomposite film 232

39. TEM micrographs of 3\% MCM-140-BAOD/APB-BPDA hybrid film 233 


\begin{abstract}
The purpose of this research was to prepare nanocomposite materials comprised of exfoliated clay particles in a polyimide matrix. Poly(amide acid)/organoclay solutions and polyimide/organoclay films were prepared and the clay dispersion was characterized by visual inspection, XRD, and TEM. Mechanical measurements and certain thermal characterization measurements were also obtained. The research began by attempting to repeat the procedures set forth in the literature. Most of the polyimide/organoclay nanocomposites were being prepared by mixing prepared poly(amide acid) solutions with organoclay solutions. This simple mixing technology was used in the preparation of various polyimide/organoclay hybrid formulations. In-situ polymerization, which involved performing the polymer synthesis in the presence of the organoclay, replaced simple mixing with increased success. Although the in-situ polymerization technique was successful at exfoliating clay particles in certain polyimides, the organoclay degraded at the polyimide cure temperature. In order to raise the use temperature, the aliphatic surfactants found in commonly used organoclays were replaced with aromatic surfactants. The dispersion of the clay was more difficult with the aromatic surfactants. It was facilitated by reducing the charge on the clay surface. This was aceived by replacing some of the cations that reside on the surface of the clay particles with lithium ions in the interior of the particles. The in-situ polymerization of APB-BPDA poly(amide acid) in the presence of an aromatic organoclay with a cation exchange capacity (CEC) of $0.57 \mathrm{meq} / \mathrm{g}$ and subsequent cure to the polyimide yielded a film with a high level of clay dispersion.
\end{abstract}




\section{PREPARATION AND CHARACTERIZATION OF POLYIMIDE/ORGANOCLAY NANOCOMPOSITES}




\section{Chapter I}

\section{Introduction}

The field of polymer science has been changed with a single word: NANO. There is not an engineer in the world that has not at least heard the phrase nano-materials in some context. The tidal wave of research in this area began in the late 80 's and has gained tremendous height and breadth as it has traveled through the scientific community. It is safe to say that the wave has not crashed yet and by all rights should not be considered as being close to its cresting height. There is an entire scientific community devoted to technology in which particles of nanometer dimensions are being used to enhance an already expansive field of polymer technology. Nanocomposites have and will become the future of all polymer science as the technology for preparation of these materials meets the demands for the material.

Polymeric materials have been used in industrial applications since the 1930 's $\mathrm{s}^{1}$. The use of these materials has allowed other technologies to further develop, as the materials needed for various applications were available. The technology increase since the 1950's was no less than astounding and the increase in technology has never been at a faster pace at any other time in history. This explosion was partly fueled by new materials that could support the ideas of the inventors, but quickly the creativity of inventors surpassed the resources available in the materials world. The challenge that materials scientists are faced with today are exciting and pushes the technology in this area to constantly look for novel materials.

Material scientists and more precisely polymer scientists have always been in search of new materials for use in replacement materials and for use in new applications. The 
obtained with conventional fillers is 0.1 to 10 microns $s^{1}$. Hybrid materials prepared with particles in this size range have properties that follow the simple rule of mixtures. Hybrid polymer/filler materials exist in many different combinations of polymers and fillers. Filled polymeric materials have been studied quite extensively.

It is clear from research involving filler particles in polymer matrices that smaller particle sizes allow for greater enhancements in some of the properties. The concentration of interphase greatly increases when the particle size is reduced. In fact when particles reach nanometer dimensions almost the entire material is considered interphase $^{2}$. For these reasons particles that will disperse to nanometer dimensions are very interesting to the scientific community. The fillers that were used in the early stages of filler technology had larger sizes than nanometer dimensions, but it was later discovered that certain metals and clays could be dispersed to nanometer dimensions. The clays are the focus of this research and have been successfully dispersed to nanometer dimensions in many polymeric matrices including the polyimides. In order to better understand these nanocomposite materials it is important to discuss the building blocks and the general technology of hybrid formation and characterization.

The polyimides represent an important class of polymers within the family of polymeric materials. These condensation polymers are known for their high strength and high temperature properties. These interesting properties make them highly desirable for space, aeronautic, and electronic applications and many other applications. Although polyimides have many potential uses they are relatively high cost materials which limits their widespread use. The special demands found in space applications have made polyimides a high priority for study at NASA-Langley Research Center (NASA-LaRC). 
The polyimides have been thoroughly studied both at NASA and other research facilities. Many advances in monomer synthesis, polymer preparation, processing, and characterization have been made in recent years. In efforts to create even higher performance materials, the polyimide materials were chosen as the host polymer in clay nanocomposite preparation. The polyimides were of special importance to NASA and the project described herein focused on placing nanoclays into polyimide materials.

Polyimides are usually prepared in two steps ${ }^{3}$. The first step is the preparation of the poly(amide acid) (PAA) followed by a second step where the amide acid is converted to an imide. The PAAs are prepared in a condensation reaction involving a diamine and a dianhydride. This reaction is usually performed in a polar aprotic organic solvent such as $\mathrm{N}$-methyl-2-pyrrolidinone (NMP). The diamine is usually added to the solvent and dissolved prior to the addition of dianhydride. The diamine is usually added as a dry powder while the dianhydride can be added as a slurry or a dry powder. By adjusting the stoichiometric quantities of the monomers, the molecular weight of the resulting polyimide can be controlled. A number of methods can be used to convert the PAA to the polyimide. Thermal treatment of the poly(amide acid) is the most common way to perform this cyclodehydration to effect imidization. The thermal treatment of the poly(amide acid) is usually performed at temperatures above $250^{\circ} \mathrm{C}$ and sometimes is performed at a final temperature of $300^{\circ} \mathrm{C}$. Another method used to for cyclodehydrate the PAA involves removing the water as it forms an azeotrope with toluene at temperatures below $250^{\circ} \mathrm{C}$ and then removing the toluene/water complex from the reaction. A third method to perform the ring closure is to chemically imidize the chain by placing an excess of both pyridine and acetic anhydride in the reaction vessel with the 
PAA. This will effectively close the ring and the polyimide. The polyimide is usually insoluble in polar aprotic solvents and will precipitate. In all three methods the relatively chemically inert imide ring is formed.<smiles></smiles>

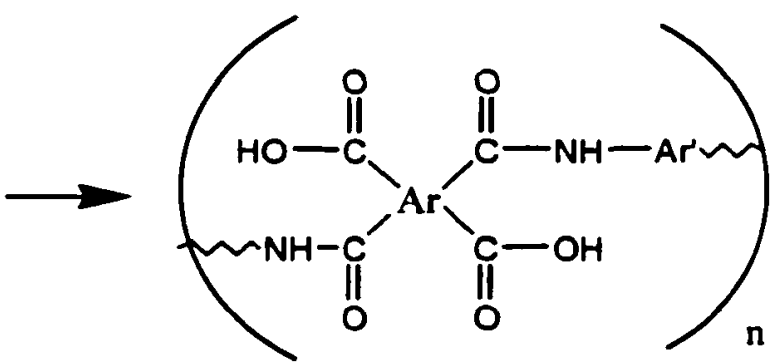

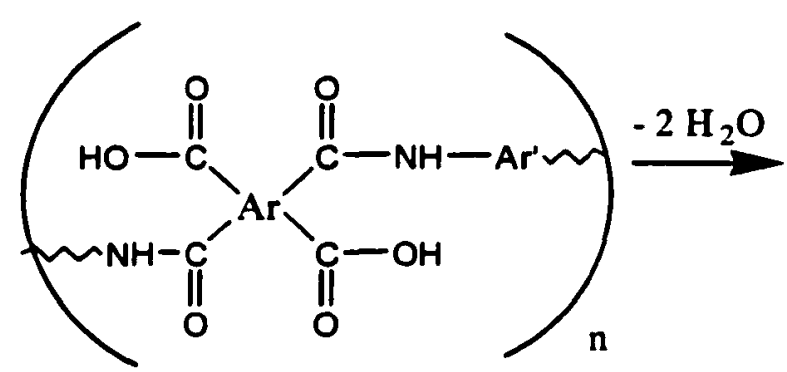

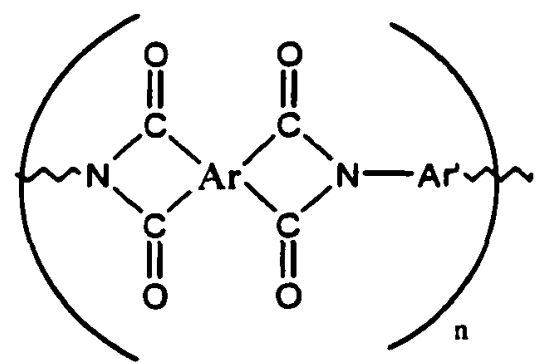<smiles>O=C1OC(=O)c2ccccc21</smiles>

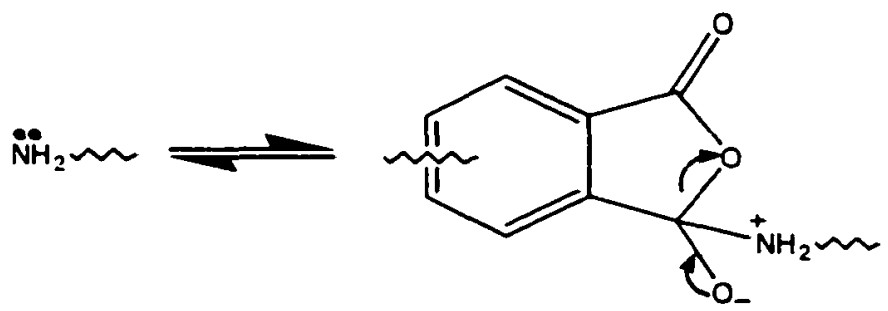<smiles></smiles><smiles>C=CC=C</smiles><smiles>CNC(=O)c1ccccc1C(=O)O</smiles>

Figure 1. Mechanism of PAA formation ${ }^{3}$ 
The clay minerals are naturally occurring particles that are mined and refined to a lab grade product. The clays that are most commonly used in the preparation of nanocomposites are montmorillonites and hectorites. These clays are expandable clays, which means that the individual clay particles can be separated but remain in bundles of many clay particles ${ }^{4}$. The structure of an individual clay particle is quite complex and is made of two layers of silicon dioxide that sandwich a layer of metal atoms. The silicon dioxide and the metal layer share oxygen atoms at their interface. The silicon atoms are covalently bonded to the shared oxygen in a tetrahedral arrangement while the metal atoms are covalently bonded to the oxygen atoms in an octahedral arrangement. The metal atoms are usually aluminum in the montmorillonites and magnesium in the hectorites. These metal atoms are sometimes substituted in nature by lower valence ions resulting in a charge imbalance. This charge imbalance is manifest at the surface of the clay particles and is usually compensated by a counter-ion such as sodium. Figure 2 is a representative structure of montmorillonite clay. 

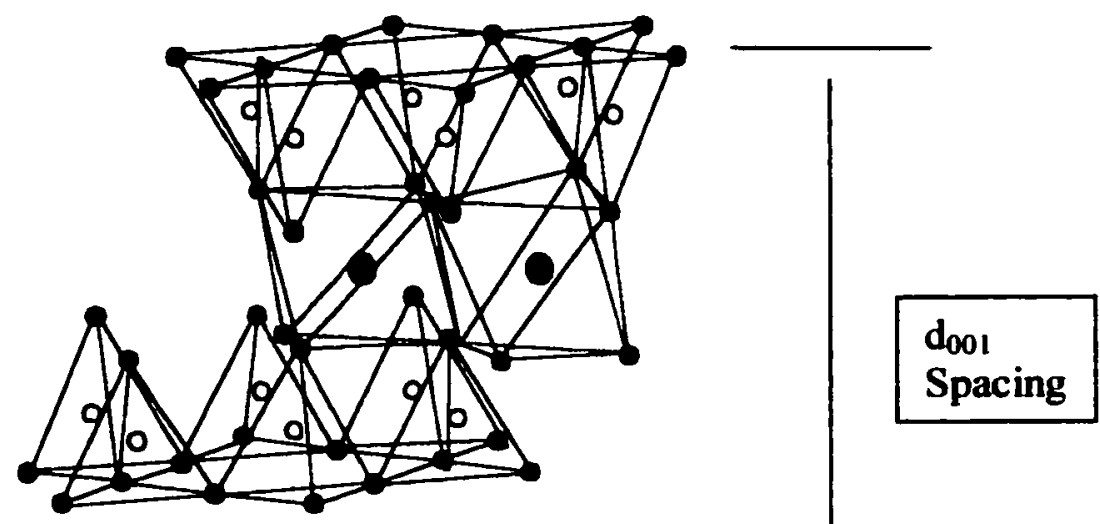

Blue-Metal (Al or Mg)

Red- Oxygen

Yellow- Silicon

Green- Hydroxyl

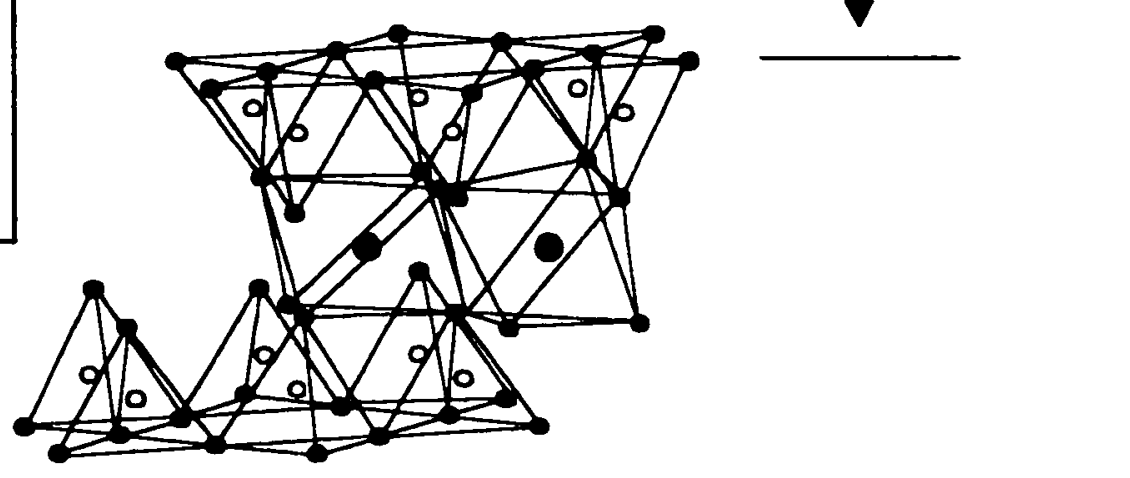

Figure 2. 3-D representation of montmorillonite clay ${ }^{4}$

The space between the clay platelets is considered the inner layer region or gallery. This region can be expanded or contracted and the size of this region is usually related to the size of the ion in the gallery. The $d_{001}$ spacing or the $d$-spacing is commonly reported for an organoclay and includes the inner layer space and the thickness of one of the clay particles. This $\mathrm{d}$-spacing is the distance between two identical faces in the stack of clay particles. This $\mathrm{d}$-spacing is measured by $\mathrm{X}$-ray diffracion $(\mathrm{XRD})$ and can be calculated using equation 1.

$$
\mathrm{n} \lambda=2 \mathrm{~d}_{00 !} \sin (\theta)
$$

The wavelength $(\lambda)$ is the wavelength of the source radiation and is usually the wavelength of $\mathrm{Cu} \mathrm{K} \mathrm{K}_{\alpha}$ radiation. Theta $(\theta)$ is the angle of the incident radiation relative to 
the sample. The value for $n$ is usually one. The $d$-spacing can be measured for the neat clay and clay in polymer matrices provided that some degree of order exists. The clay particles themselves and the inner layer space are important features of the clay, but a complete description of the clay is needed to better understand the polymer clay nanocomposite materials.

The clay minerals are interesting in many aspects including their distinctive morphology and their behavior in certain liquids. Montmorrillonites have a hexagonal plate structure with single plate dimensions of $1-10 \mathrm{~nm}$ thick and $100-200 \mathrm{~nm}$ on each side $^{5}$. Of course the particles do not exist naturally as separate entities but rather come in stacks of many clay sheets. The sheets are much longer than $200-300 \mathrm{~nm}$ on each side as found in the platelets. The sheets exist in stacks as well. The ionic forces on the clay surface are compensated with sodium atoms on the surface. Two clay particles sandwich a layer of sodium atoms and stack of clay particles results. The particles behave almost like ionic salts when placed in water ${ }^{6}$. At low concentrations $(<1 \%)$ the clay in water can almost be considered a solution. The particles will separate into individual clay platelets and disperse into what is known as a sol. The particles in these clay/water mixtures scatter light, which in turn gives the solution a distinctive color depending on the color of the original clay ${ }^{3}$. The ability of the clays to disperse in aqueous media like this is an important feature because it allows the surface ions to be exchanged with other watersoluble salts.

The naturally occurring clay minerals are usually not used in nanocomposite preparation without a surface modification. Preparing a colloidal suspension or sol of the clay in water followed by the addition of excess of another salt results in easily 
exchanging the original ion. The salt that is most abundant in the solution will dominate the surface of the clay forcing the original salt to leave. The ion exchange process is depicted below in Figure 3, showing the replacement of sodium ions by a nitrogencontaining organic cation.
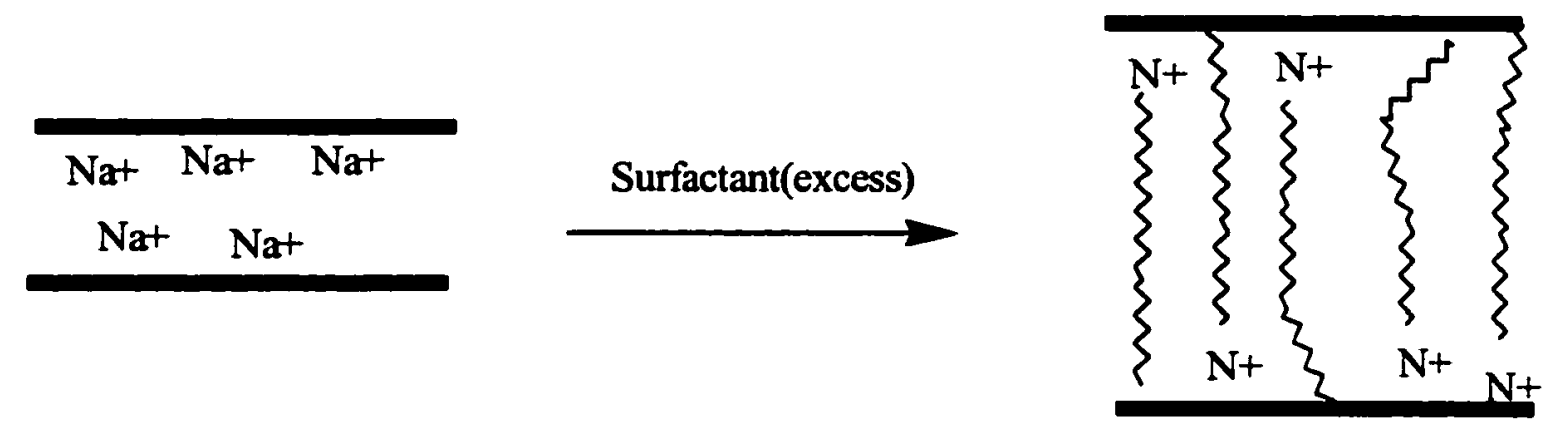

Figure 3. Diagram of ion exchange process

Organic salts are commonly used to prepare the organoclays that are used in nanocomposite preparation. For the applications in this study the organic salt molecules have the properties of a surfactant. The positively charged atom of the surfactant attaches to the surface while the other part of the molecule, which is usually an aliphatic chain, moves away from the surface. Surfactant ions are usually tailored to maximize the positive interactions of the surfactant and the polymer molecule. Surface modification and surfactant chemistry are widely studied fields and will be discussed in great detail throughout the text. The organically modified clays are an integral part of nanocomposite preparation.

The polyimides and the organoclays are the building blocks for polyimide/organoclay nanocomposite materials and are presented in the above text. As separate entities these building blocks are well defined. The challenge in preparing high quality nanocomposite materials is to effectively disperse the clay from their naturally 
occurring bundles into individual clay platelets. When polymers and organoclays are mixed there are three different morphologies that can dominate the system. The polymer and the clay can be totally immiscible (i.e. in isolated clay bundles in the polymer matrix). Secondly, the polymer/organoclay blend is said to be "intercalated" when one or two polymer chains enter the clay inner layer but the particles are still ordered. Thirdly the system can have clay that is exfoliated and completely disordered. This is usually the desired form for the polymer nanocomposite. The different morphologies are shown in figure 4.

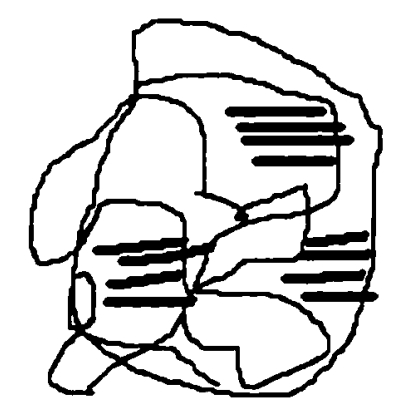

Immiscible

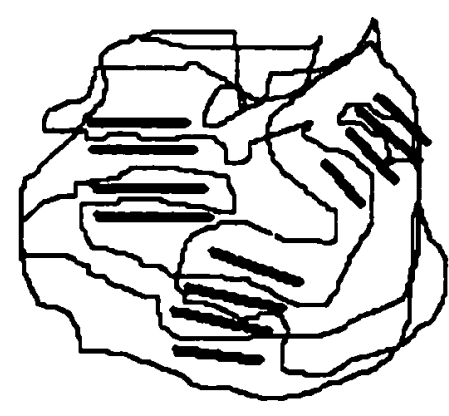

Intercalated

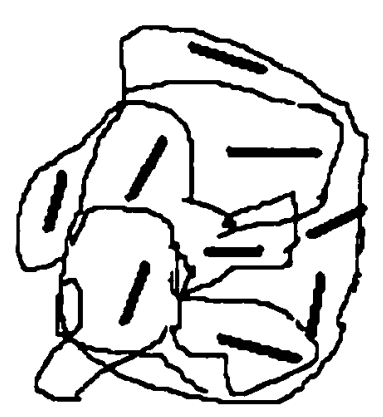

Exfoliated

Figure 4. Possible hybrid morphologies ${ }^{5}$

The clay/polymer products shown in figure 3 are idealized scenarios but it is also possible to have combinations of these three morphologies. For example, a polymer/organoclay blend may have regions where the clay is exfoliated and other regions where the clay is intercalated. A system can also exist that has the same morphology throughout the system but the morphology is between the ones described above. For example, a system can have exfoliated clay particles in which the clay particles have been separated by more than $-3.5 \mathrm{~nm}$ but the there can still be some order in the polymer matrix. Although not entirely descriptive, the three terms immiscible, intercalated, and exfoliated are used most 
commonly used to describe clay/polymer systems and will be used throughout the text.

The properties of the systems differ greatly one from another. Generally, the desired properties enhancements are of course expected when the particles are exfoliated and are of nanoscopic dimensions.

The goal in polymer/organoclay hybrid formation is to control the dispersion of the clay and in most cases is to exfoliate the clay. There are many factors to consider when preparing nanocomposite materials. These restrictions stem from the preparatory techniques of the polymer and the low probability of expanding the clay layers in organic solvent. The research presented herein discusses preparation techniques as well as different organoclays that can be used to prepare nanocomposite materials. The preparatory techniques used in preparing nanocomposites are numerous and vary for different polymer systems. One of the most common ways that nanocomposite materials are prepared is to simply mix the clay with the polymer. This can be done as the polymer is molten or in a solvent that is common to both clay and polymer. The simple mixing technique was used with polyimides ${ }^{7}$, polyether $^{8}$, polystyrene $^{9}$, epoxies ${ }^{10}$, poly(methyl methacrylate $)^{11}$, polysulfone ${ }^{12}$, as well as many other polymers. Special equipment, such as extruders, sonicators, and melt mixers, is often used to aid the simple mixing process. The use of all these mechanical mixing methods are considered simple mixing throughout this paper. Simple mixing is defined as any mechanical means of blending a polymer with an organoclay. Another technique is presented in which monomers are added to the clay/solvent dispersion or the polymerization is carried out by some other means in the presence of the clay. This method is considered in-situ polymerization. The in-situ polymerization technique is used in a variety of polymer systems as well including 
polyimides ${ }^{13}, \mathrm{PMMA}^{14}$, polyurethane ${ }^{15}$, butadienes-acrylonitrile copolymer ${ }^{16}$, and others that can be prepared this way. The in-situ polymerization technique has proven to be successful in many instances and examples of its uses are becoming more abundant. Different combinations of mixing techniques can also be used in special cases to disperse the clay particles. The mixing scheme is important in preparing nanocomposite materials and will be discussed throughout this paper.

Characterization is necessary to determine the effect different components such as preparation method, polymer, or surfactant have on the entire system. The characterization methods for determining whether or not quality nanocomposites have been formed are somewhat limited. There are a variety of techniques used to determine the clay dispersion in nanocomposite materials including visual inspection, $\mathbf{x}$-ray diffraction (XRD), transmission electron microscopy (TEM), atomic force microscopy (AFM), and nuclear magnetic resonance (NMR). Although these techniques can often determine whether or not an organoclay is dispersed to individual clay platelets, it is difficult to determine relative levels of clay dispersion. The different factors involved in preparing nanocomposites make it very helpful that relative levels of dispersion be assigned. Without the ability to determine levels of dispersion it becomes almost impossible to determine the effects of changing one of the factors. This is not only true when comparing a specific preparations but becomes important when comparing materials prepared in the lab with those presented in the literature. The lack of a good system for assigning levels of dispersion in clay nanocomposites has been a major detriment to progress in this field. The building blocks are clearly defined but the hybrid materials are difficult to characterize. 
An example of the difficulty in characterizing the dispersion in these nanocomposites can be found in the TEM micrographs of a hybrid material taken at different resolutions or different magnifications. The micrograph may also show only a portion of the material where the clay is better dispersed than the rest of the film, which would make the results somewhat misleading. For these reasons the use of TEM as the only characterization instrument in determining the dispersion of clay is not sufficient. A comparison of a number of TEM micrographs from the literature and their accompanying text are presented below to further illustrate the problem with this type of characterization.

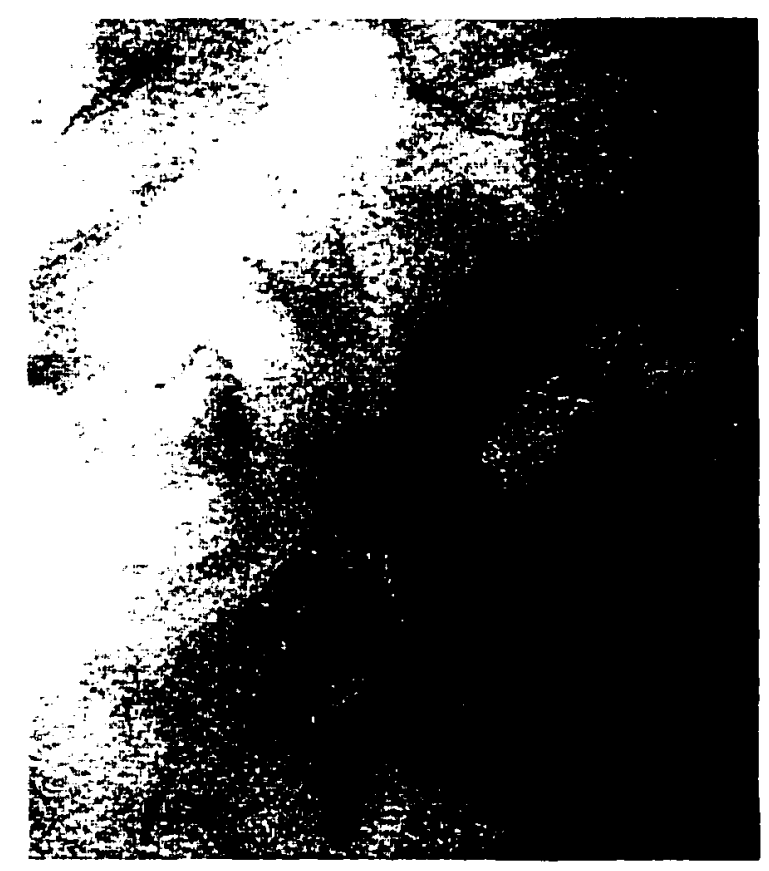

Figure 5. TEM of a disordered PMMA nanocomposite material ${ }^{2}$

The picture shown here has no scalebar; but based on the size of the particles, it appears that the magnification is above 20000x. The particles are well dispersed in this micrograph but there is some agglomeration. The author claims that this material is exfoliated ${ }^{2}$. As discussed earlier, the terminology (i.e., exfoliated, intercalated, and immiscible) used to describe nanocomposite preparations is too broad and needs to be 
more clearly defined. For a material to be considered truly exfoliated then all the particles faces need to be separated to a distance that is more than $\sim 3.5 \mathrm{~nm}$ away from every other particle face. Again this may be the case in some areas of a material but if the entire material is not that way then the material should not be considered exfoliated. A TEM micrograph (figure 6) of a polysulfone organoclay nanocomposite further illustrates this point.
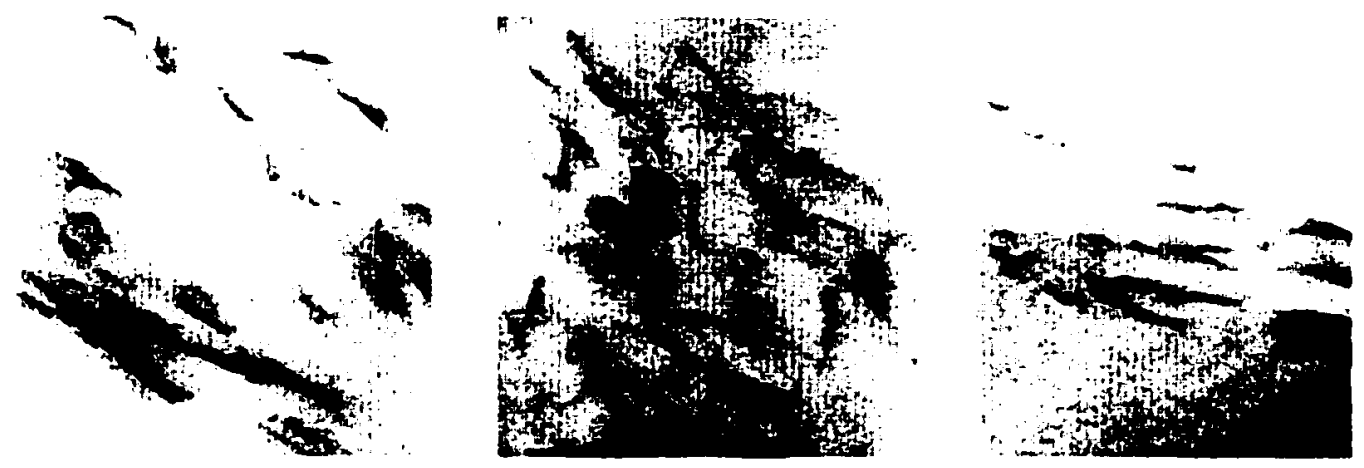

Figure 6. TEM of a polysulfone/organoclay nanocomposite This figure is also representative of what the author considers exfoliation ${ }^{16}$. There appears to be some agglomeration in this material and looks as if the clay is not exfoliated. There may be some exfoliation in this material but it is limited.

A third illustration of the problems associated with charcterization of these films another TEM micrograph is shown below in figure 7. 


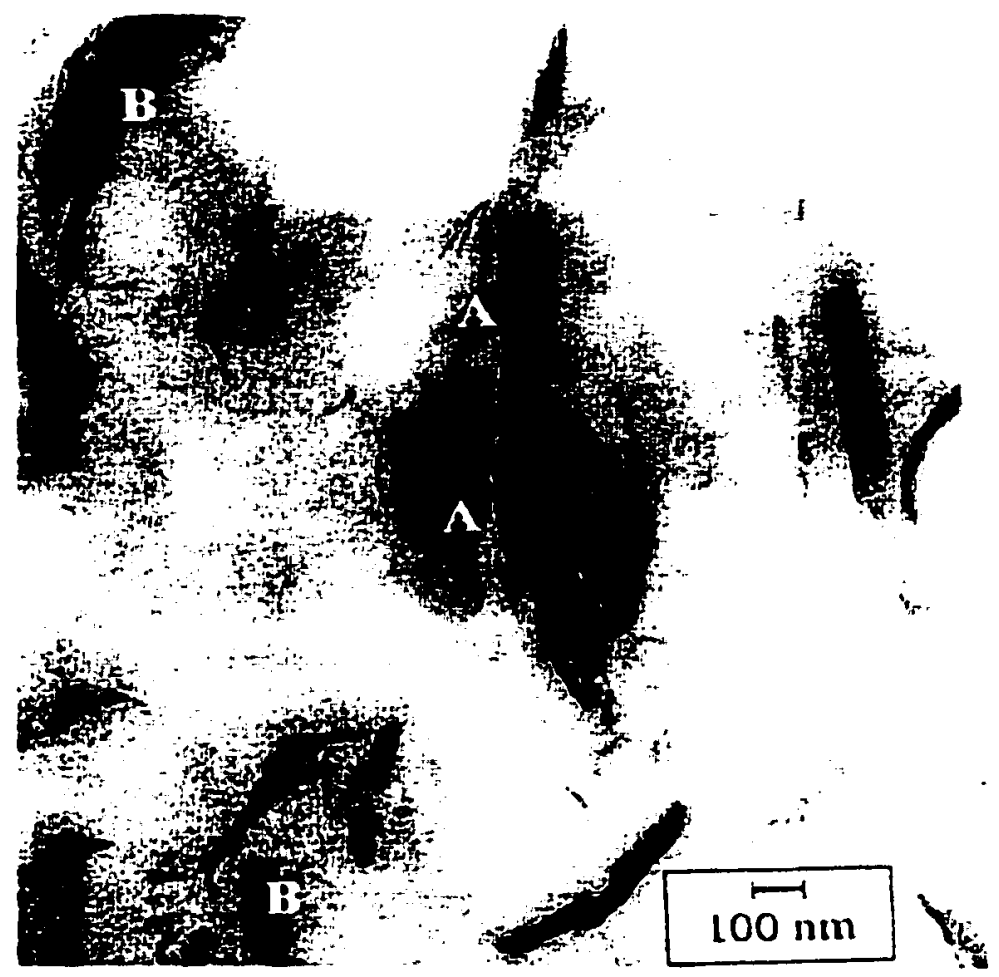

Figure 7. TEM micrograph of a polypropylene/montmorillonite nanocomposite material This is a TEM micrograph of a polypropylene nanocomposite material and the regions labeled $B$ are considered to be exfoliated by the author while the particles labeled A are considered intercalated $^{15}$. This identification of both dispersion characteristics in the same film is interesting although the areas that are labeled as exfoliated appear to be agglomerated as well but the particles are just a slightly smailer size. Further illustration at higher concentrations is presented below in figure 8 . 


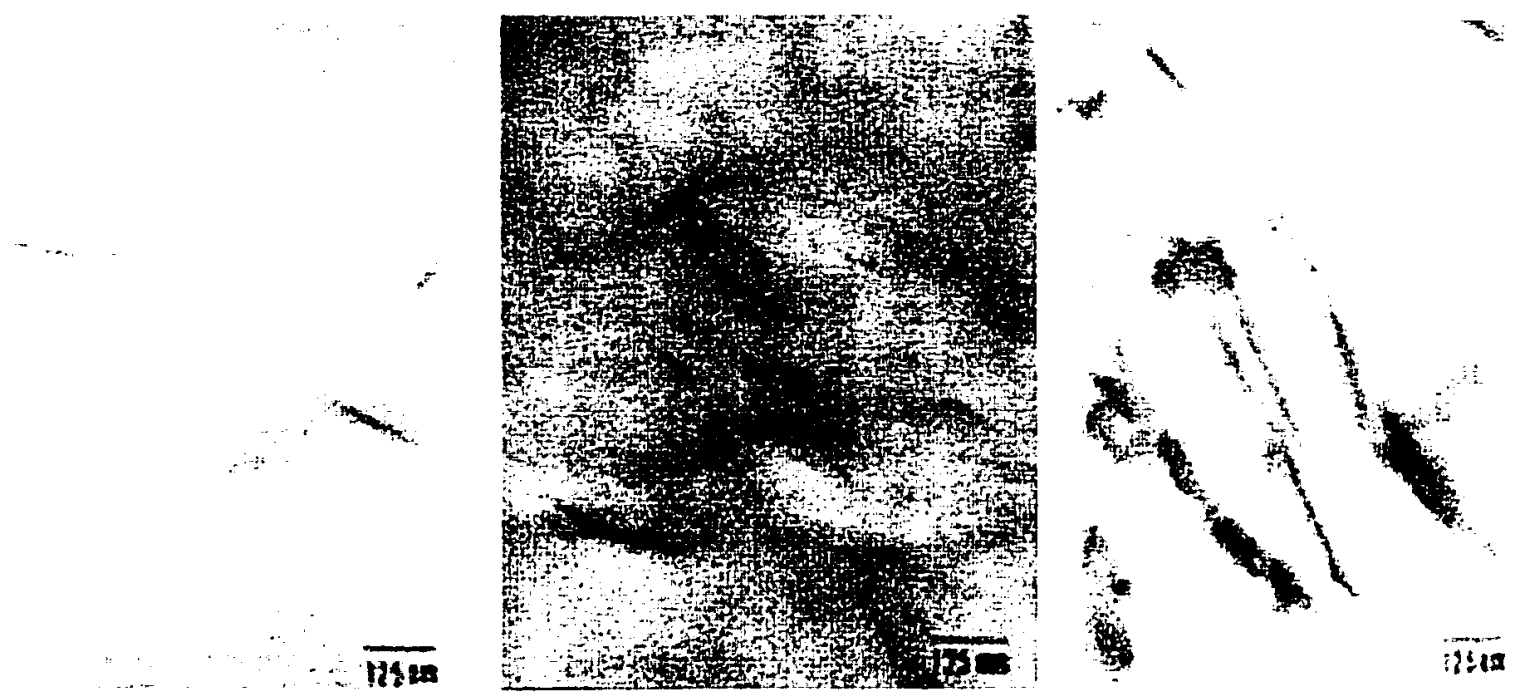

Figure 8. TEM micrographs of polysulfone/MMT nanocomposites at 1,3 , and $5 \%{ }^{10}$

These micrographs are from left to right represent 1,3 , and $5 \%$ clay loaded polysulfone. The author states the clay starts out exfoliated in the first micrograph and becomes more agglomerated as the concentration increases. This looks to be the case and the amount of clay in each frame seems to follow the trend with concentration as the higher concentration films have more particles in them. It is hard to see what the entire film looks like in this example. There are a few particles in the frame at this magnification but it would be interesting to see what the overall dispersion was. This cannot be determined from these micrographs.

The next figure (figure 9) is a TEM micrograph of exfoliated montmorillonite in polystyrene ${ }^{7}$. The particles in this film do look as if they are well dispersed but again this is taken at extremely high magnification and it is hard to tell if this is representative of the entire material. 

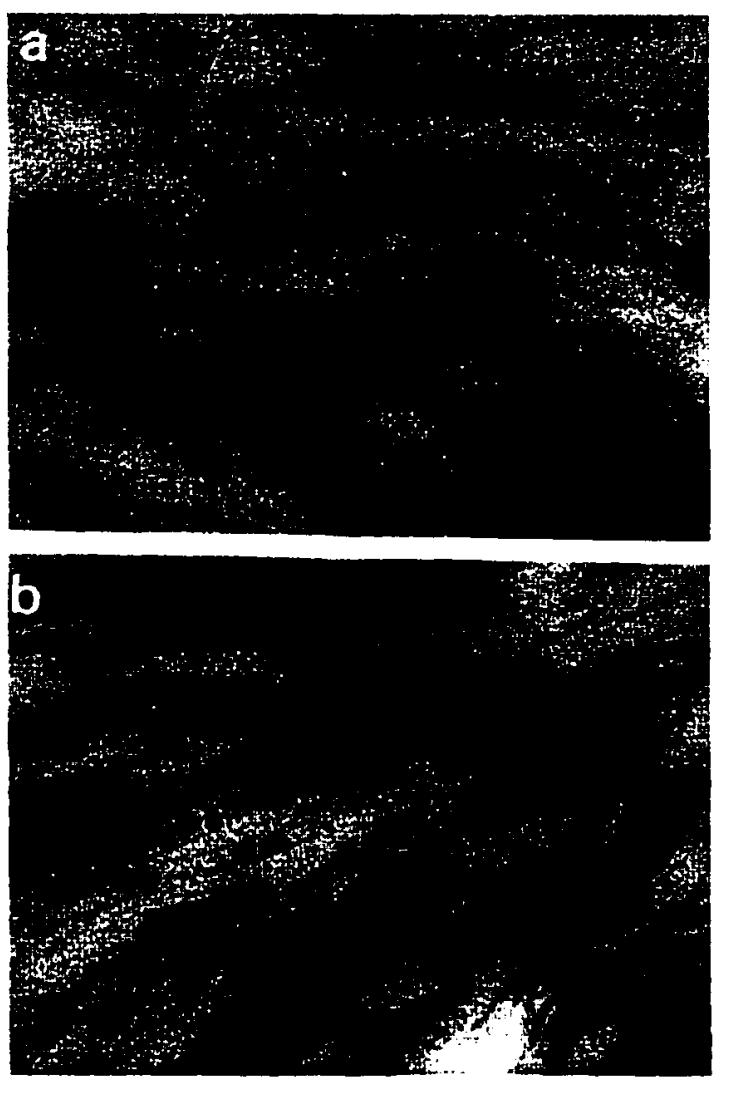

$100 \mathrm{~nm}$

Figure 9. TEM of polystyrene/montmorillonite nanocomposite ${ }^{7}$

The results shown here do illustrate the difficulty with discussing dispersion of clay particles in nanocomposite materials. The designation of exfoliation was given to a number of samples here and yet the particles all had different morphologies. The data obtained from TEM micrographs are difficult to communicate and makes it difficult to determine the levels of clay dispersion relative to the samples in the literature.

Characterization using a single investigative tool such as TEM is ineffective for determining the level of dispersion of a single hybrid material and has proven to be a less than perfect method for comparing different hybrid preparations. For this reason the TEM micrographs are usually accompanied by other characterization such as XRD. The $\mathrm{XRD}$ pattern of a material yields valuable information about the spacing of clay particles 
to one another. A peak in the XRD pattern obtained of a specific material indicates that clay particles in that sample are ordered. The angle at which the peak occurs indicates the spacing of the clay particles. The XRD patterns are useful for characterizing a hybrid material but again the data from this investigative tool can be quite misleading if let stand alone. The XRD pattern cannot be used as a quantitative measurement in the analysis of hybrid materials and therefore cannot be used in determining the level of dispersion from sample to sample. The intensity of an XRD pattern is indicative of the amount of clay in the beam with the indicated d-spacing. The problem lies in the samples themselves and the different combinations of dispersed clay particles that would yield the same XRD pattern. This effect is illustrated below in figure 10.
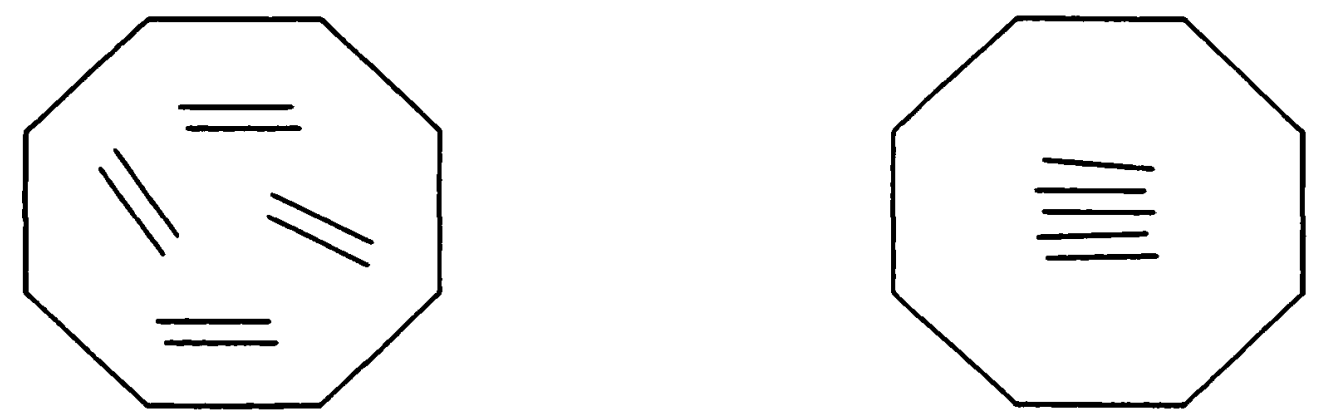

Figure 10. Representative nanocomposite samples for XRD analysis

This figure is used to represent two identical areas of a nanocomposite material. The lines in the middle of the octagon represent clay particles and are representative of two different clay dispersion patterns. Although the dispersion patterns are quite different the XRD pattern for these two samples would be identical assuming that the clay particles $d-$ spacing's are identical. The scattering angle would be the same and the intensity of the peak would be indicative of four particles having this spacing. This illustrates the need for care when interpreting XRD patterns for the level of dispersion in a given material. There are also problems with nanocomposite materials in which the clay is so 
agglomerated that there are large areas of the material void of clay. If this is the case it is possible to get an XRD pattern without a peak indicating exfoliation by scanning an area that has no clay. These problems have hindered the use of XRD in assigning levels of dispersion.

In an attempt to determine which nanocomposite preparations are most effective at dispersing the clay preparations it is desirable to quantify dispersion. Yet, as is stressed here, this is difficult. XRD and TEM are good investigative tools for nanocomposite characterization but are not sufficient by themselves or together to determine the level of dispersion. The visual appearance of the hybrid materials can yield a good estimation of how well the particles are dispersed and can be used in conjunction with the other two techniques. The visual appearance of the film covers a much larger area than the other two investigative techniques and can add to the validity of the data from the TEM and XRD or can completely nullify the results from these two techniques. For this reason the visual inspection of the material is an integral part of the characterization process and must be included in the discussion of the overall characteristics of the material.

The homogenous dispersion of clay particles in a polymer matrix is the goal of nanocomposite research and the level to which this is accomplished is studied by the different characterization techniques discussed above. Another objective for this research was to enhance the properties of the neat polymer materials including the mechanical properties. The mechanical properties or the bulk properties of a material can also be used to discuss dispersion characteristics to some degree. There should be a direct relationship between mechanical properties and the particle sizes of the filler materials. 
The stiffness of a filler-containing material is considered to be dependent on the size of the filler that is used ${ }^{17}$. Smaller particles usually enhance the stiffness or modulus of a material to a greater degree than larger particles of the same total mass. The increased stiffness is attributed to an immobilization of the polymer shell around the filler. This immobilized layer is more widespread throughout the sample as the particle size is reduced. Increasing the aspect ratios and filler concentration also play a role in the stiffness of a material. The stiffness of a material should increase as the aspect ratio and the filler concentration increase. Fillers that have plate or fiber morphology have high aspect ratios. The clay particles exist as plates when they are exfoliated. The clay particles have a high aspect ratio and the modulus is usually greatly enhanced ${ }^{17}$. The use of nanoclays was very attractive for modulus enhancement in the polyimide materials. The toughness or the impact strength of a material is another property of polymeric material that is affected by filler addition. Although polymer immobilazation increases the stiffness it also decreases the toughness of a material. For this reason a high concentration of small particles is predicted to be detrimental to the toughness of a material. Toughness is also reduced by adding a small amount of particles which are larger than $\sim 10$ microns. The relationship between toughness and particle size is not completely understood ${ }^{17}$. In general, the elongation properties follow the same trend as toughness.

The tensile strength of materials is also affected by the addition of particles to the matrix. The reported tensile strengths of nanocomposite materials fluctuate from system to system. It is unclear whether the measurement technique is not accurate in some cases or if slight modifications in preparation change the strengths of these materials drastically. 
It is known that the large agglomerate can adversely affect the tensile strengths of a material in the system. Other factors are of course involved in determining the strength of a material, but they will not be presented here. There is not a general rule to follow when comparing tensile strengths of clay filled materials.

The mechanical properties that are reported for the systems discussed in this work include Young's modulus, tensile strength, and \% elongation. Comparisons are made with the properties of similar systems in the absence of nanoparticles. The general predictions assigned to these properties when filler and especially nanoclays are added are discussed above. The mechanical properties were measured using an instron tensile testing machine. The average value of five specimens for each sample is reported. Although the mean is reported the result for each specimen is not listed. Reporting the value obtained for each individual specimen would be very time consuming and is not necessary. In all the samples, the averages were compared in hopes of exposing a trend in the data, but even if no outstanding differences were witnessed then the averages were compared. There are reasons why the mechanical data are not examined more closely. First of all the hybrid solutions used in this work were prepared using many different procedures. Although the molecular weight of the poly(amide acid) or the polyimide was kept as uniform as possible the actual molecular weight could not be determined in most cases. Extreme caution was taken to keep the films as clean as possible but the thin films were prepared without the use of a clean room and therefore were exposed to some foreign particles. The mechanical properties were meastired for these materials to determine if any major differences could be detected. The mechanical data were included 
in some cases even if it was not peculiar in some way. These data are generally discussed in order to reveal any trends.

The effect of filler particles on the color and transparency of materials is also important for a lot of applications. Impurities in the raw filler can drastically affect appearance. The consistency or the homogenous appearance of a hybrid material is closely linked to the homogeneity of the dispersion of filler. Color changes in a hybrid material are caused by a variety of factors including the size, color, and refractive index of the filler material. When particles reach nanoscopic dimensions they are not supposed to alter the optical clarity of the material because the particles are smaller than the wavelength of visible light. This is not always the case and other phenomona such as light scattering and refractive index inconsistencies sometimes occur. These optical effects are quite complex and are beyond the scope of this research. The appearance of the hybrid materials is discussed in the text.

The work presented herein was focused on developing a method to prepare welldispersed polyimide/organoclay nanocomposite by any means necessary. To accomplish this task a host of materials and blending techniques were employed. A wealth of knowledge was obtained on this class of materials and will be used to further develop nanocomposite technology. The research presented herein naturally divided into four sections. The first section involved all the materials that were prepared via a simple mixing process. This section included many different polymer/organoclay combinations. The simple mixing technique was not the best technique for blending polyimides with organoclays but some success was obtained when high shearing forces were imparted on the hybrid solutions with a homogenizer. The remaining three sections all used in-situ 
polymerization as the means of blending the polymer and organoclay. The in-situ polymerization technique was found to be a much better way of preparing polyimide nanocomposites. This technique was one in which the polymerization was performed in the presence of the organoclay. The second section of this dissertation focuses on using the in-situ polymerization approach with conventional organoclays. These organoclays were modified with surfactants that contain long aliphatic chains as one of the functional groups on the cation. Although these groups did facilitate clay dispersion in the hybrid solutions it was quickly determined that they were degrading during the cure cycle. The third and fourth section of this work focused on preparing organoclays that would disperse as well as the aliphatic based organoclays but that would not degrade at the polyimide cure temperature $\left(300^{\circ} \mathrm{C}\right)$. The third section was focused on developing aromatic organoclays that had these properties. It was determined rather quickly that a fundamental understanding of these organoclays was required and thus chapter three is devoted to studying the various parameters associated with this type of organoclay. After obtaining some important principles from the third section of research the fourth section was devoted to applying the principles to create the best organoclay for a particular polyimide. The fourth section of research yieided an organoclay that would withstand the high temperatures encountered in polyimide preparation and that dispersed as well if not better than the conventional organoclays discussed in chapter two. The work was concluded with this discovery and a limited discussion on the reasons why it worked. 


\section{Chapter I References}

1) Jancar, J. Advances in Polymer Science, Mineral Fillers in Thermoplastics 1, 139, 1999.

2) R. Krishnamoorti and R. Vaia. Polymer Nanocomposites, ACS Symposium Series No. 804, 2001.

3) Wilson, Stenzenberger, and Hergenrother, editors. Polyimides, 1990.

4) Grim. Clay Mineralogy, 1953.

5) R. Vaia, E. Gianellis. Macromolecules 1997; 30:7990.

6) Van Olphen , Ed., "An Introduction to Clay Colloid Chemistry, Second Edition", Krieger Publishing Company, Malabar, Florida, 1991.

7) H. Tyan, Y. Liu, and K. Wei, Polymer, 1999;40:4877-4886.

8) W. Zhou, J. Mark, M. Unroe, and F. Arnold. J. Macromolecular Sci.-Pure Appl. Chem. A38(1), 1-9(2001).

9) X Fu, and S. Qutubuddin. Polymer, 2001;42:807-813.

10) X. Kornman, H. Lindberg, and L. Berglund, Polymer, 2001;42:1303-1310.

11) M. Okamoto, S. Morita, Y. Kim, T. Kotaka, and H. Tateyama. Polymer, 2001;42:1201-1206.

12) G. Sur, H. Sun, S. Lyu, J. Mark. Polymer, 2001; 42:9783-9789.

13) Y. Yang, Z. Zhu, J. Yin, X. Wang, and Z. Qi. Polymer 1999;40:4407-4414.

14) M. Okamoto, S. Morita, H. Taguchi, Y. Kim, T. Kotaka, Polymer, 2000;41:3887.

15) Z. Wang, T. Pinnavaia. Chem. Mater., 10, 3769-3771.

16) Kresge, E.N., Lohse, D.J., 1996, US Patent 5,576,372.

17) Manias E., Conference Proceedings from Nanocomposites 2001, Chicago, Illinois.

18) H. Sun, G. Sur, G. Beaucage, and J. Mark. Polymer Prepreints, 2001;42(1):634.

19) R. Rothon. J Advances in Polymer Science, Mineral Fillers in Thermoplastics 1, 139, 1999. 


\section{Chapter II}

\section{Simple Mixing}

\section{Introduction}

Polyimide/organoclay nanocomposites are complex systems. Although the building blocks or the starting materials in these systems are well defined, combining the materials in a way that maximizes clay dispersion is difficult. There are numerous examples in which nanoclays were reported completely exfoliated ${ }^{\prime}$. The reports have been for all types of polymer systems including the polyimides. Many different combinations of mixing techniques and organoclays were used to delaminate the clay particles to create these disordered systems. The surfactant on the organoclay and the physical means by which the two components were brought in contact with one another were altered to create better dispersion in these materials. These two features are at the center of creating quality nanocomposites in any polymer system and are the focus of this chapter.

The earliest attempts at preparing polyimide nanocomposites involved simply mixing the poly(amide acid) (PAA) precursor and an organoclay suspension in solvent. This was reported in more than one instance as a successful method to prepare quality PAA solutions and hybrid nanocomposite materials. Successful dispersion of the organoclays was reported for both the organoclays modified with both aliphatic ${ }^{2-5}$ and aromatic ${ }^{6,7}$ surfactants. From a first glance this seemed improbable for two reasons. The aliphatic surfactant ion did not appear to be stable at $300^{\circ} \mathrm{C}$ and the aromatic organoclays that were being used appeared to be harder to disperse than aliphatic surfactant based organoclays. The work presented herein was a preliminary investigation into the reports 
of exfoliated nanocomposites. Some of the work was specifically aimed at repeating the procedures reported in the literature while other experiments were performed that were not directly repeating experiments in the literature. They all were based upon the principles for organoclay preparation and mixing set forth in the literature.

\section{Experimental}

\subsection{Starting materials}

The LaRC-8515 ${ }^{\mathrm{TM}}$ poly(amide acid) was purchased from Imitec, Inc. Ultem ${ }^{\mathrm{TM}}$ was purchased from General Electric. LaRC-CP1 $1^{\mathrm{TM}}$ and $\mathrm{LaRC}-\mathrm{CP} 2^{\mathrm{TM}}$ were also bought in the imide form from Imitec, Inc. The structures of the poly(amide acid)s, polyimides, and monomers are given below (see Figure 3).

The organically modified clay (SCPX-2003) used in the preparation of nanocomposite films was donated by Southern Clay Products, Gonzalez, TX and used as received. This organoclay consisted of a 2:1 montmorillonite with a cation exchange capacity (CEC) of $0.90 \mathrm{meq} / \mathrm{g}$ and was modified with a quarternary ammonium salt that contained a long chain hydrocarbon (tallow) group. Swy-2-Na+ montmorillonite was purchased from the mineral repository at the University of Missouri, Columbia. The synthetic hectorite was received from Corning and used as received. The following chemicals were obtained in polymer grade purity from the indicated sources and used as received: 1,3-bis(3-aminophenoxy) benzene [(APB), Mitsui Petrochemical Ind, Ltd, melting point (m.p.) 107-109 ${ }^{\circ} \mathrm{C}$ ]; pyromellitic dianhydride (PMDA), Aldrich , m.p. 283$286{ }^{\circ} \mathrm{C} ; 4,4^{\prime}$-oxydianiline [(ODA), Mitsui Petrochemical Ind, Ltd. m.p. 190-192 ${ }^{\circ} \mathrm{C}$ ]; 


\subsection{Preparation of TPP organoclays}

Into a 1000-mL Erlenmeyer flask was placed Swy-2 sodium montmorillonite (7.0109 g) and $650 \mathrm{~mL}$ of deionized water. The mixture was homogenized with a Powergen ${ }^{\mathrm{TM}}$ homogenizer for $\sim 5 \mathrm{~min}$ to insure good mixing. Into a separate $1000 \mathrm{~mL}$ beaker was placed TPP $(5.0091 \mathrm{~g})$ and $100 \mathrm{~mL}$ of deionized water. The mixture was stirred until the TPP dissolved. The clay was added to the aqueous TPP solution while homogenizing. The suspension was homogenized for another five minutes and subsequently transferred to a $1000 \mathrm{~mL}$ Erlenmeyer flask equipped with a condenser. The solution was held at $80^{\circ} \mathrm{C}$ for $12 \mathrm{hrs}$ while stirring. The clay was collected by vacuum filtration and was washed thoroughly with deionized water. The clay was dried in a vacuum oven at $70^{\circ} \mathrm{C}$ for $3 \mathrm{hrs}$ and stored in a low humidity chamber.

2.5. Preparation of controlled molecular weight poly(amide acid)s

The following is a sample calculation for preparing PAA solutions. The values that are placed in the table are the actual values used in the discussion below describing the synthesis of a $15,000 \mathrm{~g} / \mathrm{mol}$ polyimide. 
<smiles>Nc1ccc(Oc2ccc(N)cc2)cc1</smiles><smiles>O=C(c1ccc2c(c1)C(=O)OC2=O)c1ccc2c(c1)C(=O)OC2=O</smiles>

$322 \mathrm{~g} / \mathrm{mol}$<smiles>O=C1OC(=O)c2ccccc21</smiles>

$148 \mathrm{~g} / \mathrm{mol}$ $\underline{\text { value }}$

1.0
$8.0953 \mathrm{~g}$

grams added

$12.6100 \mathrm{~g}$

Amide acid repeat unit formula weight $=522.5 \mathrm{~g} / \mathrm{mol}$

Imide repeat unit formula weight $=486.5 \mathrm{~g} / \mathrm{mol}$

Table 1. Calculation of monomer addition amounts for controlled MW polyimide

In contrast to high molecular weight systems where a $1: 1$ stoichiometric ratio of each monomer is required, in order to adjust molecular weight the ratio of monomers needs to be adjusted. The dianhydride addition is offset by some amount and this makes the polymer chains shorter. An endcapper is added to the system to cap each chain with a non-reactive group thereby hindering further alteration of molecular weight. In order to calculate the ratio $(r)$ value of the individual monomers and endcapper some calculations 
need to be performed. Equations are shown below that are used to calculate the amount of each monomer and endcapper that are needed in the polymerization.

$$
\begin{gathered}
\overline{D P}=\frac{2(\text { CalculatedMolecularWeight })}{F W \text { Imide Re peatUnit }} \\
\mathrm{r}_{1} \text { (dianhydride) }=\frac{D P-1}{D P+1} \\
\mathrm{r}_{2} \text { (endcapper) }=2\left(1-\mathrm{r}_{1}\right)
\end{gathered}
$$

The first calculation that needs to be performed is the calculation of the degree of polymerization (DP). In the case of the polyimides discussed here, DP is the average number of diamine groups plus the number of dianhydride groups per polymer chain. The equation that can be used for this calculation is shown in equation 1. After determining the average degree of polymerization equation 2 can be used to calculate the molar ratio of dianhydride $\left(r_{1}\right)$ relative to the diamine added. Likewise equation 3 can be used to calculate the molar ratio of endcapper $\left(r_{2}\right)$ needed relative to the diamine. A more detailed discussion of the synthesis is described below.

The following example is representative of the procedure used to prepare neat poly(amide acid) solutions with controlled molecular weight.

Into a $100-\mathrm{mL}$ three-neck round-bottom flask equipped with a mechanical stirrer, nitrogen inlet, and drying tube containing calcium sulfate were placed ODA ( $8.0953 \mathrm{~g}$, 0.040 mole) and NMP $(25 \mathrm{~mL})$. The solution was stirred under nitrogen until the diamine completely dissolved $(\sim 0.5 \mathrm{hr})$. BTDA $(12.61 \mathrm{~g}, 0.039 \mathrm{~mole})$ was subsequently 
added followed by NMP $(25 \mathrm{~mL})$. Pthalic anhydride $(0.382 \mathrm{~g}, 0.003$ mole) was also added with NMP $(27.1 \mathrm{~mL})$ for a final solids content of $\sim 21 \%$. The mixture was stirred at room temperature for $\sim 24 \mathrm{hrs}$ under nitrogen. The resulting brown solution was clear and viscous. The solution was subsequently used in hybrid preparation as well as in the preparation of thin films for characterization.

\subsection{Preparation of high molecular weight poly(amide acid)s}

The following example is representative of the procedure used to prepare neat poly(amide acid) solutions.

Into a $100-\mathrm{mL}$ three-neck round-bottom flask equipped with a mechanical stirrer, nitrogen inlet, and drying tube containing calcium sulfate were placed ODA (6.4926 g, 0.032 mole) and NMP $(25 \mathrm{~mL})$. The solution was stirred under nitrogen until the diamine completely dissolved ( $0.5 \mathrm{hr})$. BTDA $(10.4480 \mathrm{~g}, 0.032$ mole) was then added followed by NMP ( $25 \mathrm{~mL}$ ) to adjust the solids content to $\sim 18 \%$. The mixture was stirred at room temperature for $\sim 24 \mathrm{hrs}$ under nitrogen. The resulting brown solution was clear and viscous. The solution was subsequently used to prepare thin films for characterization.

2.7. Unoriented thin films

Thin films were cast from neat (i.e. the control) poly(amide acid) and poly(amide acid)/organoclay mixtures in NMP. The solution was doctored onto clean, dry plate-glass 
and dried at room temperature to a tack-free form in a low humidity chamber. The films were subsequently dried in flowing air and characterized. The thermal conditions after air drying at room temperature were: (1) $100^{\circ} \mathrm{C}$ for $1 \mathrm{hr} ;(2) 1 \mathrm{hr}$ each at 100 and $200^{\circ} \mathrm{C}$; and (3) $1 \mathrm{hr}$ each at 100,200 , and $300^{\circ} \mathrm{C}$ to effect solvent removal and imidization. These thermal conditions were applied in both air and nitrogen. Film samples were removed from the plate glass and characterized. Thin-film tensile properties were determined according to ASTM D882 at room temperature using five specimens.

\subsection{Preparation of hybrids}

\subsubsection{Simple Mixing}

The following is a representative procedure used to prepare nanocomposites by mixing previously prepared poly(amide acid) solutions with clay solutions.

Into a $500 \mathrm{~mL}$ three-neck round-bottom flask equipped with a mechanical stirrer, nitrogen inlet, and drying tube containing calcium sulfate were placed ODA (6.0072 g, 0.030 mole) and NMP (50 mL). The solution was stirred until the diamine completely dissolved ( $0.5 \mathrm{hr})$. PMDA (6.5436 g, 0.030 mole) was added followed by NMP (50 $\mathrm{mL}$ ) to adjust the solids content to $\sim 11 \%$. The mixture was stirred at room temperature for $\sim 24$ hrs under nitrogen. The resulting brown poly(amide acid) solution was clear and viscous.

Into a 250-mL Erlenmeyer flask containing a magnetic stirbar were placed the organoclay (SCPX-2003, $2.9991 \mathrm{~g})$ and NMP (100 mL). The flask was fitted with a glass 
stopper. The contents were mixed at room temperature for $\sim 36$ hrs using a magnetic stirrer.

Into a separate 125-mL Erlenmeyer flask fitted with a glass stopper was placed the poly(amide acid) solution ( $20 \mathrm{~g})$. The clay solution (4.19 $\mathrm{g}$ ) was subsequently added while stirring continuously with a magnetic stirbar. This solution contained $\sim 5.6$ weight percent clay relative to the poly(amide acid). The resulting hybrid mixture was visually cloudy indicating significant phase separation. The mixture was subsequently used to prepare thin films that were characterized.

\subsubsection{Simple mixing with sonication}

The following is a representative procedure for the preparation of hybrid solutions by sonicating both the clay and hybrid solutions.

A hectorite-PPD organoclay prepared as described in Section 2.3 (1.7544 g) was placed in a 125-mL three-necked flask with NMP (50 mL). The organoclay/NMP mixture was stirred for $24 \mathrm{hrs}$ with an overhead stirrer and then was placed in a sonicator bath for 45 minutes. This clay solution $(1.7 \mathrm{~g})$ was added to a $250-\mathrm{mL}$ Erlenmeyer flask that contained a PAA solution of LaRC- $8515^{\mathrm{TM}}(9.84 \mathrm{~g}, 30 \%$ solids $)$ and mixed with a magnetic stirrer. The flask containing both the clay and polymer was immersed in a sonicator bath for 2 hours. During sonication the bath temperature reached $45-60{ }^{\circ} \mathrm{C}$. The resulting mixture was used to prepare films that were characterized by visual inspection. 


\subsubsection{Simple mixing with homogenization}

The following is a representative procedure for the preparation of polyimide/organoclay hybrids using high shearing forces produced by a homogenizer.

Into specialty glassware equipped with $\mathrm{N}_{2}$ inlet and calcium sulfate drying tube was placed SCPX-2003 (0.1006 g) and NMP (5.3475 g). The clay/NMP suspension was sonicated until no large clumps of clay were present. The suspension was then homogenized using a Powergen ${ }^{\mathrm{TM}}$ homogenizer for 5 minutes. After 5 minutes a solution of poly(amide acid) (15.6137 $\mathrm{g}, 21 \%$ solids, $15,000 \mathrm{~g} / \mathrm{mol})$ was slowly added while homogenizing. The mixture was homogenized for another 5 minutes yielding a yellowish brown ( $16 \%$ solids, $3 \%$ clay) solution. The many bubbles created during the homogenizing process were removed by centrifuging the mixture at $4000 \mathrm{rpm}$ for 10 minutes. Suspensions prepared in this manner were used to prepare unoriented thin films that were characterized.

\subsubsection{Melt Compounding and Extrusion}

a) Preparation of Ultem ${ }^{\mathrm{TM}}$ control samples

Ultem $^{\mathrm{TM}}$ pellets $(30 \mathrm{~g})$ were placed in a melt compounder and heated to $320^{\circ} \mathrm{C}$ while being stirred at $40 \mathrm{rpm}$ for $0.5 \mathrm{hr}$. After 5 minutes of stirring $15 \mathrm{~g}$ of Ultem ${ }^{\mathrm{TM}}$ was added to the mixture. Upon cooling, the solid was chopped to smaller pellets. The pellets were subsequently melted and extruded using a microextruder. The microextruder had two heating zones. The first was held at $450^{\circ} \mathrm{C}$ and the second 
was held at $585^{\circ} \mathrm{C}$. The mixer was rotated at $30 \mathrm{rpm}$ and the pressure reading was 3500 psi during the extrusion.

b) Preparation of $3 \%$ SCPX-2003/Ultem ${ }^{\mathrm{TM}}$ hybrid samples

Into a $250 \mathrm{~mL}$ polypropylene beaker were placed Ultem ${ }^{\mathrm{TM}}$ pellets $(30 \mathrm{~g})$ and SCPX-2003 $(0.69 \mathrm{~g})$. They were mixed in the beaker with a spatula and then placed in a melt compounder and heated to $320^{\circ} \mathrm{C}$ while being stirred at $40 \mathrm{rpm}$ for $0.5 \mathrm{hr}$. After 5 minutes of stirring $15 \mathrm{~g}$ of Ultem ${ }^{\mathrm{TM}}$ was added to the mixture. The mixture was subjected to the same procedure as in the example above.

c) Preparation of $3 \%$ Cloisite-Na+/Ultem ${ }^{\mathrm{TM}}$ hybrid samples

Into a $250 \mathrm{~mL}$ polypropylene beaker were placed Ultem ${ }^{\mathrm{TM}}$ pellets $(30 \mathrm{~g})$ and Cloisite-Na+ $(0.72 \mathrm{~g})$. They were mixed in the beaker with a spatula and then placed in a melt compounder and heated to $320^{\circ} \mathrm{C}$ while being stirred at $40 \mathrm{rpm}$ for $0.5 \mathrm{hr}$. After 5 minutes of stirring $15 \mathrm{~g}$ of Ultem $^{\mathrm{TM}}$ was added to the mixture. This mixture was also placed subjected to the same procedure as the neat Ultem ${ }^{\mathrm{TM}}$ sample.

\subsection{Other characterization}

Transmission electron microscopy (TEM) was obtained in the Department of Biology at William and Mary using a Zeiss EM109 electron microscope with an $80 \mathrm{kV}$ accelerating voltage. TEM samples were prepared by placing small strips of the sample film in an epoxy resin and curing the resin overnight at $80^{\circ} \mathrm{C}$. The samples were then cut using an ultramicrotome and placed on a 200 mesh copper grid for analysis. X-ray diffraction (XRD) patterns were obtained on polyimide films using a Scintag X1 X-ray diffractometer with a Copper $\mathrm{K}-\alpha(\lambda=0.15404 \mathrm{~nm})$ radiation source. 


\section{Results and Discussion}

\subsection{Preparation of organoclays}

Organoclays were prepared in ion-exchange reactions. All of the relevant ions were water-soluble and thus these reactions were done in aqueous systems. The ion exchange reactions were performed by mixing a $1 \%$ clay solution with a $5 \%$ salt solution. The reaction scheme that was used for this work involved adding a two-fold excess of the salt. The addition of an excess amount of the exchanging salt ensured exchange of the ion. Excess organic salt as well as the sodium chloride generated from the ion exchange reaction was removed by repetitive washing with deionized water. The structures of the ions used in the ion exchange reactions as well as the surfactant ion in SCPX-2003 are shown below in Figure 1.<smiles>[NH3+]c1ccc([NH3+])cc1</smiles>

PPD

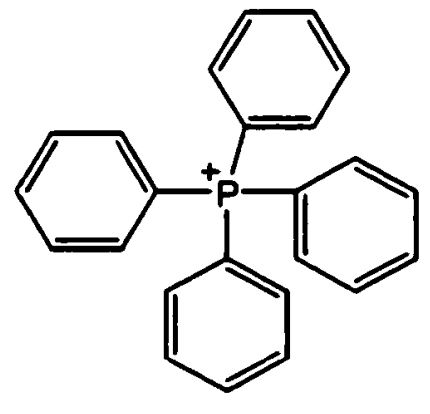

TPP<smiles>C[N+](CCO)(CCO)CCO</smiles>

SCPX-2003

Figure 1. Structure of ions used for ion exchange

\subsection{Preparation of polyimide-organoclay hybrids via simple mixing}

The ability of montmorillonite clays to expand beyond their natural dimensions can aid in their dispersion. Recent studies have shown that the introduction of a 
positively charged organic surfactant to the interlayer galleries (i.e. ion exchange), caused the negatively charged faces to separate to distances not found naturally. This replacement of inorganic cations with organic cations provides the surfaces of the silicate sheets with organic character and therefore facilitates the interaction with other organic substituents such as organic solvents and polymer molecules ${ }^{8-10}$. Consequently, polymer molecules are then able to enter the interlayer gallery (intercalation) of the once tightly packed, closely oriented clay sheets. The galleries can then expand beyond the point of any interaction with neighboring species resulting in a disordered array of individual clay particles (i.e. exfoliation). Unfortunately, the driving forces required for polymer molecules to enter the interlayer galleries are not generally favorable. In some cases more than one clay particle may be associated with the same surfactant molecule thus making exfoliation more difficult ${ }^{\prime \prime}$. In order to alleviate this problem the length of the non-polar portion of the surfactant is typically increased in order to increase the separation of clay sheets; thus long-chain aliphatic quaternary ammonium cations are typically used. The use of this type of surfactant ion has been successful in many polymer organoclay preparations reported in the literature. Although these modified organoclays work well in nanocomposite preparation, the aliphatic chains degrade at the high temperatures used to prepare polyimides ${ }^{14}$. The degradation of the surfactant is detrimental to the entire polymer system.

The degradation of the surfactant prompted immediate investigation into more thermally stable ions, particularly aromatic cations. It was suspected that these organoclays would not disperse as well as the aliphatic organoclays but the investigation into polyimide/organoclay began with the simple mixing of a variety of 
polyimide/organoclay combinations including both aliphatic and aromatic organoclays. Questions arose when preparing these systems concerning what mixing technique worked the best to disperse the organoclay. The focus quickly shifted from tailoring the building blocks to designing a better mixing process. The work presented below describes the results from different polymer/organoclay mixing techniques as well as the results from different combinations of polymer and organoclay. The way in which the polymer and organoclay were mixed varied from sample to sample but all followed the same general scheme followed depicted in Figure 2.

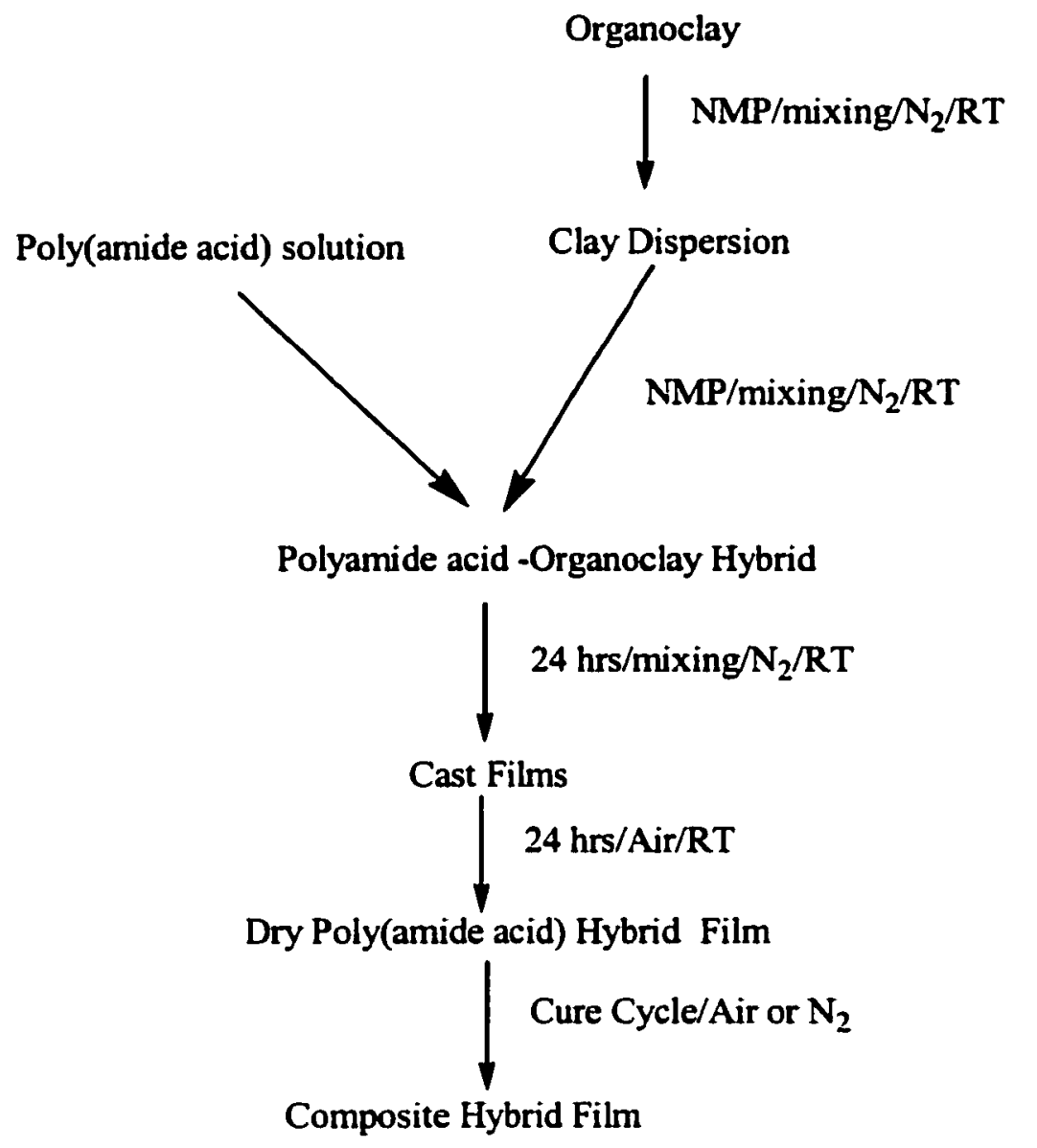

Figure 2. Flow chart of simple mixing technique 


\subsection{Preparation of PAA and polyimide solutions}

The PAA solutions were either purchased and used as received or were prepared according to the specifications discussed in Section 2 above. The PAA of LaRC-8515 was a PAA solution that was purchased and was a viscous brown solution prior to use. Ultem $^{\mathrm{TM}}, \mathrm{LaRC}-\mathrm{CP} 1^{\mathrm{TM}}$, and LaRC-CP2 ${ }^{\mathrm{TM}}$ are soluble in organic solvents in the imide form so they were purchased as imide powders. Ultem ${ }^{\mathrm{TM}}$ was received in small pellets while the $\mathrm{LaRC}-\mathrm{CP} 1^{\mathrm{TM}}$ and $\mathrm{LaRC}-\mathrm{CP} 2^{\mathrm{TM}}$ were received as small fibers. Polyimide solutions were prepared by dissolving the pellets or the fibers in organic solvent. The PAA solutions were prepared via the process in the experimental section. The structures of the monomers, the PAA, and the polyimides used in hybrid formation are shown below in Figure 3.<smiles>Nc1ccc(Oc2ccc(N)cc2)cc1</smiles>

ODA<smiles>O=C1OC(=O)c2ccccc21</smiles>

PA<smiles>O=C(c1ccc2c(c1)C(=O)CC2=O)c1ccc2c(c1)C(=O)OC2=O</smiles>

BTDA

PMDA<smiles>CCCCN1C(=O)c2ccc(C(F)(F)C(F)(F)F)cc2C1=O</smiles>

$\mathrm{LaRC}^{-C P I}{ }^{\mathrm{TM}}$ 


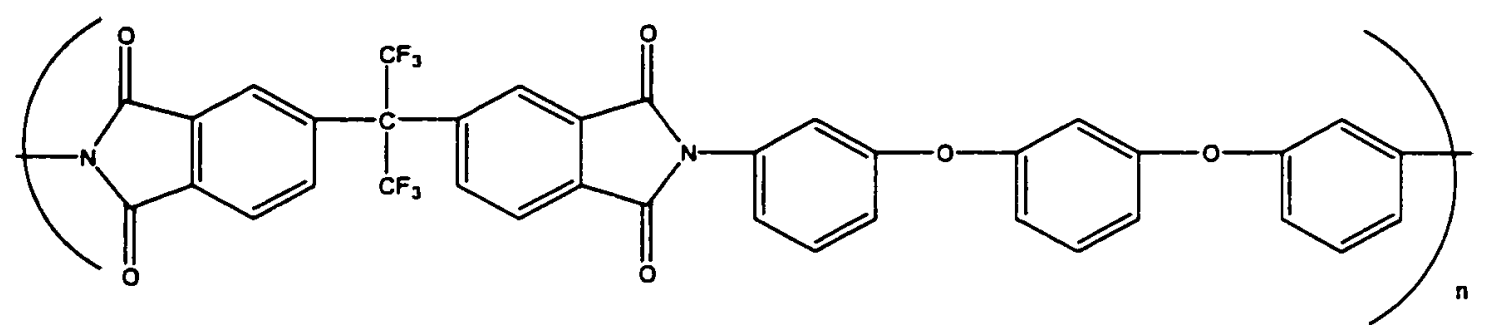

\section{LaRC-CP2 $^{\text {TM }}$}

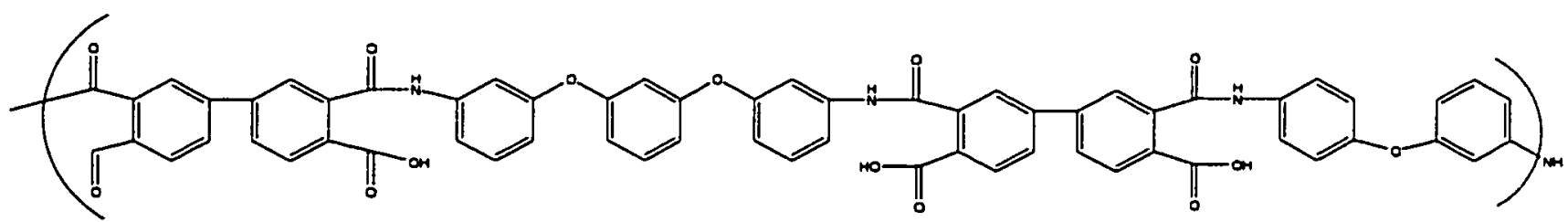

LaRC-8515 ${ }^{\mathrm{TM}}$ Poly(amide acid) precursor

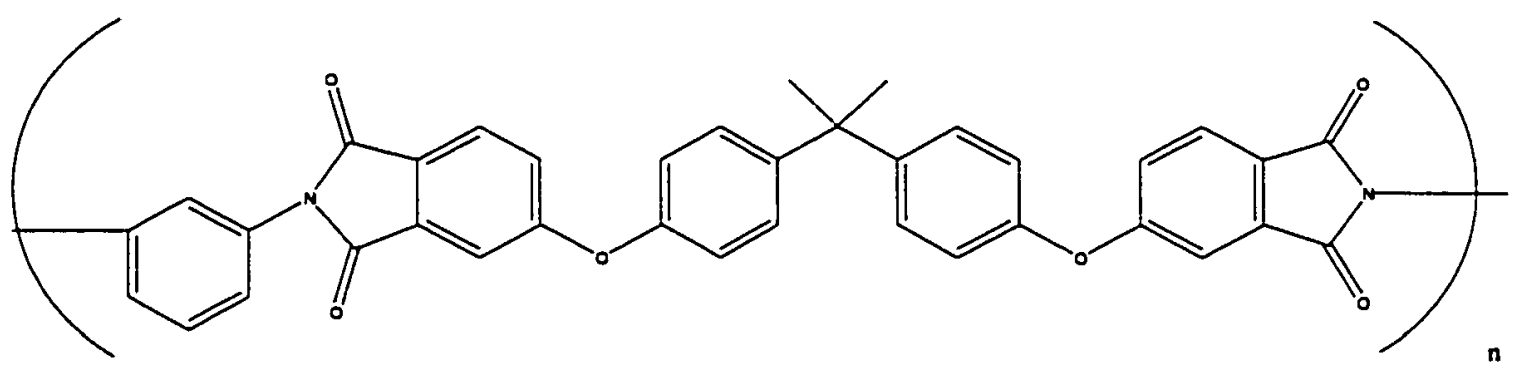

Ultem $^{\text {TM }}$

Figure 3. Chemical structures of monomers, PAA's, and polyimides

\subsection{Characterization of PPD organoclay/ODA-PMDA organoclay hybrids}

Many attempts were made at simply mixing PAA solutions with organoclays. One of the attempted combinations of PAA and organoclay was an ODA-PMDA polyimide with an organoclay that was modified with PPD (Section 2.3). This clay was prepared from the Swy-2-Na+ clay. Previous research with this combination found well dispersed clay 
particles that were reported as exfoliated ${ }^{2}$. The preparation of this material in our experiment involved mixing a high molecular weight poly(amide acid) with a clay suspension using a magnetic stir bar. The prepared mixture was cloudy, (i.e., not homogeneous). Films prepared from these solutions were also cloudy. Although the dispersion of the clay was not further characterized by TEM or XRD the mechanical properties of the hybrid material were examined. This was another way to examine nanocomposite preparations for their bulk properties and clay dispersion. Although generally not nearly as sensitive as XRD or TEM, it does offer a better picture of the bulk dispersion and bulk properties of a material. Both TEM and XRD give a picture of only a small area of the material. With mechanical testing a much larger area can be probed. The modulus of the materials gives information on the amount of surface area of the clay that is exposed in the sample. The modulus of a material usually increases with the addition of higher modulus fillers ${ }^{12}$. Although the modulus values usually increase, the toughness and $\%$ elongation of a material sometimes suffer as a result of the addition of a filler ${ }^{12}$. When preparing clay nanocomposites the clay particles tend to agglomerate into tactoids. These tactoids act as stress concentrators and greatly reduce the toughness and elongation properties of the material. The mechanical data obtained was used to give some rough indication as to how well the clay was dispersed.

Three different preparations had different concentrations of clay, but the same PAA solution was used for each. The mechanical property data for these materials and the control are shown below in table 2. 


\begin{tabular}{|c|c|c|c|}
\hline$\%$ Clay & Modulus (ksi) & Strength (ksi) & \% Elongation (\%) \\
\hline 0 & 296 & 16.3 & 40 \\
\hline 2.0 & 407 & 12.6 & 10 \\
\hline 5.0 & 400 & 13.9 & 13 \\
\hline 8.1 & 358 & 10.3 & 9 \\
\hline
\end{tabular}

Table 2. Mechanical properties of PPD organoclay/ODA-PMDA hybrids

The addition of clay increased the modulus in each preparation. Although all the hybrids had higher moduli than the neat polymer, finding the highest concentrations ( 5 and $8 \%$ ) with lower moduli than the $2 \%$ film was unexpected and different from those commonly reported for hybrid films. It appears that the films at the higher loading levels had clay that was so poorly dispersed that the mechanical properties did not perform in a normal manner at higher concentrations. These results were consistent with the higher concentration films being very cloudy, apparently the consequence of more agglomerates. The strengths of the hybrid films were lower than the strength of the neat film. The lower strength values may also be a result of clay agglomerates in the films. The effects of clay agglomerates are also manifest in the smaller elongation values of the clay-loaded films.

The agglomeration of clay in these preparations was clearly visible to the unaided eye. The mechanical properties suffered as a result of the failure to disperse the clay more completely. This agglomerate-polymer boundary is most likely the area where the polymer films break. Conclusion: this attempt to prepare nanocomposite materials was not successful.

3.7. Characterization of PPD organoclay /ODA-PMDA(offset) hybrids

Another approach at dispersing clay particles in the ODA-PMDA polymer system was examined. The same organoclay as in the above example was used but in this 
experiment a $1 \%$ stoichiometric excess of the dianhydride (PMDA) was included so that each polymer chain was terminated with dianhydride groups. Such chains would have a greater chance to react with the free amine on the PPD surfactant molecule and perhaps chains tethered to the clay particles would facilitate their dispersion. The movement of the chains in this type of system could accentuate the shearing forces to promote exfoliation. Mechanical properties of the system should also be enhanced for such a system. Hybrid mixtures were prepared via simple mixing techniques with ODA-PMDA, PAA and PPD-modified Swy-2 organoclay. The resulting mixtures, including the films, were similar to those prepared in the above example, i.e. cloudy with clay particles visually apparent. Again three preparations with differing concentrations of organoclay were prepared. The result of tensile tests of this simply mixed system are reported in Table 3.

\begin{tabular}{|r|r|r|r|}
\hline \% Clay & Modulus (ksi) & Strength (ksi) & \% Elongation (\%) \\
\hline 0 & 435 & 17.1 & 20 \\
\hline 2.7 & 453 & 18.8 & 20 \\
\hline 5.3 & 622 & 20.8 & 13 \\
\hline 11.3 & 403 & 12.7 & 10 \\
\hline
\end{tabular}

Table 3. Mechanical properties of PPD organoclay/ODA-PMDA(offset) hybrid The modulus data shown in Table 3 does show an increase in modulus up to $5 \%$. The film that had $11 \%$ clay exhibited a lower modulus than the neat resin. Later experimentation with the montmorillonite clays showed that optimal loading levels are those that are below $5 \%$. The strength data shown here indicates that there are some increases in strength at the lower clay loading levels. Again this was true except for the case where there was an overload of clay in the film with $11 \%$ clay. These data may give some indication that tethering is a good means to increase or at least maintain strength 
levels in clay/polymer systems. This was not a result of good dispersion of the clay particles because agglomerates could be seen in these films. The \% elongation in these films also showed an increase in the $2.65 \%$ clay loaded film but the elongation values decreased significantly in the higher loadings which once again points to clay agglomerates in the system.

This hybrid combination did not yield quality nanocomposites. The clay was agglomerated throughout the film but the mechanical properties did show some interesting trends. No further work was done with this material.

\subsection{Characterization of hectorite-PPD/ODA-PMDA hybrids}

Another combination of organoclay and polymer was studied in an effort to prepare a hybrid material having well dispersed clay particles. High molecular weight PMDAODA was used again but the base clay was changed from montmorillonite to hectorite. The hectorite was modified with PPD before being simply mixed with the PAA. The hybrid solution was opaque after simple mixing. Films were poured from the hybrid solutions and were cloudy as well. Three hybrid solutions were prepared from the initial PAA with varying amounts of organoclay and films were prepared from each. The mechanical results for the control and the simply mixed hybrid films are presented below.

\begin{tabular}{|r|r|r|r|}
\hline \% Clay & Modulus (ksi) & Strength (ksi) & \% Elongation (\%) \\
\hline 0 & 160 & 11.5 & 10 \\
\hline 2.9 & 201 & 6.28 & 7 \\
\hline 5.6 & 237 & 6.8 & 8 \\
\hline 9.3 & 213 & 7.25 & 9 \\
\hline
\end{tabular}

Table 4. Mechanical properties of hectorite PPD/ODA-PMDA hybrid

The modulus of these hybrids did increase as the clay loading was increased. Although all the films with clay had a higher modulus than the neat polymer there was a decrease 
as the loading went from $5 \%$ to $9 \%$. This was probably a result of a gross overload of clay in the $9 \%$ film. The increase at the 3 and $5 \%$ levels does indicate that these particles provide more stiffness to the materials. Conversely, the addition of these particles are detrimental to both strength and percent elongation. The strength values for the hybrid materials are all less than the value for the neat resin. This is not surprising as the clay was not well dispersed in this system. The clay agglomerates were visible without the aid of a microscope. Again the poor dispersion had a negative effect on these properties. The elongation of these materials is lower than in the case of the neat resin and again is a result of poor dispersion of the clay.

Changing the base clay used in these experiments did not greatly enhance the dispersion of the clay in these preparations.

\subsection{Characterization of TPP organoclay/ODA-PMDA hybrids}

Another combination of materials was simply mixed and the dispersion characteristics were examined. A high molecular weight ODA-PMDA polymer was mixed with organically modified montmorillonite. The Swy-2 clay was used in these experiments while the surfactant was changed to TPP. The tetraphenyl phosphonium ion, with phosphorous replacing nitrogen, was chosen due to the suspected high thermal stability of the aromatic ion and the large size. It was thought that the TPP would expand the clay layers more than the PPD. The expanded layers were also thought to have a better chance of exfoliating in the PAA solution. The resulting hybrid preparations were again cloudy and the films prepared from these solutions were cloudy films. The bulk 
properties of the material were measured by tensile testing and the mechanical data for these materials is presented below in Table 5 .

\begin{tabular}{|r|r|r|r|}
\hline \% Clay & Modulus (ksi) & Strength (ksi) & $\%$ Elongation (\%) \\
\hline 0 & 160 & 11.6 & 10 \\
\hline 2.38 & 293 & 10.8 & 10 \\
\hline 5.21 & 328 & 9.96 & 8 \\
\hline 11.14 & 168 & 3.41 & 5 \\
\hline
\end{tabular}

Table 5. Mechanical properties of TPP organoclay/ODA-PMDA hybrid films

The moduli for these hybrid films show that the addition of clay once again offered an increase in modulus over the neat polymer at all levels of loading. The $11 \%$ clay sample showed the lowest modulus value of the hybrid films. This again was probably the result of overloading the sample. Strength and elongation values for these materials decreased as the clay concentration was increased and is indicative of poor dispersion.

The hybrids prepared with this organoclay showed no real improvement over the other preparations discussed and were not examined further.

3.10. Characterization of hectorite-PPD/LaRC- $8515^{\mathrm{TM}}$ hybrids prepared via sonication

Different combinations of starting materials and mixing method were examined to determine if some combination of polyimide, organoclay, and mixing technique would result in dispersed nanoscopic clay particles in a polyimide matrix. One such combination was hectorite-PPD organoclay and $\mathrm{LaRC}-8515^{\mathrm{TM}}$. The $\mathrm{LaRC}-8515^{\mathrm{TM}}$ was purchased as a PAA at $30 \%$ solids content in NMP. A suspension of organoclay in NMP (3.5\% clay) was added to the PAA and the hybrid was mixed with a magnetic stirbar. Three different solutions were prepared containing 2,5 , and $8 \%$ clay by weight in the solution. The cloudy hybrid solutions were subsequently placed in a sonicator bath for 2 
hrs. The mixtures did not change much after sonication and the resulting solutions exhibited significant phase separation. Films were not prepared from these solutions and the solutions were not characterized.

\subsection{Characterization of SCPX-2003/ODA-PMDA hybrids}

To determine if an organoclay that was modified with an aliphatic quaternary amine would disperse in ODA-PMDA via simple mixing techniques, the two materials were mixed together. The high molecular weight PAA was mixed with a clay solution for a final clay concentration of $3 \%$. The solutions were mixed in a flask with a magnetic stirring bar. The solutions were brown and cloudy after mixing for $24 \mathrm{hrs}$. A film was prepared from this solution using the conventional thermal cure discussed above. The film had large agglomerates of organoclay in the film. The solution and the film were not further characterized for clay dispersion.

\subsection{Characterization of a low solids content PAA/organoclay hybrid}

The solids content of the polymer solutions used in simple mixing experiments were all above $10 \%$ solids. In an effort to prepare quality nanocomposites the solids content of a PAA was reduced to $5 \%$ solids prior to mixing with the clay solution. This method was reported as successful in preparing exfoliated polyimide/organoclay nanocomposites ${ }^{12}$. In this experiment an APB-BPDA PAA was used as the polymer matrix. The solution was not nearly as viscous as the solutions typically used for these mixtures. The PAA solution was mixed with the clay in a flask with a magnetic stirring bar. The solution seemed to mix thoroughly and there was no major phase separation. A film was prepared 
from this solution and thermally cured. The film had a grainy appearance and the clay was visible as agglomerates in the film. The film was not characterized by any other means.

3.13. Characterization of SCPX-2003/LaRC $-8515^{\mathrm{TM}}$ hybrids prepared via homogenization

The poly(amide acid) used in this experiment was an oligomer prepared at a calculated number average molecular weight of $9000 \mathrm{~g} / \mathrm{mol}$ designated LaRC-8515 ${ }^{\mathrm{TM}}$. The PAA was mixed with SCPX-2003 clay solutions using a Powergen ${ }^{\mathrm{TM}}$ homogenizer. The solutions as well as the films were clear after the mixing. This indicated that the clay was well dispersed in the PAA matrix. Mechanical properties were difficult to obtain due to the low molecular weight of the polymer. The films were very brittle and pieces large enough for mechanical testing were difficult to obtain. This preparation seemed to produce nanocomposites with well dispersed clay, but further characterization such as $\mathrm{XRD}$ showed that the clay was not completely exfoliated ${ }^{13}$.

\subsection{Characterization of SCPX-2003/LaRC-CP1 ${ }^{\mathrm{TM}}$ and SCPX-2003/LaRC-CP2 ${ }^{\mathrm{TM}}$} hybrids prepared via homogenization

LaRC-CP1 ${ }^{\mathrm{TM}}$ and LaRC-CP2 ${ }^{\mathrm{TM}}$ are both colorless polyimides. Both of these polyimides are soluble in organic solvents. These polyimides were chosen as the polymer matrix for use in nanocomposite preparation with SCPX-2003 organoclay. The polyimides were dissolved in NMP and mixed with a clay solution via homogenization. The solutions appeared clear after mixing. After sitting undisturbed for $12 \mathrm{hrs}$, the $3 \%$ 
clay solutions were poured to produce films. The films were yellow-brown rather than colorless after cure, a probable result of cation degradation in the organoclay. Although the films were colored they were clear and no visual clay particles were apparent. No further characterization was done with this material but it appeared that the homogenizer aided in dispersing the clay particles. This prompted further research with this mixing apparatus.

3.15. Characterization of SCPX-2003/ODA-BTDA hybrid films prepared via homogenization

To determine if simple mixing was enhanced by a high shear mixing technique an experiment was designed that compared hybrids prepared from simple mixing and high shear mixing. The XRD pattern for this organoclay is shown in Figure 4.

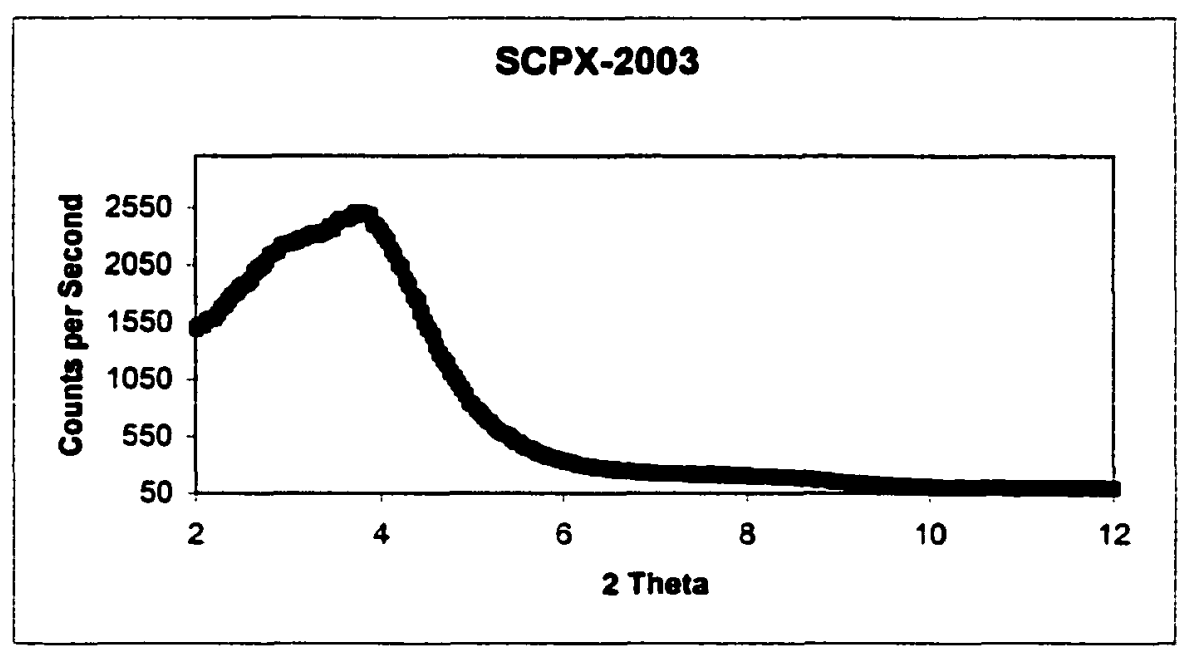

Figure 4. XRD pattern of SCPX-2003 organoclay

The XRD pattern indicates that all of the clay was ion exchanged and had a d-spacing of $2.37 \mathrm{~nm}$. Hybrid solutions were prepared in which the SCPX-2003 organoclay was homogenized before and after addition of the controlled molecular weight ODA-BTDA 
poly(amide acid) described in Section 2.5. The 3,5, and $8 \%$ films were clear after thermal cure. The films were darker and became more brittle at higher clay loadings. For example the $3 \%$ loaded film was amber and was fingernail creasable while the $8 \%$ film was almost black and was extremely brittle. The films were examined with XRD and the pattern for the 3,5, and $8 \%$ clay films are shown in Figure 5.

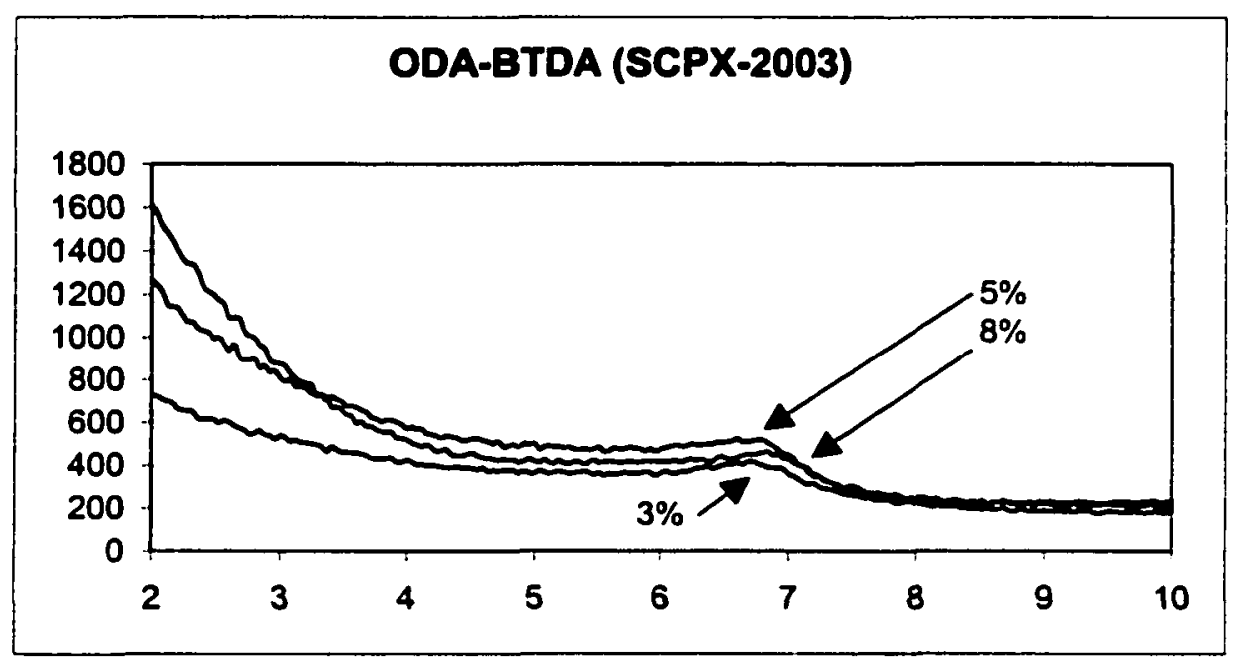

Figure 5. XRD pattern of homogenized SCPX-2003/ODA-BTDA hybrids

All of the polyimide films containing clay had a peak that can be attributed to one of two processes that take place during the curing cycle of the poly(amide acid). The peak position was roughly the same in all three cases but seemed to shift to a slightly higher angle at the higher clay loading levels. The d-spacings as calculated from the maxima in the XRD patterns were $1.32,1.30$, and $1.29 \mathrm{~nm}$ for the 3,5 , and $8 \%$ clay loaded films respectively. The percentage of clay that was agglomerated cannot be determined by XRD. The percentage is better determined by looking at the TEM micrograph (Figure 6). 

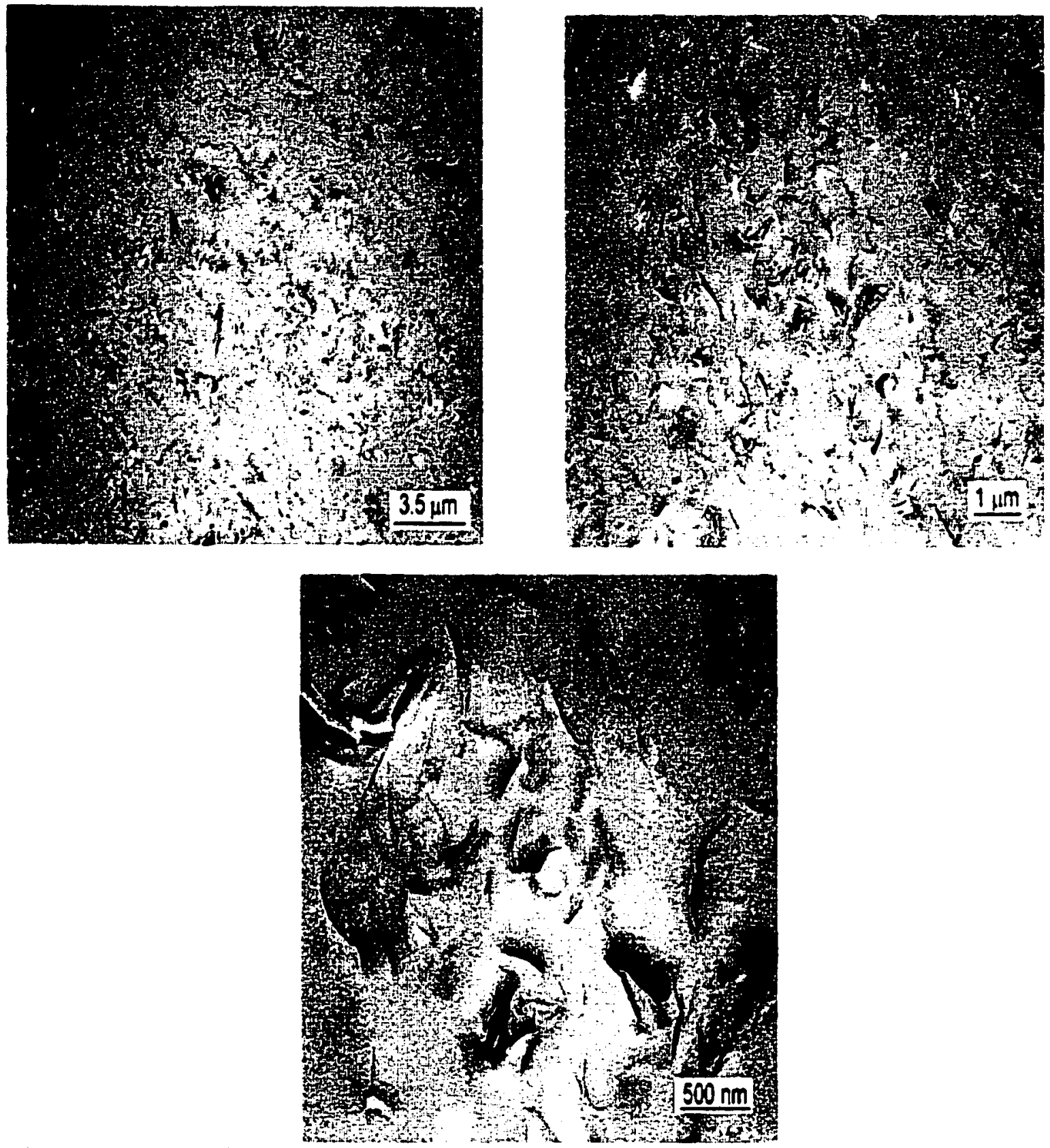

Figure 6. TEM micrograph of a 3\% SCPX-2003/ODA-BTDA polyimide prepared via homogenization

The TEM micrograph shows that the hybrid film had some exfoliated clay particles. In the lower magnification films there are areas of polymer where no clay is present, but these areas are small. This indicates that the clay is dispersed. In both the low and high magnification micrographs there are agglomerates present. Their presence is consistent 
with the XRD patterns. These agglomerates are thought to be clay particles that were not separated during the mixing process and after heating the surfactant degraded causing the particles to collapse. Either the particles were never separated by the shearing forces and after heating they collapsed, or the particles separated but agglomerated during cure. The position of the peak in the XRD pattern showed that the clay particles were actually closer together in the final film than in the original organoclay. This phenomona was studied with this particular clay and the results published ${ }^{14}$. Although there are some agglomerates present, the TEM micrographs showed that there are some individual clay particles as well. These particles seem to be exfoliated and seem to range from 200-700 $\mathrm{nm}$ in length. There seems to be approximately equal amounts of agglomerated and exfoliated clay particles in the film in the $20000 x$ magnified micrographs. The TEM micrographs cannot be used for a quantitative tool but they do show that the nanocomposite film had both agglomerates and exfoliated clay particles.

To determine if the homogenizer had an effect on dispersion, the results from the homogenization experiment were compared with a control in which everything was held constant except the homogenizing. In this preparation the same poly(amide acid) and the same clay was used but the clay was mixed with a conventional low shear overhead stirrer. The 3\% SCPX-2003/ODA-BTDA film was the only one prepared for comparison. The solutions prepared in this manner were not completely clear and were definitely not as clear as the homogenized sample. The films were also not as clear as the homogenized film after thermal cure. Small particulates could be seen in the film and were indicative of larger agglomerates. XRD patterns were also obtained for the polyimide films in which the poly(amide acid) solution was not subjected to high shear. 
The organoclay/polymer solution was simply mixed for 24 hours and a film was prepared from this solution. An XRD pattern was obtained for this film and shown in Figure 7.

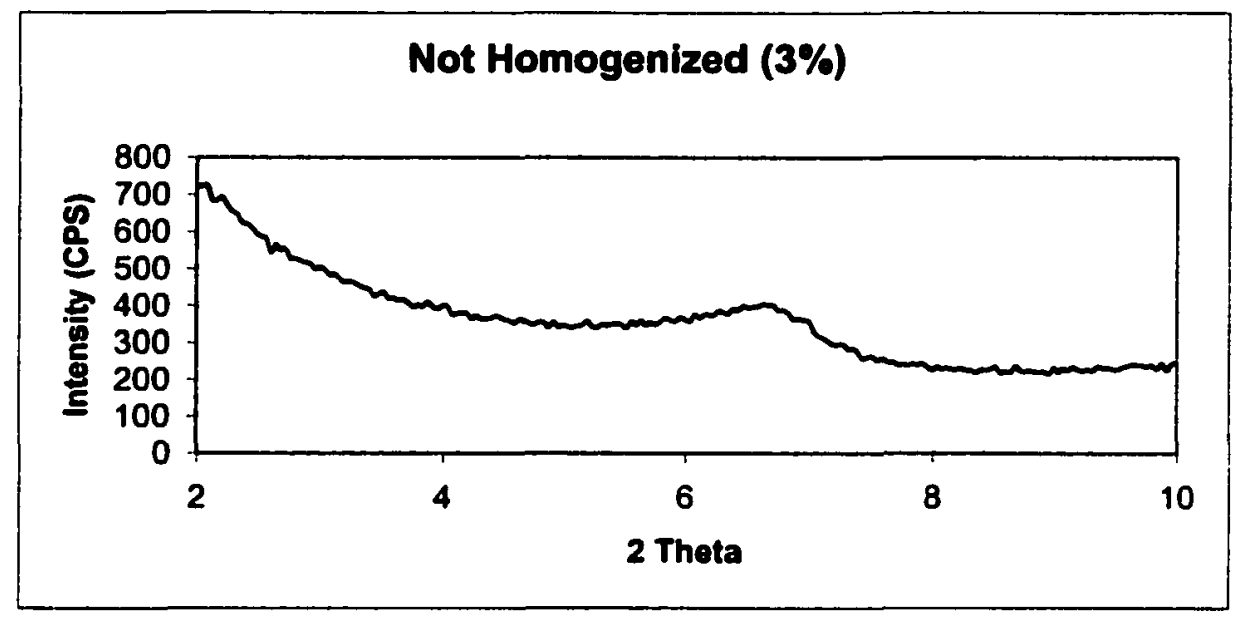

Figure 7. XRD pattern of simply mixed SCPX-2003/ODA-BTDA nanocomposites This XRD pattern also shows a peak indicating that some clay was not exfoliated. Again the percentage cannot be quantified but the d-spacing was determined to be $1.33 \mathrm{~nm}$. This d-spacing was consistent with that found for the homogenized samples but the dispersion in this film as determined by visual inspection was not nearly as good. Visually, the film was not as clear as the $3 \%$ film that was homogenized and larger agglomerates of clay could be seen throughout the polyimide film. This indicated that simple mixing was less effective than homogenization in dispersing particles. This is somewhat corroborated by the TEM micrographs of the $3 \%$ film shown below in Figure 8. 

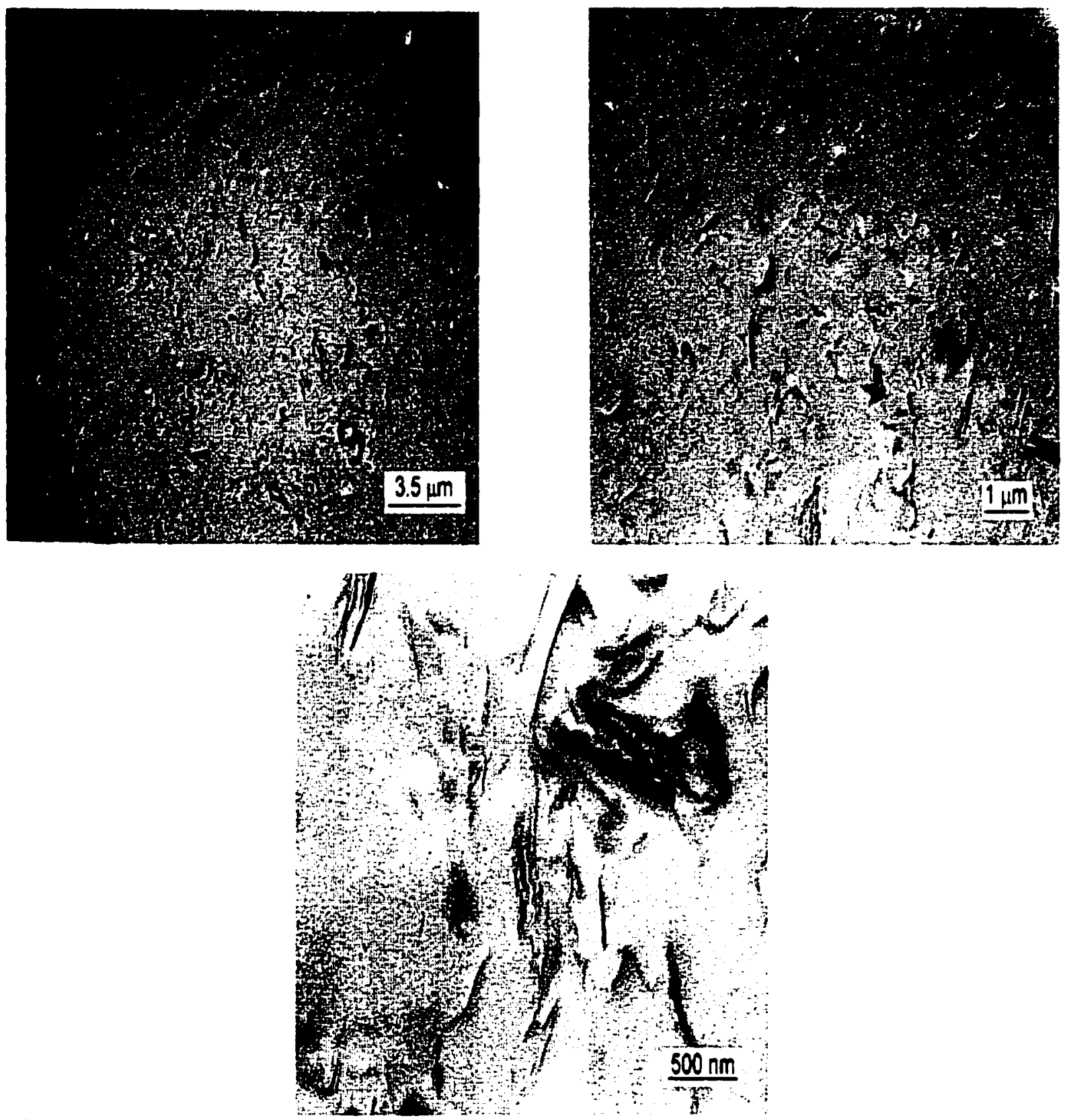

Figure 8. TEM micrograph of 3\% SCPX-2003/ODA-BTDA hybrid film prepared via simple mixing

Although there is relatively equal dispersion at the lower magnifications as in the homogenized film there is slightly more agglomeration apparent in the micrograph that is magnified 20000x. This gives additional evidence that the high shear mixing aided in dispersing the clay particles. The mechanical properties of this system are shown next. 
3.16. Mechanical Properties of SCPX-2003/ODA-BTDA hybrids prepared via homogenization

All three clay-containing films had significant increases in modulus over the neat material which indicates that the clay does add stiffness. Since simple mixing procedures resulted in materials with poor mechanical properties, high shearing forces were used on the both the organoclay solutions prior to mixing and on the polyimide/organoclay hybrid solutions. For this comparison phthalic anhydride endcapped ODA-BTDA polyimide was chosen as the matrix and had a calculated molecular weight of $15,000 \mathrm{~g} / \mathrm{mol}$. The modulus data for the neat polyimide film and 3,5 , and $8 \%$ clay loaded films are shown below in Figure 9.

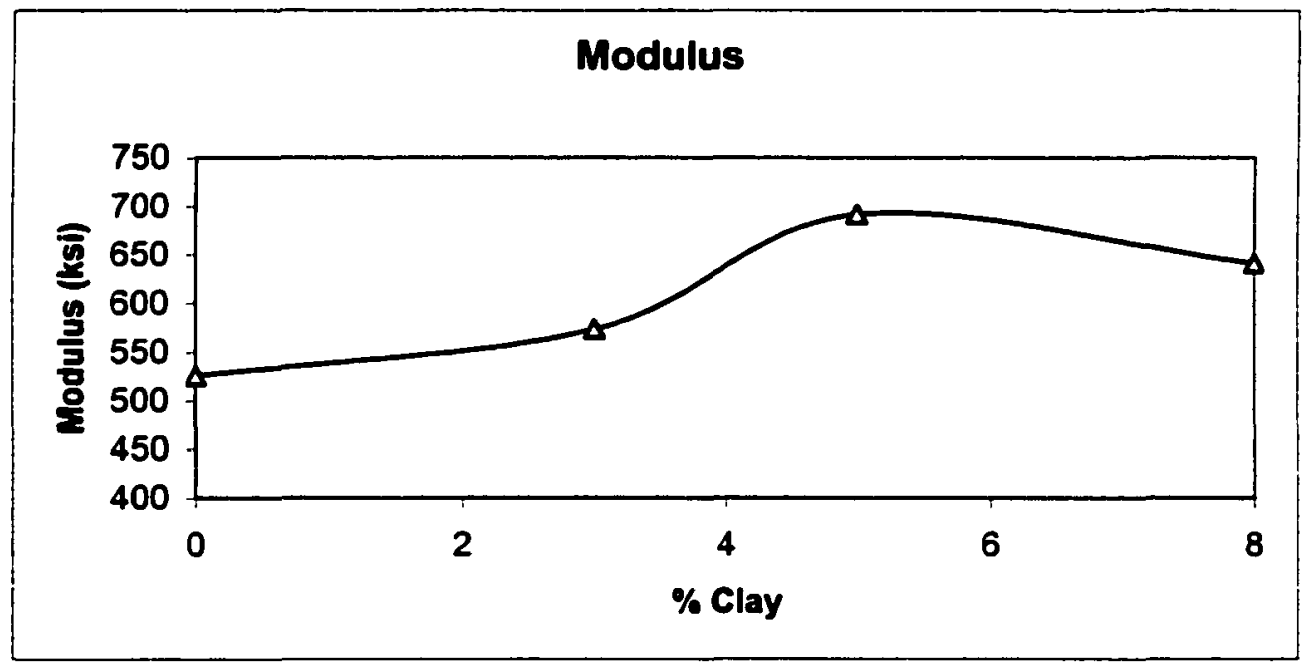

Figure 9. Modulus of SCPX-2003/ODA-BTDA (15000 g/mol) hybrids

The $3 \%$ film showed a significant increase, as the modulus is increased $50 \mathrm{ksi}$ at this loading. The 5\% film showed a large increase, as the modulus is $165 \mathrm{ksi}$ higher than the neat material. The modulus did not continue to increase in the $8 \%$ clay film dropped to $641 \mathrm{ksi}$. The data shown here are similar to modulus data of most polyimide/organoclay systems that are reported in later chapters. 
The strengths of these films decreased as the hybrid films were prepared with higher concentrations of clay. The strength values in these systems were also similar to those found in other polyimide/organoclay systems reported in later chapters. The strength data for this system are shown below in Figure 10.

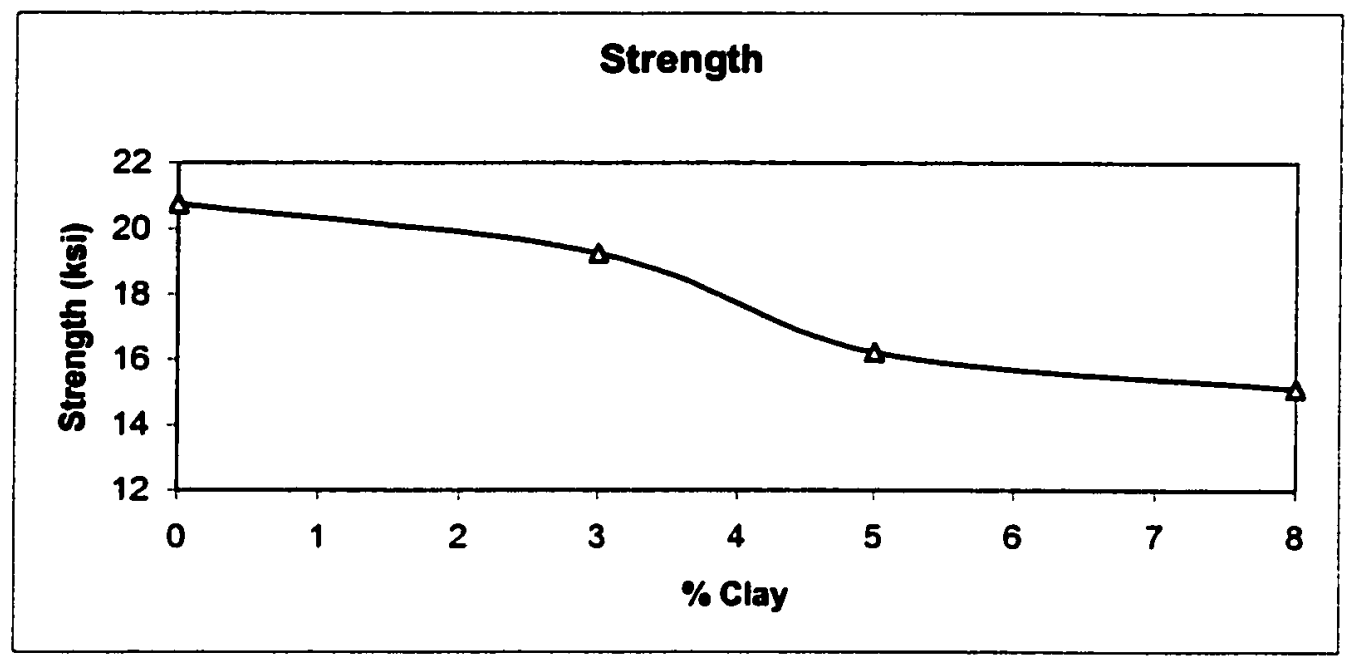

Figure 10. Strength of SCPX-2003/ODA-BTDA $(15000 \mathrm{~g} / \mathrm{mol})$ hybrids

The elongation of the films decreased with increasing amounts of clay in the system. The results are shown below in Figure 11.

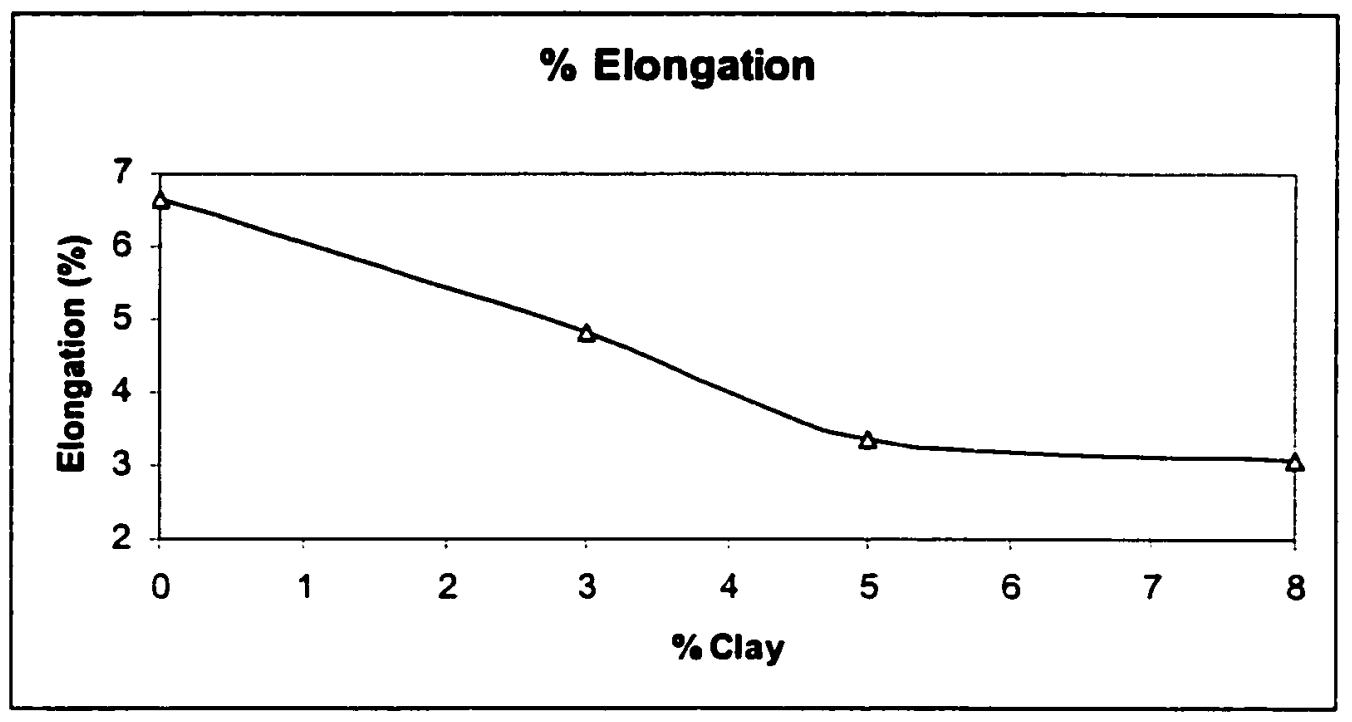

Figure 11. Elongation of SCPX-2003/ODA-BTDA (15000 g/mol) hybrids 
In summary, with the addition of clay, the stiffness went up at the expense of strength and elongation. This is indicative of a system where there were some well dispersed clay particles as well as some clay agglomerates. This is similar to the results determined with the other characterization techniques.

3.17. Characterization of Ultem ${ }^{\mathrm{TM}} /$ clay hybrids prepared via melt compounding and extrusion

A mixture of SCPX-2003 clay and Ultem ${ }^{\mathrm{TM}}$ were blended using a melt compounder (Section 2.8.4). The process was repeated for the Cloisite-Na+ and Ultem ${ }^{\mathrm{TM}}$. In addition neat Ultem ${ }^{\mathrm{TM}}$ was melted in the same compounder. It was clear, brown, and viscous. The Cloisite-Na+/Ultem ${ }^{\mathrm{TM}}$ mixture was darker brown and was not clear and the SCPX$2003 /$ Ultem $^{\text {TM }}$ mixture was black. In the case of the SCPX-2003, the black was most likely due to the degradation of the aliphatic surfactant present. On solidification, these materials were ground to small pellets. The pellets were placed in an extruder and melted at temperatures as high as $585^{\circ} \mathrm{C}$ and mixed with the polymer. The polymer was pushed through a small die and collected. The final product produced from the Cloisite$\mathrm{Na}+/$ Ultem extrusion was brown and opaque. The final product in the SCPX-2003/Ultem extrusion was black and opaque. Because the materials apparently suffered severe degradation, no further characterization was performed.

\section{Conclusion}

To determine the effect that different mixing techniques and surfactant ions have on the organoclay dispersion different mixing techniques and different starting materials 
were employed. Initially, simple mixing of clay, both organically modified and unmodified, with high molecular weight aromatic poly(amide acid)s yielded products that were not transparent. The films prepared from them contained clay agglomerates that were apparent to the unaided eye. Although tensile properties determined on thin films prepared by simple mixing it appeared that there were clay agglomerates that were detrimental to the strengths and elongation of the materials.

Consequently, another method to intercalate and exfoliate the clays in a polyimide matrix was investigated. This attempt involved the use of high frequency sound waves created in a sonicating water bath. The poly(amide acid) and organoclay mixtures were combined with a mechanical stirrer and then sonicated for up to 2 hours in an attempt to facilitate intercalation of the polymer into the clay galleries. These efforts proved futile as there was no increase in visual clarity of the mixture or the resulting polyimide films.

High shear mixing with shearing forces much greater than those typically encountered with a magnetic stirbar or mechanical stirrer improved intercalation and exfoliation. A Powergen ${ }^{T M}$ homogenizer imparted the high shear and the hybrid mixtures had improved clarity. Thin films of the PAA were cast and cured to $300^{\circ} \mathrm{C}$ in flowing air of both the neat polymer and the mixture. Visual inspection as well as TEM and XRD showed significant but incomplete exfoliation of the clay using this method of mixing. These materials were brittle making mechanical properties impossible to measure, but the materials were examined by Fourier transform infrared spectroscopy and $C^{13}$ nuclear magnetic resonance ${ }^{13}$. Additional work included high-shear mixing using the homogenizer with higher molecular weight polymer and high shear mixing using a melt compounder. The polymer/clay blends prepared using the compounder were not fully 
characterized because the high temperatures needed for this procedure caused the organoclay to degrade.

The organoclay's retention of an oriented structure in the presence of solvent and in the presence of poly(amide acid) proved to be a problem in the preparation of nanocomposites. It was clear that the clay and PAA were incompatible when simply mixed together. A partial solution was found with the use of high shearing forces. The materials prepared using simple mixing with homogenization (Section 2.8.3) seemed to contain the best dispersion of organoclay. Characterization showed that this mixing technique was not completely effective and clay agglomerates remained as evidenced by both XRD and TEM. The mechanical properties of the material suffered as a result.

It was clear from this early research that a more thorough investigation of nanocomposite materials was required. How the components are mixed is a key feature in preparing quality nanocomposites. Other features such as the choice of surfactant and clay also play a role. These insights in the preparation of polyimide/organoclay nanocomposites led to a more detailed approach to the problem. 


\section{Chapter II References}

1) P. Lebaron, Z. Wang, T. Pinnavaia. Applied Clay Science 1999;15:11.

2) H. Tyan, Y.Liu, K.Wei. Polymer 1999;40:4877.

3) K. Yano, A. Usuki, A. Okada, T. Karauchi, O. Kamigato. J. Poly Sci., Part A Poly. Chem., 1993;31:2493

4) K. Yano, A. Usuki, A. Okada. J. Poly Sci., Part A Poly. Chem. 1997;35:2289

5) T. Lan, P.Kavirtna, T. Pinnavaia, J. Cem. Mater. 1994;6:573.

6) H. Tyan, K.Wei, T. Hsieh. J. Poly. Sci., Part B, Poly, Phys. 2000;38:2873.

7) H. Tyan, K.Wei, T. Hsieh. Chem. Mater. 1999;11:1942.

8) A. Usuki, M. Kawasumi, Y. Kojima, A. Okada, T. Karauchi, O. Kamigato. J. Mater. Res., 1993;8:1174.

9) A. Usuki, Y. Kojima, M. Kawasumi, Y. Fukushima, T. Karauchi, O. Kamigato. J. Mater. Res., 1993;8:1179.

10) Y. Kojima, A. Usuki, A. Okada, M. Kawasumi, Y. Fukushima, T. Karauchi, O. Kamigato. J. Mater. Res. 1993;8:1185.

11) R. Rothon, J Advances in Polymer Science, Mineral Fillers in Thermoplastics 1, 139, 1999.

12) T. Agag, T. Koga, T. Takeichi. Polymer 2001:3399.

13) S. Zheng, R.L. Vold, G.L. Hoatson, and D. Delozier. Polymer, manuscript in preparation.

14) D. Delozier, R. Orwoll, J. Cahoon, N. Johnston, J. Smith, and J. Connell. Polymer 2002;43:813. 


\section{Chapter III}

\section{In-situ polymerization with low temperature organoclays}

\section{Introduction}

Certain organically modified clays comprised of silicate sheets separated by cations can be homogeneously incorporated into organic polymers to enhance select physical, morphological, and mechanical properties ${ }^{1,2}$. A hybrid inorganic-organic material is produced with altered properties that vary depending on the level of dispersion, the ammonium cation, the silicate, and the host polymer ${ }^{1-18}$. One example by Pinnavaia et. al. is the incorporation of low concentrations of organically modified silicate sheets (organo-montmorillonite) into a polyimide that improved certain mechanical and thermal properties $^{3-6}$. Visually clear films, indicative of a dispersion of nanometer-sized particles, were obtained with lower coefficient of thermal expansion (CTE), markedly lower gas permeability coefficients, and higher mechanical properties than the pristine material. The lower gas permeability coefficient is attributed to the dispersed clays creating a tortuous path for gas molecules ${ }^{19}$. Other property enhancements such as reduced time and temperature to effect imidization ${ }^{20}$ and increased resistance to certain solvents ${ }^{21}$ were manifest in polyimide systems with dispersed nanoclays. The polyimide/organoclay nanocomposites in these examples were all prepared via simple mixing. Although the simple mixing technique was proposed by these researchers as a viable blending approach, the work presented in chapter II revealed that the simple mixing technique was not very effective at dispersing organoclays. The work presented herein was focused on 
developing a better method to prepare well-dispersed polyimide/organoclay nanocomposites. An in-situ polymerization approach was used to facilitate clay dispersion. Different low-temperature organoclays and polyimides were evaluated to find a polyimide/organoclay combination in which a high degree of dispersion was achieved as evidenced by the characterization of physical and mechanical properties of nanocomposite films. Since the organically modified clays typically contain long chain aliphatic hydrocarbon based cations, incorporation of these materials into high temperature polymers such as polyimides is of concern due to the potential for thermal decomposition of the cation during high temperature processing. Investigations of these organoclays using thermogravimetric analysis/mass spectroscopy revealed that long and short chain carbon species evolved at approximately $200^{\circ} \mathrm{C}$ and $300^{\circ} \mathrm{C}$, respectively ${ }^{22}$. Since the preparation of the films used a high-temperature treatment (i.e. $1 \mathrm{hr}$ each at 100 , 200 and $300{ }^{\circ} \mathrm{C}$ in air and nitrogen), the effects of thermal treatment on the physical and mechanical properties as well as morphological changes resulting from the thermal decomposition of the aliphatic organic cation were also investigated.

\section{Experimental}

\subsection{Starting materials}

The organically modified clay (SCPX-2003) was used in the preparation of nanocomposite films. Cloisite $(\mathrm{Na}+)(\mathrm{CEC}=0.90 \mathrm{meq} / \mathrm{g})$ montmorillonite was obtained from Southem Clay Products and used as received. BK3-166A, BK3-166B, BK3-166C and BK3-166D were donated by Triton Sytems, Chelmsford, MA and used as received. 
The following chemicals were obtained in polymer grade purity from the indicated sources and used as received: APB, PMDA, ODA, BPDA, BTDA, oxydiphthalic dianhydride [(ODPA), Imitec, Inc., m.p. 224-225.5 $\left.{ }^{\circ} \mathrm{C}\right]$; 4,4'-perfuoroisopropylidene dianhydride [(6FDA), Hoechst Celanese Inc., m.p. 241-243 $\left.{ }^{\circ} \mathrm{C}\right]$; 3,3'diaminobenzophenone [(BPD), Mitsui Toatsu Chemicals, Inc.]; 3,3'-diaminodiphenyl sulfone [(DDS), FIC Corporation, Resins Division]; and [2,4-bis(3aminophenoxy)phenyl]-diphenylphosphine oxide [(3-APPO), NASA-LaRC, m.p. 192$\left.196.5^{\circ} \mathrm{C}\right]$.

High purity, anhydrous NMP was purchased from Aldrich and used as received.

\subsection{Preparation of DTMA organoclays}

The following is a representative procedure used to prepare organically modified montmorillonite clays.

Into a $250-\mathrm{mL}$ fleaker was placed Cloisite $\mathrm{Na}+(1.0001 \mathrm{~g})$ and $100 \mathrm{~mL}$ of deionized water. The clay was mixed with a homogenizer until a stable suspension was reached. In a $25-\mathrm{mL}$ beaker was placed dodecyl trimethyl ammonium bromide $(0.2385 \mathrm{~g})$ and $20 \mathrm{~mL}$ of deionized water. The salt/water suspension was stirred with a magnetic stir bar at 60 ${ }^{\circ} \mathrm{C}$ until the salt dissolved. The clay suspension was added slowly to the salt solution while homogenizing. The clay/salt suspension was homogenized for approximately five minutes to ensure good mixing. The entire mixture was then transferred to a $1000-\mathrm{mL}$ round bottom flask equipped with a condenser, and $100 \mathrm{~mL}$ of deionized water was added. The suspension was stirred with a magnetic stirbar and the temperature was 
maintained at $80{ }^{\circ} \mathrm{C}$ with a heating mantle for $3 \mathrm{hrs}$. The apparatus was assembled and the suspension was poured through a Soxhlet thimble and allowed to drain. The clay in the thimble was extracted for $24 \mathrm{hrs}$ with a 50/50 (v/v) water/ethanol solution, transferred to a quartz crucible, and heated at $85^{\circ} \mathrm{C}$ for $24 \mathrm{hrs}$. Finally, the clay was ground to a fine powder with a mortar and pestle.

\subsection{Preparation of DTPP organoclays}

The following is a representative procedure for preparing organically modified montmorrillonite clays.

Into a $250-\mathrm{mL}$ fleaker was placed Cloisite $\mathrm{Na}+(1.0001 \mathrm{~g})$ and $100 \mathrm{~mL}$ of deionized water. The clay was homogenized until a sol was reached. In a separate $500-\mathrm{mL}$ beaker was placed dodecyl triphenyl phosphonium bromide $(1.0104 \mathrm{~g})$ and $200 \mathrm{~mL}$ of deionized water. The clay suspension was added slowly to the salt solution while homogenizing. The remaining preparation followed the procedure described in Section 2.2.

\subsection{Preparation of poly(amide acid)s}

Refer to Section 2.6 in chapter II.

2.5. Preparation of poly(amide acid)/organoclay hybrids via in-situ polymerization The following example is representative of the procedure used to prepare poly(amide acid)/organoclay mixtures by synthesizing the poly(amide acid)s in the presence of the clay suspension. 
Into a $100 \mathrm{~mL}$ volumetric flask were placed SCPX-2003 (0.5095 g) and NMP (25 $\mathrm{mL}$ ). The flask was fitted with a glass stopper and placed on a mechanical shaker for $\sim 1$ hr at room temperature. The suspension was cloudy, but no large clusters or clumps of clay were visually observed. Into a $100 \mathrm{~mL}$ three-neck round-bottom flask equipped with a mechanical stirrer, nitrogen inlet, and drying tube containing calcium sulfate were placed the organoclay suspension, ODA $(6.4983 \mathrm{~g}, 0.032 \mathrm{~mole})$ and NMP $(25 \mathrm{~mL})$. The mixture was stirred under nitrogen until the diamine completely dissolved $(\sim 0.5 \mathrm{hr})$. BTDA (10.4572 g, $0.032 \mathrm{~mol})$ and NMP $(25 \mathrm{~mL})$ were subsequently added. The mixture was stirred at room temperature for $\sim 24 \mathrm{hrs}$ under nitrogen. The solution concentration was $\sim 18 \%$ solids with a clay concentration of $\sim 3 \%$. The resulting brown mixture was clear and viscous. The mixture was used to prepare thin films for characterization.

\subsection{Unoriented thin films}

Thin films were cast from neat (i.e. control) poly(amide acid) and poly(amide acid)/organoclay mixtures in NMP. The solution or mixture was doctored onto clean, dry plate-glass and dried at room temperature to a tack-free form in a low humidity chamber. The films were subsequently treated under several different drying conditions in flowing air and characterized. The thermal conditions after air drying at room temperature were: (1) $100{ }^{\circ} \mathrm{C}$ for $1 \mathrm{hr}$; (2) $1 \mathrm{hr}$ each at $100^{\circ} \mathrm{C}$ and $200^{\circ} \mathrm{C}$; and (3) $1 \mathrm{hr}$ each at 100,200 , and $300{ }^{\circ} \mathrm{C}$ to effect solvent removal and imidization. These thermal conditions were applied in both air and nitrogen. Film samples were removed from the plate glass and characterized. Thin-film tensile properties were determined according to ASTM D882 at room temperature using five specimens. 


\subsection{Other characterization}

Thermogravimetric analysis (TGA) was performed on a Seiko SSC/5200 thermal analyzer at a heating rate of $2.5^{\circ} \mathrm{C} / \mathrm{min}$ in air and nitrogen at a flow rate of $50 \mathrm{~mL} / \mathrm{min}$. Differential scanning calorimetry (DSC) was performed using a TA instruments DSC 2900 at a heating rate of $10^{\circ} \mathrm{C} / \mathrm{min}$. with the glass transition temperature $(\mathrm{Tg})$ taken at the inflection point of the $\Delta \mathrm{H}$ versus temperature curve. Transmission electron microscopy (TEM) was obtained using a Zeiss EM109 electron microscope with an 80 $\mathrm{kV}$ accelerating voltage. TEM samples were prepared by placing small strips of the sample film in an epoxy resin and curing the resin overnight at $80^{\circ} \mathrm{C}$. The samples were then cut using a ultramicrotome and placed on a 200 mesh copper grid for analysis. Xray diffraction (XRD) patterns were obtained on solutions of poly(amide acid) in NMP, poly(amide acid) films, and polyimide films using a Scintag X1 X-ray diffractometer with a Copper $\mathrm{K}-\alpha(\lambda=0.15404 \mathrm{~nm})$ radiation source.

\section{Results and Discussion}

\subsection{Preparation of organoclays}

The majority of the nanocomposites described in this chapter incorporated SCPX2003 organoclay. Although this was the clay that was studied the most it was not used exclusively. However, some experiments were performed with other low temperature organoclays. Some of these organoclays were received from Triton while other organoclays were prepared via ion exchange reactions. The procedure for preparing 
organoclays is presented in the experimental section. The structures of the surfactant ions present in the organoclays are presented in Figurel.<smiles>C[N+](C)(CCO)CCO</smiles>

SCPX-2003<smiles>CCCCCC</smiles>

DTMA<smiles>CCCCN(c1ccccc1)c1ccccc1</smiles><smiles>C[N+](CCO)(CCO)CCO</smiles>

BK3-166A<smiles>CCCCC[P+](C)(C)C</smiles>

BK3-166C<smiles>C[N+](CCO)(CCO)CCO</smiles>

BK3-166B*<smiles>CCCC[P+](C)(C)C</smiles>

BK3-166D*

Figure 1. Structures of surfactant ions

* The organoclay was also treated with $3 \%$ an undisclosed amino silane.

3.2. Preparation of poly(amide acid)/organoclay solutions via in-situ polymerization

The previous chapter was dedicated to discussing the results of nanocomposite preparations using simple mixing techniques. The simple mixing technique was not 
effective for preparing polyimide nanocomposites. Yang et $\mathrm{al}^{23}$ proposed an improvement in which the polymerization was carried out in the presence of the clay. They reported good clay dispersion in some cases. Initially, the diamine was combined with the NMP/clay mixture with the intercalation of the diamine into the interlayer gallery of the clay. Subsequent addition of the dianhydride afforded solutions that exhibited only slight cloudiness. A representative procedure for preparing the hybrid nanocomposites is represented in Figure 2.

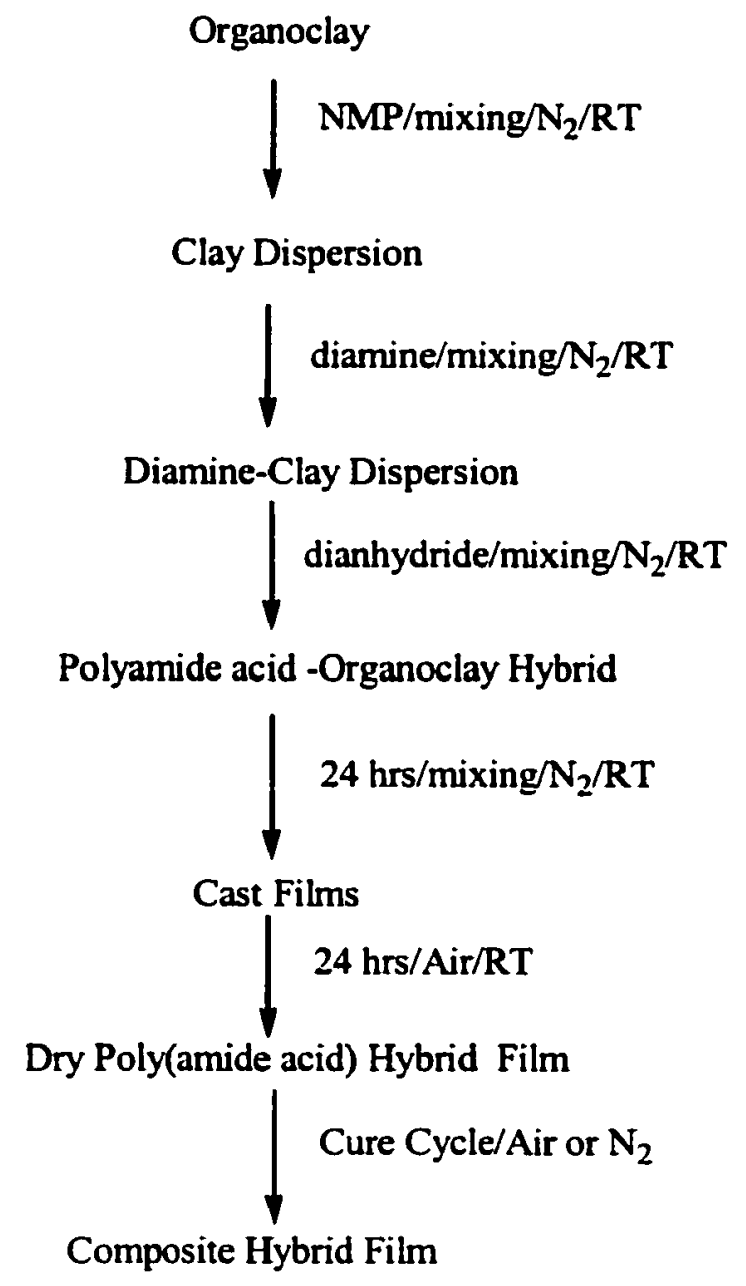

Figure 2. Flow chart of hybrid preparation 
With this approach, at least a portion of the polymer could reside in clay gallery. The growth of the polymer, with its origin being in the gallery, could provide additional aid in forcing the clay layers apart resulting in exfoliation. The chemical structures of the monomers used in this study are depicted in Figure 3.<smiles>Nc1cccc(Oc2cccc(Oc3cccc(N)c3)c2)c1</smiles>

APB<smiles>O=C1OC(=O)c2cc(-c3ccc4c(c3)C(=O)OC4=O)ccc21</smiles>

BPDA<smiles>Nc1ccc(Oc2ccc(N)cc2)cc1</smiles>

ODA<smiles>O=c1oc(=O)c2cc3c(=O)oc(=O)c3cc12</smiles><smiles>O=C(c1ccc2c(c1)C(=O)OC2=O)c1ccc2c(c1)C(=O)OC2=O</smiles><smiles>Nc1cccc(C(=O)c2cccc(P)c2)c1</smiles> 
<smiles>Nc1cccc(S(=O)(=O)c2cccc(N)c2)c1</smiles>

DDS<smiles>CC([18O])(c1ccc2c(c1)C(=O)OC2=O)c1ccc2c(c1)C(=O)OC2=O</smiles>

6-FDA

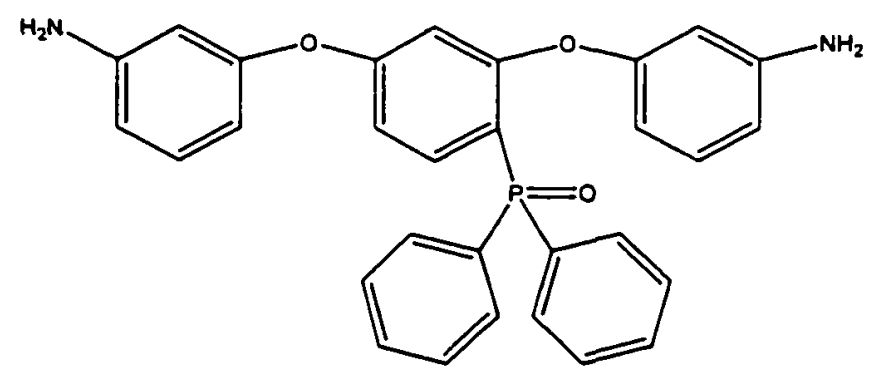

\section{3-APPO}

Figure 3. Monomers used to prepare polyimides

The hybrid solutions were used to cast thin films. Four films were prepared from the solutions that were prepared with SCPX-2003/APB-BPDA and SCPX-2003/ODABTDA. These films received several different thermal treatments prior to characterization. These included air-drying at room temperature in a low humidity chamber with no further thermal treatment. In addition, the air-dried films were thermally treated: (1) $100^{\circ} \mathrm{C}$ for $1 \mathrm{hr}$; (2) $1 \mathrm{hr}$ each at 100 and $200^{\circ} \mathrm{C}$; and (3) $1 \mathrm{hr}$ each at 100,200 , and $300^{\circ} \mathrm{C}$. All other films just received one thermal treatment; $1 \mathrm{hr}$ each at 100,200 , and $300^{\circ} \mathrm{C}$. All thermal treatments were performed in a forced air and forced nitrogen ovens. No visual difference between the pristine films and the ones containing $3 \%$ clay were observed for APB-BPDA based films treated for $1 \mathrm{hr}$ at $100^{\circ} \mathrm{C}$ in both air and nitrogen. These clay-containing films began to darken after treatment for $1 \mathrm{hr}$ each at 100 and $200{ }^{\circ} \mathrm{C}$. Films that were thermally treated in air began to turn a light brown whereas the same compositions treated in nitrogen changed to a light yellow. After 
thermal treatment to achieve solvent removal and imidization (i.e. $\mathrm{l} \mathrm{hr}$ each at 100, 200 and $300^{\circ} \mathrm{C}$ ), the films were darker in color as compared to control films treated under the same thermal conditions. Once again the use of forced air resulted in dark brown films while films thermally treated under nitrogen became yellow. Pristine films thermally treated under nitrogen to effect imidization were less colored than those treated in air. Coloration was more apparent in films containing higher clay-loading levels (i.e. $5 \%$ and 8\%). The control film obtained from ODA-BTDA was clear and brown after thermal imidization in air, while the film containing $8 \%$ clay was considerably darker and nearly black. Thermally imidized films from APB-BPDA exhibited a similar behavior.

The neat polyimide films and films containing up to $3 \%$ clay were tough and fingernail creasable, while the higher clay content films were brittle and uncreasable. This brittleness may be a consequence of the higher clay loading or the formation of agglomerates as a result of the thermal decomposition of the organic cation.

3.3. Analysis of 3\% SCPX-2003 polyimide blends prepared via in-situ polymerization

Many different poly(amide acid)s were prepared in the presence of SCPX-2003 to determine if the organoclay/polyimide combination would yield nanocomposites with high levels of clay dispersion. The results of these experiments are listed in Table 1. 


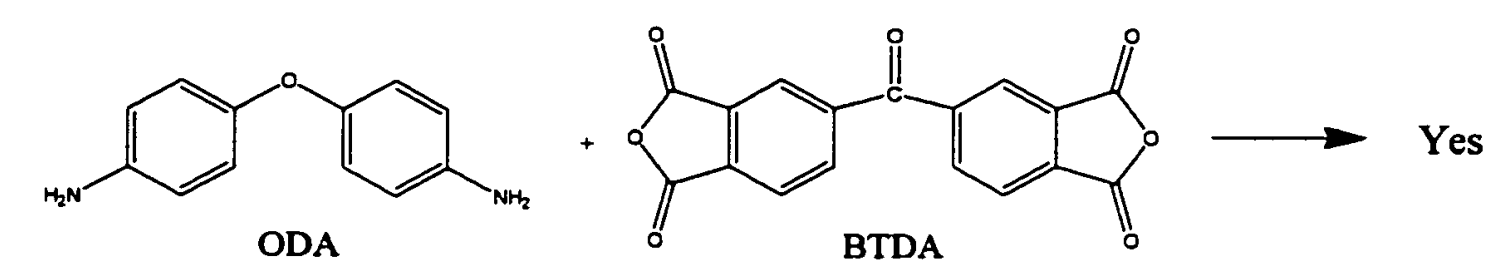<smiles>CC(C)CC(C)C</smiles>

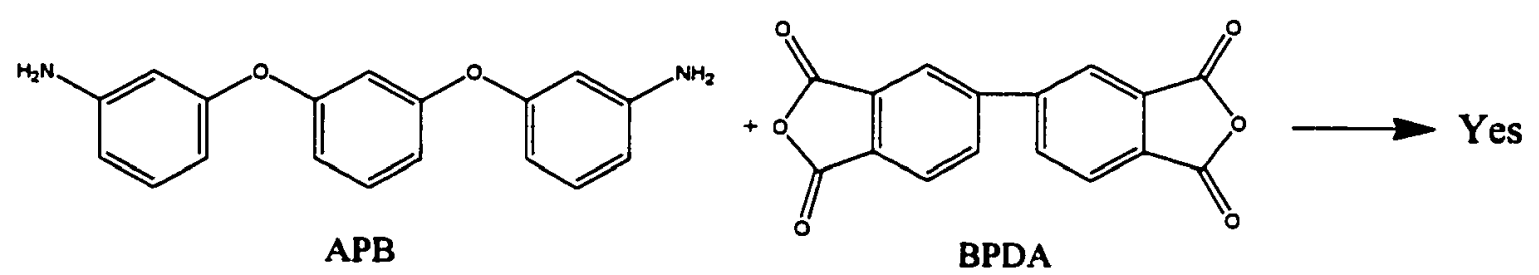

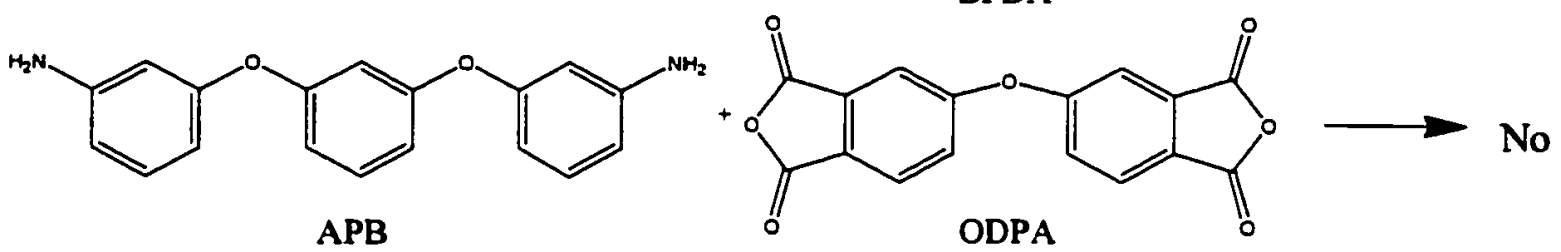<smiles>Cc1ccc(Br)cc1</smiles><smiles>Nc1ccc(C(=O)c2ccc(N)cc2)cc1</smiles>

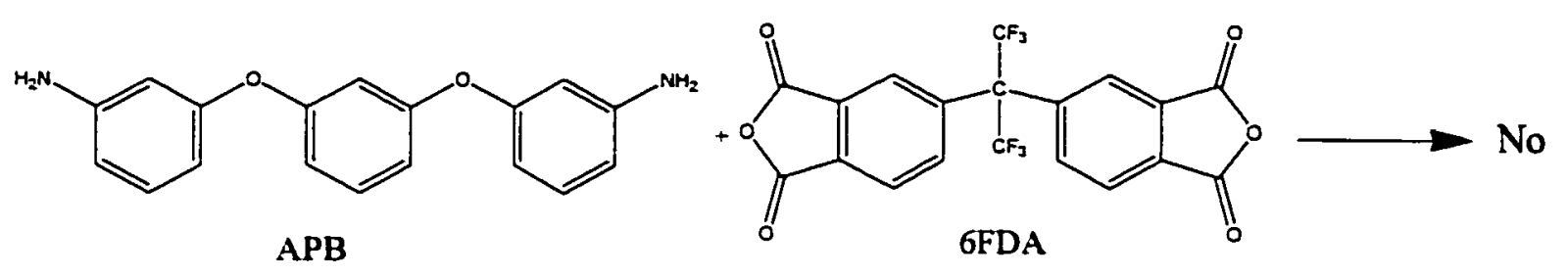


<smiles>Nc1cccc(Oc2cc(Oc3cccc(N)c3)cc(P(=O)(c3ccccc3)c3ccccc3)c2)c1</smiles><smiles>CC(C)C(=O)OC(=O)c1ccc(-c2ccc3c(c2)C(=O)OC3=O)cc1</smiles>

3-APPO

BPDA<smiles>Nc1cccc(Oc2cc(Oc3cccc(N)c3)cc(P(=O)(c3ccccc3)c3ccccc3)c2)c1</smiles>
3-APPO<smiles>N#[W]=C1OC(=O)c2cc(Oc3ccc4c(c3)C(=O)OC4=O)ccc21</smiles><smiles>Nc1ccc(S(=O)(=O)c2ccc(N)cc2)cc1</smiles>

DDS<smiles>Cc1ccc([N+](=O)[O-])cc1</smiles>

BTDA

Table 1. Effectiveness of SCPX-2003 in polyimide nanocomposites

In Table 1 are listed the reactions atempted to prepare clear PAA/SCPX-2003 systems via in-situ polymerization. The mixtures that were not visually clear are marked "No" in Table 1 and no further characterization was performed.

It is not completely clear why some systems are compatible while others are not. If the monomers do not intercalate into the gallery, then the polymerization occurs outside of the clay galleries and there is no exfoliation. One of the major problems in hybrid formation is the small driving force for the monomers to enter the gallery. Among the factors affecting monomer intercalation are polarity, size, and flexibility. It is outside the scope of this dissertation to determine the effects of these factors. However, there are 
some correlations between the effectiveness of different monomer combinations and the molecular structures of the monomers.

Unfavorable clay dispersions resulted with highly polar diamines including those containing carbonyl, sulfone, or phospine oxide groups present on the diamine. One example is the carbonyl-containing BPD-BTDA system, which did not form exfoliated clays when prepared in the presence of SCPX-2003. When the carbonyl on the BPD is replaced with oxygen (ODA), the reaction yields well dispersed nanocomposites. On the other hand, when the carbonyl is replaced with the sulfone group (DDS), there is no dispersion. These comparisons suggest that the polarity of the BPD and DDS monomers operates against intercalation into the clay inner layer. The same effect is seen in the reaction of 3-APPO and BPDA. This in-situ reaction did not form exfoliated nanocomposites but the same procedure with APB, which has no phosphine oxide pendant group, formed exfoliated nanocomposites. This again could be a result of the diamine being restricted from the clay inner layer.

The functional groups present on the dianhydride appear to have a role in exfoliation. Polar substituents in the dianhydride seemed to hinder polymerization in the inner layer. The APB-BPDA system yielded clear mixtures and films but the APB-6FDA system did not. The trifluoroisopropylidene groups present on the 6FDA are suspected to be the reason for this. The trend seen in polarity does not always hold true. For example the ODA-BTDA system yielded clear mixtures and films even though the dianhydride has a carbonyl present. Another surprising reaction was that of APB-ODPA. The ODPA did not have a carbonyl on the monomer but had oxygen instead. This system did not yield exfoliated nanocomposites, which leads one to believe that there are more factors than 
polarity involved. No conclusive statements can be made about these systems but the results can be used to compare with other polyimide/organoclay preparations.

3.4. Thermal characterization of selected polyimide/organoclay hybrids prepared via insitu polymerization

The results of dynamic thermogravimetric analyses performed on the films of APB-BPDA and ODA-BTDA containing various amounts of SCPX-2003 clay in air are presented in Figure 4. The temperature for $5 \%$ weight loss in air increased with the addition of the clay.

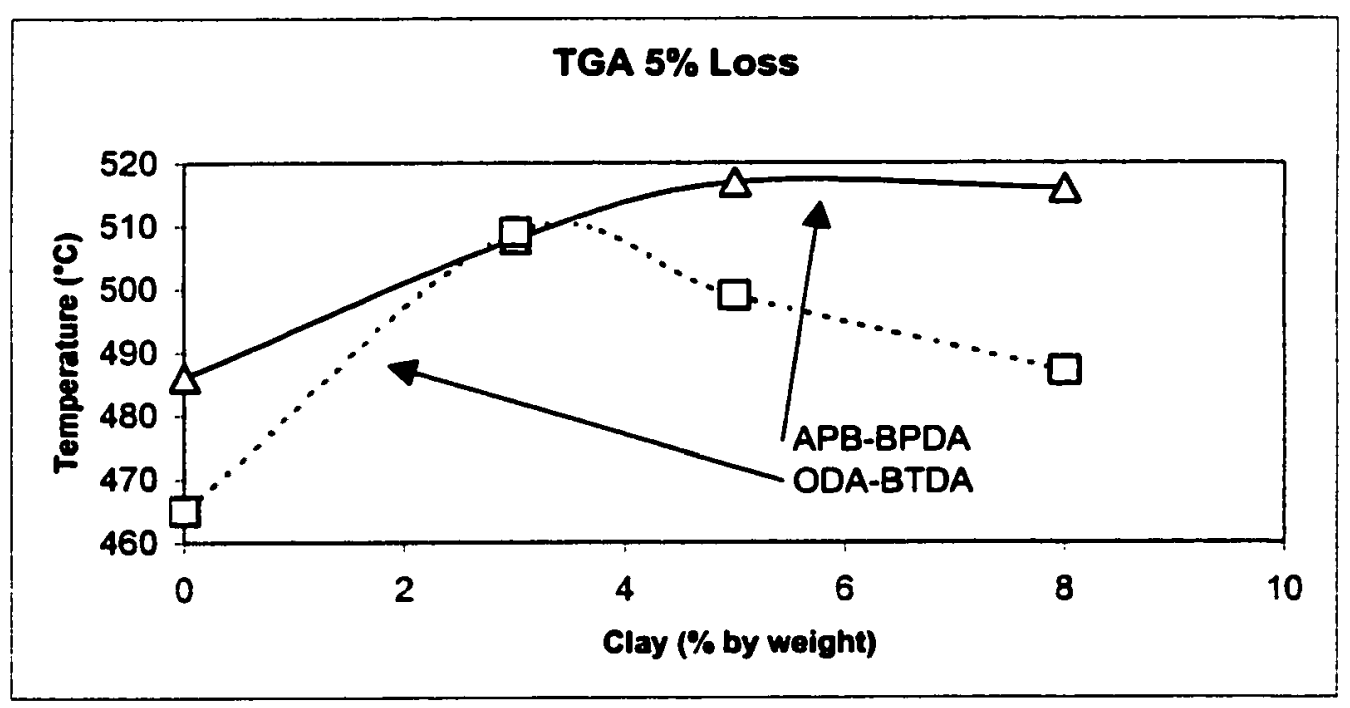

Figure 4. Temperature of $5 \%$ weight loss in air versus clay loading

The TGA curves obtained on the organoclay powder in air and nitrogen is presented in Figure 5. The clay began to lose mass in air at $150^{\circ} \mathrm{C}$, lost approximately $1 \%$ at $200^{\circ} \mathrm{C}$, $5.5 \%$ at $250{ }^{\circ} \mathrm{C}$, and $12 \%$ at $300^{\circ} \mathrm{C}$. 


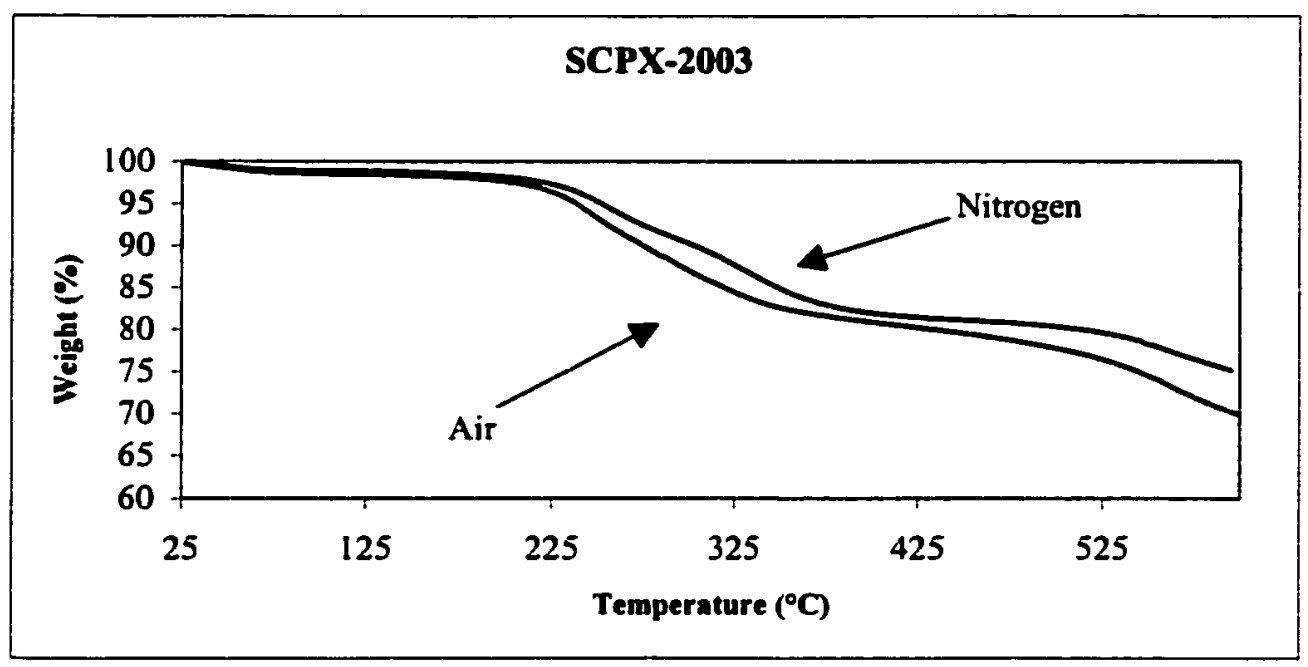

Figure 5. TGA of quaternary tallow ammonium organoclay in air and nitrogen

This is presumably due to the loss of the organic cation and more specifically the degradation of the $18-20$ hydrocarbon tail. The degradation products at $200^{\circ} \mathrm{C}$ have been identified as long hydrocarbon chains, with additional degradation of the long chains as the temperature was increased ${ }^{22}$. The TGA curve obtained on the organoclay powder in nitrogen is also presented in Figure 5. The organoclay is more thermally stable when heated under a nitrogen atmosphere. Weight loss data again shows initial loss at $150^{\circ} \mathrm{C}$, but only a $4 \%$ loss at $250^{\circ} \mathrm{C}$, and $9 \%$ loss at $300^{\circ} \mathrm{C}$.

The white organoclay powder was thermally treated in both air and nitrogen using the same cure cycles as described for the films. After $1 \mathrm{hr}$ at $100^{\circ} \mathrm{C}$, no visible darkening of the clay powder was observed under either atmosphere; however, after $1 \mathrm{hr}$ each at 100 and $200{ }^{\circ} \mathrm{C}$ in air, the clay powder was light tan. Thermal treatment for $1 \mathrm{hr}$ each at 100 and $200{ }^{\circ} \mathrm{C}$ in nitrogen resulted in a slightly off-white powder. After treating the organoclay for $1 \mathrm{hr}$ each at 100,200 and $300^{\circ} \mathrm{C}$ it turned black in air and light tan in nitrogen. Thus the darkening of the films was attributed to the oxidation and degradation 
of the long chain aliphatic quarternary ammonium cation. This is consistent with previous reports regarding the thermal stability of aliphatic quarterary ammonium salt modified clays ${ }^{22}$. From these results on the SCPX-2003 organoclay, it appeared that the organic cation is slightly more stable in nitrogen than in air. This slightly higher thermal stability may account for the different color obtained for the $3 \%$ clay containing APBBPDA films cured under nitrogen as compared to those thermally treated in air.

The degradation of the organic cation at polyimide-curing temperatures poses concern to the preparation of polyimide/clay nanocomposites via thermal imidization. Alternative approaches involving room-temperature catalyzed cyclodehydration and thermal imidization in solution are currently under investigation. Solution imidization can be accomplished at temperatures below $200{ }^{\circ} \mathrm{C}$ with an appropriate solvent and azeotroping agent. However, this method is limited to soluble polyimides.

Both APB-BPDA and ODA-BTDA polyimides are amorphous in their unmodified forms as evidenced by DSC and XRD analyses. DSC was performed on thin film samples. The $\mathrm{Tg}$ for the neat APB-BPDA polyimide film was found to be $20{ }^{\circ} \mathrm{C}$ and was constant within $1{ }^{\circ} \mathrm{C}$ for the hybrid films with up to $8 \%$ clay. The $\mathrm{Tg}$ for the neat ODA-BTDA system was $283^{\circ} \mathrm{C}$ and remained constant within $4^{\circ} \mathrm{C}$ for the hybrid films containing $0-8 \%$ clay.

3.5. TEM analysis of clay dispersion in hybrid films prepared via in-situ polymerization

TEM was performed on thin, cured 3\% clay APB-BPDA polyimide films prepared via in-situ polymerization. Large areas of the films were examined in order to 
obtain a representative analysis of the degree of exfoliation. The results indicated that a high degree of exfoliation of clay nanometer sized particles was obtained. Representative micrographs of different sites on the same polyimide/clay film are presented in Figures 6 and 7. Although good dispersion was achieved as seen in Figure 6, Figure 7 shows larger and thicker clay particles. In the latter case, clay particles have apparently formed agglomerates in some areas of the film during thermal treatment in air. Figure 7 shows some clay particles that have collapsed. Presumably, some clay particles were not fully wetted by the polymer in these regions and upon thermal degradation of the organic cation, the galleries collapsed. The percentage of dispersion cannot be quantified by examination of these micrographs but it appears from Figure 8, in which the magnification has been reduced, that exfoliation dominates. These exfoliated particles are $\sim 200$ to $700 \mathrm{~nm}$ in length and $\sim 1-10 \mathrm{~nm}$ thick.

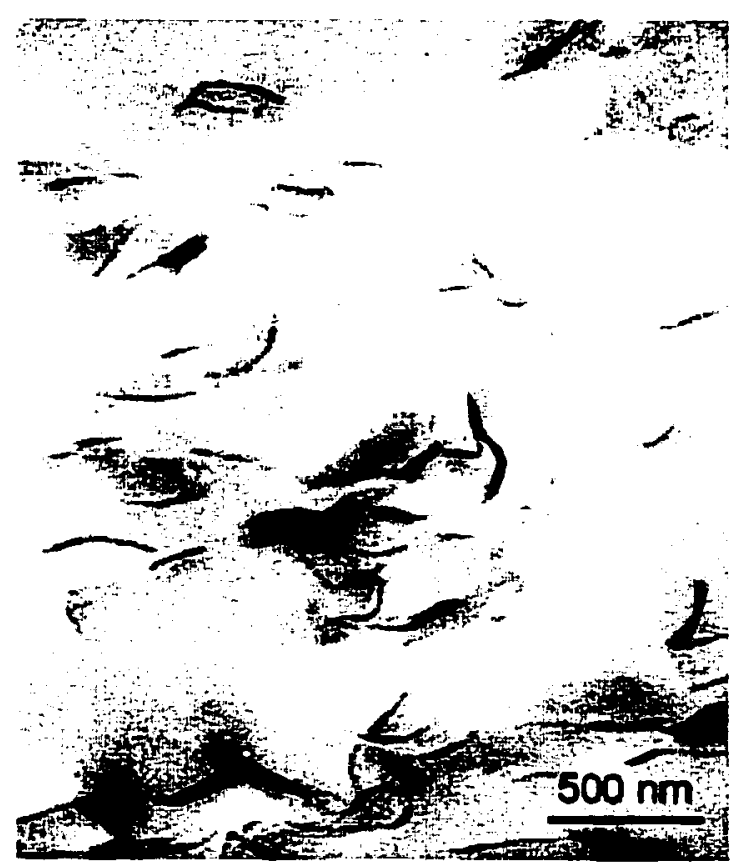

Figure 6. TEM micrograph of $3 \%$ clay APB-BPDA films showing exfoliated clays 


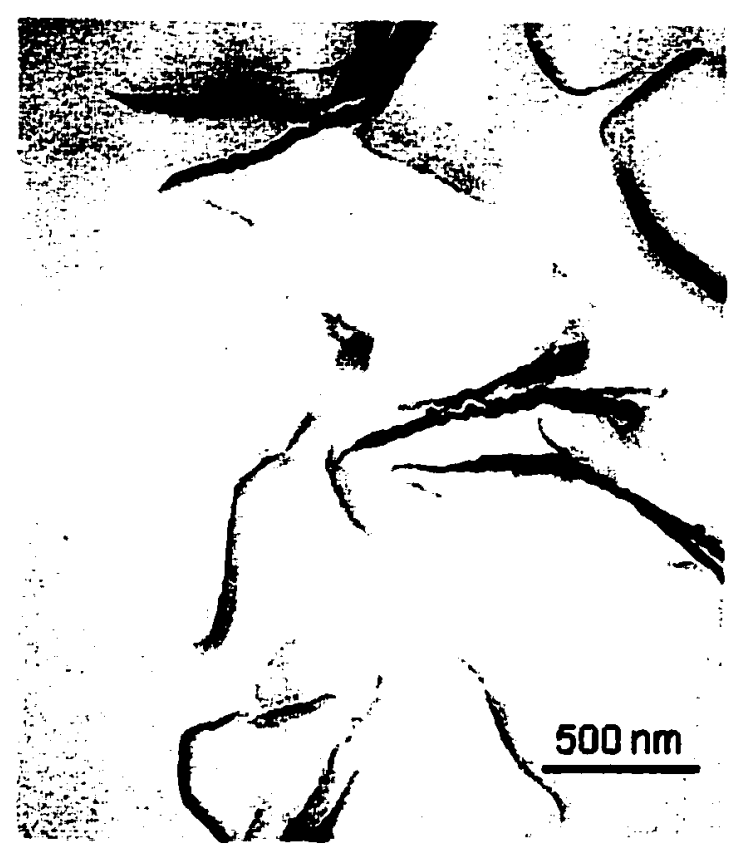

Figure 7. TEM micrograph of $3 \%$ clay APB-BPDA films showing some collapsed clay

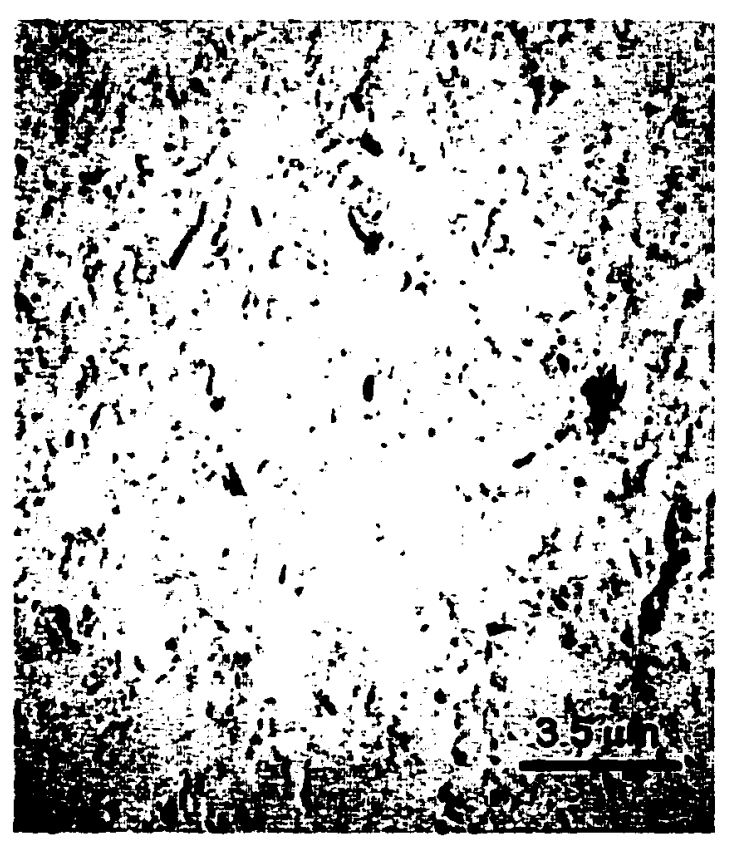

Figure 8. Reduced magnification micrograph of $3 \%$ clay APB-BPDA films 
3.6. XRD analysis of clay samples and hybrid films prepared via in-situ polymerization

Although visually transparent films were obtained through the in-situ polymerization method, both TEM and XRD indicated agglomerates in the thermally imidized films. The XRD data of thermally imidized films cured to $300^{\circ} \mathrm{C}$ in air

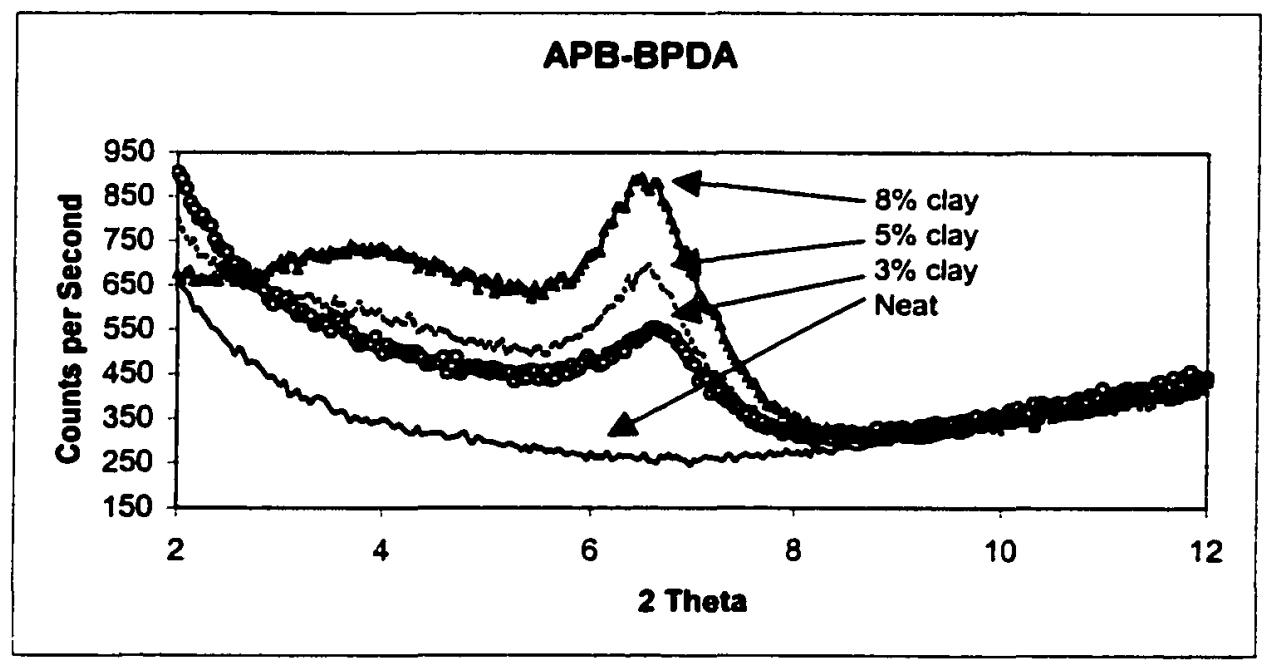

Figure 9. XRD patterns of air-cured APB-BPDA thin film samples

prepared from APB-BPDA corresponding to $0,3,5$, and $8 \%$ clay loadings are presented in Figure 9. The peak at $2 \theta=6.6^{\circ}$ for the $3 \%$ sample corresponds to a d-spacing of $\sim 1.34$ nm for the 001 reflection. The 5 and $8 \%$ peaks shift slightly so that in the $8 \%$ sample the $\mathrm{d}$-spacing is $\sim 1.36 \mathrm{~nm}$. A broad peak centered near $2 \theta=4^{\circ}$ for the $8 \%$ clay sample may be the consequence of small, residual amounts of the SCPX-2003 clay whose galleries were not penetrated by monomer and did not collapse (see Figurel1). Analogous data for thermally imidized hybrid films prepared from ODA-BTDA and cured to $300{ }^{\circ} \mathrm{C}$ in air are presented in Figure 10. The peak centered at $2 \theta=4^{\circ}$ appears again in the $8 \%$ clay sample while all the samples have a peak near $2 \theta=6.6^{\circ}$. These results are similar to the 


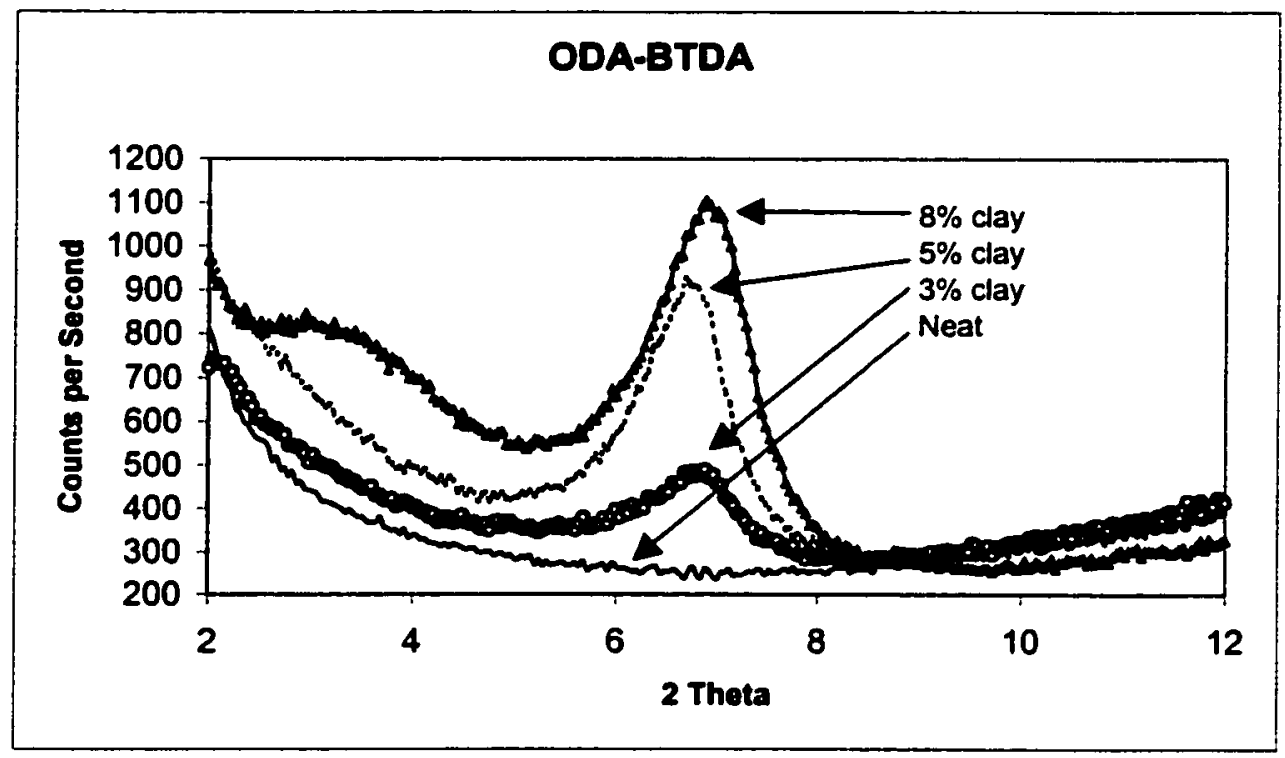

Figure 10. XRD patterns of air cured ODA-BTDA thin film samples

APB-BPDA sample but show a slightly smaller d-spacing $(1.28 \mathrm{~nm})$. This peak indicates the presence of some clay particles close to or even smaller than sodium montmorillonite. In the XRD data in two previous publications, both of which concern polyimides, small peaks appear with similar $2 \theta$ values ${ }^{17,24}$. XRD patterns of the organoclay and of sodium montmorillonite are shown in Figure 11. By XRD, the sodium clay has a d-spacing of

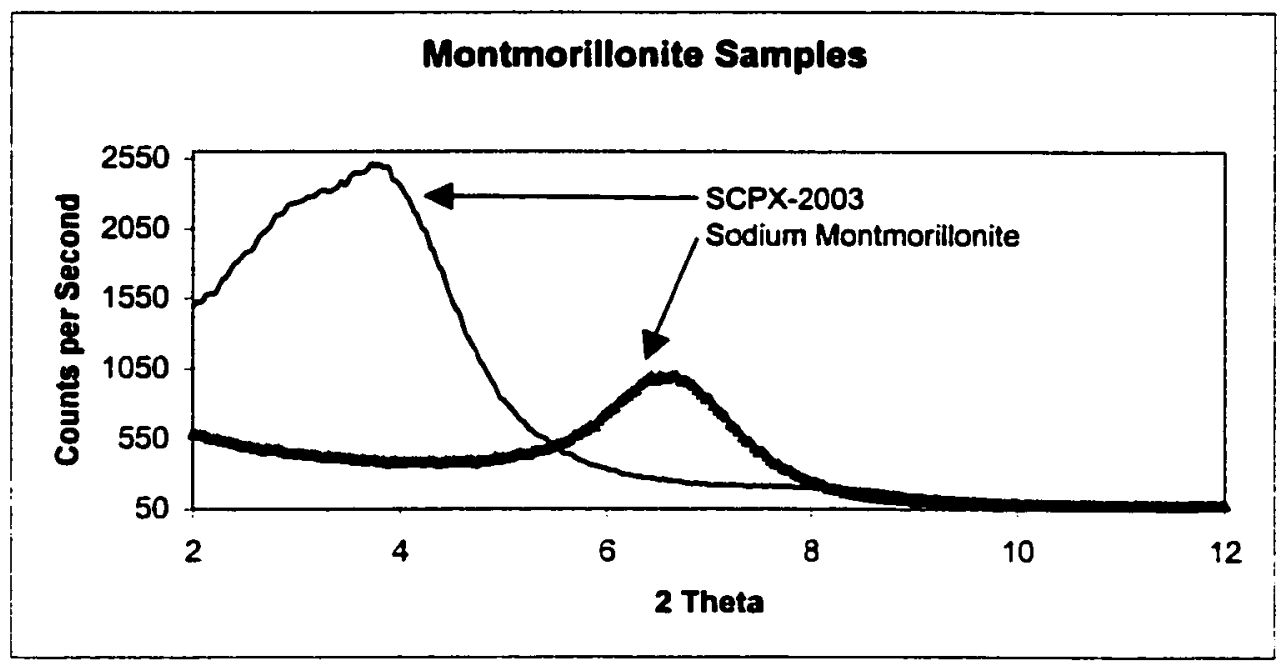

Figure 11. XRD patterns of sodium montmorrillonite and organoclay (SCPX-2003) 


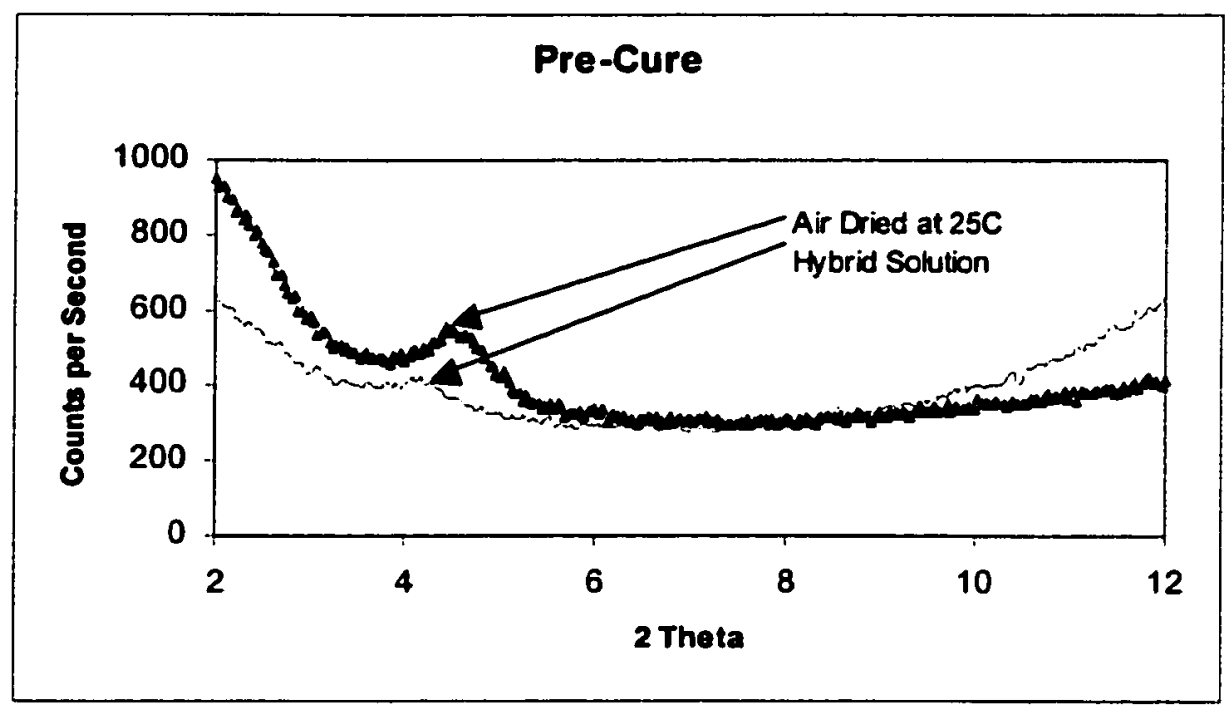

Figure 12. XRD patterns of 3\% clay APB-BPDA poly(amide acid) hybrid mixture and film after air drying at room temperature clay agglomerates that apparently retained their original structure. It is doubtful that they were impregnated by monomer prior to polymerization. XRD data (Figure 13), collected after each step in the cure cycle in air $\left(100,200,300^{\circ} \mathrm{C}\right)$ shows the loss of the $2 \theta=4.1$ and $4.3^{\circ}$ peaks and the formation of a new peak at $2 \theta=6.6^{\circ}(1.34 \mathrm{~nm})$. This new peak increased in intensity as the temperature was increased. Thus, it was apparent that the thermal decomposition of the organocation resulted in a collapse of the clay layers. 


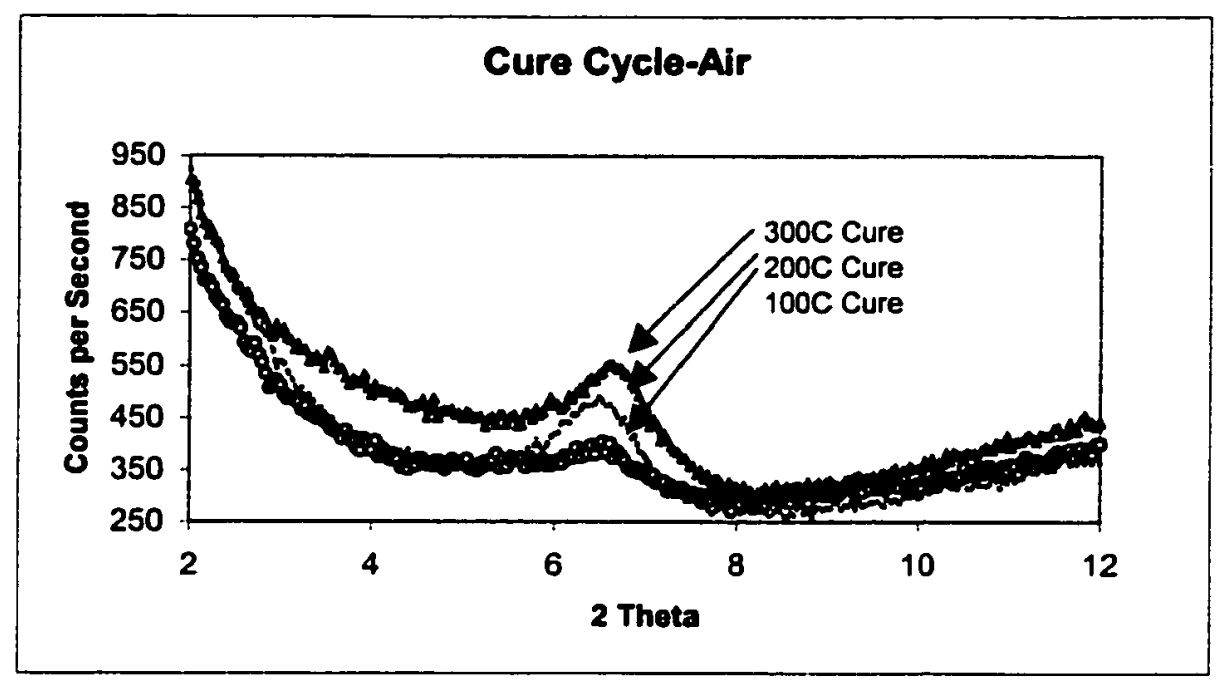

Figure 13. XRD patterns of $3 \%$ clay APB-BPDA films treated at different temperatures in air.

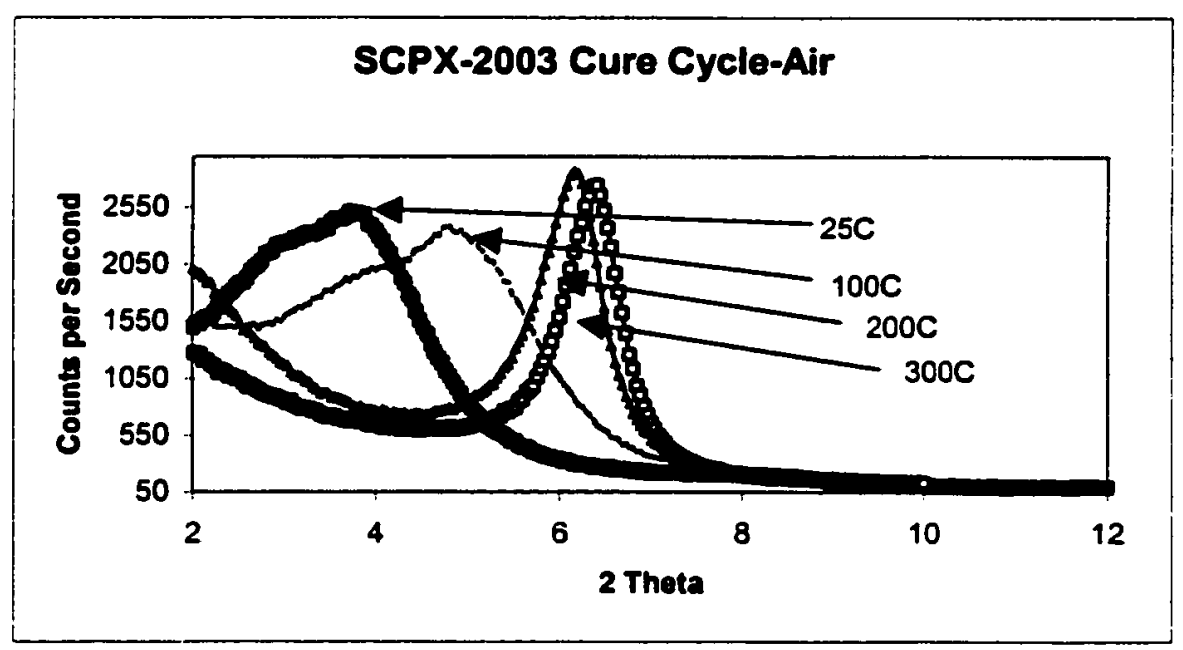

Figure 14. XRD patterns of organoclay treated at different temperatures in air

To further test the hypothesis that the organoclay degrades on heating, the neat clay was subjected to the same thermal treatment as that used for the hybrid films. The XRD patterns of the clays showed the 001 peak shifting to larger $2 \theta$ values upon heating.

A similar analysis was performed on films that were thermally treated in nitrogen instead of air. 3\% APB-BPDA poly(amide acid) and polyimide films prepared via in-situ 
polymerization and dried in the same manner as the films discussed above showed a delayed formation of the collapsed layer agglomerates. As illustrated in Figure 15, the 001 peak remained at $2 \theta=4.3^{\circ}(2.05 \mathrm{~nm})$ in the sample held at $100^{\circ} \mathrm{C}$ for $1 \mathrm{hr}$. After

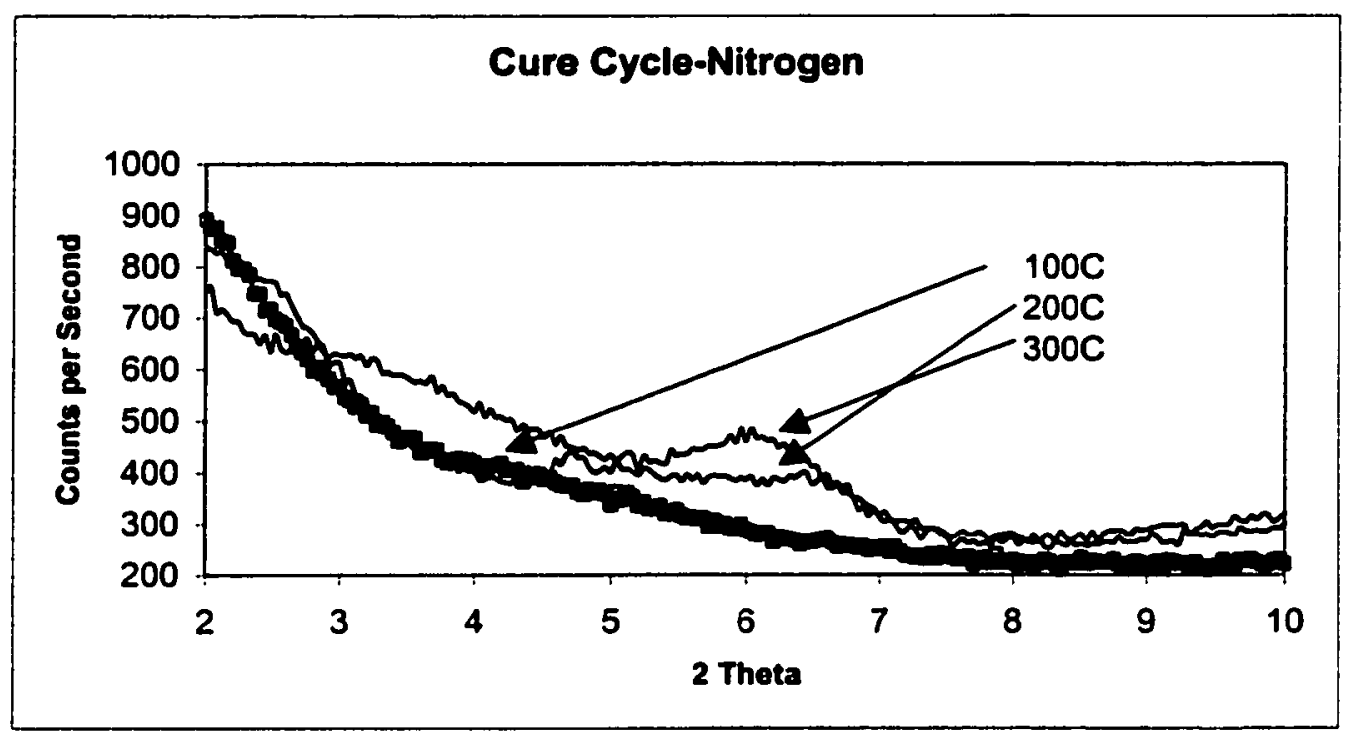

Figure 15. XRD patterns of $3 \%$ clay APB-BPDA films treated at different temperatures in nitrogen

holding a film for $1 \mathrm{hr}$ each at $100^{\circ} \mathrm{C}$ and $200^{\circ} \mathrm{C}$, the XRD pattern indicated that there was layer spacing that varied from $2 \theta=4.3^{\circ}(2.05 \mathrm{~nm})$ to approximately $2 \theta=6.0^{\circ}(1.47$ $\mathrm{nm}$ ) for the collapsing clay particles. After holding for $1 \mathrm{hr}$ each at 100,200 , and $300^{\circ} \mathrm{C}$ the XRD peak was more defined and had a maximum at approximately $2 \theta=6.0^{\circ}(1.47$ $\mathrm{nm})$. Figure 16 shows that this same trend was followed in the organoclay as it was subjected to the same thermal conditions. The organoclay sample that was subjected to a $1 \mathrm{hr}$ hold each at $100^{\circ} \mathrm{C}$ and $200^{\circ} \mathrm{C}$ in nitrogen exhibited a 


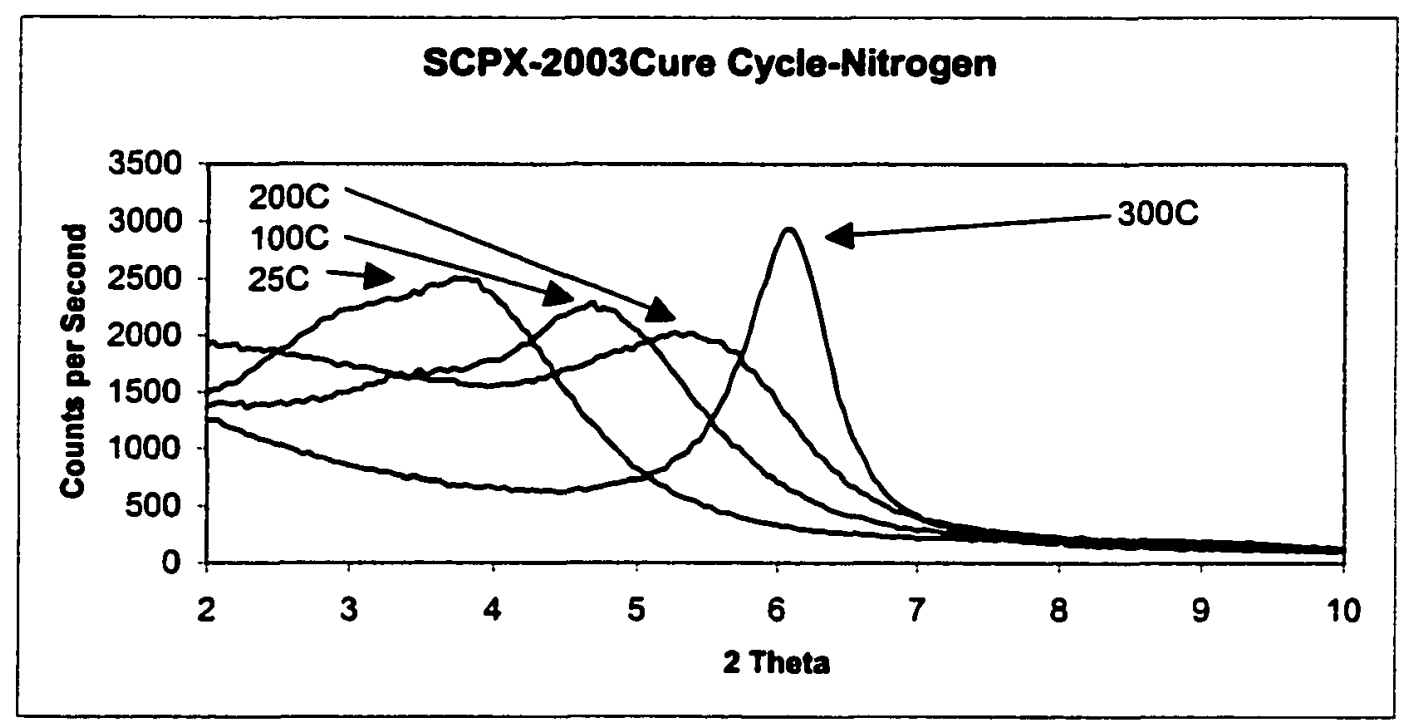

Figure 16. XRD patterns of organoclay treated at different temperatures in nitrogen maximum at $2 \theta=5.2^{\circ}(1.70 \mathrm{~nm})$ indicating that the interlayer cation did not completely degrade at this temperature. The peak for the organoclay sample held for $1 \mathrm{hr}$ each at 100,200 , and $300^{\circ} \mathrm{C}$ under nitrogen was centered at approximately $2 \theta=6.0^{\circ}(1.47 \mathrm{~nm})$ which is similar to the peak in the film sample treated in nitrogen but is at a slightly lower value than the samples that were treated at these temperatures in air. The organoclay did not appear to collapse in nitrogen as it did in air indicating that a thermoxidative mechanism is more damaging to the interlayer cation than a mechanism involving nitrogen.

3.8. Mechanical properties of hybrid films prepared by in-situ polymerization

In Figure 17, the tensile modulus of thin films after imidization in air is plotted against clay concentration. A monotonic increase in modulus was observed as the clay concentration was increased from a 0 to $8 \%$ loading for both the APB-BPDA and the 
ODA-BTDA systems. This was as expected since the clay particles inherently possess high moduli and if dispersed on the nanoscale level, should also increase the moduli of polymeric systems. This result is typical of polymeric systems in which nanoscale dispersion of the clay is attained. However, Agag et al. found that the modulus increased up to $2 \%$ clay loading and that further additions of clay lowered moduli. The polyimides used in Agag's work had a more rigid backbone and some molecular ordering ${ }^{24}$.

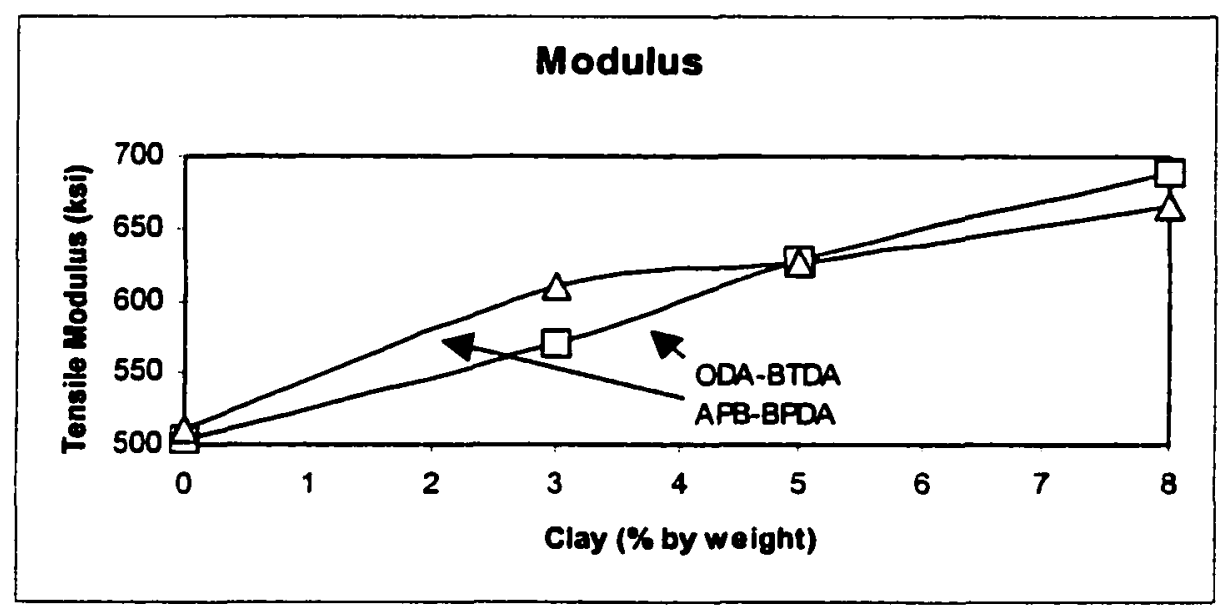

Figure 17. Tensile modulus versus clay loading of air cured polyimide/organoclay systems

Figurel 8 shows a decrease in percent elongation at break with the addition of organoclay. This may be a consequence of the collapsed clay layer agglomerates present. Decreases in tensile strength shown in Figure 18 and a corresponding decrease in elongation to break were observed in both systems, and could be attributed to the same reason. It seems likely that only a small amount of the undispersed organoclay on the nanoscale level would create the effects that were observed for elongation and strength. 
On the other hand it would take a much larger dispersed amount to create the rise in tensile modulus seen in both systems.

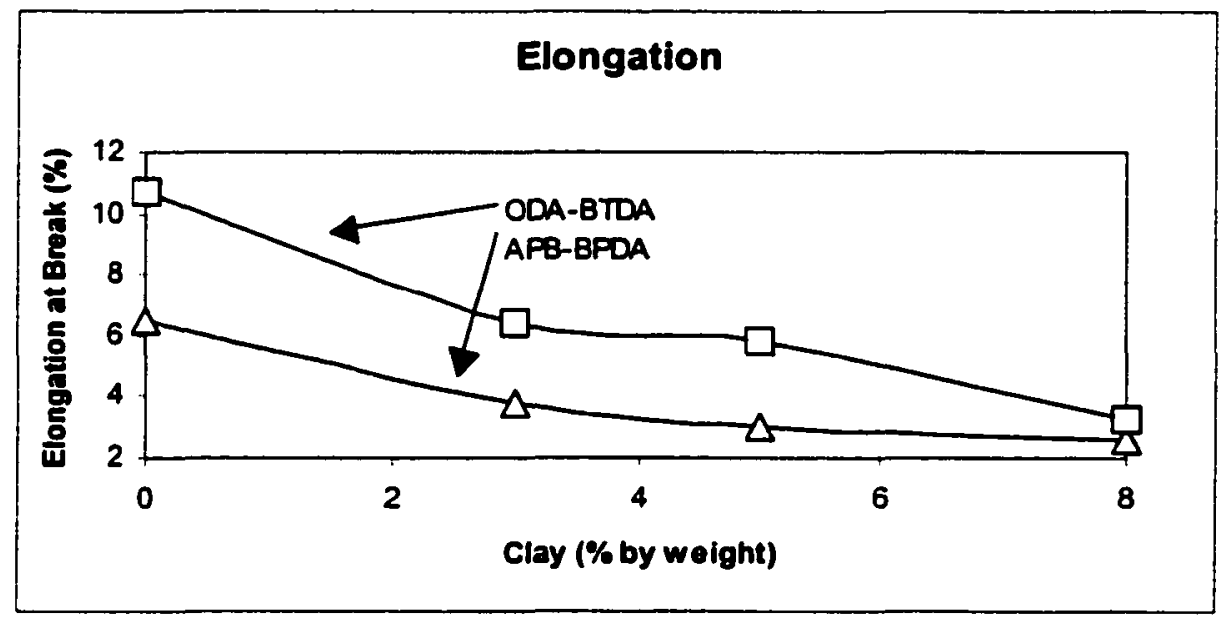

Figure 18. Elongation at break versus clay loading of air cured polyimide/organoclay systems

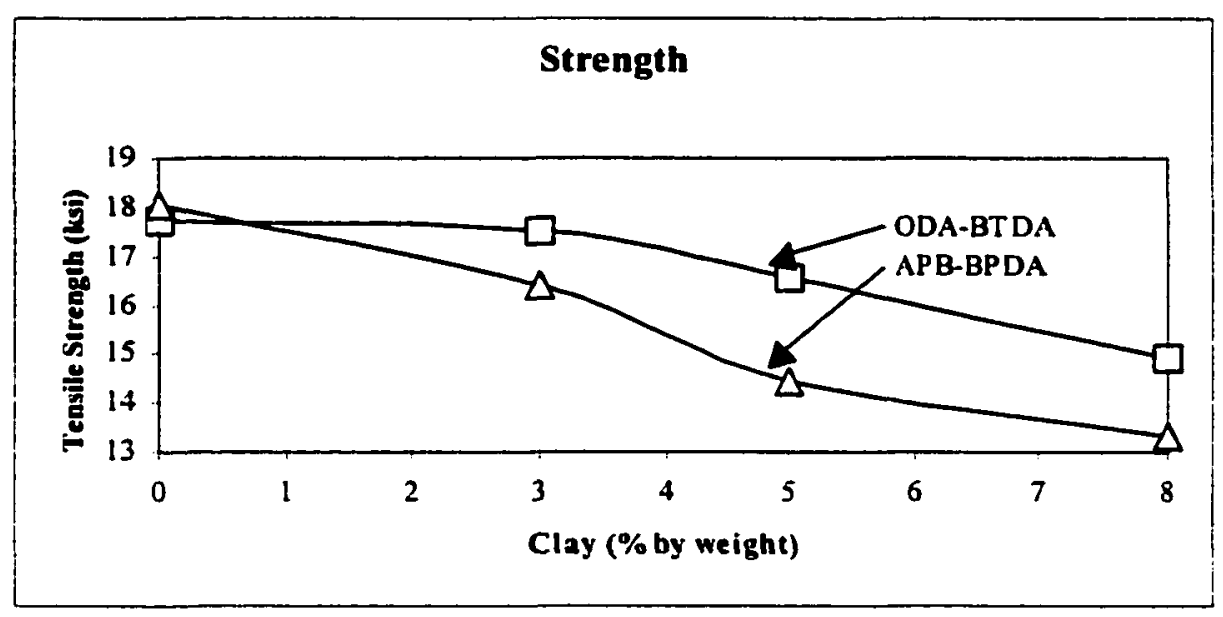

Figure 19. Yield Strength versus clay loading of air cured polyimide/organoclay systems 


\subsection{Characterization of DTMA organoclay/APB-BPDA hybrids}

All of the results up to this point were obtained from nanocomposite materials prepared with SCPX-2003 organoclay. The research continued with other low temperature organoclays. A powdered organoclay prepared from dodecyl trimethyl ammonium bromide and Cloisite- $\mathrm{Na}+$ (Section 2.2) was characterized with XRD. The pattern is below in Figure 20.

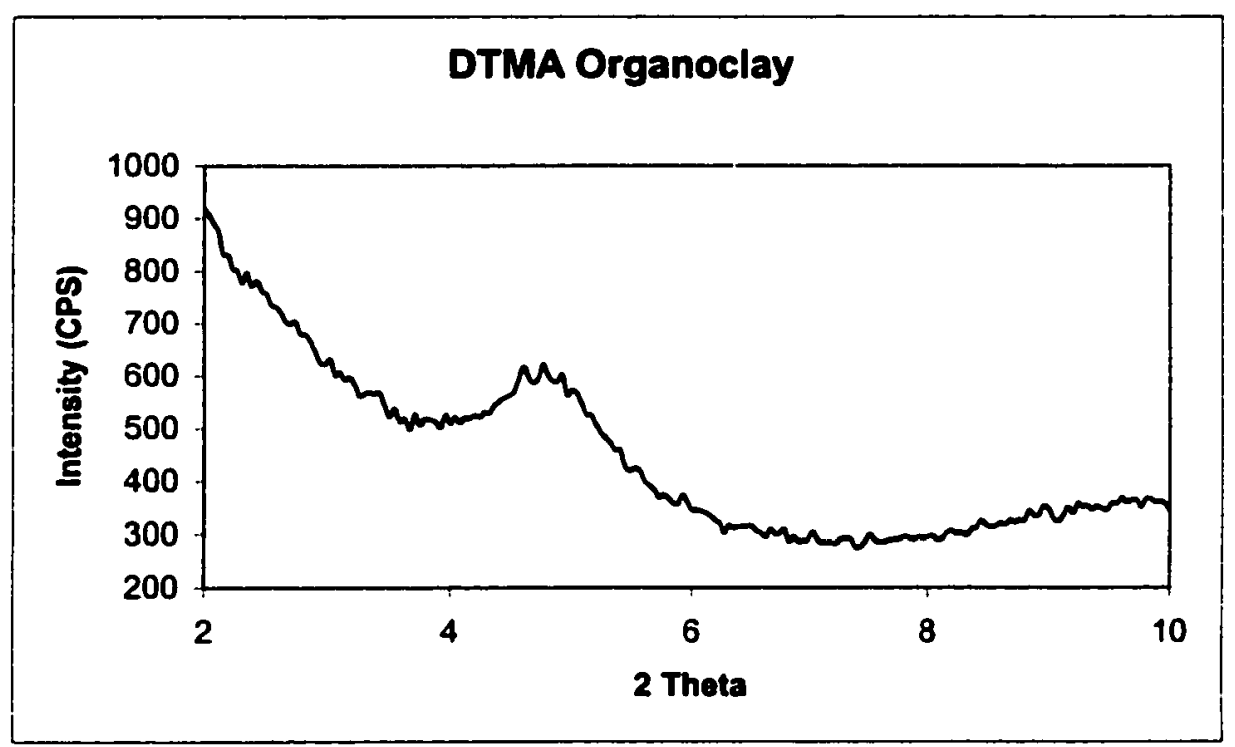

Figure 20. XRD pattern of DTMA organoclay

The d-spacing of this clay was $1.86 \mathrm{~nm}$, which was smaller than that of the SCPX2003, the difference being attributed to the smaller number of carbon atoms in the surfactant tail. High molecular weight APB-BPDA PAA was prepared in the presence of DTMA oranoclays that had been dispersed in organic solvent. The amount of clay relative to the amount of polymer was $3 \%$ by weight. The mixture was yellow and clear after polymerization and stirring. The clarity indicated that the clay had dispersed to nanometer dimensions. This mixture was used to prepare a hybrid film which was clear 
after thermal cure to $300^{\circ} \mathrm{C}$. The film was characterized via XRD and the pattern is shown below in Figure 21.

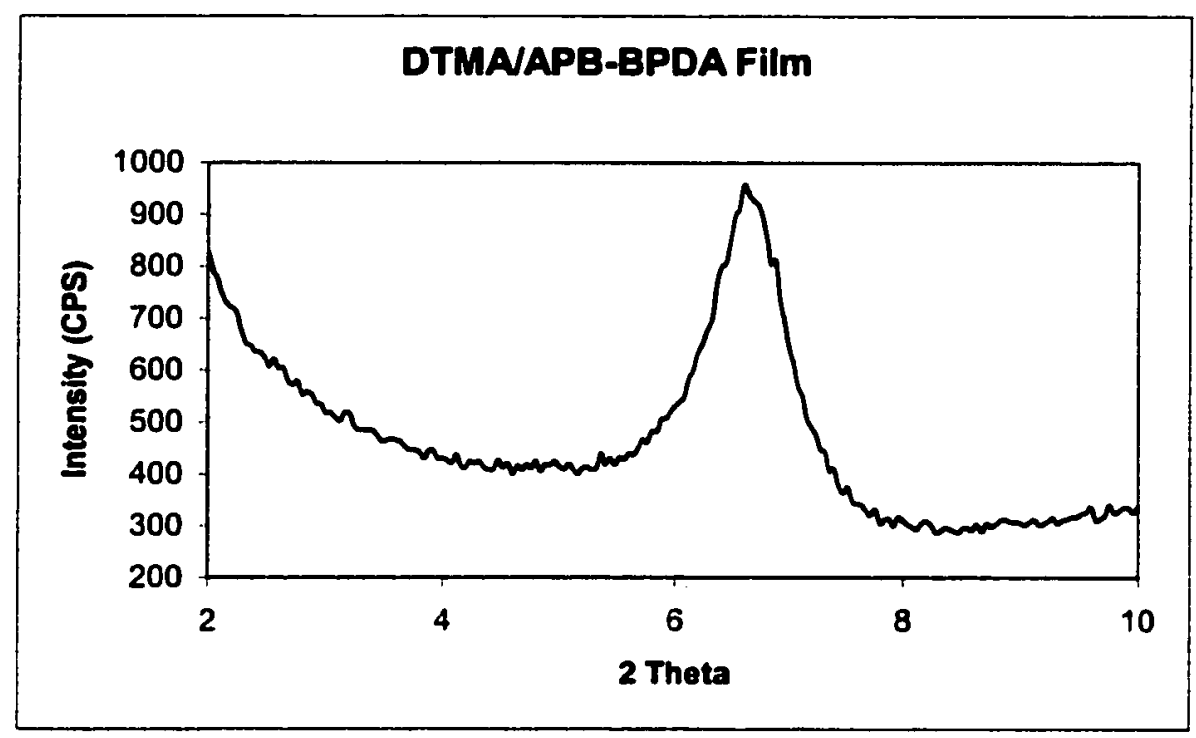

Figure 21. XRD pattern of DTMA/APB-BPDA nanocomposite film

The pattern shown here indicates that there was some clay that had not exfoliated and the clay had collapsed to a smaller d-spacing than the organoclay by itself. This again was indicative of the degradation of the surfactant ion. Some organoclay was found with this d-spacing but with XRD the quantity cannot be determined. Visual inspection of the film indicated that the amount of agglomerates was probably low and similar to the amount in the SCPX-2003/APB-BPDA nanocomposites. No further characterization was performed on these films.

\subsection{Characterization of DTPP organoclay'APB-BPDA hybrids}

Dodecyl triphenyl phosphonium (DTPP) organoclay was prepared as a white powder as described earlier (Section 2.3). The powder was examined with XRD and the results are shown below in Figure 22. 


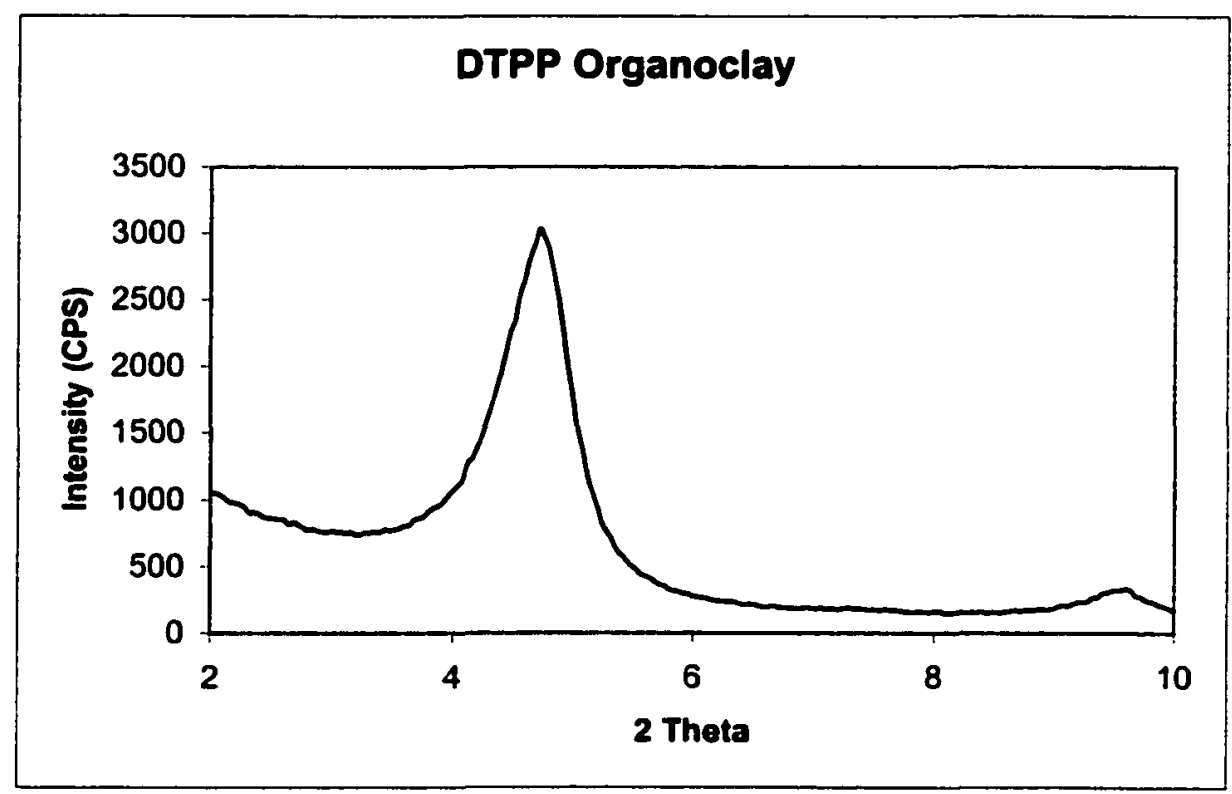

Figure 22. XRD pattern of DTPP organoclay

The peak in this XRD pattern corresponded to the same d-spacing as in the case of the DTMA organoclay, probably a consequence the fact that the carbon tail on the two surfactants are the same length. This organoclay was used in the preparation of nanocomposite materials. High molecular weight PAA of APB-BPDA was prepared in the presence of this clay yielding clear yellow mixtures. A clear film was prepared from this mixture. No further characterization was performed.

\subsection{Characterization of Triton organclays/APB-BPDA hybrids}

Oganoclays prepared with various cations were used in in-situ polymerizations of APB-BPDA poly(amide acid) mixtures and polyimide films. Three different experimental organoclays were received from Triton. The mixtures prepared with these three clays were all clear which was indicative of good dispersion in the PAA. All mixtures were yellow-brown and no major differences were seen from mixture to mixture. The films were all fingernail creasable and were also relatively clear. The films 
were darker than the neat resin indicating that there may have been some degradation of the clay in the curing process.

In the first example a montmorillonte modified with a tallow ammonium ion was incorporated into APB-BPDA polyimide. The surfactant ion was probably the same ion that was used in the SCPX-2003 organoclay. The organoclay was examined with XRD prior to mixing with the polymer.

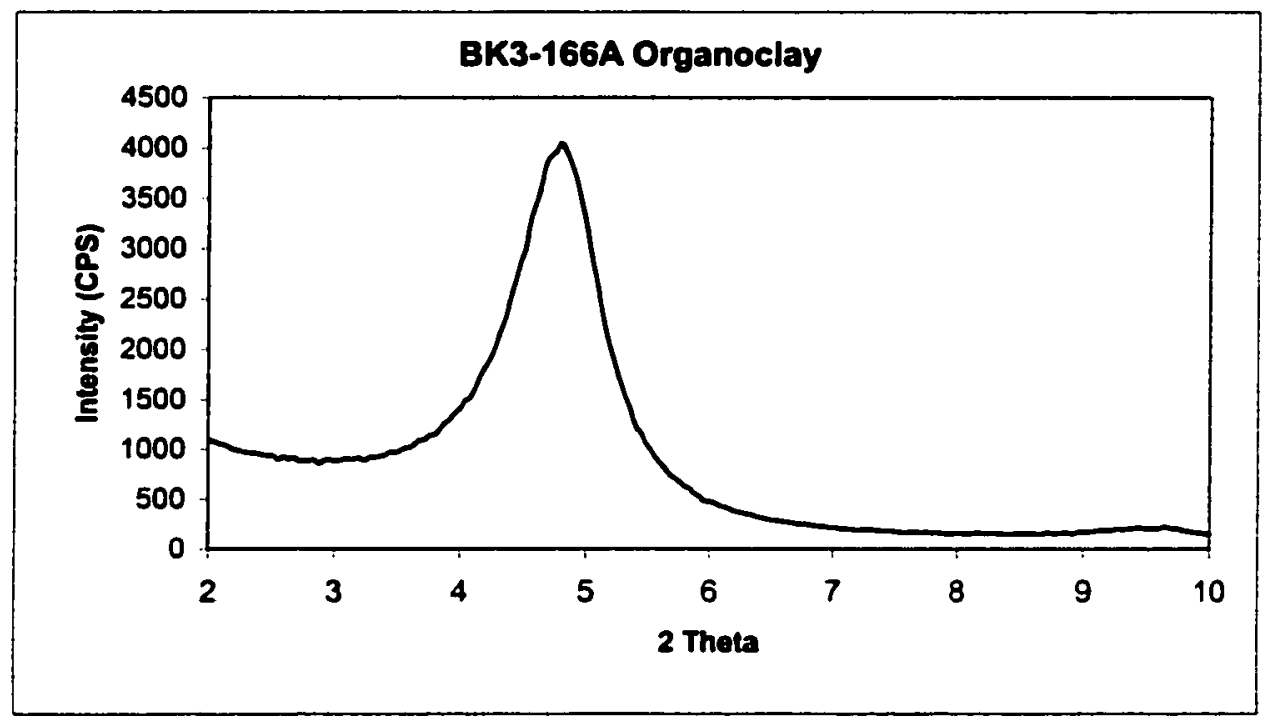

Figure 23. XRD pattern of BK3-166A organoclay

The organoclay had a d-spacing of $1.84 \mathrm{~nm}$ as determined by the peak at $2 \theta=4.8^{\circ}$. This was smaller than the spacing found in the SCPX-2003 $(2.37 \mathrm{~nm})$ and may be a result of the charge on the clay surface. Placing the organoclay in organic solvent and polymerizing APB-BPDA in the presence of the clay resulted in a hybrid mixture. A film was prepared from this hybrid mixture. 


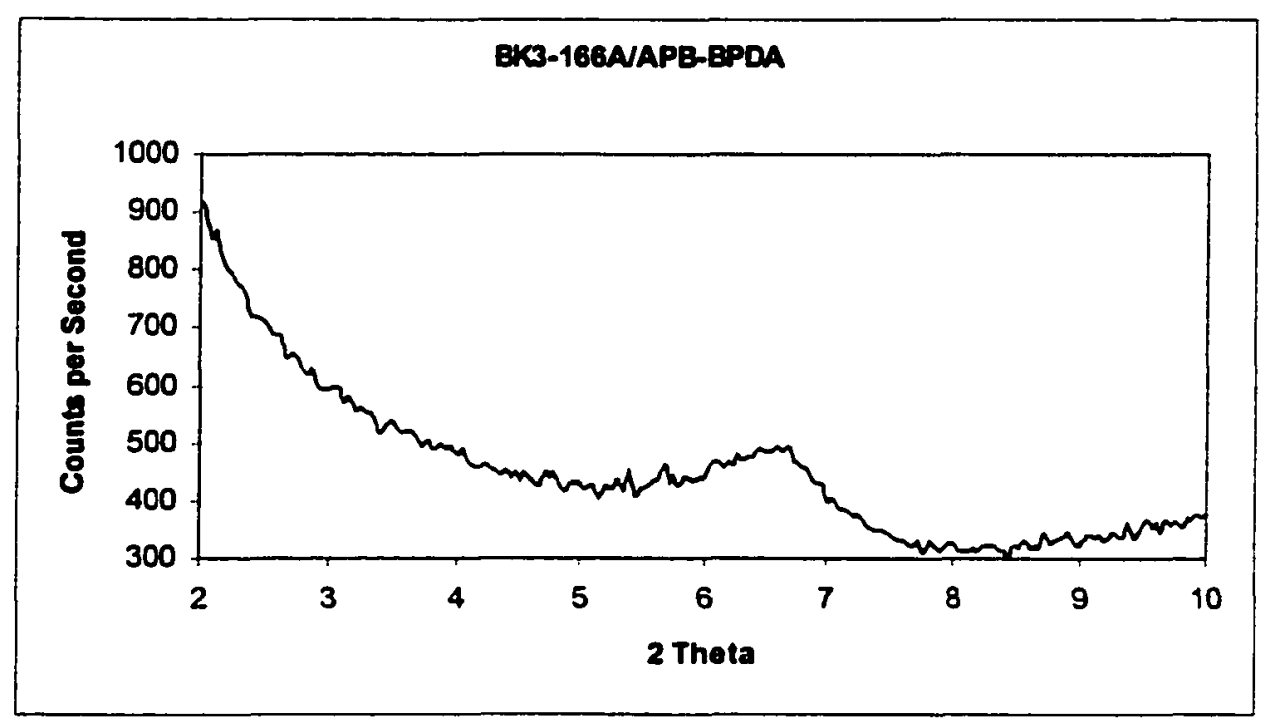

Figure 24. XRD pattern of BK3-166A/APB-BPDA hybrid film

The XRD pattern for this film contained a peak which indicated that the clay was not completely exfoliated. This shift in the peak position to a larger angle showed that the galleries in the clay had partially collapsed after heating. The final layer spacing of the clay was $1.33 \mathrm{~nm}$. This followed the same trend as the SCPX-2003 organoclay and the collapse of the clay layers after heating was most likely a result of cation degradation.

Another organoclay that was received from Triton was used in the preparation of nanocomposite materials. The BK3-166B organoclay had a large d-spacing prior to its use in the polymer. The same surfactant was used to treat the clay as in the previous example but the clay also was treated with an amino silane compound. This was most likely attached via a condensation reaction with any free hydroxyls on the clay surface. 
The XRD pattern in Figure 25 showed that the clay had a d-spacing of $2.23 \mathrm{~nm}$.

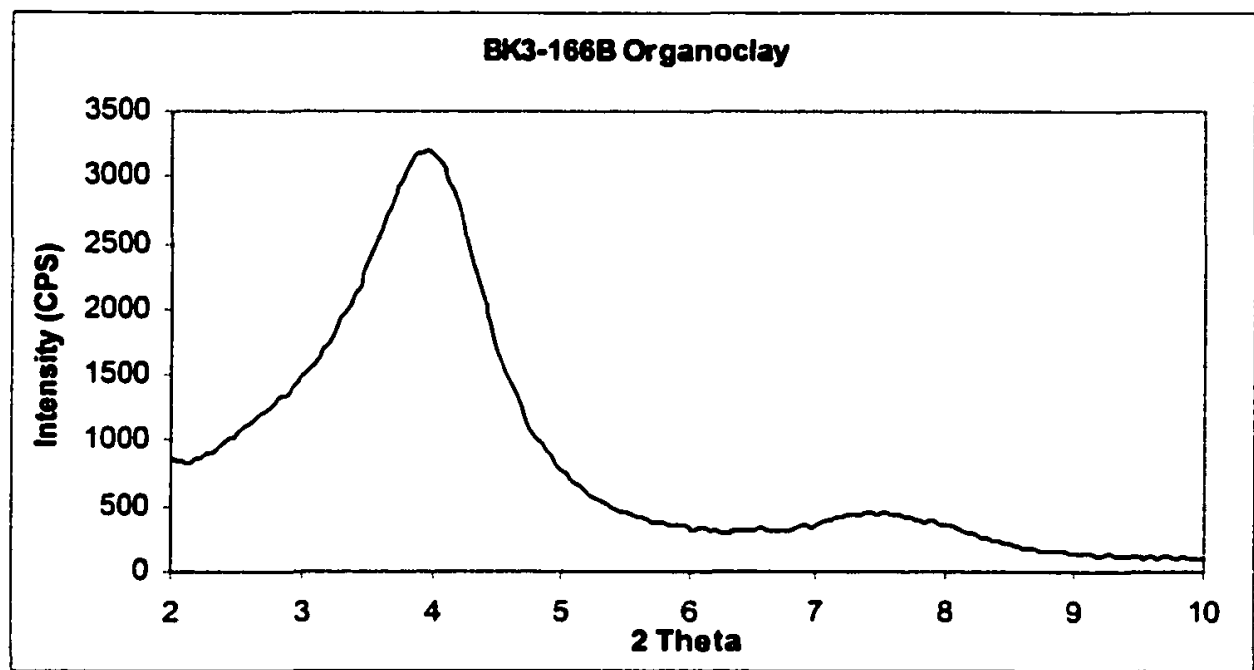

Figure 25. XRD pattern of BK3-166B organoclay

The increase in d-spacing for this clay may be a result of the amino silane compounds on the clay edges as well as any that may have attached to hydroxyls in the clay gallery. This clay was also used in the preparation of APB-BPDA nanocomposites. The nanocomposite film was characterized with XRD and the pattern is shown in Figure 26.

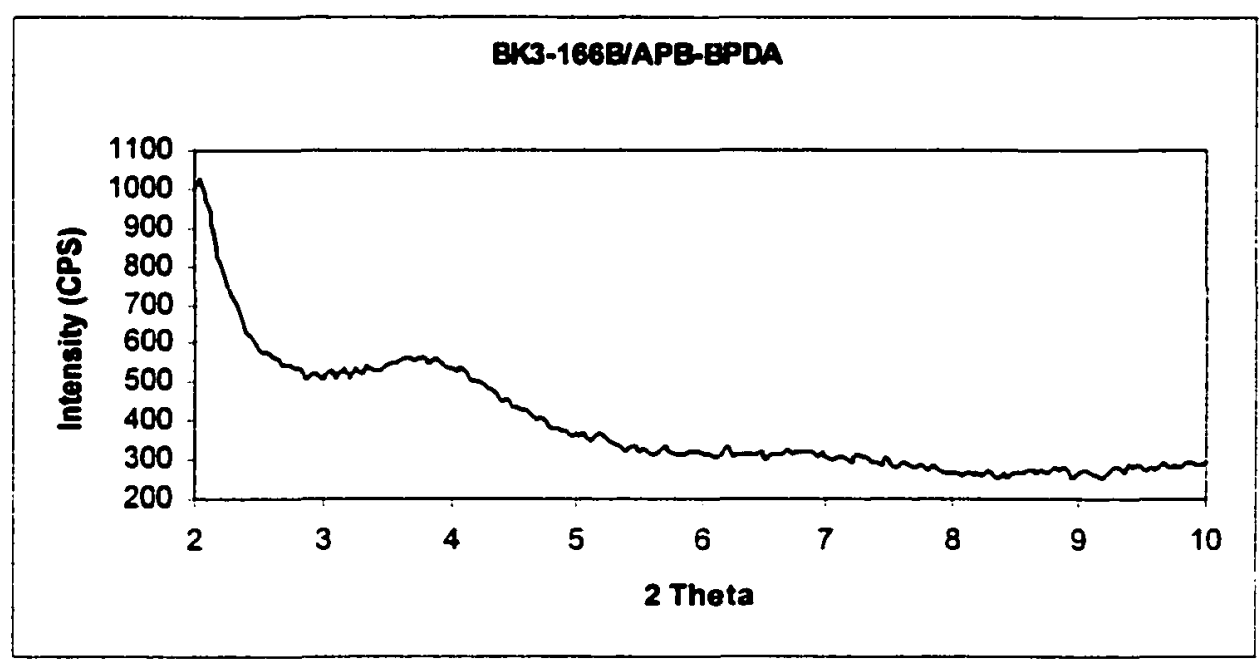

Figure 26. XRD pattern of BK3-166B/APB-BPDA hybrid 
The clay did not completely exfoliate in the film. There is a peak in the XRD pattern at the same position as the peak in the organoclay pattern. The organoclay that did not exfoliate in the polyimide matrix remained at approximately the same d-spacing after curing. This may indicate that the surfactant did not degrade during cure but a better guess would assume that the surfactant did degrade but the amino silane somehow did not allow the clay to collapse. However, this speculation is not supported by any other evidence.

The third organoclay from Triton was the BK3-166C organoclay. This organoclay was modified with a surfactant that had a shorter hydrocarbon chain than the previous two examples. This clay was examined with XRD and the pattern is shown in Figure 27.

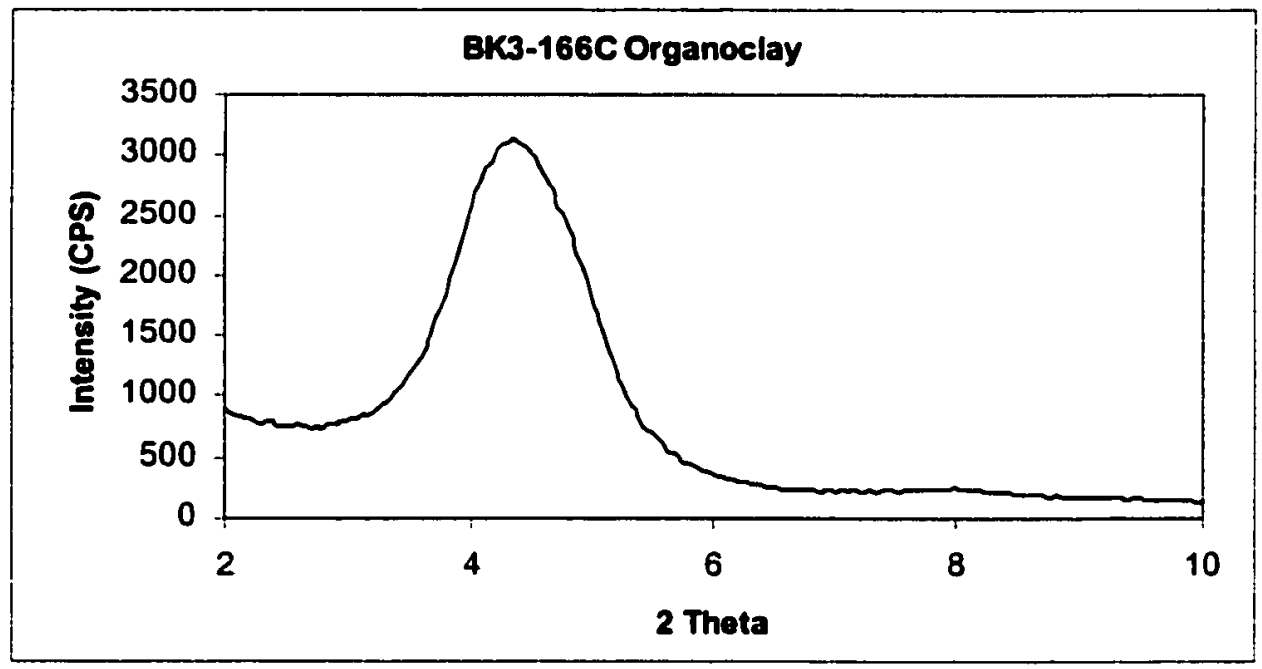

Figure 27. XRD pattern of BK3-166C organoclay

This organoclay had a d-spacing $2.02 \mathrm{~nm}$ prior to hybrid formation. This was a larger dspacing than the organoclays that had the 18 carbon chains. This cannot be explained except that maybe the butyl groups on the phosphonium ion aided in separating the layers. After polymer formation an XRD pattern was obtained of the nanocomposite film and the pattern is shown in Figure 28. 


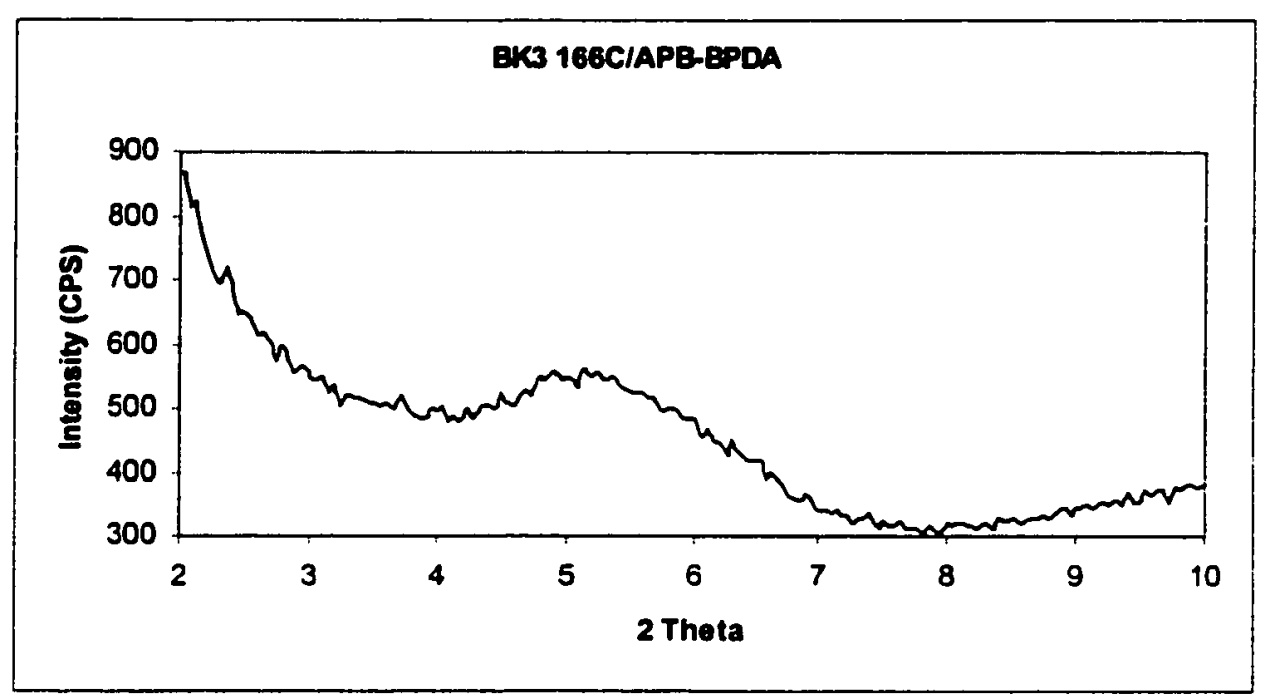

Figure 28. XRD pattern of BK3-166C/APB-BPDA hybrids

The peak in this film indicated that this clay collapsed after in-situ polymerization of APB-BPDA and subsequent curing of the PAA. The peak shifted to $2 \theta=5.16^{\circ}$ after curing. This was again was most likely due to the degradation of the surfactant ion.

The organoclays that were used in this section behaved similarly to the other low temperature organoclays presented in this chapter. The surfactant most likely degraded at the processing temperature $\left(300^{\circ} \mathrm{C}\right)$. The addition of the amino silane compound was affective at preventing the collapse of the clay structure upon heating in the BK3-166B organoclay but no further characterization was performed to determine if there was an advantage in using this type of surface modification. It was assumed that the surfacatant used in these organoclays were able to withstand the cure temperature of the polyimides. 


\section{Conclusion}

Several combinations of monomers and organoclays were examined in an attempt to prepare well-dispersed polyimide/organoclay nanocomposites using an in-situ polymerization technique whereby poly(amide acid)s were synthesized in the presence of the organoclay. The organoclays that were used in this study were all considered low temperature ions and were given this distinction due to a common feature on all the surfactants; i.e. all had an aliphatic chain. The aliphatic chain was suspected to degrade at the temperatures necessary to cure the polyimides. Although this was the case, the use of these organoclays provided useful insight into the best mixing technique as well as the monomer/clay interaction in in-situ polymerization.

The various combinations of organoclay and polyimide presented in this chapter gave results that range from poor to high levels of clay dispersion. The difference in clay dispersion appeared to be somehow related to monomer structure. Some of the poly(amide acid) mixtures as well as the films prepared with organoclays via in-situ polymerization exhibited poor visual clarity indicating low levels of dispersion. Other polyimide/organoclay combinations exhibited good mixing and film clarity. The better combinations were further characterized yielding some very interesting results.

When certain organoclays such as SCPX-2003 were used with APB-BPDA and ODA-BTDA, poly(amide acid)/organoclay nanocomposite mixtures and films with a significant amount of exfoliation were prepared and characterized. Certain other organoclays such as the clays received from Triton and the two organoclays that were prepared in the lab followed the same general trends as the SCPX-2003 organoclay when 
placed in polyimides. Upon thermal conversion in air of poly(amide acid)/organoclay films to the corresponding polyimides, decomposition of the organic cation occurred resulting in the collapse to some degree of the clay particles into larger agglomerates. In addition, the films darkened with the decomposition. Thermal treatment under nitrogen allowed the interlayer cation to withstand higher temperatures but did not completely stop the degradation. The resulting polyimide/organoclay nanocomposite films still maintained a large amount of exfoliated clay particles regardless of cure atmosphere. Polyimide/organoclay nanocomposite films exhibited increased tensile moduli, similar strengths and lower elongations to break as compared to control films that contained no clay. It is apparent that organoclays with improved thermal stability are required for the preparation of polyimide/organoclay nanocomposites via a thermal imidization process regardless of cure atmosphere. 


\section{Chapter III References}

1) T. Lan, P. D. Kaviratna, and T. J. Pinnavaia. Chem. Mater. 1994;6:573.

2) T. Lan and T. J. Pinnavaia. Chem. Mater. 1994;6:216;

3) M. S. Wang and T. J. Pinnavaia. Chem. Mater. 1994;6:648;

4) T. Lan, P. D. Kaviratna, and T. J. Pinnavaia. Chem. Mater.1995;7: 2144.

5) T. J. Pinnavaia, T. Lan, P. D. Kaviratna, and M. Wang, Clay-Polymer Nanocomposites: Polyether and Polyimide Systems, Mater. Res. Soc. Symp., Paper N2.8 (1994);

6) P. D. Kaviratna, T. Lan, and T. J. Pinnavaia. Polym. Prepr. (Am. Chem. Soc., Polym. Div.) 1994;35:788.

7) E. R. Kleinfeld and G. S. Ferguson. Science 1994;265:370;

8) E. R. Kleinfeld and G. S. Ferguson. Chem. Mater 1995;7:2327.

9) P. B. Messersmith and E. P Giannellis. Chem. Mater. 1994;6:1719.

10) S. H. Burnside and E. P. Giannellis. Chem. Mater. 1995;7:1597.

11) P. B. Messersmith and E. P Giannellis. J. Polym. Sci A. 1996;33:1047.

12) Y. Fukushima, S. Inagaki. J. Inclusion Phenom. 1987;5:473.

13) Y. Fukushima, A. Okada, M. Kawasumi, T. Kurauchi, and O. Kamigaito. Clay Miner. $1988 ; 23: 27$.

14) A. Usuki, M. Kawasumi, Y. Kojima, A. Okada, T. Kurauchi, and O. Kamigaito. J. Mater. Res. 1993;8:1174.

15) A. Usuki, Y. Kojima, M. Kawasumi, A. Okada, Y. Fukushima, T. Kurauchi, and O. Kamigaito. J. Mater. Res. 1993;8:1179. 
16) Y. Kojima, A. Usuki, M. Kawasumi, A. Okada, Y. Fukushima, T. Kurauchi, and O. Kamigaito. J. Mater. Res. 1993;8:1185.

17) K. Yano, A. Usuki, A. Okada, T. Kurauchi, and O. Kamigaito. J. Polym. Sci., Polym. Chem. Ed. 1993;31:2493.

18) K. Yano, A. Usuki, A. Okada, T. Kurauchi, and O. Kamigaito. Polym. Prepr. (Am. Chem. Soc., Polym. Div.) 1991;32:65.

19) K. Yano, A. Usuki, and A. Okada, J. Polym. Sci., Part A: Polym. Sci. 1997;35:2289.

20) H. Tyan, Y. Liu, and K. Wie. Polymer 1999;40:4877.

21) J. Huang, Z. Zhu, J. Yin, X. Qian, and Y. Sun. Polymer 2000;42:873.

22) W. Xie, Z. Gao,W. Pan, R. Vaia, D. Hunter, A. Singh. Polym. Matl. Sci. and Eng. Proc. 2000;82:284.

23) Y. Yang, Z. Zhu, J. Yin, X. Wang, and Z. Qi. Polymer 1999;40:4407.

24) T. Agag, T. Koga, T. Takeichi. Polymer 2001;3399. 


\section{Chapter IV}

\section{In-situ polymerization with high temperature organoclays}

\section{Introduction}

Polyimide/organoclay nanocomposites can be prepared in a variety of ways with a host of different polymer-clay-surfactant combinations. The method most commonly reported for preparing polyimide/organoclay hybrids requires that a prepared poly(amide acid) solution be mechanically mixed with an organically modified montmorillonite ${ }^{l-18}$. The clays are usually modified with a quarternary amine having one or more hydrocarbon chains of 12 or more carbons ${ }^{19}$. The simple mixing methods provide materials with varying levels of dispersion including complete phase separation, intercalation, and exfoliation. Elaborate mixing techniques are sometimes used in order to achieve higher

levels of dispersion ${ }^{20}$. They may require special equipment. In-situ polymerization is an especially effective preparation method for polyimide nanocomposites with organically modified clays $^{21,22}$. However, as pointed out in the last chapter, thermal degradation of the organic surfactant has hindered progress in this area. Higher temperature organoclays that disperse as well as the aliphatic organoclays are needed in order to prepare quality polyimide nanocomposites.

The design of high temperature organoclays has been reported in recent publications ${ }^{23-26}$. In most cases where the use temperatures exceed $300^{\circ} \mathrm{C}$, an aromatic surfactant is employed. Using aromatic ions as surfactants pose unique problems in the preparation of polyimide nanocomposites. It is generally accepted that organoclays 
treated with aliphatic surfactants have better dispersion or are more easily dispersed in the polymer matrix than aromatic organoclays in nanocomposite preparation ${ }^{27}$. Attempts to make the organoclays containing aromatic ions perform better include providing one or more reactive endgroups on the surfactant for polymer tethering ${ }^{23-25}$. Other research is being performed in this area with other monofunctional amines, but there is not a clear understanding of what the best overall high temperature organoclay looks like ${ }^{26}$. A better fundamental understanding is needed of how the aromatic surfactant behaves on the clay surface and how the organoclays prepared with aromatic ions behave in polymer systems.

The focus of this work is to create and study organoclays that will disperse in polyimides and will not degrade at the cure temperature of the polyimide. To enhance the understanding of how the organoclays behave in solvent and in the nanocomposite mixtures, the surfactant-clay interaction is studied. Others have reported studies in this area. For example, Theng and coworkers measured the angle that pyridinium salts make with the surface of clay particles using infrared dichromism ${ }^{28}$. The orientation of trimethylphenylammonium on montmorillonite was also studied ${ }^{29}$. It was found that the aromatic salts make an angle between the plane of the aromatic ring and the clay surface that is not 90 degrees, indicating that aromatic rings have an affinity for the silicate oxide clay surface. This affinity is thought to be detrimental for the preparation of quality organoclays but there is not much research published in this area. Other work has been performed with aromatic species such as methylene blue which has an affinity for clay surfaces $^{30}$. Although the use of these aromatic ions for nanocomposite preparation was not thoroughly studied in these particular cases, the high temperature capabilities as well 
as the structure similarities with the polyimide backbone make them attractive for use in this work.

To create a completely homogenous polymer/clay system it is important to focus on the interface between the clay and the polymer. Vaia and coworkers ${ }^{27}$ have studied this interface between the bulk polymer and the clay surface. This modeling study provides interesting clues regarding clay surface modification. The study showed that increasing the area on the clay surface that can interact directly with the polymer greatly increases the chances of exfoliation. This free area on the clay surface is area that is void of surfactant. This along with the fact that greater separation between aromatic ions would leave more space for $\pi-\pi$ interactions between the surfactant ion and the polymer, pointed toward reducing the charge on the clay surface as a viable means of increasing exfoliation. This chapter reports on organically modified, reduced-charge clays, modified with high temperature surfactants. The results of these experiments were compared with those from fully charged clays with the same ion surfactant. The charge reduction was accomplished by lithium migration techniques ${ }^{31,32}$. The work presented herein provides an overview of the use of high temperature organoclays and possible surface modifications that make them more useful. 


\section{Experimental}

\subsection{Starting materials}

The organically modified clay (SCPX-2003) and Cloisite-(Na+) montmorrillonite were the two clays that were used in this section while APB, ODA, BPDA, and BTDA were obtained in polymer grade purity and used for polymer preparation.

High purity, anhydrous NMP, and 1,4 dioxane were purchased from Aldrich and used as received.

The following chemicals were purchased from Aldrich and used as received; trimethyl phenyl ammonium chloride (TMPA), 4-aminobiphenyl, tetraphenyl phosphonium chloride (TPP), aniline, triethyl phenyl ammonium chloride (TEPA), methyl iodide, and butyl iodide.

[4-(3-aminophenoxy)-4'-phenylethynylbenzophenone (3-APEB) m.p. 137-139 ${ }^{\circ} \mathrm{C}$ ] was prepared by Dr. Robert Bryant at NASA Langley Research Center and was recrystallized from 50/50 acetone/water. 


\subsection{Description of abbreviations}

The table below describes the different abbreviations used throughout the text below.

\begin{tabular}{|l|l|}
\hline Abbreviation & Description \\
\hline FCM-ion & $\begin{array}{l}\text { Fully charged montmorillonite. The ion describes the counterion on the clay } \\
\text { surface. }\end{array}$ \\
\hline RCM-250-ion & $\begin{array}{l}\text { Reduced Charge Montmorilonite-heated to } 250^{\circ} \mathrm{C} \text { and held for } 24 \mathrm{hrs} \text {. The } \\
\text { ion describes the counterion on the clay surface. }\end{array}$ \\
\hline RCM-2-ion & $\begin{array}{l}\text { Reduced Charge Montmorilonite-heated to } 250^{\circ} \mathrm{C} \text { and held for } 2 \mathrm{hrs} . \text { The } \\
\text { ion describes the counterion on the clay surface. }\end{array}$ \\
\hline RCM-3-ion & $\begin{array}{l}\text { Reduced Charge Montmorilonite-heated to } 250^{\circ} \mathrm{C} \text { and held for } 3 \mathrm{hrs} . \text { The } \\
\text { ion describes the counterion on the clay surface. }\end{array}$ \\
\hline RCM-6-ion & $\begin{array}{l}\text { Reduced Charge Montmorilonite-heated to } 250^{\circ} \mathrm{C} \text { and held for } 6 \mathrm{hrs} . \text { The } \\
\text { ion describes the counterion on the clay surface. }\end{array}$ \\
\hline MCM-120-ion & $\begin{array}{l}\text { Medium Charge Montmorilonite-heated to } 120^{\circ} \mathrm{C} \text { and held for } 24 \mathrm{hrs} \text {. The } \\
\text { ion describes the counterion on the clay surface. }\end{array}$ \\
\hline MCM-130-ion & $\begin{array}{l}\text { Medium Charge Montmorilonite-heated to } 130^{\circ} \mathrm{C} \text { and held for } 24 \mathrm{hrs} \text {. The } \\
\text { ion describes the counterion on the clay surface. }\end{array}$ \\
\hline
\end{tabular}

Table 1. Description of abbreviations

\subsection{Preparation of ions for ion exchange reactions}

The following is a representative procedure for preparing trimethyl biphenyl ammonium (BP) iodide.

Into a $100 \mathrm{~mL}$ three necked flask equipped with a stirring rod was placed 4aminobiphenyl $(5.0275 \mathrm{~g})$ and deionized water $(20 \mathrm{~mL})$. After stirring for 5 minutes iodomethane (16.75 g) was added to the flask. The white pasty mixture was heated to reflux and stirred overnight. Upon cooling a white precipitate remained in the reaction flask. The solution was filtered under vacuum in a Büchner funnel yielding an off-white 
powder. The powder was recrystallized from absolute ethanol yielding white crystals. The dried crystals melted at $219^{\circ} \mathrm{C}$.

The following is a representative procedure for preparing tributlyl phenyl ammonium (TBPA) iodide salts.

Into a $50 \mathrm{~mL}$ round bottom flask equipped with a condenser and a stir bar was placed aniline ( $2 \mathrm{~g}, 0.02$ moles) and iodobutane $(11.8557 \mathrm{~g}, 3$ moles). The mixture was stirred until dissolution and subsequently slowly heated to reflux while stirring. Upon heating, a white precipitate formed. The precipitate was isolated by filtration and washed with chloroform. The melting point of the white salt was $270^{\circ} \mathrm{C}$.

The following is a representative procedure for the preparation of the hydrochloride salt of ODA.

Into a $500 \mathrm{~mL}$ beaker was placed a $0.01 \mathrm{~N}$ hydrochloride salt solution $(350 \mathrm{~mL})$. The solution was stirred with a magnetic stir bar while ODA $(0.6130 \mathrm{~g})$ was added as a powder. The solution was heated to $60^{\circ} \mathrm{C}$ and stirred for 3 hours. During this time a rust brown solution formed. This solution containing the hydrochloride salt of ODA was used to prepare organoclays.

The following is a representative procedure used to prepare the hydrochloride salt of APEB.

Into a $25 \mathrm{~mL}$ beaker was placed APEB (1.5048 $\mathrm{g})$ and 1,4-dioxane $(20 \mathrm{~mL})$. Upon dissolution the solution turned yellow and was viscous. The solution was stirred 
with a magnetic stir bar for 0.5 hour. $\mathrm{A} 0.01 \mathrm{~N} \mathrm{HCl}$ solution was added dropwise while stirring until the solution was saturated. During the addition a precipitate formed but redissolved with stirring. The solution was then slowly added to a $12 \mathrm{~N} \mathrm{HCl}$ solution with stirring to afford a yellow precipitate. The precipitate was isolated by filtration, air dried, and stored in a dessicator.

The following is a representative procedure for preparing the dihydrochloride salt of APB.

Into a $25 \mathrm{~mL}$ beaker was placed APB $(3 \mathrm{~g})$ and 1,4 dioxane $(10 \mathrm{~mL})$. The mixture was stirred until the organic powder was completely dissolved. A $0.01 \mathrm{~N} \mathrm{HCl}$ solution was added dropwise ( $\sim 300$ drops) to the APB solution while stirring. The drops caused precipitation. With stirring, the white precipitate redissolved. The acid was added until the $\mathrm{pH}$ dropped significantly. The entire solution was precipitated in $12 \mathrm{~N} \mathrm{HCl}$ while stirring. The resulting precipitate was filtered through a sintered glass funnel under vacuum. The water-soluble salt was dried in flowing air.

\subsection{Preparation of variable charge clays}

The following are three examples of procedures used to prepare reduced charge montmorrillonite clays.

A). RCM-250: Into a $500 \mathrm{~mL}$ beaker was placed Cloisite $\mathrm{Na}+(5.0063 \mathrm{~g})$ and $250 \mathrm{~mL}$ of a $1 \mathrm{~N}$ lithium chloride solution. The clay suspension was homogenized for 5 minutes to give a homogenous suspension. The clay suspension was then placed in plastic 
centrifuge tubes and centrifuged at $4000 \mathrm{rpm}$ for 10 minutes yielding a clay pellet. The clay was then transferred to a $500 \mathrm{~mL}$ beaker where $250 \mathrm{~mL}$ of a $1 \mathrm{~N}$ lithium chloride solution was added. The suspension was homogenized. The clay was again centrifuged to a pellet. The clay was then resuspended via sonication, transferred to a cotton thimble, and extracted in a Soxhlet apparatus with ethanol (95\%) for 24 hours. The washed clay was transferred from the Soxhlet thimble to a platinum crucible and heated at $70^{\circ} \mathrm{C}$ for 1 hr to remove some of the ethanol. The crucible was then placed in a flowing air oven and heated to $110^{\circ} \mathrm{C}$ over $1 \mathrm{hr}$ and held at $110^{\circ} \mathrm{C}$ for $1 \mathrm{hr}$. The clay was then heated to 250 ${ }^{\circ} \mathrm{C}$ over $1 \mathrm{hr}$ and held at $250^{\circ} \mathrm{C}$ for $9 \mathrm{hrs}$. The clay was removed and ground in a mortar and pestle. The mass of the remaining clay was $4.4125 \mathrm{~g}$. The crushed clay was then washed 2 times in centrifuge tubes with a $1 \mathrm{~N}$ magnesium chloride solution. During the washing, the clay was sonicated to insure the interior of the clay was being effectively washed by the solution. The clay suspension was centrifuged at $4000 \mathrm{rpm}$ affording a clay pellet. The clay was then resuspended using sonication and washed two times accompanied by sonication with a $0.01 \mathrm{~N}$ magnesium chloride solution. The suspension was again centrifuged to a pellet and placed in a Soxhlet thimble and extracted overnight with ethanol $(95 \%)$. The clay was removed and suspended in a sodium chloride solution two times. The clay was placed in a Soxhlet thimble and extracted overnight with a $50 / 50(v / v)$ water/ethanol solution. The clay was then placed in a crucible and dried at 70 ${ }^{\circ} \mathrm{C}$ under vacuum for $3 \mathrm{hrs}$. The clay was ground to a fine powder and stored for use.

B). RCM 250-2,3,6: Into a $500 \mathrm{~mL}$ beaker was placed Cloisite $\mathrm{Na}+(10 \mathrm{~g})$ and $500 \mathrm{~mL}$ of a $1 \mathrm{~N}$ lithium chloride solution. The clay suspension was homogenized for 5 minutes 
until a homogenous suspension was reached. The clay suspension was then placed in a

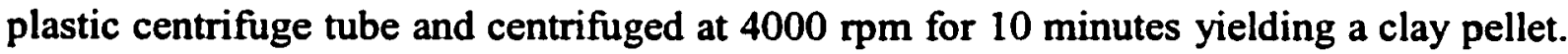
The clay was then transferred to a $500 \mathrm{~mL}$ beaker where $500 \mathrm{~mL}$ of a $1 \mathrm{~N}$ lithium chloride solution was added and again the suspension was homogenized. The clay was centrifuged to a pellet. The clay was then resuspended using sonication and washed with $95 \%$ ethanol three times. The washed clay was removed from the centrifuge tubes and equal amounts were placed in three separate platinum crucibles. The clay was heated at $70{ }^{\circ} \mathrm{C}$ for $1 \mathrm{hr}$ to remove some of the ethanol. The crucibles were subsequently placed in a flowing air oven and heated to $110^{\circ} \mathrm{C}$ over $\mathrm{hr}$ and held at $110^{\circ} \mathrm{C}$ for $1 \mathrm{hr}$. The clay was then heated to $250^{\circ} \mathrm{C}$ over $1 \mathrm{hr}$ and held at $250^{\circ} \mathrm{C}$. The clay was removed from the oven at different times. One of the samples was removed after $2 \mathrm{hrs,}$ one after $3 \mathrm{hrs}$ and one after 6 hrs. The samples were ground to a fine powder after removal from the oven. All of the crushed clay samples were then washed 2 times in a centrifuge tube with a $1 \mathrm{~N}$ magnesium chloride solution. While washing, the clay was sonicated to ensure the clay interior was being effectively washed by the solution. The clay suspension was centrifuged at $4000 \mathrm{rpm}$ creating a clay pellet. The clay was then resuspended and washed 2 times accompanied by sonication with a $0.01 \mathrm{~N}$ magnesium chloride solution. The suspension was again centrifuged to a pellet and placed in a soxhlet thimble and extracted overnight with ethanol (95\%). The clay was removed and suspended in a $1 \mathrm{~N}$ sodium chloride solution 2 times. The clay was placed in a soxhlet thimble and extracted overnight with a $50 / 50(\mathrm{v} / \mathrm{v})$ water/ethanol solution. The clay was placed in a crucible and dried at $70{ }^{\circ} \mathrm{C}$ under vacuum for $3 \mathrm{hrs}$. The clay was ground to a fine powder and stored for use. 
The samples were labeled RCM-2, RCM-3, and RCM-6 to indicate their hold times at $250^{\circ} \mathrm{C}$.

C). MCM-120 and MCM-130: Into a $500 \mathrm{~mL}$ beaker was placed Cloisite $\mathrm{Na}+(4 \mathrm{~g})$ and $250 \mathrm{~mL}$ of a $1 \mathrm{~N}$ lithium chloride solution. The clay suspension was homogenized for 5 minutes to give a homogenous suspension. The clay suspension was subsequently placed in plastic centrifuge tubes and centrifuged at $4000 \mathrm{rpm}$ for 10 minutes yielding clay pellets. The clay was then transferred to a $500 \mathrm{~mL}$ beaker where $250 \mathrm{~mL}$ of a $1 \mathrm{~N}$ lithium chloride solution was added and again the suspension was homogenized. After this washing in lithium chloride, the clay was centrifuged to a pellet. The clay was resuspended and washed with $95 \%$ ethanol three times. The clay was removed from the centrifuge tubes and equal amounts were placed in two separate platinum crucibles. The crucibles were then placed in a flowing air oven. One crucible was heated to $120^{\circ} \mathrm{C}$ and held there for $24 \mathrm{hrs}$ and the other was heated to $130^{\circ} \mathrm{C}$ and held there for $24 \mathrm{hrs}$. The clay samples were removed from the oven and both samples ground to a fine powder. The samples were washed two times with a $1 \mathrm{~N}$ sodium chloride solution. The samples were then washed with deionized water two times and then centrifuged to a pellet. The pellets were dried at $70^{\circ} \mathrm{C}$ under vacuum and bottled for use.

The samples were labeled MCM-120 and MCM-130 to indicate the temperature that they were held for 24 hrs. 


\subsection{Preparation of TMPA organoclays}

Into a $250 \mathrm{~mL}$ fleaker was placed Cloisite $\mathrm{Na}+(1.0026 \mathrm{~g})$ and $100 \mathrm{~mL}$ of deionized water. The clay was homogenized until a sol or stable suspension was reached. In a separate $25 \mathrm{~mL}$ beaker was placed trimethyl phenyl ammonium $(0.2385 \mathrm{~g})$ and $20 \mathrm{~mL}$ of deionized water. The salt was stirred in the water with a magnetic stir bar until the salt dissolved. The salt solution was added dropwise to the clay suspension while homogenizing. The mixture was homogenized after the salt was added for approximately five minutes to insure good mixing. The entire mixture was subsequently transferred to a $1000 \mathrm{~mL}$ round bottom flask and $100 \mathrm{~mL}$ of deionized water was added. The flask was equipped with a condenser and a stir bar was added to the organoclay suspension. The flask was heated to $80^{\circ} \mathrm{C}$ over a stirrer plate for $3 \mathrm{hrs}$. The organoclay was filtered through a Soxhlet thimble. The clay was extracted for 24 hrs with a 50/50 (v/v) water/ethanol solution. The clay was placed in a quartz crucible and heated to $70{ }^{\circ} \mathrm{C}$ under vacuum for $3 \mathrm{hrs}$. The clay was ground to a fine powder with a mortar and pestle, placed in a glass bottle, and stored in a dessicator.

\subsection{Preparation of TEPA organoclays}

Into a $250 \mathrm{~mL}$ fleaker was placed Cloisite $\mathrm{Na}+(1.0020 \mathrm{~g})$ and deionized water (100 $\mathrm{mL}$ ). The clay was homogenized until a sol was reached. In a separate $25 \mathrm{~mL}$ beaker was placed triethyl phenyl ammonium $(0.4644 \mathrm{~g})$ and $20 \mathrm{~mL}$ of deionized water. The salt was stirred in the water with a magnetic stir bar until the salt dissolved. The mixture was added dropwise to the clay suspension while homogenizing. The clay suspension was 
homogenized after the salt was added for approximately five minutes to insure good mixing. The entire mixture was transferred to a $1000 \mathrm{~mL}$ round bottom flask and $100 \mathrm{~mL}$ of deionized water was added. The flask was equipped with a condenser and a stir bar was added to the suspension. The suspension was then heated to $80^{\circ} \mathrm{C}$ over a stirrer plate for 12 hrs. The solution was washed with deionized water via centrifugation and suspension. The clay was then placed in a quartz crucible and heated to $85^{\circ} \mathrm{C}$ in a flowing air oven. The clay was ground to a fine powder with a mortar and pestle, placed in a glass bottle, and stored in a dessicator.

\subsection{Preparation of TBPA organoclays}

Into a $250 \mathrm{~mL}$ fleaker was placed Cloisite $\mathrm{Na}+(1.0026 \mathrm{~g})$ and $100 \mathrm{~mL}$ of deionized water. The clay was homogenized until a stable suspension was reached. In a separate $25 \mathrm{~mL}$ beaker was placed trimethyl phenyl ammonium $(0.2385 \mathrm{~g})$ and $20 \mathrm{~mL}$ of deionized water. The salt was stirred in the water with a magnetic stir bar until the salt dissolved. The salt solution was added dropwise to the clay suspension while homogenizing. The mixture was homogenized after the salt was added for approximately five minutes to insure good mixing. The entire mixture was subsequently transferred to a $1000 \mathrm{~mL}$ round bottom flask and $100 \mathrm{~mL}$ of deionized water was added. The flask was equipped with a condenser and a stir bar was added to the suspension. The suspension was heated to $80^{\circ} \mathrm{C}$ over a stirrer plate for $3 \mathrm{hrs}$. The organoclay suspension was filtered through a Soxhlet thimble. The clay was extracted for 24 hrs with a $50 / 50$ (v/v) water/ethanol solution. The clay was then placed in a quartz crucible and heated to $70^{\circ} \mathrm{C}$ 
under vacuum for $3 \mathrm{hrs}$. The clay was ground to a fine powder with a mortar and pestle, placed in a glass bottle, and stored in a dessicator.

\subsection{Preparation of BP organoclays}

Into a $250 \mathrm{~mL}$ fleaker was placed Cloisite $\mathrm{Na}+(1.00 \mathrm{~g})$ and $100 \mathrm{ml}$ of deionized water. The suspension was homogenized until a stable sol was reached. In a separate $250 \mathrm{~mL}$ beaker was placed BP cation $(0.4304 \mathrm{~g})$ and $200 \mathrm{ml}$ deionized water. The mixture was heated until the salt dissolved. The solution was subsequently added to the clay suspension and homogenized for five minutes. The entire mixture was then transferred to a $1000 \mathrm{~mL}$ round bottom flask and $100 \mathrm{~mL}$ of deionized water was added. The flask was equipped with a condenser and a stir bar was added to the suspension. The organoclay suspension was heated to $80^{\circ} \mathrm{C}$ over a stirrer plate for $12 \mathrm{hrs}$. The organoclay was washed with deionized water via centrifugation and suspension. The clay was then placed in a quartz crucible and heated to $85^{\circ} \mathrm{C}$ in a flowing air oven. The clay was ground to a fine powder with a mortar and pestle, placed in a glass bottle, and stored in a dessicator.

\subsection{Preparation of TPP organoclays}

Into a $250 \mathrm{~mL}$ fleaker was placed Cloisite $\mathrm{Na}+(0.5034 \mathrm{~g})$ and $100 \mathrm{~mL}$ of deionized water. The clay was homogenized until a stable suspension was reached. In a separate $25 \mathrm{~mL}$ beaker was placed TPP $(0.3771 \mathrm{~g})$ and $20 \mathrm{~mL}$ of deionized water. The salt was stirred in the water with a magnetic stir bar until the salt dissolved. The salt solution was 
added dropwise to the clay suspension while homogenizing. The mixture was homogenized after the salt was added for approximately five minutes to insure good mixing. The entire mixture was then transferred to a $1000 \mathrm{~mL}$ round bottom flask and $100 \mathrm{~mL}$ of deionized water was added. The flask was equipped with a condenser and a stir bar was added to the suspension. The suspension was then heated to $80{ }^{\circ} \mathrm{C}$ over a stirrer plate for 3 hrs. The organoclay suspension was then filtered through a Soxhlet thimble. The clay was extracted for $24 \mathrm{hrs}$ with a $50 / 50(\mathrm{v} / \mathrm{v})$ water/ethanol solution. The clay was then placed in a quartz crucible and heated to $70^{\circ} \mathrm{C}$ under vacuum for $3 \mathrm{hrs}$. The clay was ground to a fine powder with a mortar and pestle, placed in a glass bottle, and stored in a dessicator.

\subsection{Preparation of ODA organoclays}

Into a $250 \mathrm{~mL}$ fleaker was placed Cloisite $\mathrm{Na}+(1.08 \mathrm{~g})$ and $100 \mathrm{~mL}$ of deionized water. The clay was homogenized until a sol was reached. In a separate $250 \mathrm{~mL}$ beaker was placed $\mathrm{ODA}^{+}(\sim 0.4 \mathrm{~g})$ and $100 \mathrm{~mL}$ of a $0.1 \mathrm{~N} \mathrm{HCl}$ solution. The salt solution was added dropwise to the clay suspension while homogenizing. The clay suspension was homogenized after the salt was added for approximately five minutes to insure good mixing. The entire mixture was then transferred to a $1000 \mathrm{~mL}$ round bottom flask and $100 \mathrm{~mL}$ of deionized water was added. The flask was equipped with a condenser and a stir bar was added to the suspension. The organoclay suspension was then heated with a heating mantle to $80^{\circ} \mathrm{C}$ over a stirrer plate for $3 \mathrm{hrs}$. The suspension was then filtered through a Soxhlet thimble. The clay was extracted for 48 hrs with a $50 / 50(\mathrm{v} / \mathrm{v})$ water/ethanol solution. The clay was then placed in a quartz crucible and heated to $70{ }^{\circ} \mathrm{C}$ 
under vacuum for 3 hrs. The clay was ground to a fine powder with a mortar and pestle, placed in a glass bottle, and stored in a dessicator.

\subsection{Preparation of APEB organoclays}

Into a $250 \mathrm{~mL}$ fleaker was placed Cloisite $\mathrm{Na}^{+}(1.0137 \mathrm{~g})$ and $100 \mathrm{ml}$ of $95 \%$ ethanol. The mixture was homogenized until a stable suspension was reached. APEB salt $(\sim 0.5 \mathrm{~g})$ was placed in a $50 \mathrm{~mL}$ with $40 \mathrm{~mL}$ of hot $95 \%$ ethanol. The salt solution was added to the clay suspension and homogenized for $\sim 5$ minutes. The entire mixture was then transferred to a $1000 \mathrm{~mL}$ round bottom flask and $100 \mathrm{~mL}$ of EtOH was added. The flask was equipped with a condenser and a stir bar was added to the mixture. The organoclay suspension was heated to $80^{\circ} \mathrm{C}$ over a stirrer plate for $3 \mathrm{hrs}$. The suspension was filtered through a Soxhlet thimble. The clay was extracted for 48 hrs with $95 \%$ ethanol. The clay was then placed in a quartz crucible and heated to $70{ }^{\circ} \mathrm{C}$ under vacuum for $3 \mathrm{hrs}$. The clay was ground to a fine powder with a mortar and pestle, placed in a glass bottle, and stored in a dessicator.

\subsection{Preparation of APB organoclays}

Into a $250 \mathrm{~mL}$ fleaker was placed Cloisite $\mathrm{Na}+(1.0026 \mathrm{~g})$ and $100 \mathrm{~mL}$ of deionized water. The clay was homogenized until a stable suspension was reached $(\sim 5$ minutes). In a separate $25 \mathrm{~mL}$ beaker was placed the dihydrochloride salt of APB (0.6 $\mathrm{g})$ and $20 \mathrm{~mL}$ of deionized water. The salt completely dissolved with stirring using a magnetic stir bar. The resulting solution was then added dropwise to the clay suspension while homogenizing. The suspension was homogenized for $\sim 5$ minutes to insure good 
mixing. $100 \mathrm{ml}$ of deionized water was added to the clay suspension and the suspension was heated for $12 \mathrm{hrs}$ at $70^{\circ} \mathrm{C}$. This mixture was then introduced to a Soxhlet thimble and extracted for $24 \mathrm{hrs}$ with deionized water. The clay was dried under vacuum at 70 ${ }^{\circ} \mathrm{C}$.

2.13. Preparation of poly(amide acid)s

Refer to Section 2.6 in Chapter II.

2.14. Preparation of poly(amide acid)/organoclay hybrids via in-situ polymerization Refer to Section 2.5 in Chapter III.

2.15. Preparation of poly(amide acid)/organoclay hybrids via in-situ polymerization with organoclays that have been modified with diaminodihydrochloride salts

The following is a representative procedure used to prepare some poly(amide acid)/organoclay hybrid mixtures by synthesizing the poly(amide acid) in the presence of a clay that has two reactive amine groups.

Into a $100-\mathrm{ml}$ volumetric flask was placed FCM-APB $(0.1519 \mathrm{~g})$ and NMP $(10 \mathrm{~mL})$. The flask was fitted with a glass stopper and the flask was placed in a sonicator bath for $\sim 2 \mathrm{hrs}$ at room temperature to give a viscous yellow suspension. Into a 100 -ml threeneck round-bottom flask equipped with mechanical stirrer, nitrogen inlet, and drying tube containing calcium sulfate were placed the organoclay suspension, BPDA $(0.0393 \mathrm{~g}$, $0.134 \mathrm{mmol}$ ), and $5 \mathrm{~mL} \mathrm{NMP}$. The mixture was stirred until the dianhydride dissolved 
( $0.5 \mathrm{hrs})$. APB (2.4978 $\mathrm{g}, 8.544 \mathrm{mmol})$ and NMP ( $5 \mathrm{~mL})$ were subsequently added with mixing until the diamine dissolved $(\sim 0.5 \mathrm{hrs})$. Then more BPDA $(2.5139 \mathrm{~g}, 8.544 \mathrm{mmol})$ and NMP ( $5 \mathrm{~mL}$ ) were added to bring the concentration to $16 \%$ solids. The mixture was stirred at room temperature for $24 \mathrm{hrs}$ under nitrogen. The resulting yellow mixture was viscous and cloudy.

\subsection{Unoriented Thin Films}

Refer to Section 2.7 in Chapter II.

2.17. Unoriented thin films prepared from polyimide/organoclay mixtures where the organoclay and the polymer contain phenylethynyl end groups

Thin films were cast from neat (i.e. control) poly(amide acid) and poly(amide acid)/organoclay mixtures in NMP. The solution or mixture was doctored onto clean, dry plate-glass and dried at room temperature to a tack-free form in a low humidity chamber. The films were subsequently treated under several different drying conditions in flowing air and characterized. The thermal conditions after air drying were: (1) $100{ }^{\circ} \mathrm{C}$ for $1 \mathrm{hr}$; (2) $1 \mathrm{hr}$ each at $100^{\circ} \mathrm{C}$ and $200^{\circ} \mathrm{C}$; (3) $1 \mathrm{hr}$ each at 100,200 , and $300^{\circ} \mathrm{C}$ to effect solvent removal and imidization; (4) and $1 \mathrm{hr}$ each at $100,200,300$, and $371^{\circ} \mathrm{C}$ to crosslink the phenylethynyl terminated end groups. Film samples were removed from the plate glass and characterized. Thin-film tensile properties were determined according to ASTM D882 at room temperature using five specimens. 
2.18. Visual analysis of organoclay/polyimide thin films

A rating system was developed that put films in a systematic order according to their visual appearance. The person doing the research performed the analyses, thus the results are very subjective. Although this was not a perfect process, it allowed a more accurate assessment of the data from other characterization techniques on the same film.

The rating system described below gives some criteria for distinguishing levels of dispersion in the nanocomposite films. All the films being rated have approximately the same concentration of clay and that their thickness is approximately the same.

\begin{tabular}{|c|l|}
\hline Rating & \multicolumn{1}{|c|}{ Description } \\
\hline 1 & $\begin{array}{l}\text { An equal or better appearance than the neat polymer with a } \\
\text { glossy finish and no added color. }\end{array}$ \\
\hline 2 & $\begin{array}{l}\text { A siight color change from the neat polymer but no alteration } \\
\text { in the consistency of the film. }\end{array}$ \\
\hline 3 & $\begin{array}{l}\text { A hazy appearance but containing no visually separable clay } \\
\text { agglomerates. }\end{array}$ \\
\hline 4 & $\begin{array}{l}\text { Many visually separable clay agglomerates that are evenly } \\
\text { dispersed throughout the film. }\end{array}$ \\
\hline 5 & $\begin{array}{l}\text { Large agglomerates of clay that are not evenly dispersed } \\
\text { leaving large areas of the polymer film where there is no clay } \\
\text { at all. }\end{array}$ \\
\hline
\end{tabular}

Table 2. Visual analysis rating system

2.19. TEM analysis of nanocomposite thin films

TEM is a very useful tool for examining the dispersion characteristics of nanocomposite thin films. The TEM micrographs show the clay particles and their orientation in the final hybrid film. The micrographs are representative of only a very small area of the film and care must be taken to represent the overall dispersion 
characteristics of the nanocomposite film. The amount of clay present in a certain micrograph taken at a certain magnification is indicative of the level of dispersion of the organoclay. For example when comparing two micrographs taken at $3400 x$ magnification the micrograph with a larger amount of discrete particles represents the film where the clay is better dispersed. The TEM micrographs presented for the nanocomposite systems below are all taken at two different magnifications for each sample. The pictures on the left for all samples are taken at $22000 x$ magnification and the ones on the right for each sample are at $3400 x$ magnification. The pictures were taken of regions of the film that represented the entire film.

\subsection{Other Characterization}

Refer to Section 2.7 in Chapter III.

\section{Results and Discussion}

\subsection{Preparation of organic cations for clay surface modification}

Tributyl phenyl ammonium iodide (TBPA) and trimethyl biphenyl (BP) iodide were prepared

by reacting aniline with iodobutane and 4-aminobiphenyl with iodomethane, respectively (Section 2.3). There was some difficulty encountered in the preparation of these ions including the removal of the excess iodobutane in the preparation of TBPA. Using chloroform solved this problem. The first attempts at preparing the BP ion involved the 
use of a base which later caused problems in separating the base from the product. Doing the reaction without using base removed the separation step.

The hydrochloride salts of 4,4"-oxydianiline (ODA), 4-(3-aminophenoxy)-4'phenylethynylbenzophenone (3-APEB), and 1,3-bis(3-aminophenoxy)benzene (APB) were synthesized from the amine and hydrochloride acid (Section 2.3). The ODA dissolved in aqueous acid while the APEB and APB was first dissolved in water-miscible 1,4-dioxane.

\subsection{Preparation of variable charge clays}

Organoclays for use in polymers should have their platelets separated by organic cations creating a d-spacings that is favorable for polymer entrance ${ }^{27}$. This is normally accomplished in non- aromatic polymer systems by using clay surfaces that have a high charge density. Clays with high charge density combined with a surfactant having long aliphatic chains form a system where energy minimum occurs when the chains align themselves perpendicular to the clay surface. In some cases the high charge density facilitates a double layer of cations between adjacent clay sheets ${ }^{33}$. Although this is desirable for separating clay layers it is not desirable in creating free area on the clay surface for polymer clay interaction. That is, there is a tradeoff between clay-polymer interactions and layer separation when designing suitable organoclays for a polymer system. To study this balance, a fully charged clay with a cation exchange capacity (CEC) of $0.90 \mathrm{meq} / \mathrm{g}$ was altered to lower CEC values. The reduced charge clays, with their lower surface density of surfactant molecules, were then used in organoclay 
preparation and were compared with the fully charged organoclays for dispersion behavior.

The charge on montmorillonite clay surfaces is produced by imperfections in the crystal lattice ${ }^{31}$. The lattice is composed of an octahedral layer of metal ions sandwiched between two tetrahedral layers of silicon dioxide. The metal layer is usually made of aluminum ions with a coordination number of eight. In naturally occurring clays, some of these aluminum atoms $(+3)$ are replaced by magnesium $(+2)$ or lithium $(+1)$. This creates a negative charge imbalance that is manifest at the clay surface. This negative surface charge is compensated for in natural clays by sodium ions that enter the interlayer region. In order to reduce the charge on clays researchers have replaced these sodium ions with lithium ions and heated the clays at high temperatures ${ }^{31}$. The lithium ion enters the octahedral layer of the clay and becomes a permanent part of the clay. Importantly, the negative charge at the clay surface is reduced.

Reducing the charge on montmorillonite clays requires fewer interlayer cations and, thus, more of the clay surface is available for polymer interaction. The free space would allow for more $\pi$ - $\pi$ interactions between the aromatic organic cation and the aromatic rings of monomer and polymer molecules. To accomplish this charge reduction, lithium ions were incorporated in Cloisite- $\mathrm{Na}+$ via ion-exchange. The clay was subsequently heated to $250^{\circ} \mathrm{C}$ and held for $9 \mathrm{hrs}$ after washing and drying. It was important to perform this heating in crucibles that contain no sodium ions, thus platinum crucibles were used. This heat treatment yielded gray clay that was extremely hard and difficult to grind. It has been shown that this heat treatment collapses some of the clay layers and renders them virtually non-expandable in ion-exchange reactions with organic ions ${ }^{31}$. 
Instead, the more collapsed layers were expanded by introducing smaller inorganic magnesium ions by washing with different concentrations of magnesium chloride. After one washing with $1 \mathrm{~N}$ magnesium chloride and then $0.01 \mathrm{~N}$ magnesium chloride the interlayers that were made unexchangable during heat treatment were expanded and the ions in these layers were exchangeable. The magnesium washings were followed by sodium chloride washing to return the clay to the sodium form. These reduced charge clays were subsequently used in the preparation of organoclays.

Some of the films prepared with reduced charge organoclays showed only slightly better dispersion than films prepared with similar fully charged organoclays. While the fully charged clay was too highly charged, the reduced charge clay may have too low of a charge, so clays were prepared with intermediate charges. Initially hold time at $250^{\circ} \mathrm{C}$ was varied in the treatment with lithium. Three separate platinum crucibles were filled with fully charged clay that had been treated with lithium ions. These clay samples were placed in a flowing air oven and dried for one hour at $110^{\circ} \mathrm{C}$. The oven was then ramped to $250^{\circ} \mathrm{C}$ over one hour and held at this temperature. One of the clay containing crucibles was removed after two, three, and six hours. These reduced charge clays were again washed with the magnesium washings as described above and exchanged with sodium prior to use in organoclay preparation. The effect of the charge reduction was determined by each organoclays' performance in the nanocomposite. Different organoclays were prepared from these clays using various organic cations and then used in nanocomposite preparation with various polyimides. The nanocomposite preparations were characterized for the dispersion characteristics of the clay in polyimide materials. 
However, varying the hold time at $250{ }^{\circ} \mathrm{C}$ proved to be ineffective for producing clays with intermediate charge. Instead temperature was varied. Precedent for this approach can be found in the work of Giannellis and coworkers. They determined that a hold at 120 and $130{ }^{\circ} \mathrm{C}$ for 24 hours yields clays with CECs of 0.7 and $0.5 \mathrm{meq} / \mathrm{g}$ respectively ${ }^{30}$. The fully charged clay in their experiments had a CEC of $1.29 \mathrm{meq} / \mathrm{g}$. We prepared clays in like manner from Cloisite-Na+ with a CEC of $0.9 \mathrm{meq} / \mathrm{g}$. The lithium treatment was performed just as described for the RCM-250 clays, except that the clays were heated in platinum crucibles for $24 \mathrm{hrs}$ to 120 and $130{ }^{\circ} \mathrm{C}$. The clays produced in this manner did not become gray after heating but turned darker brown. These clays were not washed with magnesium prior to ion exchange with an organic ion because these clays were not expected to have collapsed when heated at these lower temperatures. The clay was exchanged with sodium and washed with water and centrifuged prior to conversion to the organoclay. Clays that were prepared in this manner were used in the preparation of organoclays with different organic cations, which, in turn, were used to prepare nanocomposites. Both the organoclay and the films were characterized.

\subsection{Preparation of organoclays}

Organoclays were prepared via ion-exchange reactions. The clays modified with inorganic ions such as sodium were changed to organically modified clays by exchanging the sodium with some organic ion. The clay that was used for all of the experiments discussed below was Cloisite $\mathrm{Na}+$ and the variably charged clays that were prepared from this clay. The Cloisite clay is a montmorrillonite with an exchange capacity of 0.90 
$\mathrm{meq} / \mathrm{g}$. This clay is very attractive for use in nanocomposite preparation because it is readily available as a commercial product. This clay is also thoroughly cleaned and the particle sizes are monitored rendering it consistent from one batch to the next. This clay has also been fully characterized so the sodium form of the clay is easily compared with the ion-exchanged form. The ion exchange procedures for the various organoclays are recorded in the experimental section.

In all the ion exchange reactions, a $1 \%$ clay suspension was prepared and mixed thoroughly until a stable suspension or sol was reached such that the clay particles remain suspended without stirring for approximately 30 minutes. Once this was accomplished a $5 \%$ solution of the organic salt was added dropwise to the suspension while homogenizing. The homogenizer was very effective at providing enough shearing force to separate the clay particles and promote the ion-exchange. The reaction is most commonly performed in water for those salts that are soluble in water. Water-insoluble salts were exchanged using suspensions of the clay and the salt in methanol or ethanol. In the exchange reactions with methanol (ODA+) or ethanol (APEB + ) the clay behaved differently than in water. In the sodium form the clay is very hydrophilic and remains in a stable sol in water. However, stable suspensions were not realized when $1 \%$ suspensions of the clay were prepared in either ethanol or methanol. Rather, in the absence of agitation the clay particles settle out rather quickly. The organoclays prepared from ion exchange reactions in methanol or ethanol also behaved differently. The clay did not flocculate into a fluffy powder but instead flocculated into little beads that sank to the bottom. The results from using alcohols as the ion exchange solvent were not as good as water but the alcohols were necessary when the organic ion was not soluble in water. 
Other modifications in organoclay preparation such as alternate mixing, washing, and drying techniques were examined for improvements in the existing preparatory techniques for organoclays. Mixing of the clays prior to and during the addition of the salt was usually done with a homogenizer. Sonication in a water bath was tried as an alternative to homogenization. This technique proved to be extremely effective at dispersing the sodium clay prior to addition of the salt solution. Stable suspensions or sols are easily achieved after sonicating clay/water mixtures. Alternate washing techniques were examined as well.

After ion exchange it is very important to remove any excess inorganic and organic salt from the clays prior to use. The most common way is to filter the sample and the wash the filter cake with large amounts of water until the sample is free of excess salt. This was not efficient and was not very practical on a small scale. This washing technique was replaced by Soxhlet extraction. The clay was placed in a double walled cellulose thimble and extracted overnight with water or a water/ethanol mixture. This provided an effective means of removing excess salt from the organoclay. To reduce the time of sample preparation this extraction procedure was sometimes replaced by repeated suspension and centrifugation of the samples in water. It is not clear whether this is as effective as Soxhlet extraction but was used in some cases. Subsequently, the clay is dried before use in nanocomposite preparation. Although heat treatment at temperatures around $70^{\circ} \mathrm{C}$ under vacuum was proven to be an effective means of drying organoclays, it was thought that freeze drying may be a better way to dry the organoclays. The use of heat to dry the organoclays may cause some reduction of the size of the clay galleries. These galleries need to be expanded by sonicating or homogenizing the organoclay in 
organic solvent for long periods of time when heat is used. This can be avoided by subliming the water off the clays instead of heating. Organoclays that are dried using heat need to be sonicated for hours in some cases to get the clay in the expanded state while clays that have been freeze dried disperse quickly in the sonicator in organic solvent. Some of the clays were prepared and subsequently dried using a freeze drying technique. Different combinations of these procedures were used to prepare organoclays for nanocomposite preparation.

The ions that were used in the preparation of organoclays are shown below in figure 1 .<smiles>CN(C)c1ccccc1</smiles>

TMPA

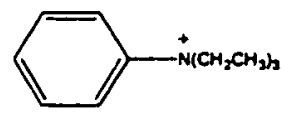

TEPA

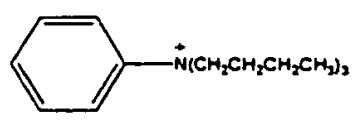

TBPA<smiles>Cc1ccc(Oc2ccc(C)cc2)cc1</smiles>

ODA+

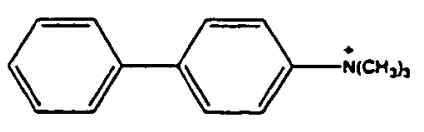

BP<smiles>c1ccc(C(c2ccccc2)(c2ccccc2)c2ccccc2)cc1</smiles>

TPP<smiles>Cc1cccc(Oc2cccc(Oc3cccc(C)c3)c2)c1</smiles>

APB<smiles>Nc1cccc(Oc2cccc(C(=O)c3cccc(C#Cc4ccccc4)c3)c2)c1</smiles>

APEB

Figure 1. Structure of cations used in organoclay preparation 


\subsection{Preparation of nanocomposites via in-situ polymerization}

An improved method for preparing polyimide/clay nanocomposites involved performing the polymerization of poly(amide acid)s in the presence of the clay as proposed by Yang et $\mathrm{al}^{22}$ and discussed in Chapter II. With this approach, at least a portion of the polymer should reside in clay gallery. The growth of the polymer, with its origin being in the gallery, could provide additional aid in forcing the clay layers apart resulting in exfoliation. The chemical structures of the monomers used in this study are depicted in Figure 2.<smiles>O=C1OC(=O)c2cc(-c3ccc4c(c3)C(=O)OC4=O)ccc21</smiles><smiles>Nc1cccc(Oc2cccc(Oc3cccc(N)c3)c2)c1</smiles><smiles>Nc1ccc(Oc2ccc(N)cc2)cc1</smiles><smiles>O=C(c1ccc2c(c1)C(=O)OC2=O)c1ccc2c(c1)C(=O)OC2=O</smiles>

Figure 2. Monomer structures

The monomers used in this study were those used to prepare the nanocomposites with the low temperature clays. This allowed for comparison between the two types of clays while the polymer remained constant. The results from characterization are described below. 
3.5. Preparation of poly(amide acid)/organoclay hybrids via in-situ polymerization with organoclays that have been modified with diaminodihydrochloride type salts

Organoclays were prepared from the dihydrochloride salts of the diamines and used in the preparation of hybrid mixtures. This type of surfactant not only functionalized the surface of the clay but also offered a site for attachment of the polymer chains themselves. This opens the possibility of an interlocking effect between the polymer and the surfactant, as some chains grow from the end of the surfactant while others fill gaps between the surfactant molecules. The charge on the organoclays was altered to test the effects of changing the amount of this type of surfactant. A change was made in the insitu polymerization of the PAA in certain experiments. The order of addition of the monomers was changed in one instance. The two different experimental designs are discussed below.

The first attempt at preparing these materials was to use a 1:1 stoichiometry as if the surfactant would not link to the polymer during polymerization. The clay was first combined with the APB and followed by the BPDA. A second attempt to exfoliate the layers in an APB-BPDA system with this type of organoclay was to dissolve the dianhydride in the presence of the clay prior to the diamine introduction. A 1:1 stoichiometry was used in this example as well. In this attempt to prepare well-dispersed materials, BPDA was added to the clay/solvent suspension prior to the addition of any diamine. It was thought this would react with the free amine on the surfactant and start expanding the clay layers prior to the addition of the rest of the diamine. This procedure did not cause any major changes in visual appearance of the PAA mixture or the 
nanocomposite film. Both of these techniques appeared to produce the same results for dispersion but more research is required to see which method works the best.

Characterization was performed on the polyimide films prepared from these mixtures.

3.6. XRD analysis of organoclays

As stated earlier it was important for this high temperature research to determine the orientation of the aromatic cations relative to the clay surface. To make this determination a series of aromatic cations were placed into cloisite $\mathrm{Na}+$ and the XRD patterns obtained. These XRD patterns for the various organoclays are shown in Figure 3.

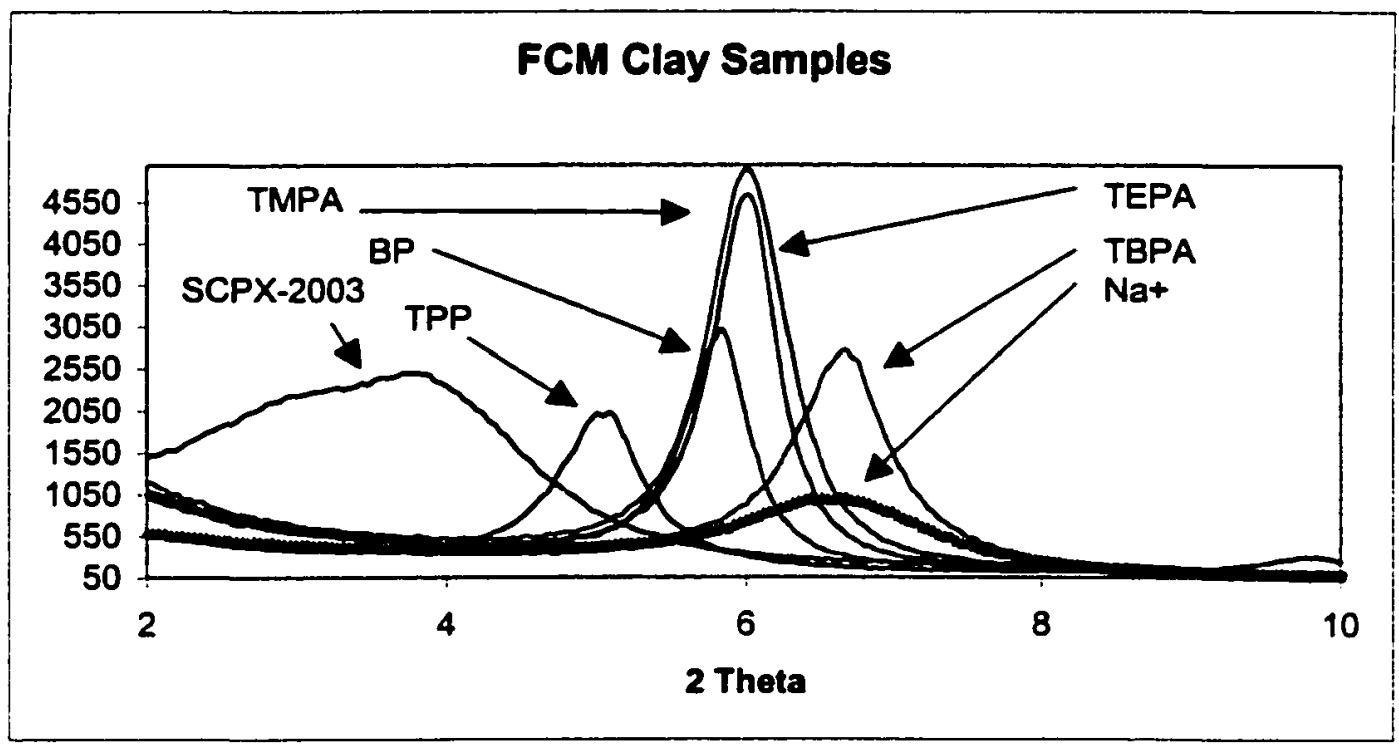

Figure 3. XRD patterns of fully charged organoclays

The SCPX-2003, an aliphatic organoclay, is included in this figure to compare the aromatic clays with the aliphatic organoclays. There is a large decrease in d-spacing in the organoclays that are exchanged with aromatic ions relative to the SCPX-2003. The 
peaks for the aromatic clays are close to one another except for the TBPA, which have dspacings similar to that of the sodium clay, and TPP organoclays, which have d-spacings closer to that of the SCPX-2003. The results of XRD studies on the various clays are summarized in Table 3. Also included in this table are the d-spacings found for films made with these clays. Details of the film studies are presented in the sections that follow.

\begin{tabular}{|c|c|c|c|c|c|c|}
\hline & \multicolumn{3}{|c|}{$\begin{array}{c}\text { Fully Charged Clays (FCM) } \\
\text { d-spacing (nm) }\end{array}$} & \multicolumn{3}{|c|}{$\begin{array}{c}\text { Reduced Charge Clays (RCM-250) } \\
\text { d-spacing (nm) }\end{array}$} \\
\hline & Clay Alone & APB-BPDA & ODA-BTDA & Clay Alone & APB-BPDA & ODA-BTDA \\
\hline $\begin{array}{l}\text { SCPX- } \\
2003\end{array}$ & 2.37 & 1.34 & 1.28 & & & \\
\hline $\mathrm{Na}$ & 1.33 & & & & & \\
\hline TMPA & 1.47 & 1.26 & 1.30 & 1.36 & 1.23 & 1.22 \\
\hline TEPA & 1.47 & & & & & \\
\hline TBPA & 1.32 & 1.17 & & & & \\
\hline ODA & No XRD & No XRD & & & & \\
\hline$\overline{\mathrm{APB}}$ & No XRD & 1.26 & & & No Peak & \\
\hline APEB & No XRD & No XRD & & & & \\
\hline TPP & 1.74 & 2.19 & 1.79 & $2.19,0.95$ & $1.94,0.95$ & 1.28 \\
\hline BP & 1.51 & 1.32 & 1.28 & 1.52 & 1.24 & 1.23 \\
\hline \multirow[t]{3}{*}{ DTMA } & 1.86 & 1.34 & & 1.39 & & \\
\hline & \multicolumn{3}{|c|}{ MCM-120-ion } & \multicolumn{3}{|c|}{ MCM-130-ion } \\
\hline & Clay Alone & APB-BPDA & ODA-BTDA & Clay Alone & APB-BPDA & ODA-BTDA \\
\hline TMPA & & & 1.28 & & & 1.28 \\
\hline
\end{tabular}

Table 3. Calculated d-spacings for organoclays alone and in hybrid films

The d-spacing for the TBPA organoclay cannot be explained in a way that would be consistent with the other organoclays discussed below. Among the other three aromatic organoclays there was no major difference in d-spacing. The clays that were exchanged with TMPA and TEPA were almost identical in layer spacing while the BP organoclay was slightly more expanaed. A diagram that would explain these layer spacings is shown in Figure 4. 

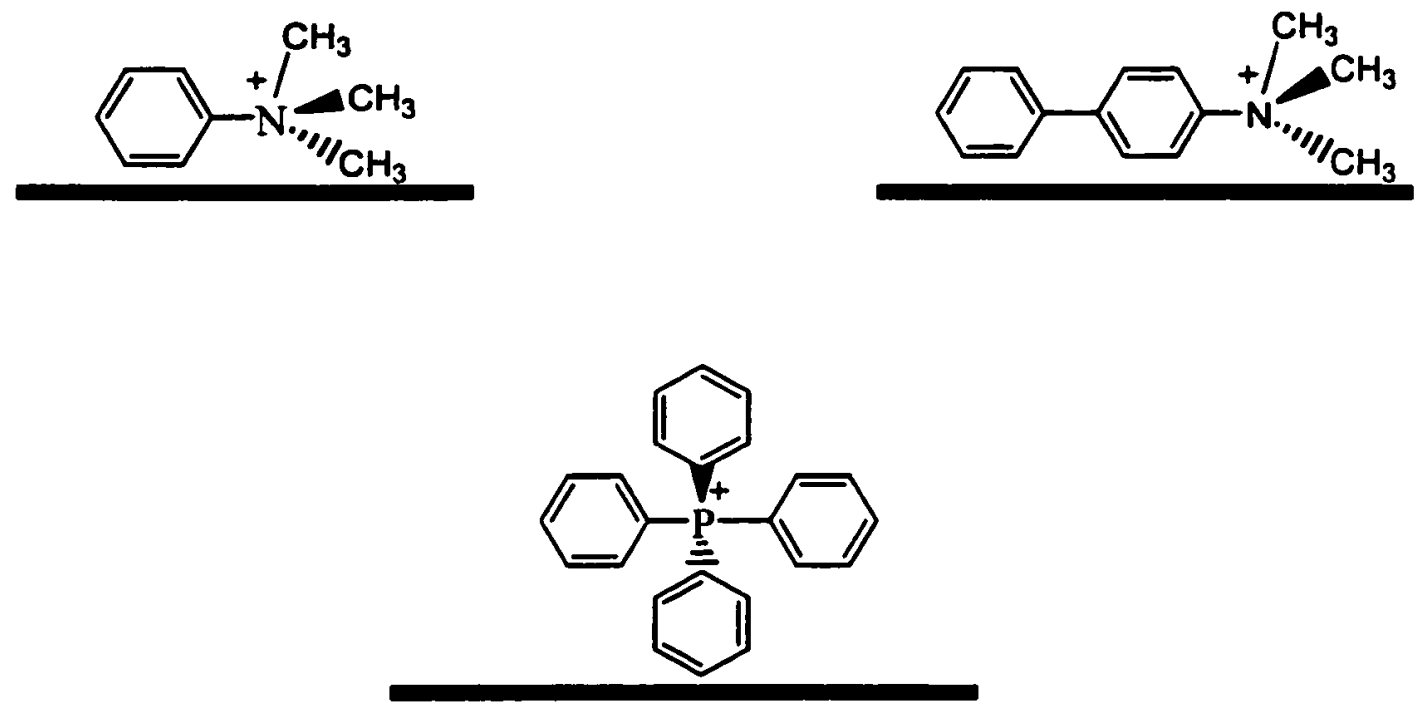

Figure 4. Diagram of cation orientation on clay surfaces

The d-spacing data shows that with the addition of a second phenyl ring (BP) there is not a large increase in d-spacing indicating that the aromatic portion of the ions are most likely as close to the surface as possible. If the aromatic rings were perpendicular to the surface then the addition of a second ring would create a larger d-spacing increase than $0.04 \mathrm{~nm}$. The tetrahedral arrangement of the phenyl rings on TPP does not allow for all of the rings to be against the surface. At least one of the rings has to be perpendicular to the surface in any arrangement of this cation on the surface. The d-spacing in this organoclay was larger as a result.

To examine the effect that clay charge reduction had on the d-spacing of the organoclays, XRD patterns were obtained on some of the reduced charge organoclays. Two organoclays were prepared from RCM-250-Na+ and the XRD patterns were obtained. The XRD patterns for RCM-250-TMPA and an organoclay that was prepared from RCM-250-Na+ exchanged with an ammonium salt containing an aliphatic tail of 12 
carbons (DTMA, see page 88 ) are shown in Figure 5. The XRD pattern for the RCM$250-\mathrm{Na}+$ clay is shown as well.

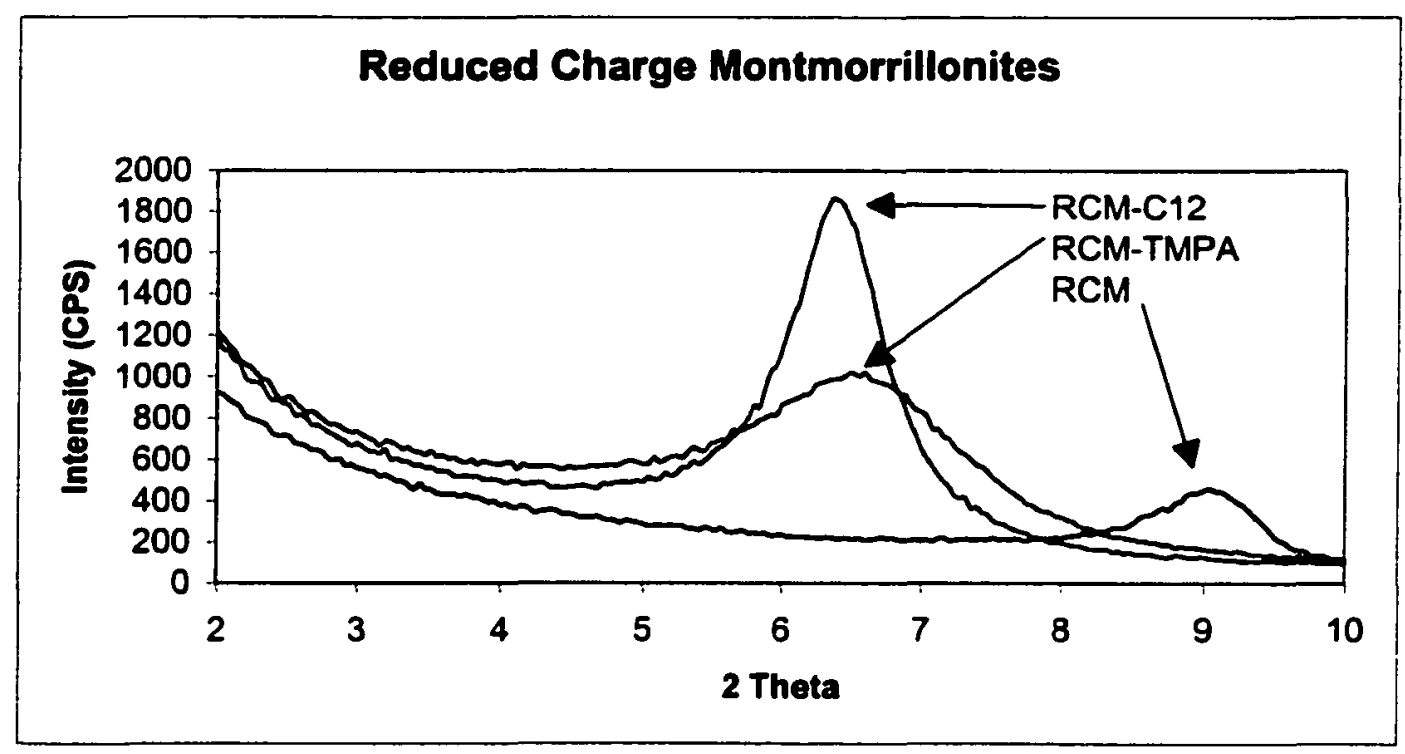

Figure 5. XRD pattern of reduced charge organoclays

The reduced charge sodium montmorillonite had a peak maximum at $2 \theta=9.04^{\circ}$ that corresponds to a d-spacing of $0.97 \mathrm{~nm}$. This very small d-spacing probably is the result of only a single layer of water molecules in the clay inner layer ${ }^{32}$. The pattern for the RCM-250-TMPA has a maximum at $2 \theta=6.48^{\circ}$ indicating a d-spacing of $1.36 \mathrm{~nm}$. This is smaller than the $\mathrm{d}$-spacing $(1.47 \mathrm{~nm})$ of the fully charged clay with this same ion. This could mean that there was less repulsive interaction between phenyl rings of oppositely faced clay sheets. The reduced repulsion allows the faces of the clay particles to come closer together and results in smaller layer spacings. This effect is demonstrated more clearly in the pattern where the dodecyl trimethyl ammonium salt is in the inner layer gallery. The charge on the clay's surface is so low that the aliphatic chains can actually lie flat on the clays surface. If this were a fully charged clay the chains would have to 
pack in the perpendicular position and the d-spacing would be larger. Figure 6 shows the XRD pattern of a fully charged organoclay prepared with the same C12 salt.

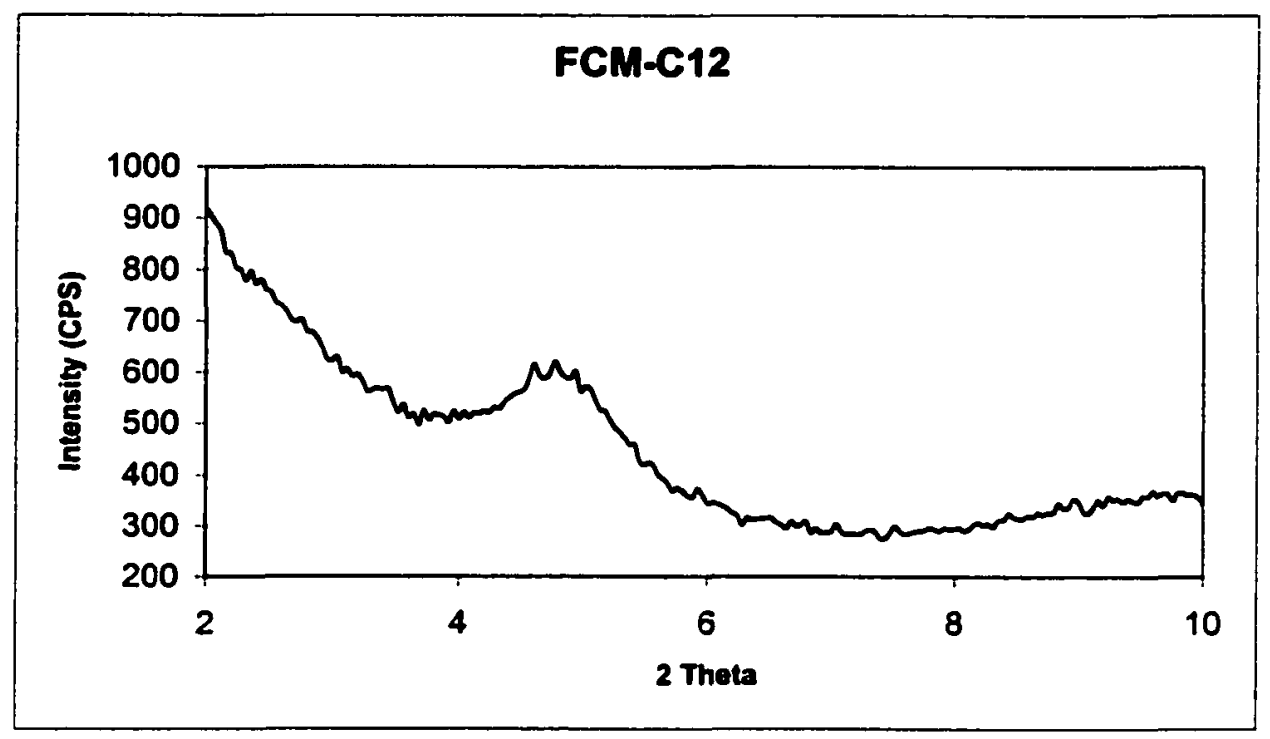

Figure 6. XRD pattern of fully charged organoclay prepared with an aliphatic amine The chains must be oriented perpendicular to the clay surface as shown by the peak position. The peak is at a lower angle indicating a larger $d$-spacing $(1.86 \mathrm{~nm})$. This clearly indicates that by reducing the charge on the clay's surface there is more area on the clay surface for organic interaction. This was seen as an advantage when preparing nanocomposite via in-situ polymerization because it allows for monomer interaction on the clay surface as well.

\subsection{XRD analysis of MCM-TMPA/ODA-BTDA hybrid films}

Polyimide/organoclay films were prepared with RCM-2-TMPA, RCM-3-TMPA and RCM-6-TMPA reduced-charge organoclays. The clays had been treated with lithium and held at $250^{\circ} \mathrm{C}$ for 2,3 , and 6 hours for these experiments (Section 2.4B). The clays 
were converted to the sodium form prior to exchange with TMPA. PAAvorganoclay mixtures were prepared via in-situ polymerization. The mixtures, which were cloudy after polymerization, were used to prepare polyimide films. The XRD pattern for these hybrid films prepared with the various organoclays dispersed in ODA-BTDA are shown below in Figure 7. Each film gave a single peak near $2 \theta=7.0^{\circ}$. Having the same dspacing for all of the films indicates that at least some particles had not dispersed. The data shown here does not indicate the dispersion level or the concentration of clay that was not exfoliated. It cannot be determined if the heating times had any effect on the dispersion properties of the clay from this data.

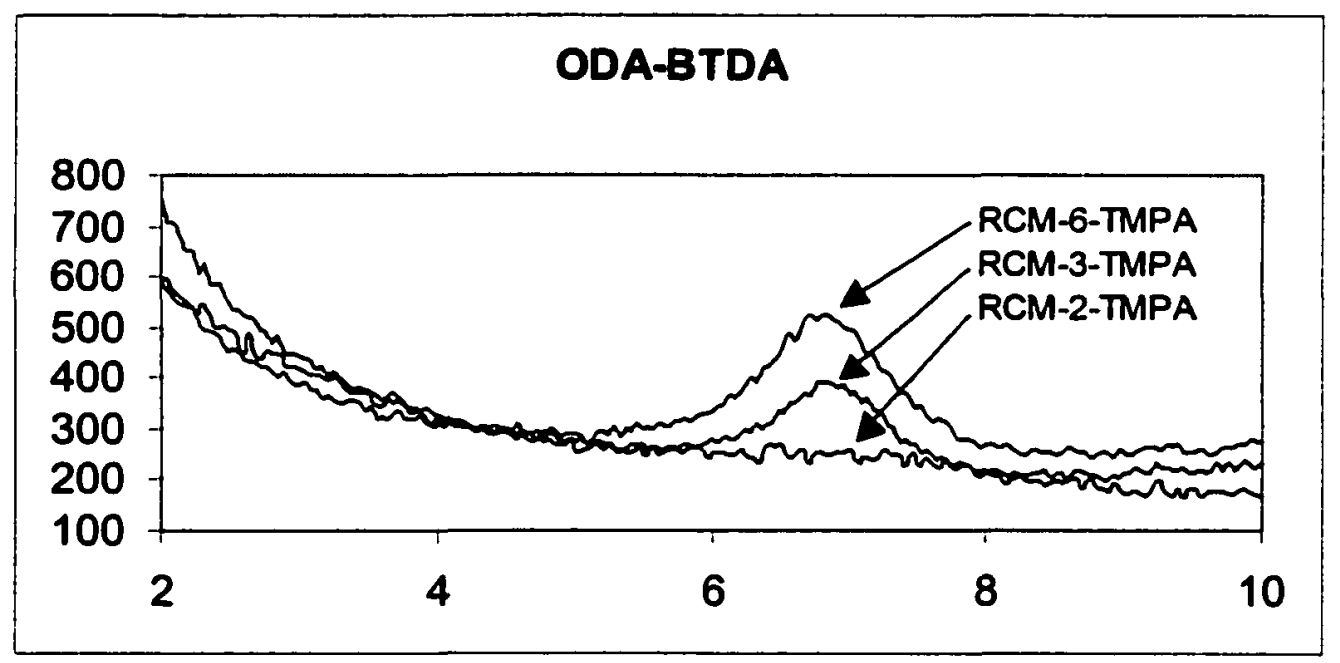

Figure 7. XRD pattern of RCM-2, 3, 6-TMPA ODA-BTDA hybrid films

Another attempt to prepare medium charged clays involved changing the heating temperature instead of the time. The clays were treated with lithium and heated to lower temperature than $250^{\circ} \mathrm{C}$ and held there for 24 hrs. The clay that was treated in this manner was then converted back to the sodium form. The sodium clay was exchanged with TMPA and used in nanocomposite preparation. The polymer that was used in these preliminary trials of this clay was again the ODA-BTDA polyimide. The first attempt at 
hybrid formation with these medium charge clays was with the MCM-120-TMPA organoclay. The hybrid mixture prepared with this organoclay was less cloudy than the other hybrid mixtures prepared with aromatic cations. The film prepared from this mixture also received a visual rating of 3 indicating better dispersion than the other films prepared with high temperature organoclays. The XRD of the 3\% MCM-120TMPA/ODA-BTDA film is shown below in Figure 8.

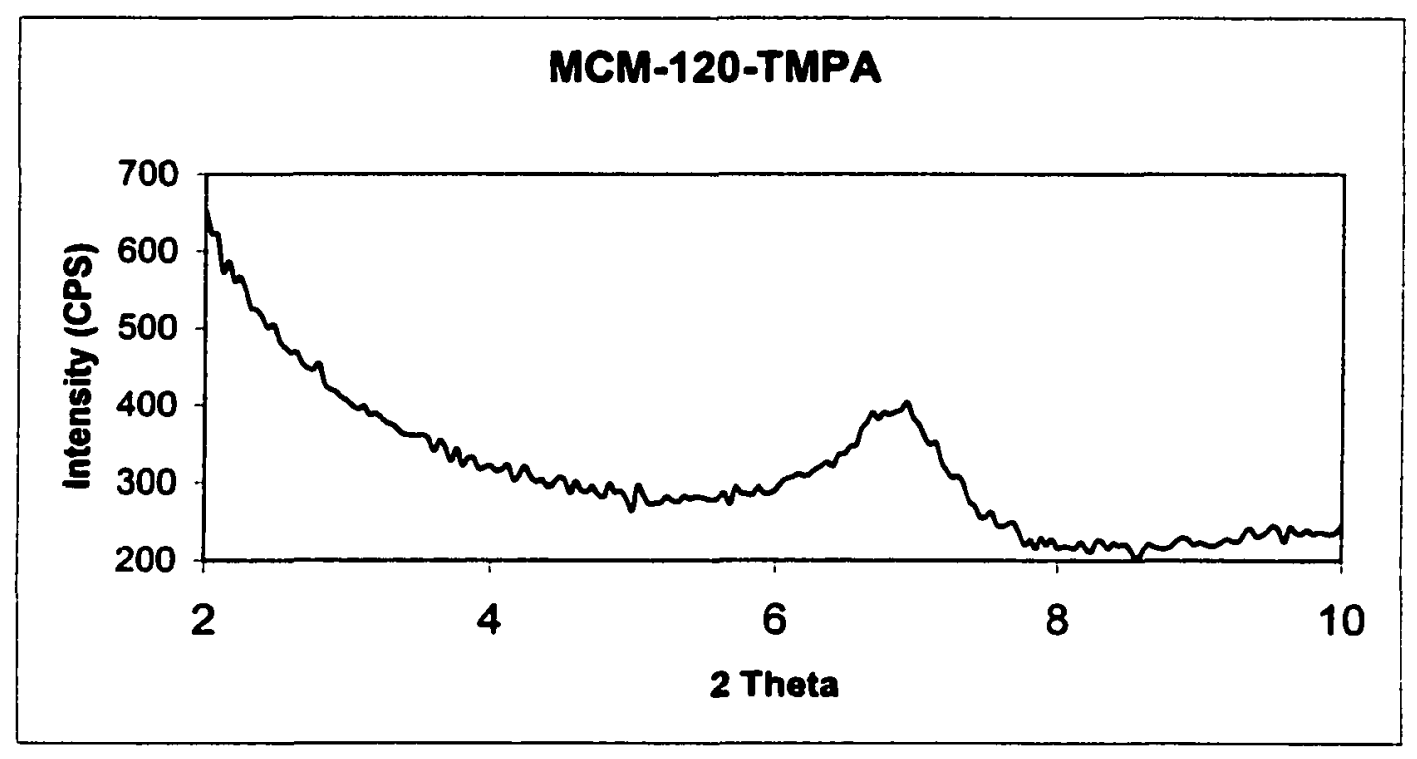

Figure 8. XRD pattern of MCM-120-TMPA/ODA-BTDA hybrid film

The XRD pattern of the hybrid film had a peak $2 \theta=6.92^{\circ}$ indicating a d-spacing of 1.28 $\mathrm{nm}$. The peak is not much different in position than the one for the other heat treatments but the films were much better in visual appearance, which may indicate that the clay was better dispersed and the peak was representative of a smaller amount of clay than in the other system. Another clay was prepared with the TMPA ion that had been treated with lithium and heated to $130^{\circ} \mathrm{C}$ for $24 \mathrm{hrs}$ (MCM-130-TMPA). This clay was also placed in ODA-BTDA using in-situ polymerization and after polymer formation the PAA mixture was similar in appearance to the MCM-120-TMPA. The film produced with this mixture 
was also given a visual rating of 3 . The film was examined with XRD and the pattern is presented in Figure 9.

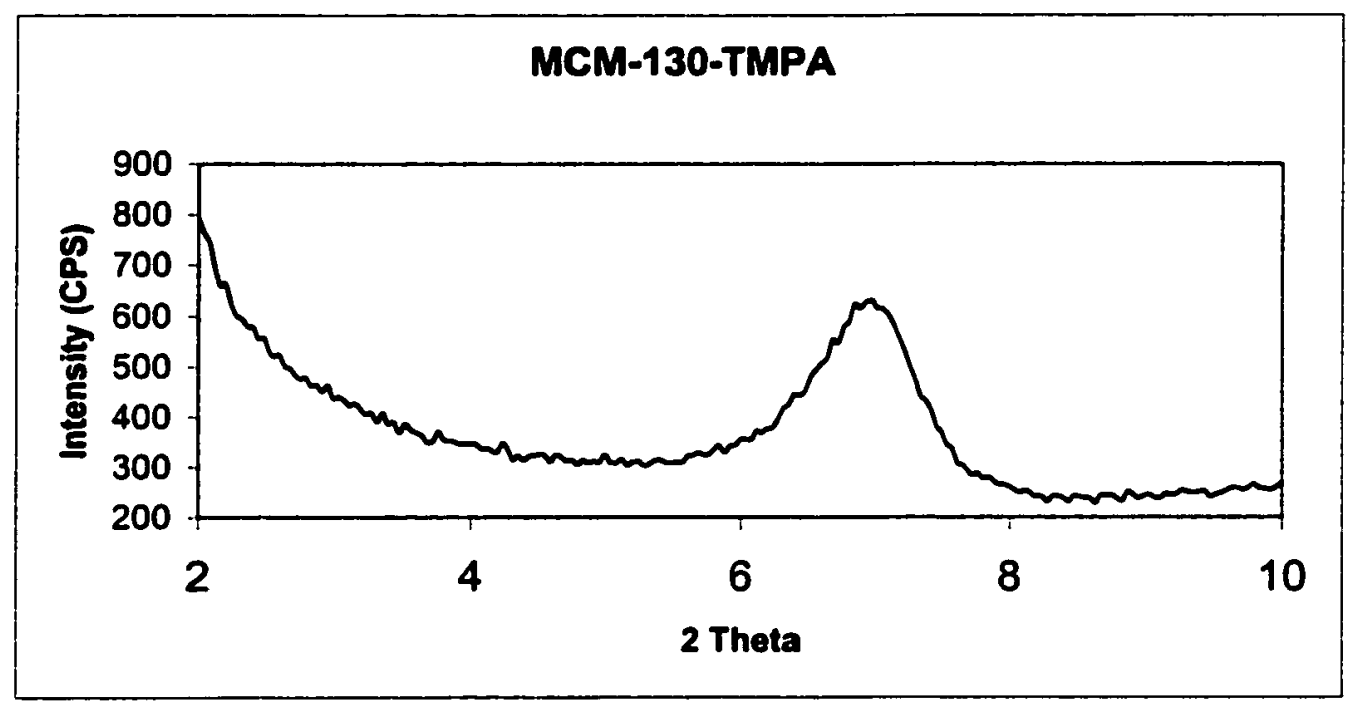

Figure 9. XRD pattern of MCM-130-TMPA/ODA-BTDA hybrid film

The hybrid film did exhibit a peak at the same place as the MCM-120-TMPA/ODABTDA hybrid. This indicated that there was some clay that was not separated during the polymerization. The medium charge clays did show some interesting results and further characterization needs to be performed in order to completely assess the usefulness of these clays.

\subsection{Characterization of FCM-ODA+/ODA-BTDA nanocomposites}

Hybrid materials were also prepared with FCM-ODA+ organoclays. The organoclay was placed in NMP and in-situ polymerization of ODA-BTDA polyimide was performed in the presence of the clay. The mixture was yellow and cloudy. A film was prepared from this hybrid mixture. The film showed that the clay did not disperse well in the 
polymer as clay agglomerates were readily seen by visual inspection. No further characterization of this hybrid was obtained.

\subsection{Characterization of FCM-APEB+/APB-BPDA and FCM-APEB+/APB-BPDA-} PEPA nanocomposites

A PAA mixture was prepared via in-situ polymerization with APEB+ organoclay and APB-BPDA polyimide. The mixtures were cloudy after polymer formation. Films were prepared from this mixture and heated to a final cure temperature of $300^{\circ} \mathrm{C}$. The films were not clear and clay particles were apparent to the unaided eye. No further characterization was performed. The APEB + was also used in the in-situ preparation of an APB-BPDA polyimide that was endcapped with PEPA. The PAA had a calculated molecular weight of $10,000 \mathrm{~g} / \mathrm{mol}$. The mixture was yellow and cloudy after stirring for $24 \mathrm{hrs}$. A film was poured from the hybrid mixture and after curing to $371^{\circ} \mathrm{C}$ the hybrid film also had many clay agglomerates that were visually apparent. No further characterization was performed with this film.

\subsection{Characterization of FCM-APB nanocomposites}

Nanocomposites were prepared with fully charged montmorillonite clays that were modified with the dihydrochloride salt of APB. The organoclays that were used were prepared from Cloisite $\mathrm{Na}+$ via ion exchange of the sodium with $\mathrm{APB}+$ in an aqueous suspension. The organoclays had a reddish tint after heating, washing, and drying. The organoclays were suspended in NMP and after sonicating for $\sim 0.5 \mathrm{hr}$ yielded a yellow, viscous suspension. APB-BPDA polyimide was prepared in the presence of the clay 
yielding a cloudy, yellow-red mixture. The viscous mixture was used to prepare thin films and the films were characterized. The film received a visual rating of 2 . The film was also characterized with XRD and the pattern is shown below in Figure 10.

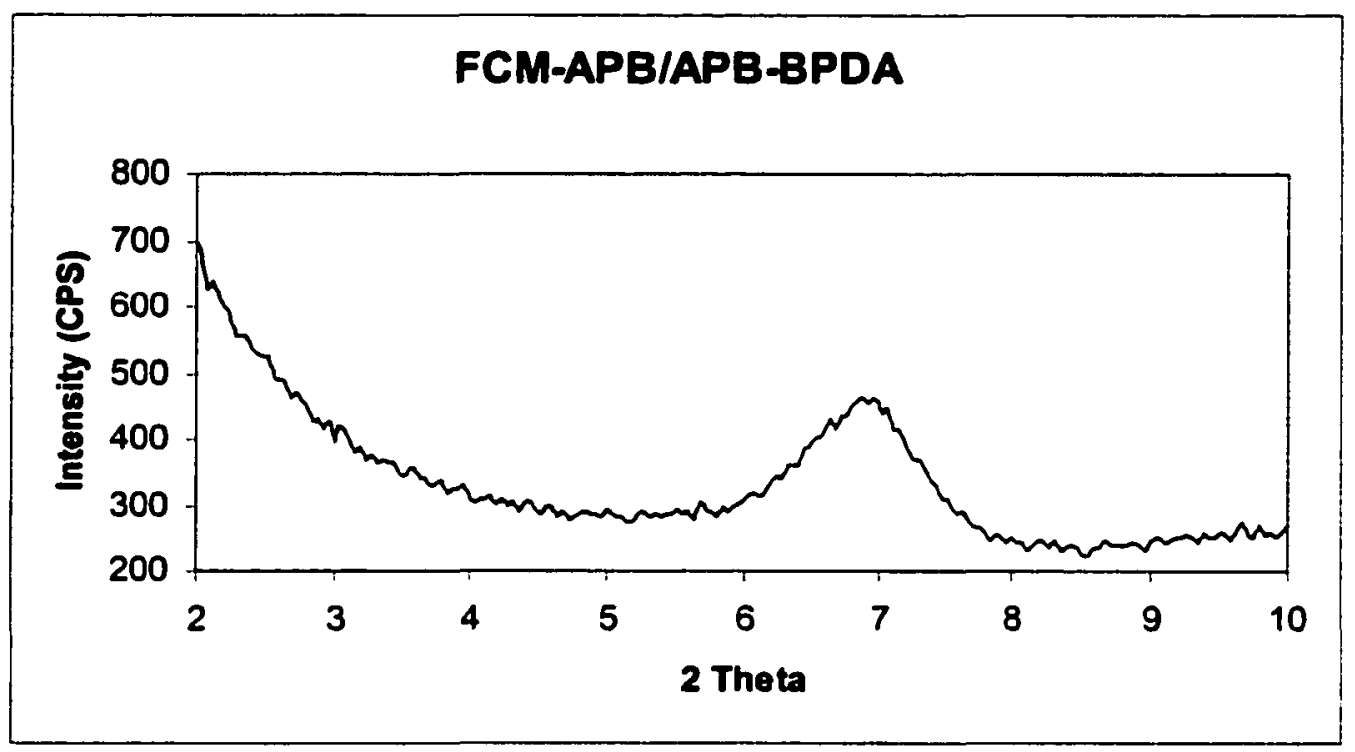

Figure 10. XRD pattern of FCM-APB/APB-BPDA nanocomposites

The XRD pattern showed a peak at $2 \theta=6.9^{\circ}$ indicating that there was some clay that had not exfoliated. At first glance the clarity of the film seemed to indicate very good dispersion but the XRD pattern showed that there was clay that had not been exfoliated.

Another attempt to exfoliate the FCM-APB organoclay involved changing the sequence of monomer addition. The same FCM-APB organoclay was used in this in-situ polymerization but in this polymerization a small amount of dianhydride was added before introducing the diamine. The amount of dianhydride added corresponded to the calculated number of surfactant amine groups available to react with the dianhydride. The final step was to add enough dianhydride to complete a 1:1 diamine:dianhydride stoichiometric polymerization reaction. The mixture and the film in this case looked very 
similar to those of the preceding FCM-APB preparation. The film was characterized via $\mathrm{XRD}$ and the pattern obtained is shown in Figure 11.

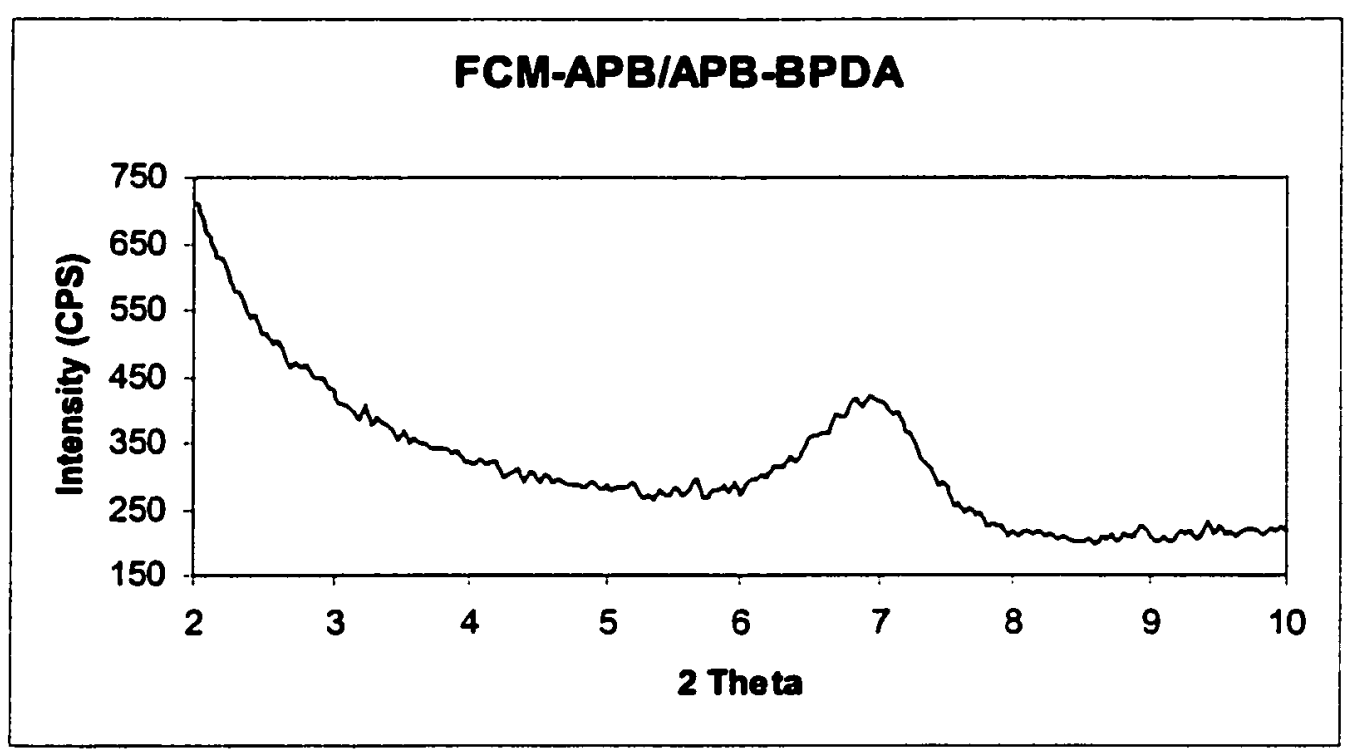

Figure 11. XRD pattern of FCM-APB/APB-BPDA hybrid film

The XRD pattern shown here was almost identical to that in Figure 10, suggesting that there is no advantage to adding small amounts of dianhydride prior to diamine addition. The films prepared with the FCM-APB organoclay showed significant improvement in visual clarity indicating that there was good dispersion of the clay particles in these films but the XRD patterns indicated that the clay was not exfoliated.

\subsection{Characterization of RCM-APB/APB-BPDA hybrids}

The RCM-250-APB organoclay was also used in the preparation of polyimide/organoclay hybrid materials. This organoclay was prepared in exactly the same manner as the FCM-APB organoclay but RCM-250-Na+ was used as the base clay. The organoclay had a reddish tint to it after drying and when placed in NMP and sonicated the suspension was reddish brown. The APB-BPDA polyimide was prepared 
in the presence of this clay but the results were different than in the FCM-APB/APBBPDA system. The hybrid mixture and the polyimide film was not as clear as the FCMAPB/APB-BPDA film and received a visual rating of 4 . The XRD pattem shown in Figure 12 shows no scattering peak, indicating exfoliation.

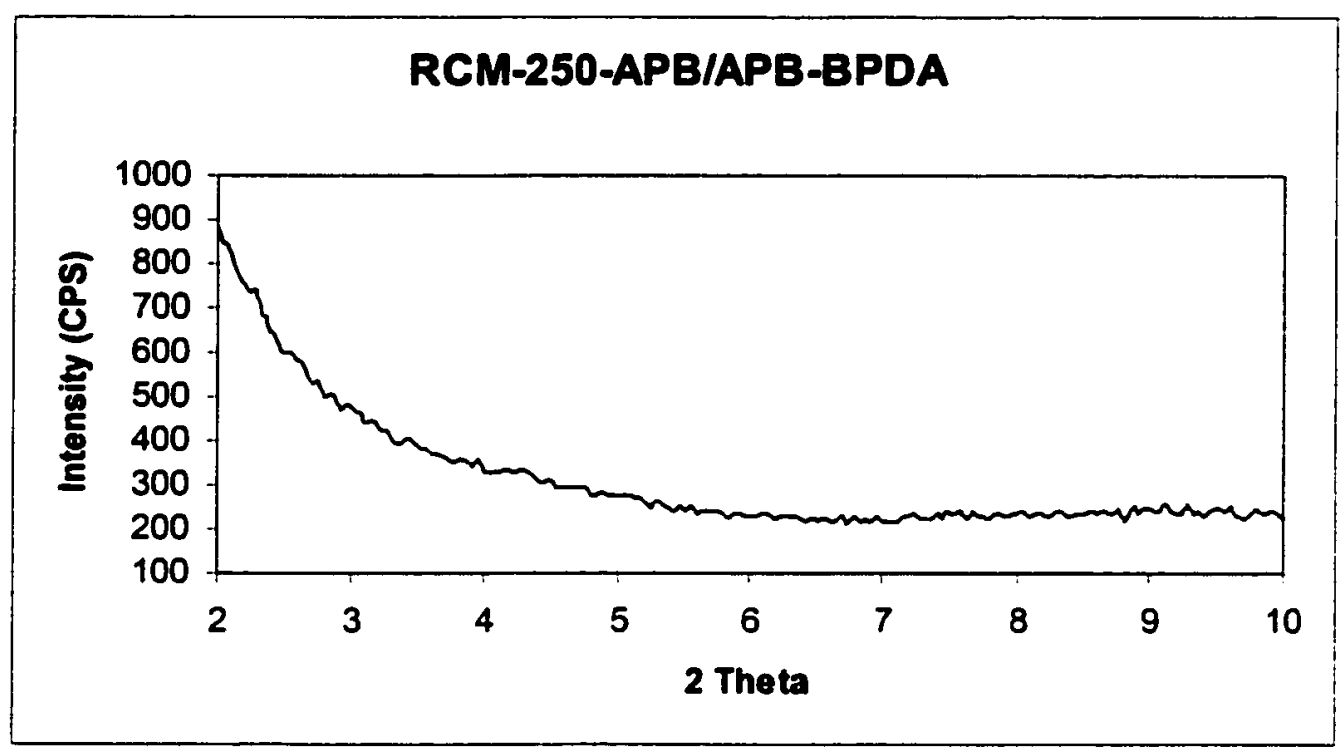

Figure 12. XRD pattern of RCM-250-APB/APB-BPDA polyimide

However, the visual analysis of the film indicated that exfoliation had not taken place so the XRD pattern is misleading. The RCM-APB organoclay was not an effective organoclay for polyimide nanocomposite preparation.

\subsection{Characterization of FCM-TBPA nanocomposites}

Nanocomposites were prepared from organoclays with various aromatic organic surfactant modifiers. To determine if aromatic surfactants would perform better in polyimides when small aliphatic chains were placed on the amine, organoclays were prepared with TBPA. The organoclay was prepared from Cloisite-Na+ through an ion exchange reaction in water. The XRD pattern of this organoclay was strange in that it 
had a peak at $2 \theta=6.68^{\circ}$ indicating a d-spacing of $1.32 \mathrm{~nm}$. This is almost in the same position as the sodium clay $(1.33 \mathrm{~nm})$. The clay was assumed to have exchanged with the TBPA even though it did not have an increase in d-spacing. The clay was used in the preparation of nanocomposite films with the APB-BPDA polymer. The mixture was yellow and cloudy after mixing for $24 \mathrm{hrs}$. A film was prepared from this mixture. The film was characterized with XRD and the XRD pattern of this film is shown below in Figure 13.

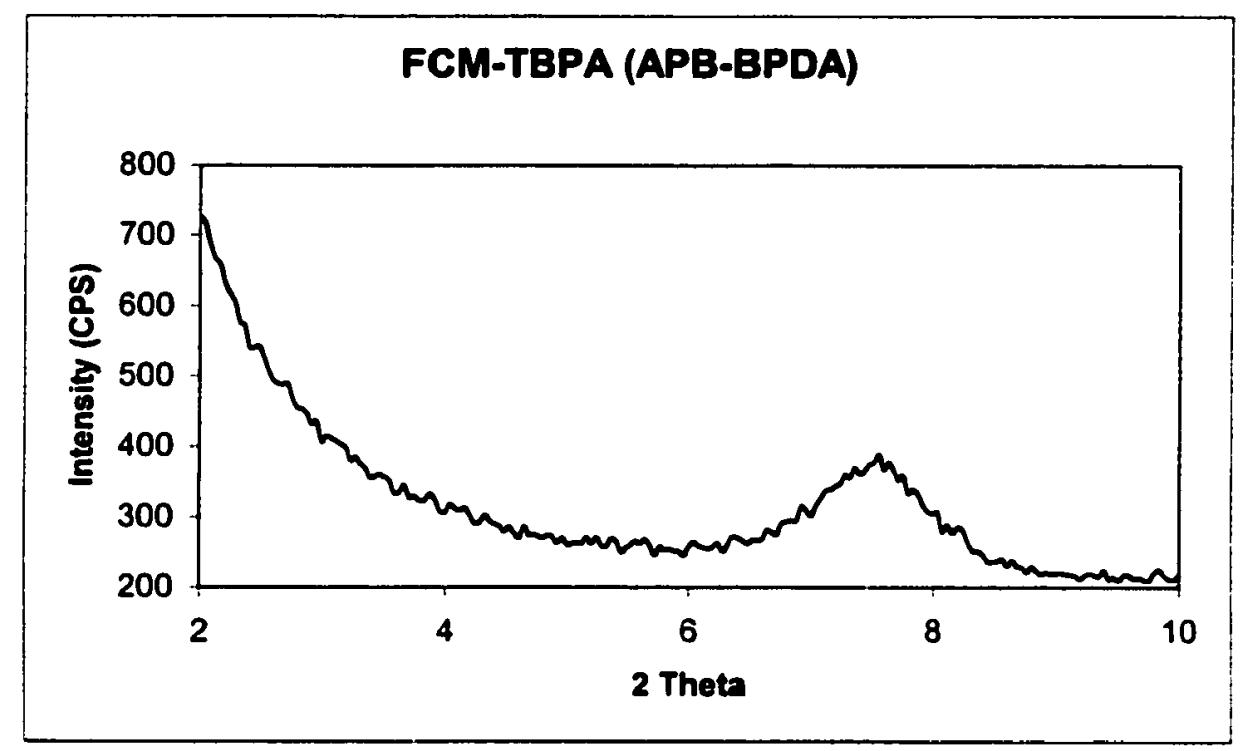

Figure 13. XRD pattern of FCM-TBPA/APB-BPDA hybrid films

The film prepared with this mixture was relatively clear with no visual clay agglomerates present and was given a visual rating of 2 . Although the film appeared to have well dispersed clay by visual appearance the XRD pattern had a peak at $2 \theta=7.56^{\circ}$. This showed that some organoclay did not exfoliate and some clay actually collapsed after the mixture was dried and cured. The concentration of collapsed particles could not be determined from this pattern and TEM micrographs were not obtained. Although the organoclay was not completely dispersed, this organoclay/polyimide combination did 
give some indication that aromatic organoclays could be used in nanocomposite preparation.

\subsection{Characterization of FCM-TMPA/APB-BPDA nanocomposites}

A host of aromatic ions were used to prepare organoclays. The simplest of these ions was the TMPA ion and consisted of only one benzene ring and three methyl groups on the nitrogen. This ion was used as the control or point of reference in these experiments, which meant that all the modifications that were done to the ion were compared with TMPA. The FCM-TMPA organoclay was prepared in an aqueous media from the mixing of TMPA and Cloisite-Na+. The organoclay flocculated into large masses during the ion exchange and washing and drying yielded a white powder. The TMPA organoclay had a $\mathrm{d}$-spacing of $1.47 \mathrm{~nm}$. This represented an increase in d-spacing relative to the sodium clay and indicated that exchange had taken place. The organoclay was placed in an organic solvent and through in-situ polymerization was dispersed in APB-BPDA. The hybrid mixture was yellow and cloudy after mixing for $24 \mathrm{hrs.} \mathrm{A} \mathrm{film} \mathrm{was} \mathrm{cast} \mathrm{from} \mathrm{the}$ $3 \%$ clay mixture and after it was cured it was examined with XRD. Figure 14 is an XRD pattern obtained from examining a thin film of FCM-TMPA/APB-BPDA polyimide. 


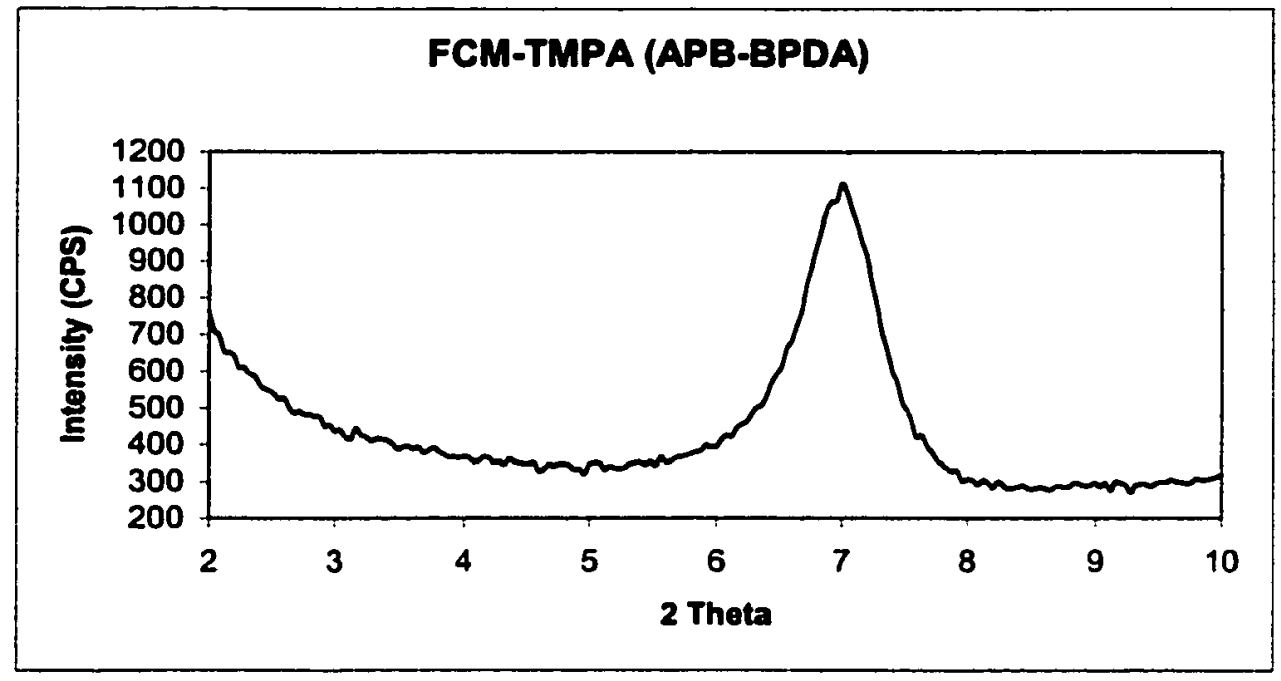

Figure 14. XRD pattern of FCM-TMPA/APB-BPDA hybrid film

At a loading level of $3 \%$ the XRD pattern of the nanocomposite film had a peak at $2 \theta=7.0^{\circ}$ which indicated that the layer spacing decreased from $1.47 \mathrm{~nm}$ for the neat organoclay to $1.26 \mathrm{~nm}$ for the film. The decrease can be accounted for by the failure of some of the clay to be impregnated by monomer or polymer and that these clay layers then collapsed during heating. While some clay may have been intercalated or exfoliated by polymer, the XRD results along with the visual appearance of the film suggest that a large amount of the clay was not affected by polymer. This combination of organoclay and polyimide was not successful in preparing nanocomposite materials.

The FCM-TMPA organoclay was also used in the preparation of hybrids with ODABTDA as the polyimide. The hybrid mixtures in this case were also cloudy. The hybrid mixtures were used to prepare films and the films were characterized. The film received a 4 for visual appearance and the XRD pattern for FCM-TMPA dispersed in ODA-BTDA is shown below in Figure 15. 


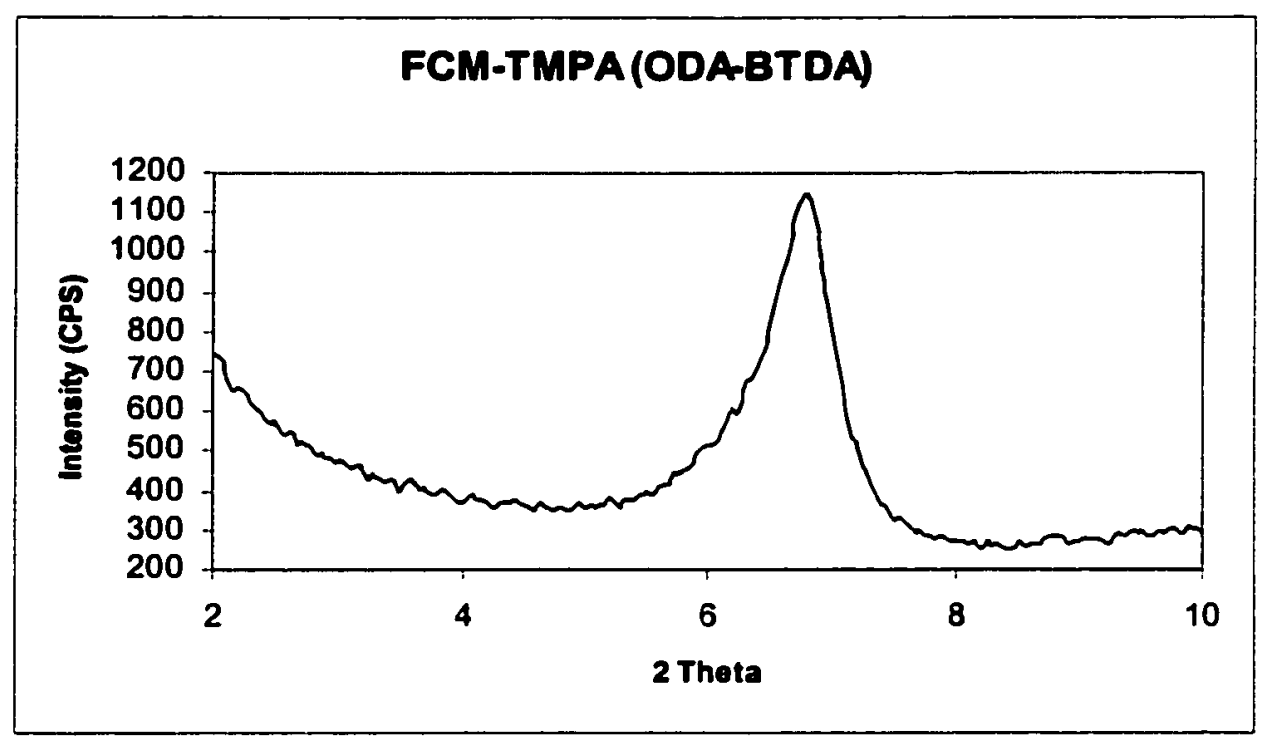

Figure 15. XRD pattern of FCM-TMPAODA-BTDA hybrid

The clay was not exfoliated. A peak with a maximum at $2 \theta=6.8^{\circ}$ in the FCM-TMPA nanocomposite corresponded to some particles in the polymer film with a d-spacing of $1.30 \mathrm{~nm}$. The clay layers that were not separated during polymerization had a smaller spacing after heating in the polyimide matrix than before combining with the PAA. This is an effect seen throughout most of the polymer systems discussed here and may be caused for different reasons. The lower temperature organoclays collapse after heating due to the degradation of the surfactant ion but these clays were assumed to be high temperature clays and should not collapse due to surfactant degradation. The amount of clay that was dispersed and the amount that was agglomerated could not be determined from these measurements.

\subsection{Characterization of RCM-250-TMPA/APB-BPDA nanocomposites}

Hybrid materials were prepared from RCM-250-TMPA organoclay to determine if lowering the CEC would facilitate clay dispersion. The organoclay was prepared in like 
manner as the FCM-TMPA organoclay. During the ion-exchange reaction the clay flocculated into a white clumps which were smaller than those formed in the FCMTMPA organoclay. The dried clay was a fine white powder and was characterized with $\mathrm{XRD}$ (Figure 16). A peak at $2 \theta=6.48^{\circ}$ corresponded to a d-spacing of $1.36 \mathrm{~nm}$. This is smaller than the $1.47 \mathrm{~nm}$ found for the fully charged organoclay prepared with this same ion. This result is consistent with the fact that the RCM-250-Na+ clay has a smaller dspacing than the FCM-Na+.

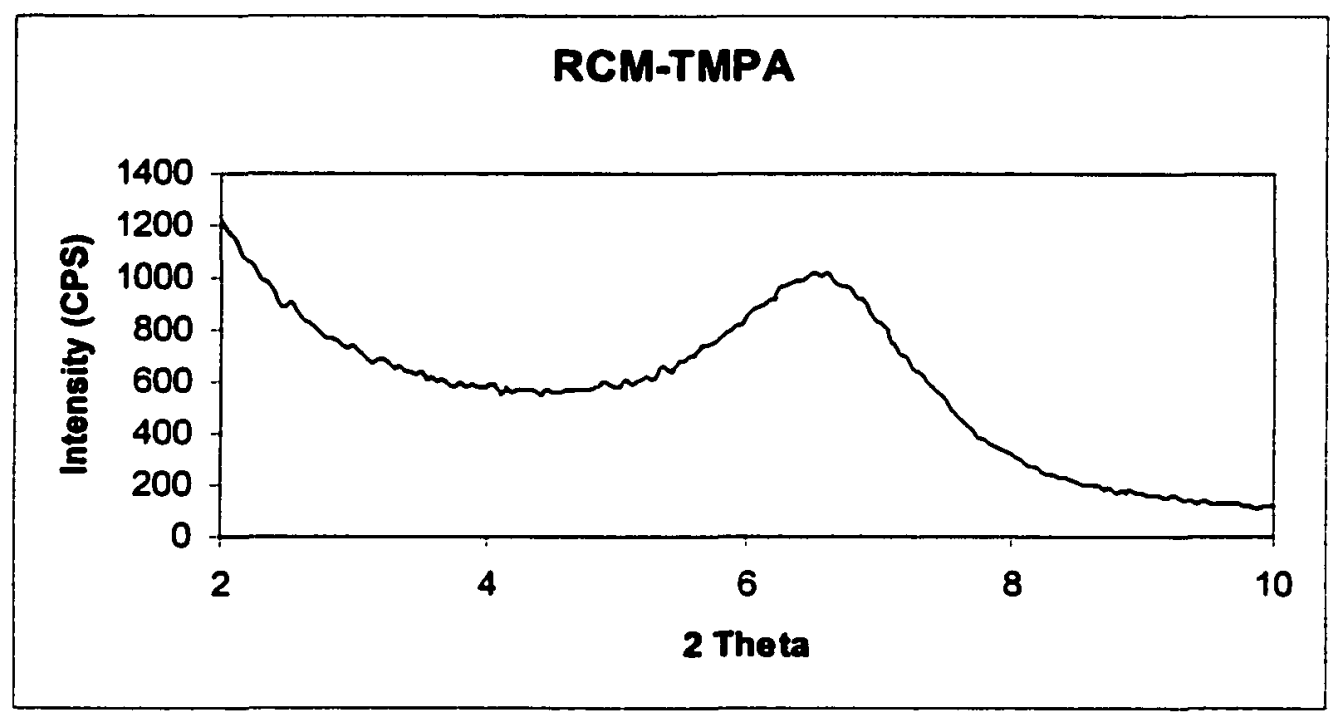

Figure 16. XRD pattern of RCM-250-TMPA organoclay

PAAVorganoclay mixtures were prepared with this organoclay and APB-BPDA polyimide. The mixture was cloudy after the in-situ polymerization. Films were prepared from these hybrid mixtures. Visual inspection indicated poor dispersion. There were some visual agglomerates and the film received a visual rating of 4 . The XRD pattern for this RCM-250-TMPA/APB-BPDA nanocomposite film prepared via in-situ polymerization is shown in Figure 17. 


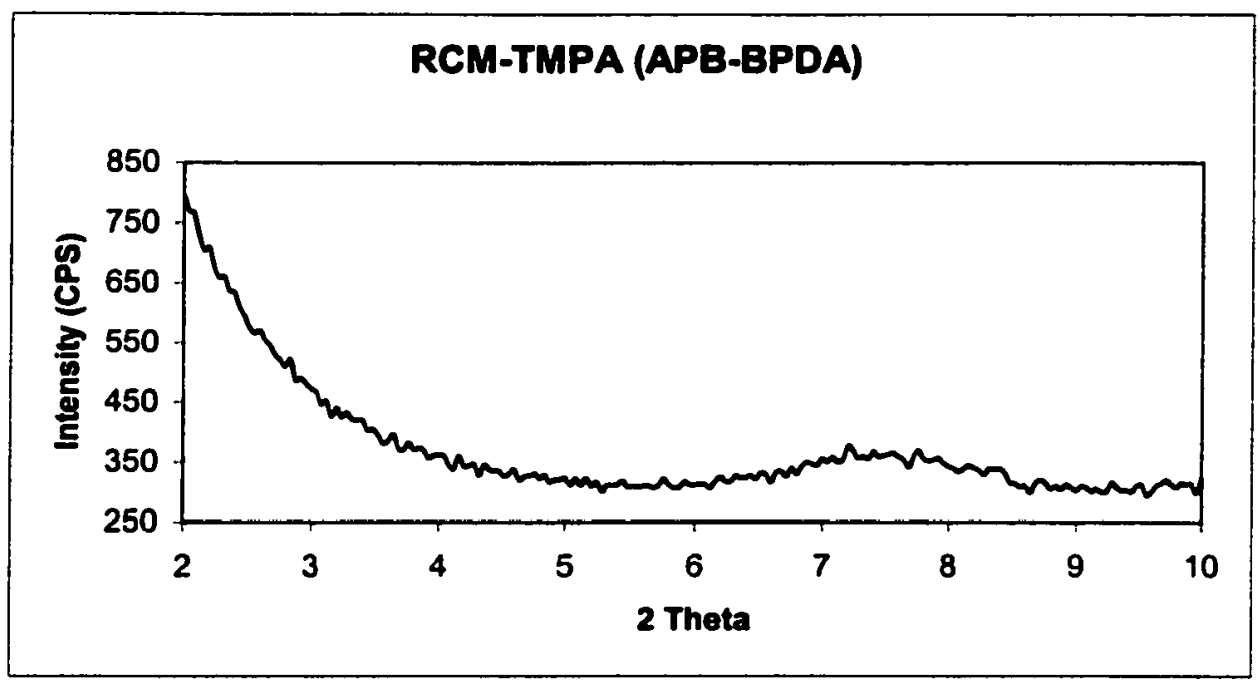

Figure 17. XRD pattem of RCM-250-TMPA/APB-BPDA hybrid film

The peak in this pattern indicates that some clay was not exfoliated. The d-spacing shifted from $1.34 \mathrm{~nm}$ prior to mixing with polymer to $1.23 \mathrm{~nm}$ after in-situ polymerization and thermal cure. TEM micrographs and other characterization of these samples are needed to more accurately assess the dispersion characteristics of this organoclay in this film.

The same RCM-250-TMPA was also used in hybrid formation with ODA-BTDA PAA. The hybrid mixture was prepared at a clay loading of $3 \%$ using in-situ polymerization. It was cloudy after PAA formation indicating that the clay was not dispersed to nanoscopic dimensions. After thermal cure, the resulting polyimide film received a visual rating of 4 . The film was characterized with XRD as shown in Figure 18. The peak indicated a d-spacing of $1.22 \mathrm{~nm}$ in the RCM-250-TMPA/ODA-BTDA film which was smaller than the FCM-TMPA clay in the same polyimide. This smaller d-spacing was not necessarily a result of poorer dispersion but was more a function of the RCM-250-TMPA organoclay which had a smaller d-spacing before mixing with the polymer than its FCM counterparts. If the clay layers were not separated during 
polymerization in both systems it is logical that RCM clay would have a smaller spacing after mixing with the polymer. It was concluded that the surfactant was not useful in compatibilizing the clay with the polyimides.

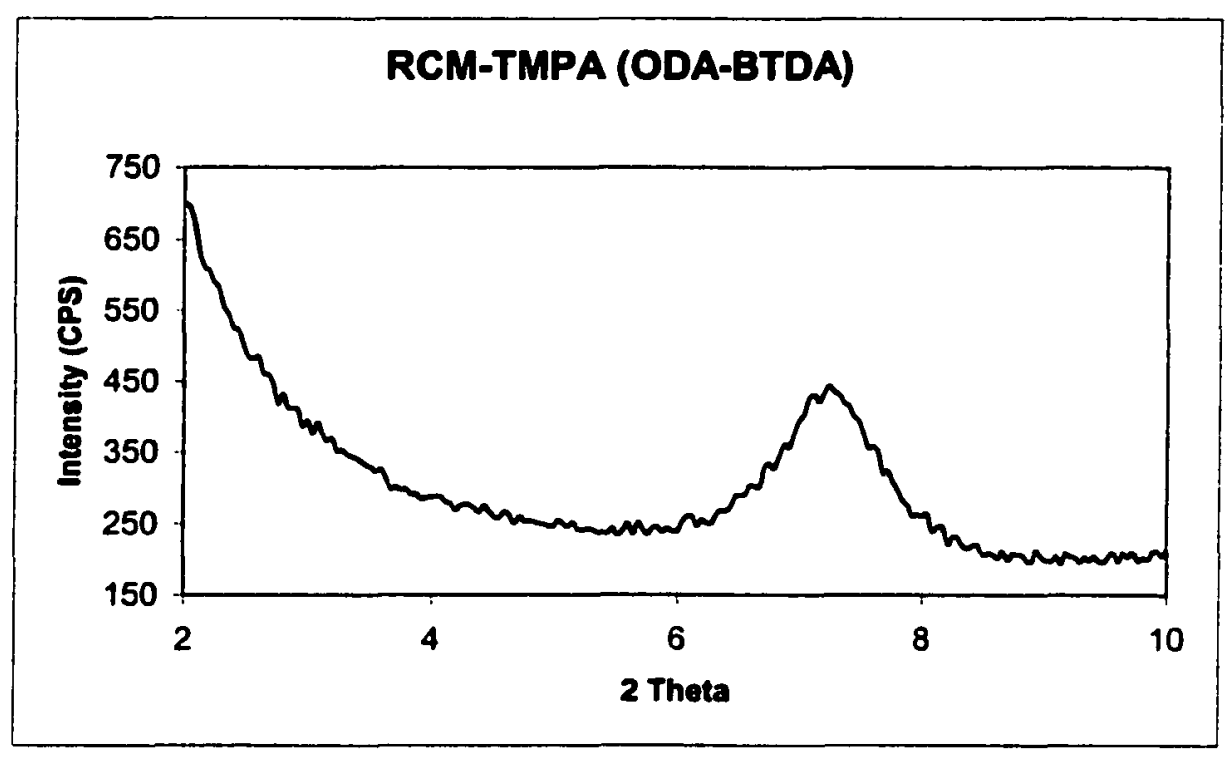

Figure 18. XRD pattern of RCM-250-TMPAODA-BTDA hybrid

\subsection{Characterization of FCM-BP nanocomposites}

Organoclays were prepared from fully charged montmorillonite and the BP ion. The organoclay was prepared in water via ion exchange and after the ion exchange reaction was completed the clay was washed and dried yielding a white powder. The powder was characterized with XRD and the d-spacing was determined to be $1.51 \mathrm{~nm}$ (see Table 3). The clay was used in the preparation of hybrid films with both APB-BPDA and ODABTDA polyimides. The nanocomposite mixtures and films were again prepared by an insitu approach at a clay loading level of 3\%. When APB-BPDA PAA was prepared in the presence of the FCM-BP organoclay, the mixture was cloudy. The FCM-BP/APB-BPDA polyimide film was assigned a visual rating of 4 . This rating indicated that the clay was 
not well dispersed. The film was also characterized with XRD and the results are shown in Figure 19.

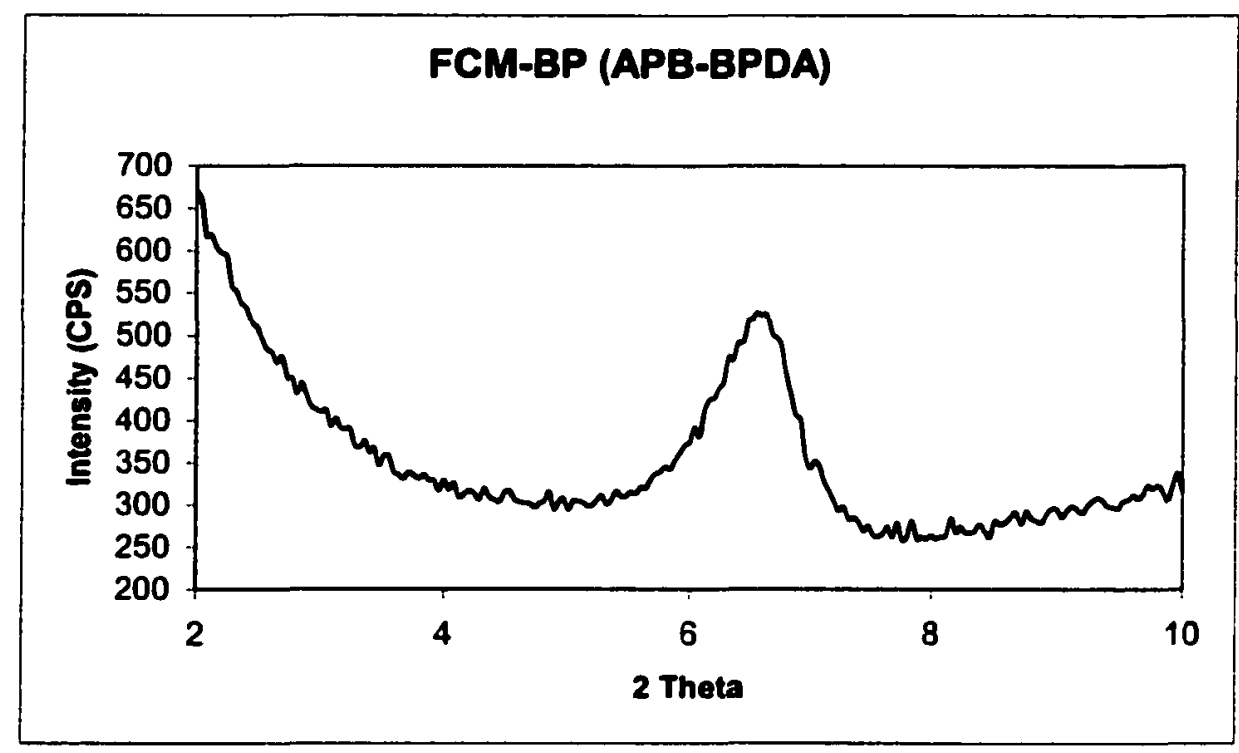

Figure 19. XRD pattern of FCM-BP APB-BPDA hybrid film

The pattern in Figure 19 shows a peak at $2 \theta=6.68^{\circ}$ and again the layer spacing decreased after hybrid formation with APB-BPDA PAA followed by conversion to the imide. This collapse was similar to the results found in the TMPA organoclays. The XRD data along with the visual inspection indicated that the clay was not well dispersed. To further investigate the dispersion TEM micrographs of the materials were obtained. The micrographs are displayed in Figure 20. 

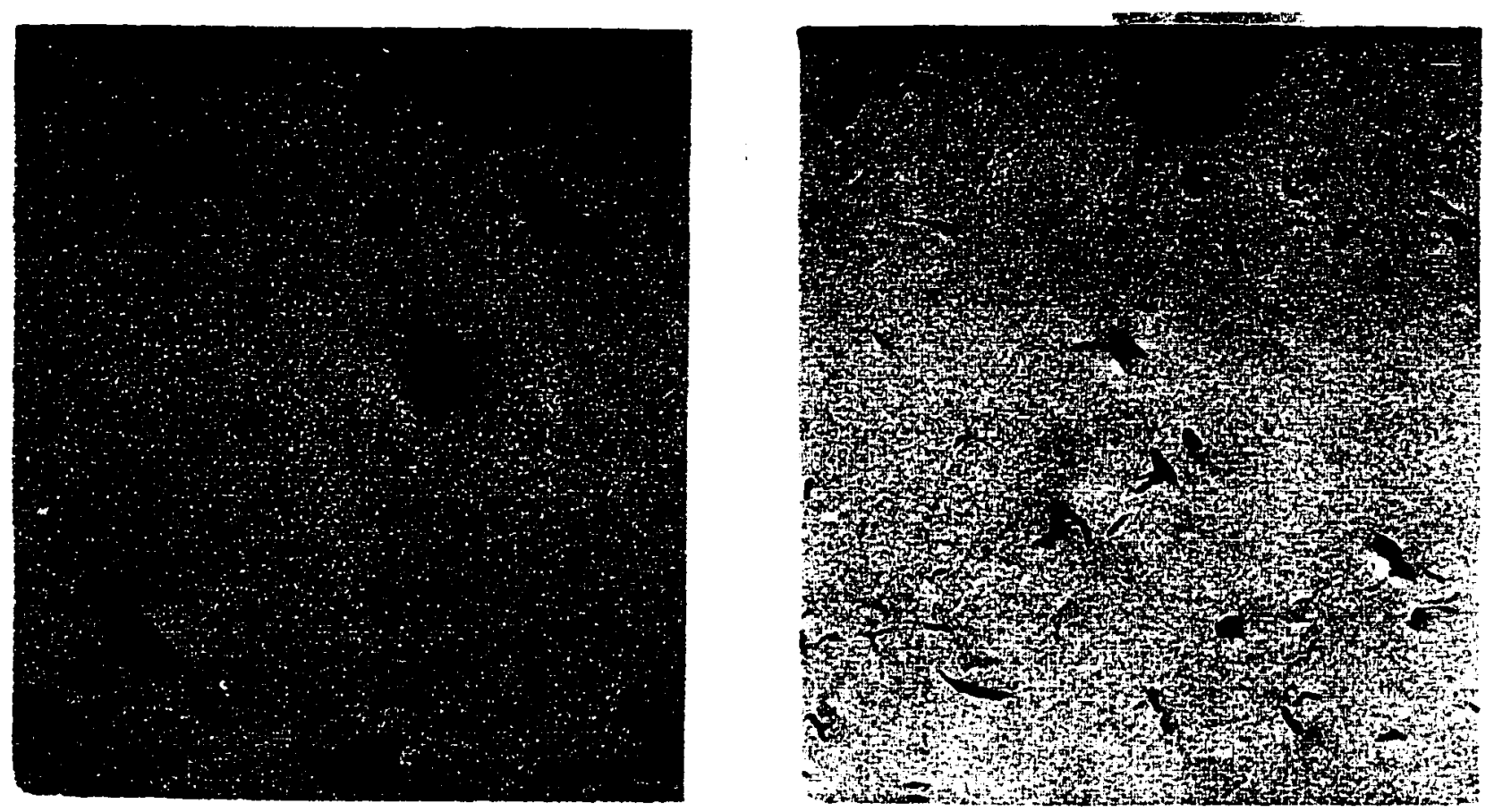

Figure 20. TEM micrograph of FCM-BP/APB-BPDA hybrid film

The micrograph in Figure 20 shows a FCM-BP/APB-BPDA hybrid film at two different magnifications (22000x and 3400x). The TEM results also indicated that the clay was not well dispersed. In the higher magnification micrograph on the left especially, the clay particles appear agglomerated. The lower magnification micrograph shows large areas where no clay particles are present. Most of the clay particles are still in stacks. These micrographs give additional evidence that there was poor dispersion of this organoclay in APB-BPDA.

FCM-BP organoclays were also examined to determine if they would disperse in ODA-BTDA polyimide. The $3 \%$ PAA/organoclay mixtures were cloudy and the film prepared from this mixture received a visual rating of 4 . The visual data as well as the XRD data indicated that the FCM-BP did not exfoliated in this polymer. The XRD 
pattern of the nanocomposite prepared from this polyimide/organoclay is shown here in Figure 21. There is a peak with a maximum at $2 \theta=6.92^{\circ}(1.28 \mathrm{~nm})$. This is smaller than the $1.51 \mathrm{~nm} \mathrm{~d}$-spacing for the organoclay by itself. This showed the same collapsing behavior of $\mathrm{d}$-spacing on curing as in the previous samples.

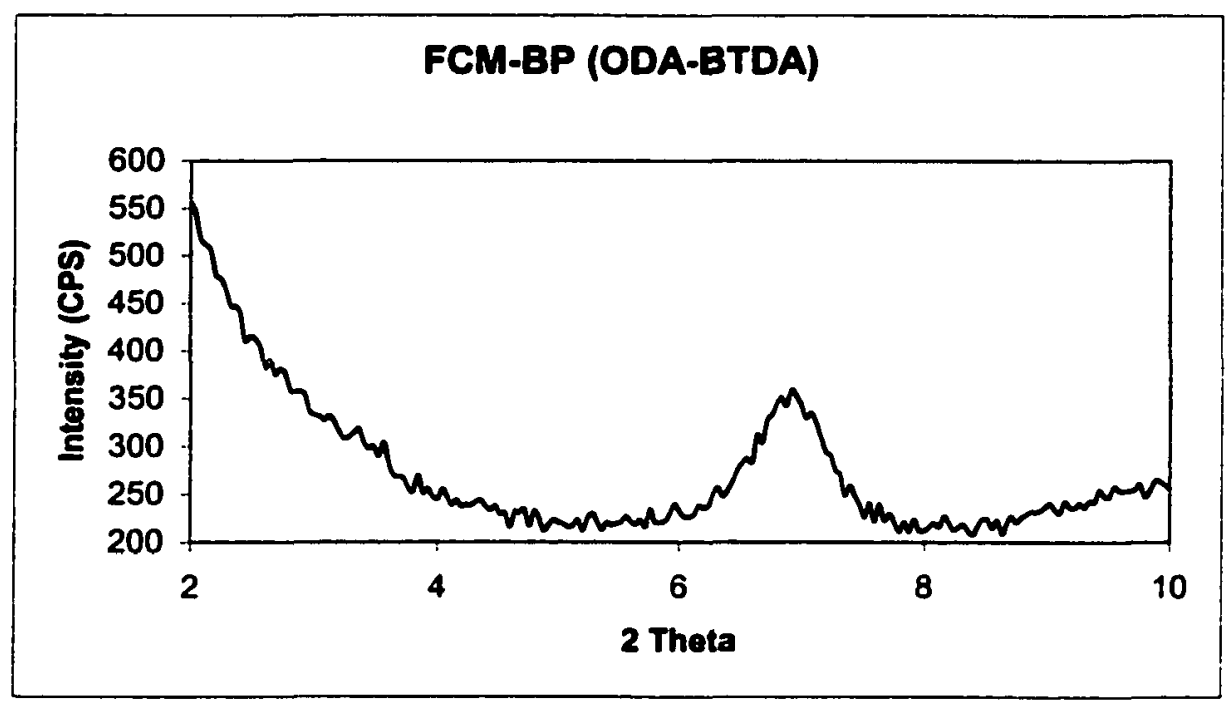

Figure 21. XRD pattern of FCM-BP/ODA-BTDA film

\subsection{Characterization of RCM-250-BP/APB-BPDA nanocomposites}

Nanocomposites were prepared with RCM-250-BP organoclays and APB-BPDA polyimide. The organoclay was prepared by replacing the sodium ion in RCM-250-Na+ with the BP ion in water. The XRD pattern obtained from the powdered sample is shown in Figure 22. There is a sharp peak centered at $2 \theta=5.8^{\circ}(1.52 \mathrm{~nm})$. This d-spacing is similar to the d-spacing of FCM-BP organoclay and is slightly larger than that of the RCM-250-TMPA. The extra phenyl ring apparently forces the clay to expand a little more than in the case of the RCM-250-TMPA. This again implies that the phenyl rings were not flat against the clay surface. 


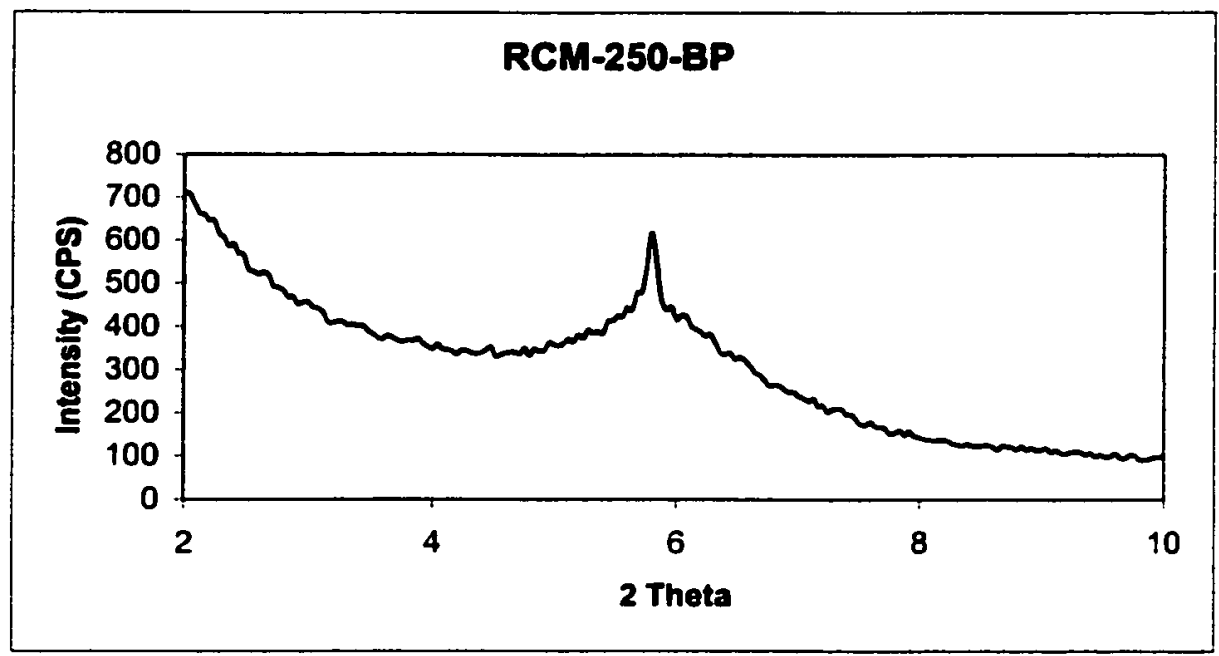

Figure 22. XRD pattern of RCM-250-BP organoclay

The clays were dispersed in NMP using sonication before an in-situ polymerization at $3 \%$ clay loading. The hybrid mixture prepared with this combination was not clear and the polyimide film was given a visual rating of 4 . Again the clay was not well dispersed. The hybrid film prepared with RCM-250-BP and APB-BPDA was examined with XRD and the pattern is shown below in Figure 23.

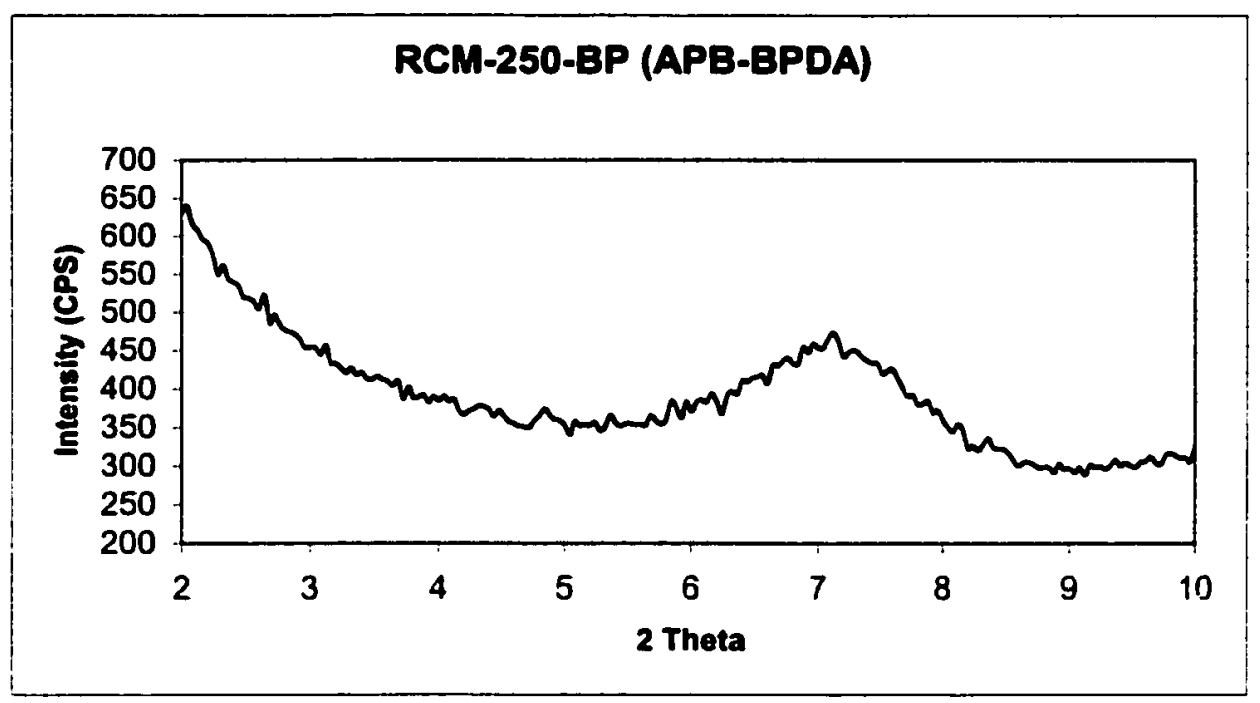

Figure 23. XRD pattern of RCM-250-BP/APB-BPDA hybrid film 
The peak maximum was at $2 \theta=7.12^{\circ}$ indicating a d-spacing of $1.24 \mathrm{~nm}$. This d-spacing is smaller than the d-spacing (1.35) of the clay in the FCM-BP/APB-BPDA nanocomposite. The FCM-BP/APB-BPDA and the RCM-250-BP/APB-BPDA films were visually similar and the d-spacings of the clays in the polyimide were similar as well. The TEM micrographs of the film (Figure 24) showed that the clay was poorly dispersed in the RCM-250-BP/APB-BPDA film. Slightly greater dispersion was seen in this film than in the FCM-BP film (Figure 20). However, the difference was small. The conclusion that reducing the charge on the clay increased dispersion could not be made with certainty.
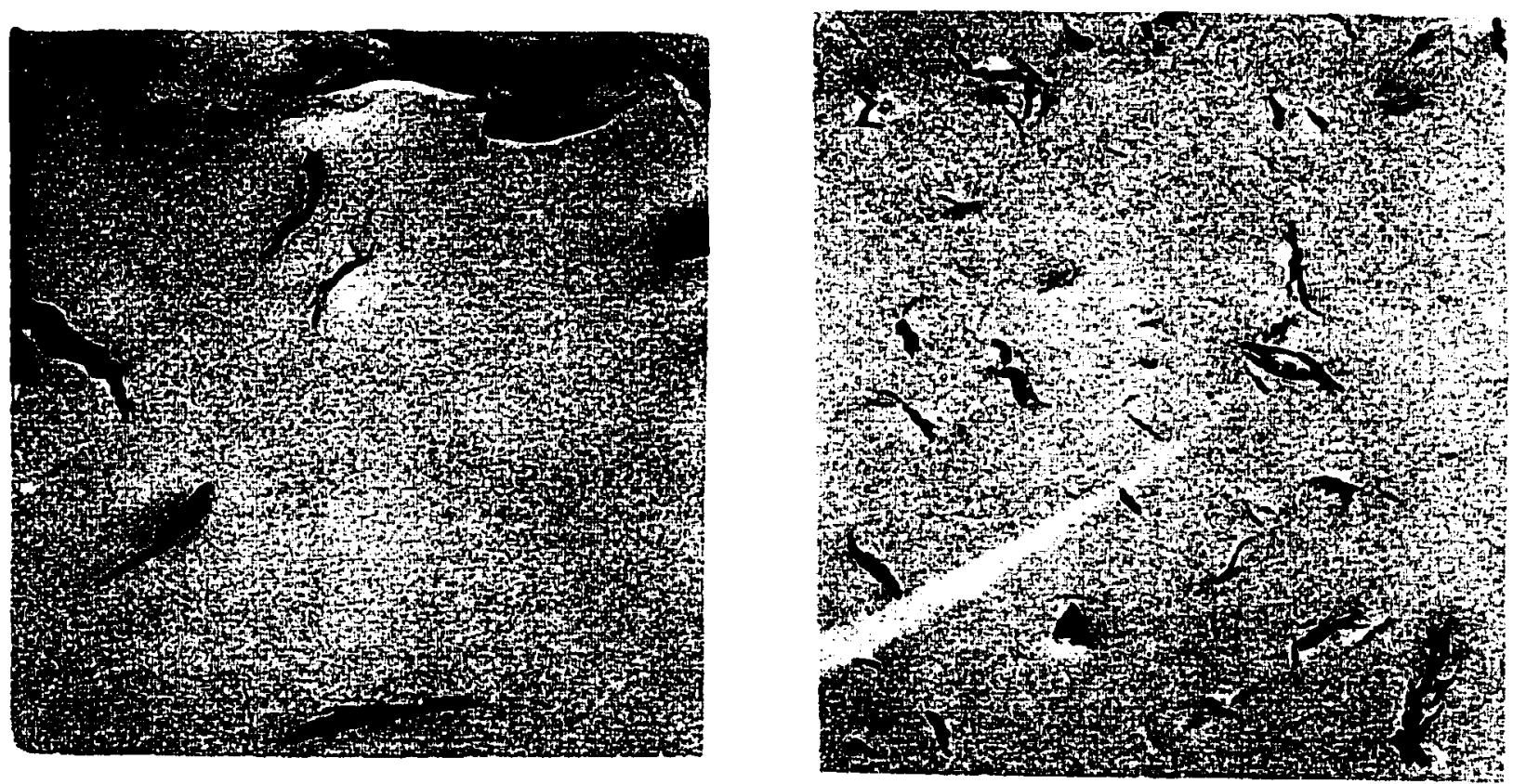

Figure 24. TEM micrograph of RCM-250-BP/APB-BPDA hybrid film

The RCM-250-BP organoclay was also used in the preparation of a hybrid mixture and a film with ODA-BTDA. The hybrid mixture was cloudy and the films prepared with this mixture received a visual rating of 4 . In Figure 25 the XRD pattern of the 
RCM-250-BP/ODA-BTDA polyimide film exhibits a peak at approximately $2 \theta=7.16^{\circ}$ $(1.23 \mathrm{~nm})$. Again this is a smaller d-spacing than the organoclay by itself $(1.52 \mathrm{~nm})$. The d-spacing is slightly smaller than the spacing $(1.28 \mathrm{~nm})$ of the FCM-BP/ODA-BTDA nanocomposite. It was hard to determine which of the organoclays modified with the BP ion is better dispersed in ODA-BTDA using the XRD data.

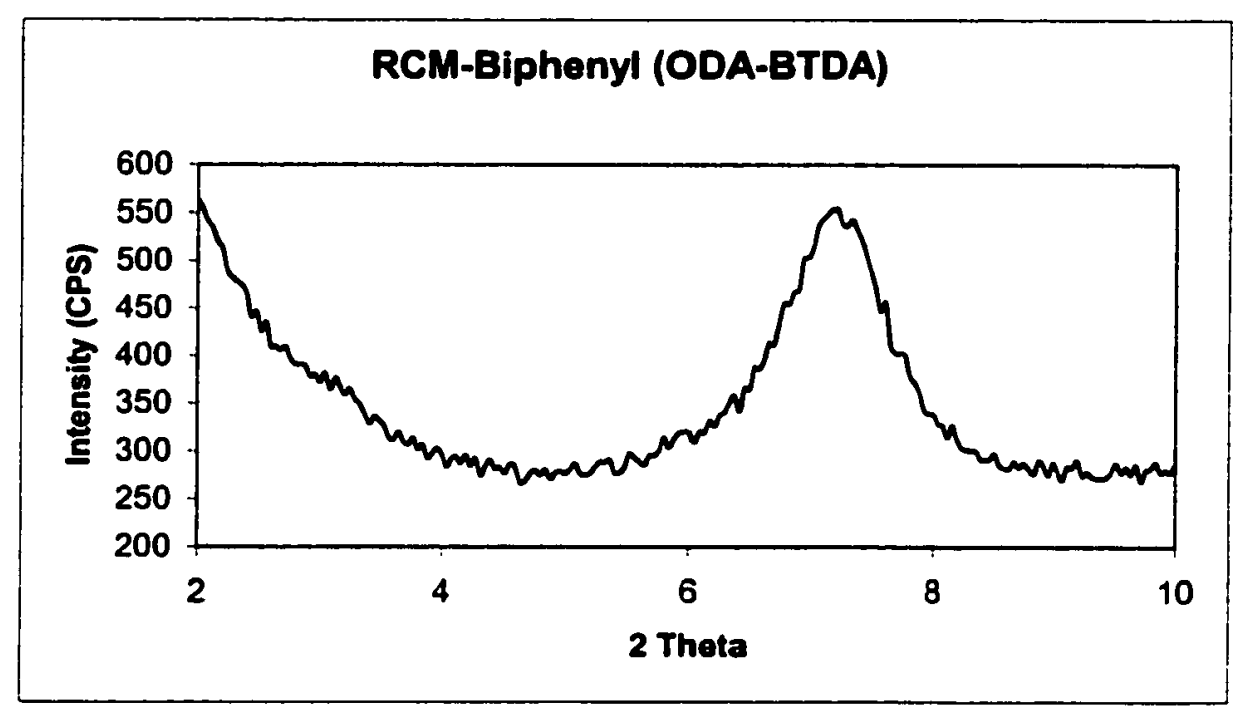

Figure 25. XRD pattern of RCM-250-BP/ODA-BTDA hybrid film

The films prepared with BP-organoclays showed that this surfactant did not modify the clay surface so as to make them useful in polyimide nanocomposite preparation. The reduction of charge may have helped a little but the overall conclusion was that all the BP-organoclays were not useful organoclays.

\subsection{Characterization of FCM-TPP nanocomposites}

In this effort to prepare organoclays that would exfoliate in polyimide matrices, more organoclays were prepared and used in nanocomposite preparation. FCM-TPP and RCM-250-TPP organoclays were examined by XRD in powder form and used in hybrid 
preparation. The results of characterization of the organoclays prepared with the TPP surfactant as well as the polyimide nanocomposite films are presented below.

FCM-TPP organoclays were prepared and used in the preparation of nanocomposite mixtures and films. The organoclay was prepared in an ion-exchange reaction of TPP with Cloisite-Na + in water. The reaction yielded a white powder after washing and drying. The organoclay had a large d-spacing at $1.74 \mathrm{~nm}$ (see Table 3). Hybrid mixture and films were prepared from this FCM-TPP organoclay via in-situ polymerization. The PAA/organoclay mixture was cloudy prior to film preparation. The cured film received a visual rating of 3 . This rating was an improvement over other films. The clay appeared to be slightly more dispersed than the organoclays in other films prepared with the other aromatic organoclays (e.g. BP, TMPA). The XRD pattern of the $3 \%$ clay loaded APBBPDA hybrid film prepared with FCM-TPP organoclay is shown in Figure 26.

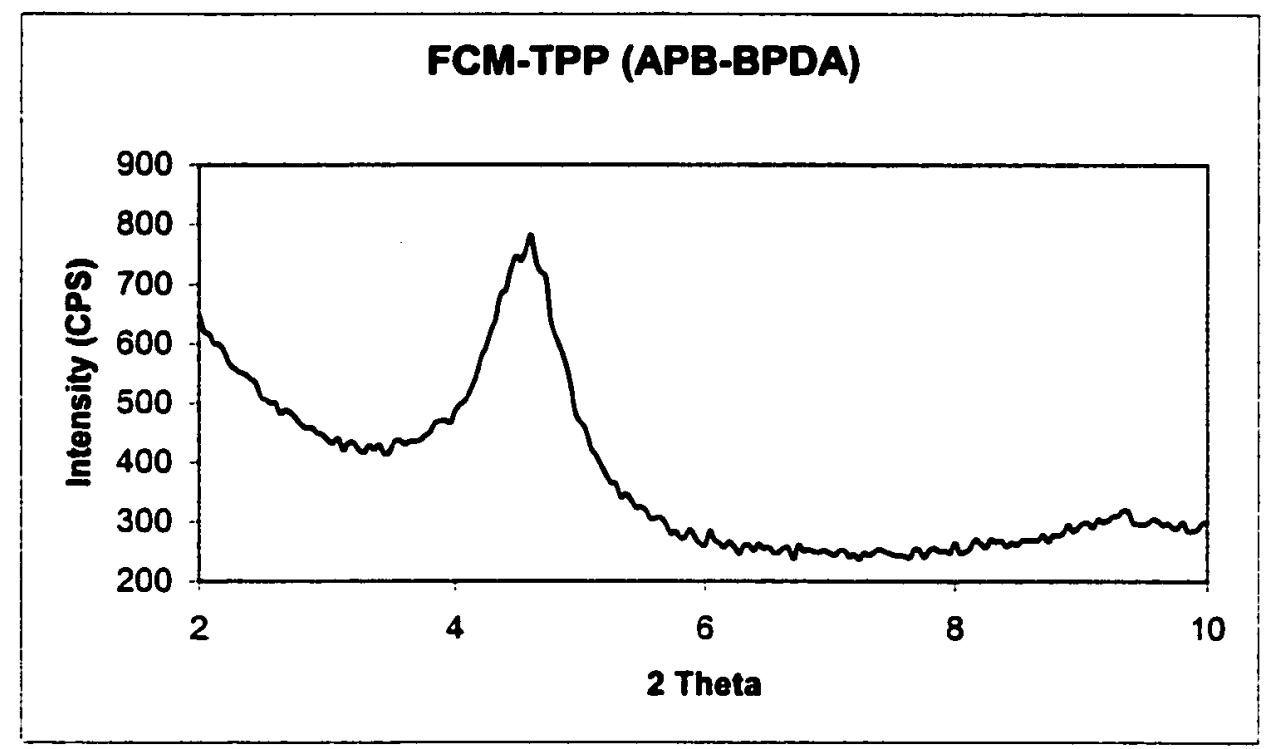

Figure 26. XRD pattern of FCM-TPP/APB-BPDA hybrid film

The peak in this XRD pattern has a maximum at $2 \theta=4.04^{\circ}$. This indicates a large $d-$ spacing of $2.19 \mathrm{~nm}$. This was the largest $\mathrm{d}$-spacing of any of the aromatic clays dispersed 
in a polymer film. The presence of this peak indicated that there was some clay in the sample that did not exfoliate. Again the concentration of agglomerated clay was not known.

The TEM results for the FCM-TPP/APB-BPDA nanocomposite films are shown in Figure 27. Again the results show that the clay is not exfoliated in this sample. Clay agglomerates are clearly present in the micrographs. This is consistent with XRD data and visual analysis of these films. The FCM-TPP organoclay is slightly better dispersed than the FCM-BP (Figure 20) as evidenced by the greater number of separate clay particles in the micrographs.
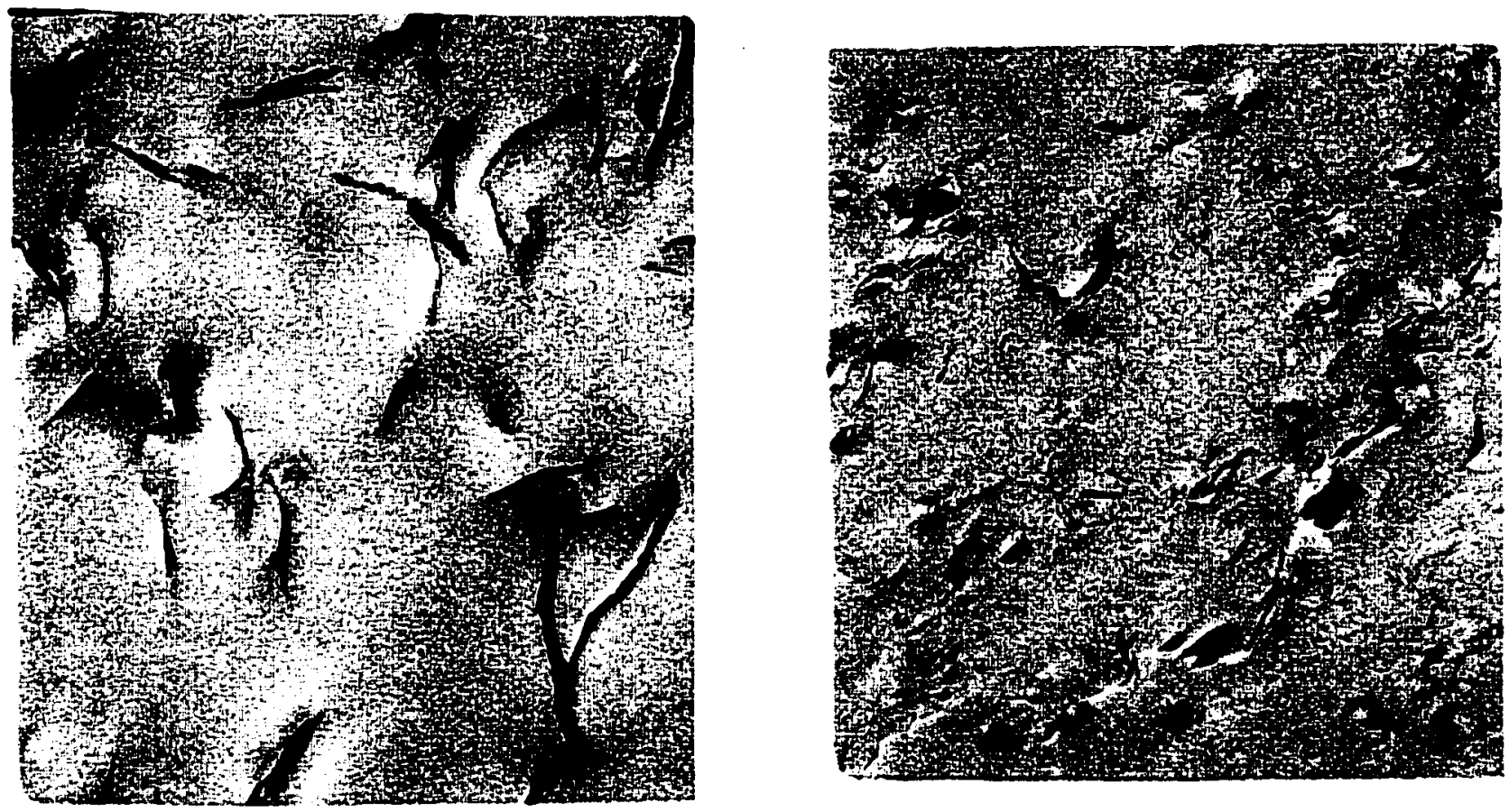

Figure 27. TEM micrograph of FCM-TPP/APB-BPDA hybrid film 
The FCM-TPP organoclay was also used in the preparation of nanocomposite material with ODA-BTDA polyimide. The PAA hybrid mixture was cloudy and the film prepared from the material received a visual rating of 4. An XRD pattern was obtained on this material and is shown in figure 28.

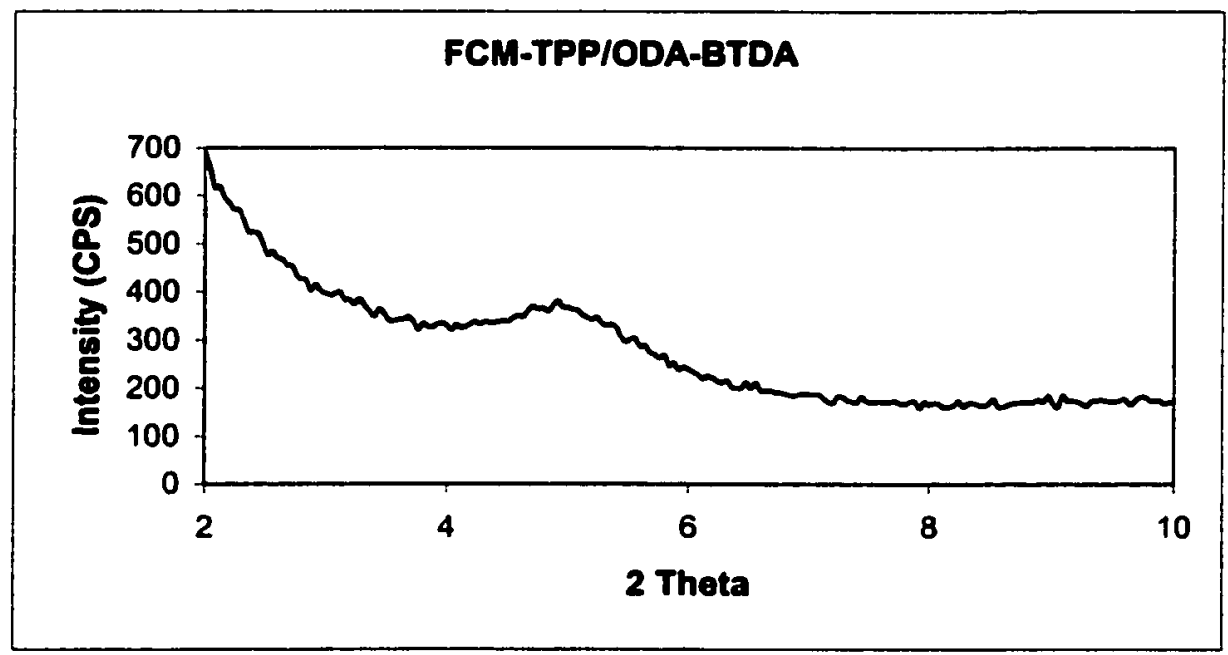

Figure 28. XRD pattern of FCM-TPP/ODA-BTDA nanocomposite

There was a peak in the XRD pattern at approximately the same angle seen in the organoclay. Although the clay did not exfoliate it appears that the clay structure did not collapse upon heating.

\subsection{Characterization of RCM-250-TPP/APB-BPDA nanocomposites}

RCM-250-TPP organoclay was prepared by ion exchange of RCM-250-Na+ with TPP in water. After washing the clay and drying the clay under vacuum at $70^{\circ} \mathrm{C}$ the clay powder was white and yielded the XRD pattern shown in Figure 29. The XRD patterns were obtained for comparison with the XRD peaks of hybrid films prepared with these clays and the XRD pattern of other reduced charge organoclays. 


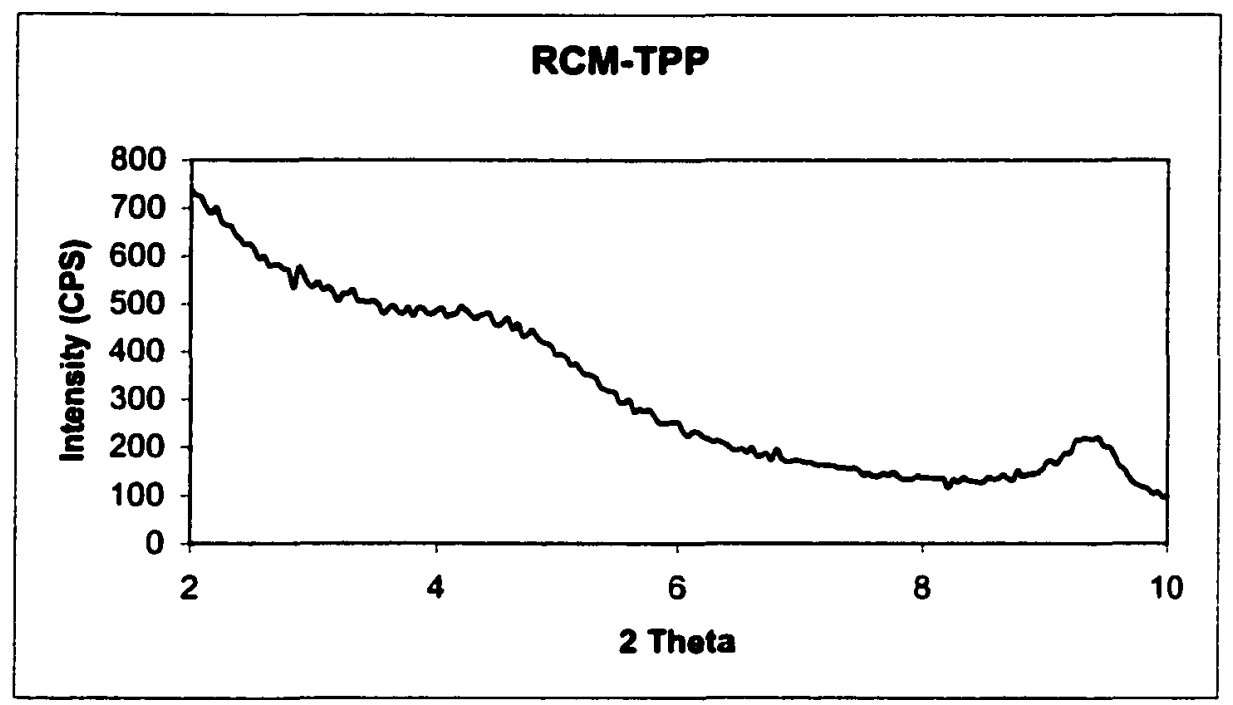

Figure 29. XRD pattern of RCM-250-TPP organoclay

Two peaks are seen in this pattern indicating two different layer spacings. The first of these is centered at $2 \theta=4.04^{\circ}(2.19 \mathrm{~nm})$ and the second is at $2 \theta=9.32^{\circ}(0.95 \mathrm{~nm})$. This indicates that not all of the clay was exchanged with the TPP. The clay that is represented by the peak at $2 \theta=9.32^{\circ}$ was not exchanged. The layer spacing of the clay exchanged with TPP is larger than the layer spacing of other organoclays prepared with aromatic ions. The clay may not have completely exchanged because of the size of the TPP ion. There simply is not enough room for this bulky ion to enter some of the smaller galleries of the RCM-250 clay. The RCM-250-TPP organoclay was used in the preparation of APB-BPDA nanocomposites. The hybrid mixture was cloudy after in-situ polymerization and was used for preparing a film. After curing the film was hazy and received a visual rating of 3 . The XRD pattern for the film was obtained and the pattern is displayed in Figure 30. 


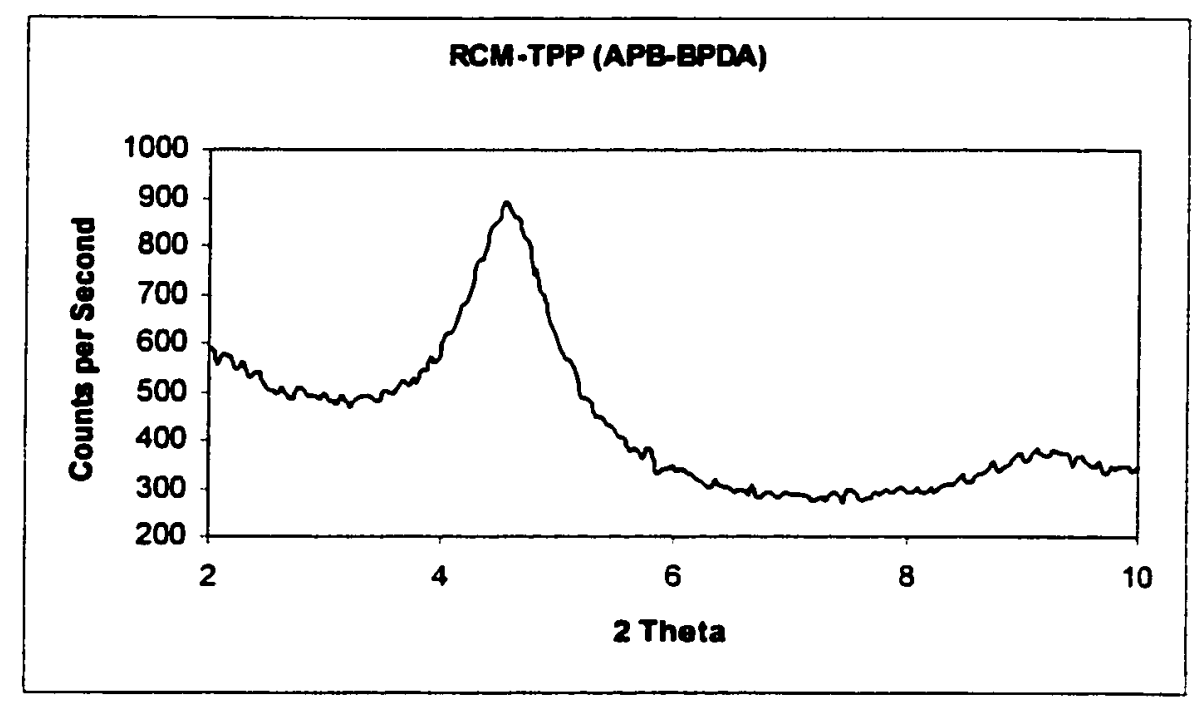

Figure 30. XRD pattern of RCM-250-TPP/APB-BPDA hybrids

The hybrid film had clay particles that were not exfoliated in the polymer matrix as indicated by two peaks in the XRD pattern at $2 \theta=4.56^{\circ}(1.94 \mathrm{~nm})$ and $2 \theta=9.36^{\circ}(0.95$ $\mathrm{nm})$. The latter is the consequence of the organoclay that had not exchanged with TPP. This XRD pattern resembles the FCM version (Figure 26). Both films received a visual rating of 3. The TEM micrographs of films prepared with RCM-250-TPP are presented in Figure 31. Again clay agglomerates are evident in the micrographs. There was some clay that was exfoliated as well. This is consistent with XRD and visual inspection. The clay in the RCM-250-TPP/APB-BPDA film may be less dispersed than in the FCM-TPP film (Figure 27). This may be a result of the fact that the RCM-250-TPP contained some clay that was not exchanged with the TPP. 

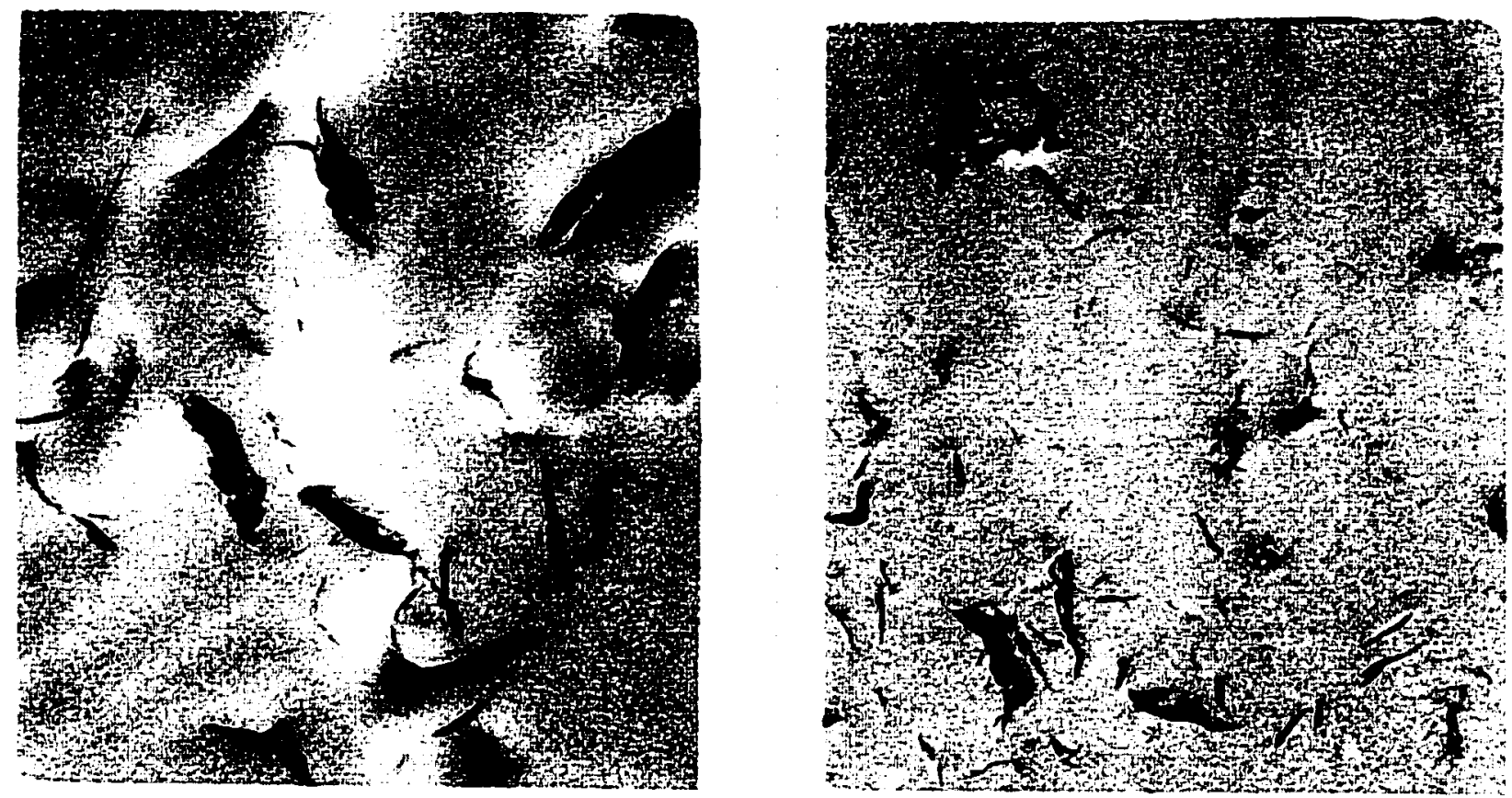

Figure 31. TEM micrograph of RCM-250-TPP/APB-BPDA hybrid film

The RCM-250-TPP organoclay was also used in the preparation of hybrids with ODA-BTDA polyimide. The 3\% PAA/organoclay mixture was prepared via in-situ polymerization and was cloudy. The clay was obviously not exfoliated. The hybrid mixture was used to pour a film. The film was cloudy as a consequence of agglomeration. It received a visual rating of 4 . The film was also characterized with $\mathrm{XRD}$ (Figure 32). 


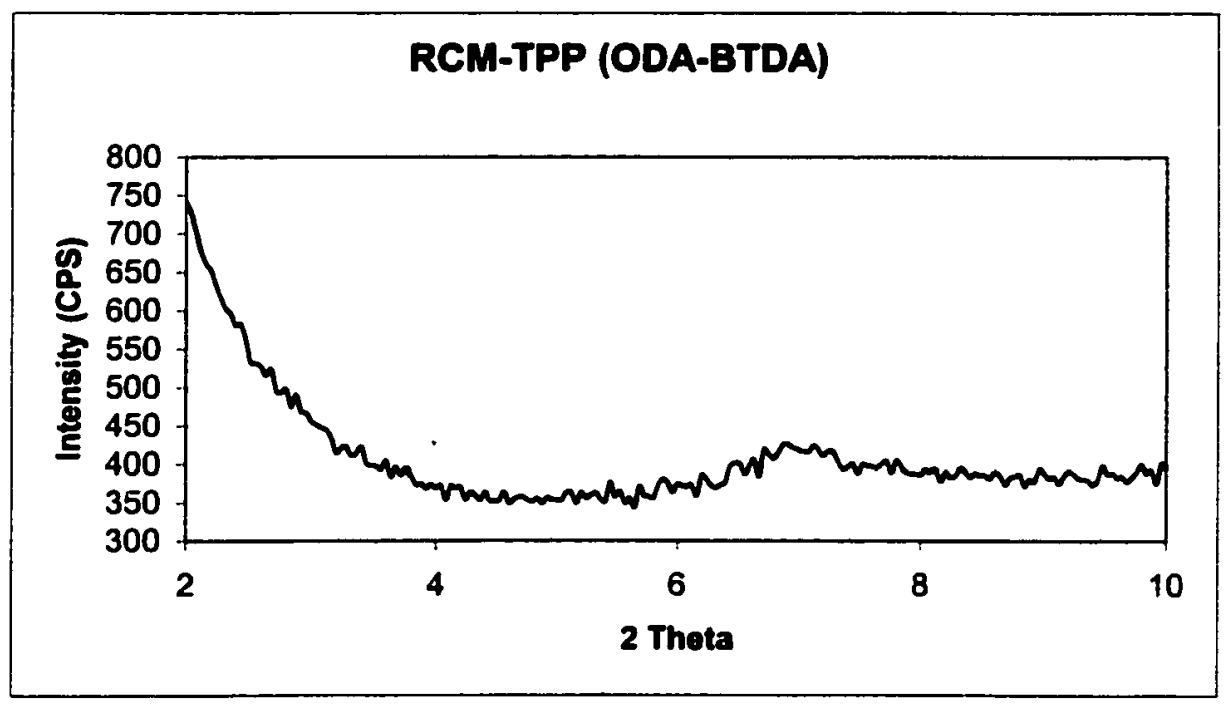

Figure 32. XRD pattern of RCM-TPP/ODA-BTDA hybrids

A peak at $2 \theta=6.92^{\circ}$ indicated that the clay that was not exfoliated and had a d-spacing of $1.28 \mathrm{~nm}$. The XRD pattern and the visual appearance of this film indicated that this organoclay/polyimide combination did not yield high quality nanocomposite materials. This was true with all the preparations where TPP was the surfactant in the organoclay.

\subsection{Characterization of FCM-Na/ODA-BTDA nanocomposites}

Charge reduction in the RCM clays allows for more space on the silicate oxide surface that the polymer can directly interact with. This space becomes available as a result of fewer ions on the surface. To determine if this extra space facilitated dispersion, ODA-BTDA PAA was prepared in the presence of the FCM-Na+ clay and the RCM-250$\mathrm{Na}+$ clay. The FCM-Na+ clay was the Cloisite-Na+ commercial organoclay. The yellow powder was examined with XRD and the pattern is shown below in Figure 33. 


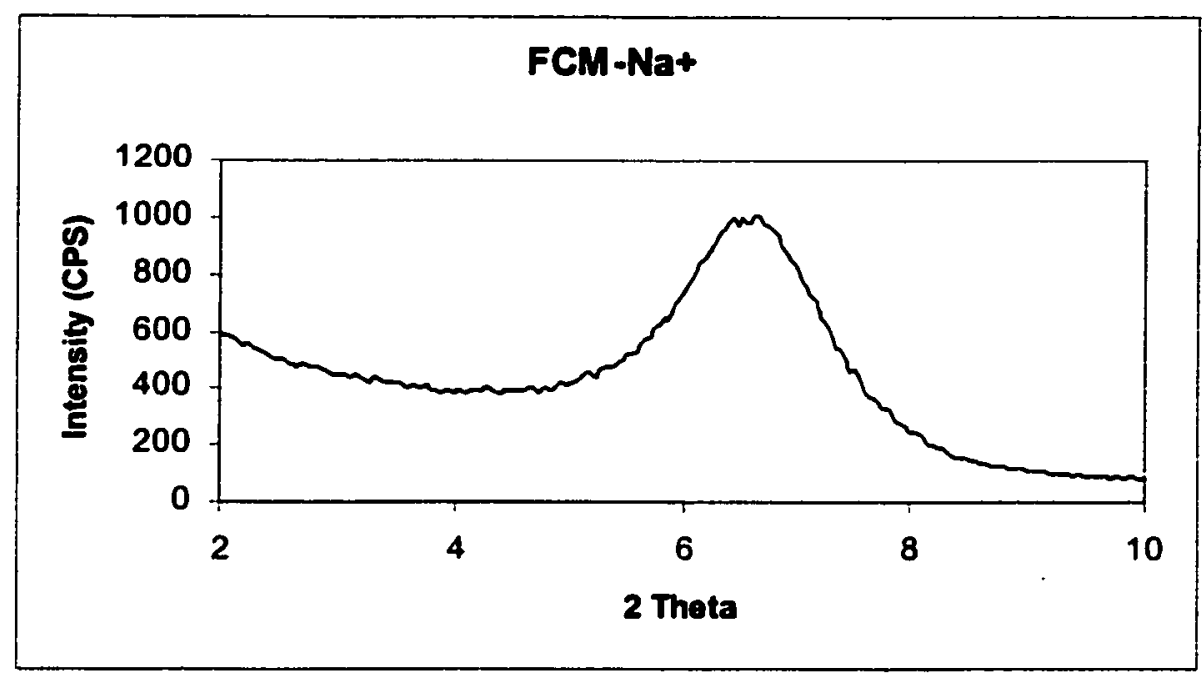

Figure 33. XRD analysis of FCM-Na clay sample

The d-spacing of this clay is $1.33 \mathrm{~nm}$ as determined by this XRD pattern. The clay was dispersed in organic solvent (NMP) and in-situ polymerization of ODA-BTDA was performed. The PAA mixture was cloudy after polymer formation and stirring for $24 \mathrm{hrs}$. The film prepared from this mixture was also cloudy and had a visual rating of 4 . The cured film was also characterized with XRD. The results in Figure 34 revealed only a small shift from $2 \theta=6.6^{\circ}$ to $2 \theta=6.92^{\circ}(1.28 \mathrm{~nm})$ when the FCM-Na+ clay was incorporated in the polyimide. The existence of a peak did indicate that at least some of the clay was not exfoliated by the in-situ polymerization of ODA-BTDA. 


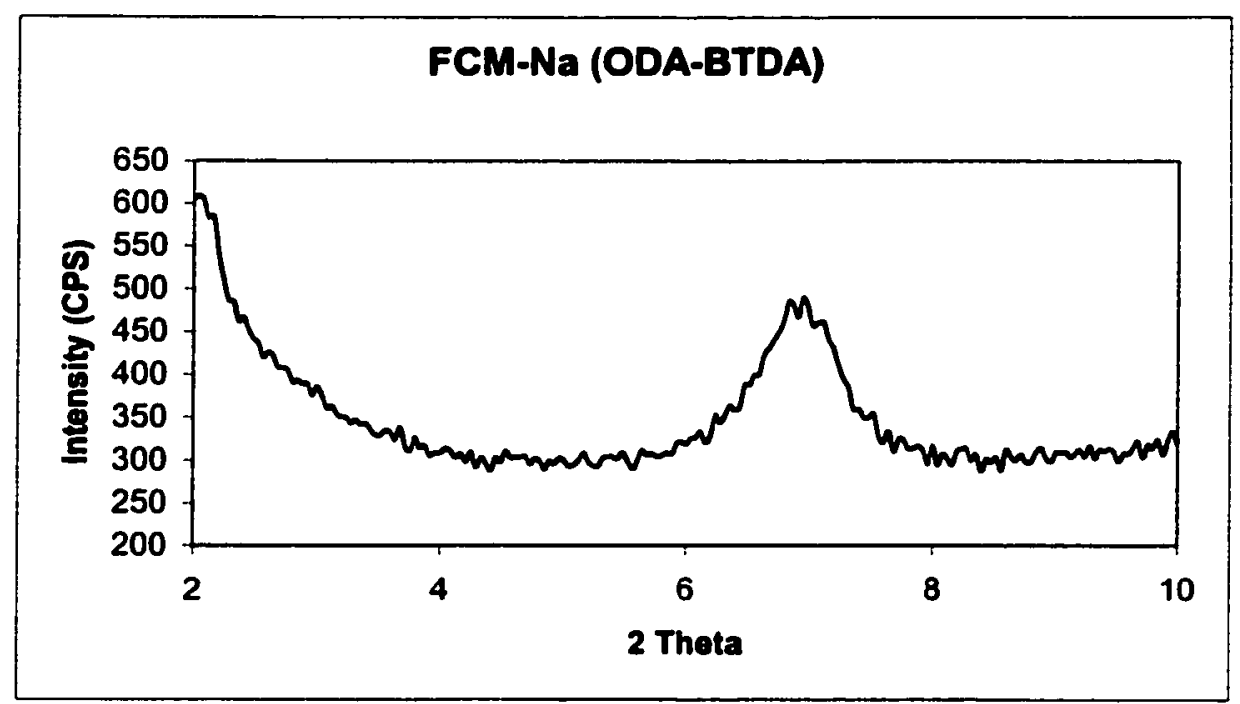

Figure 34. XRD analysis of FCM-Na/ODA-BTDA hybrids

\subsection{Characterization of RCM-250-Na+/ODA-BTDA hybrids}

Hybrid mixtures and films were prepared from the RCM-250-Na+ clay and the ODABTDA polyimide. The results have been compared to the results from Section 3.19. As shown in Figure 35 the RCM-250-Na+ clay has a d-spacing $(0.97 \mathrm{~nm})$ that is much smaller than the $1.33 \mathrm{~nm}$ of the FCM-Na+ clay.

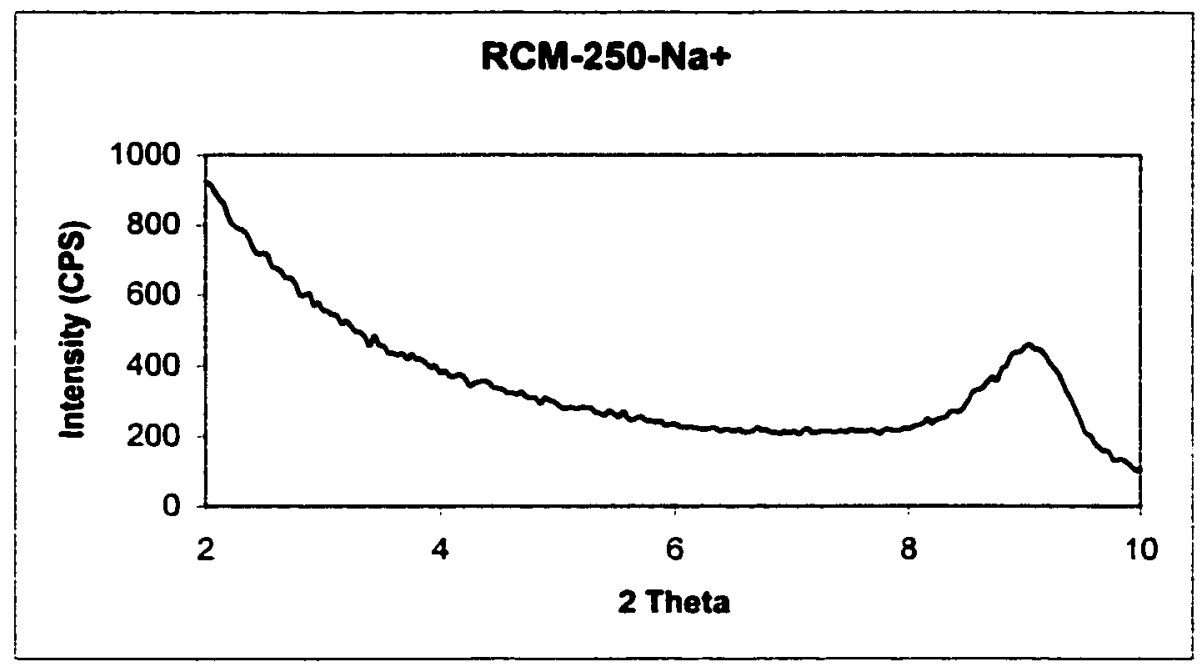

Figure 35. XRD pattern of RCM-250-Na+ clay 
This may be the result of only a single layer of alternating sodium ions instead of the double layer that is assumed to be present in the fully charged clays.

An XRD pattern of the RCM-250-Na+ dispersed in ODA-BTDA polyimide system is displayed below in Figure 36. The results show a sizeable increase in d-spacing relative to the RCM-250-Na+ by itself. This may be the result of increased interaction in the clay interior as space was opened on the clay surface for polymer interaction. These data are supported by the visual appearance of 3 given to the RCM-250-Na+film compared to the 4 for the FCM-Na+ film.

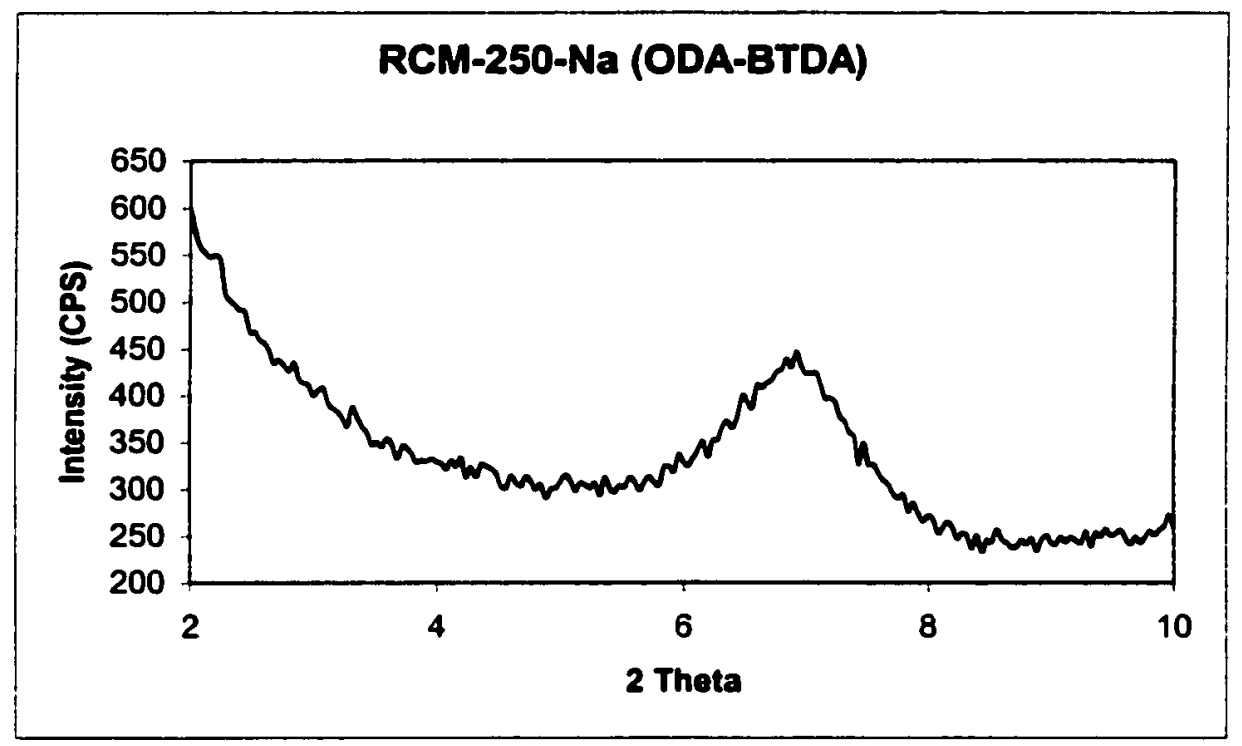

Figure 36. XRD pattern of RCM-250-Na+ ODA-BTDA hybrid film

It appeared that the charge reduction in these sodium modified clays aided in dispersing the clays in the polyimides.

3.21. Mechanical properties of APB-BPDA polyimide/organoclay hybrid films

Figure 37 compares the break stresses of hybrid films prepared using different organoclays. The films were all prepared with a $3 \%$ clay loading and the hybrids were all 
prepared via the in-situ polymerization technique. The strength values for the polyimide films that contained clay were all lower than in the neat polymer. This decrease in break stress is most likely due to the undispersed or agglomerated clay in the polymer. An important thing that was noticed concerning these data was that the strength values for the reduced charge organoclays had a slightly higher break stress than their fully charged counterpart. This could be indicative of a better dispersion when the reduced charged clays were used. The film prepared with 3\% RCM-250-BP organoclay had the highest strength for this polyimide.

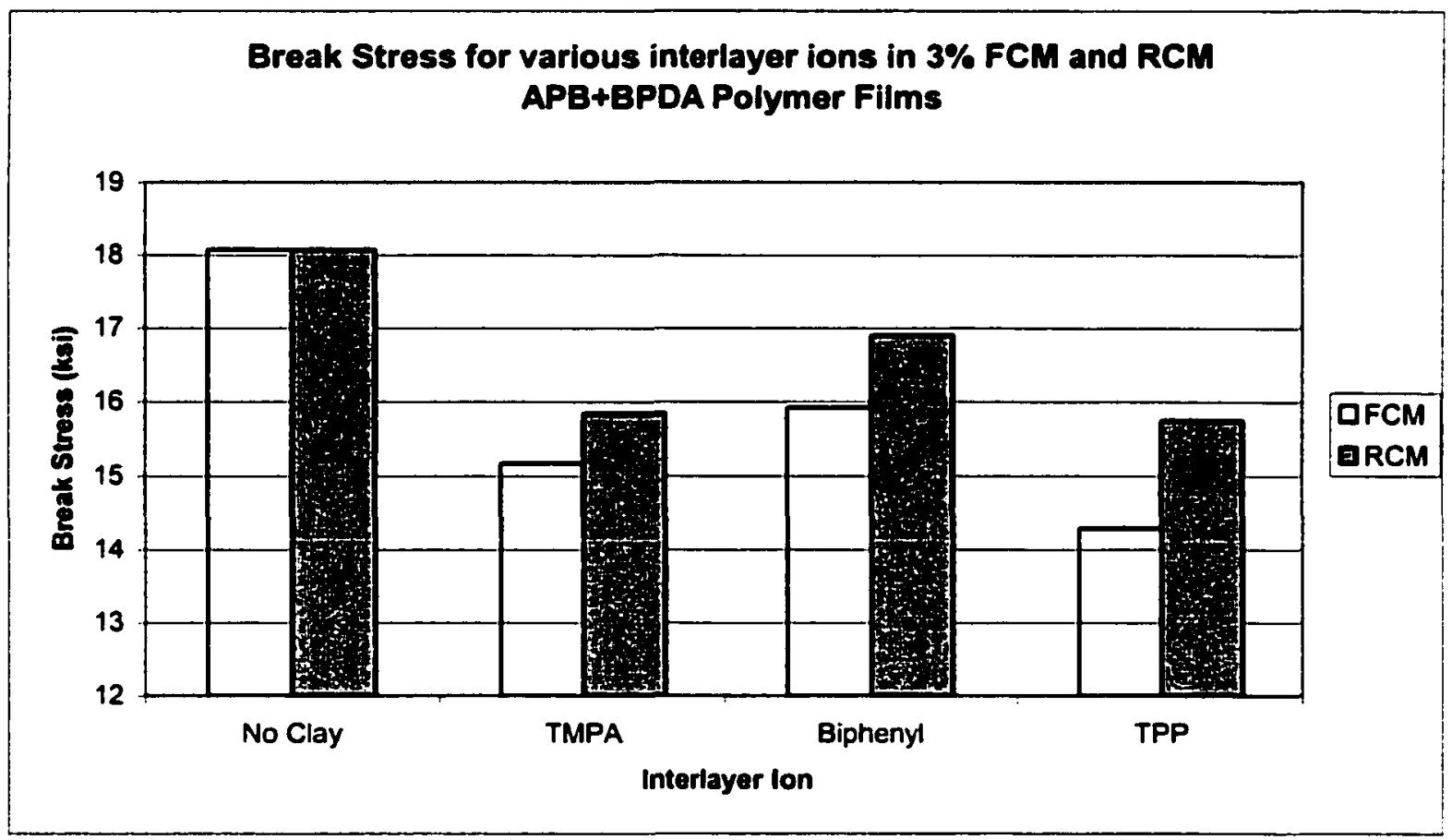

Figure 37. Strength of APB-BPDA hybrid films

Figure 38 compares modulus values for the APB-BPDA hybrids prepared with the same high temperature clays. The moduli of the FCM-TMPA and FCM-BP film are both less 
than the modulus of the neat polyimide. This is an unusual result for polymer materials filled with higher modulus particles. Most of the films prepared with the reduced charge clays had higher moduli than the FCM organoclay prepared with the same ion. The exception was the FCM-TPP film. Other characterization of these systems indicated that the FCM-TPP was slightly more dispersed than the RCM-TPP organoclay. The modulus data suggest that the RCM clays may be better dispersed than the FCM clays.

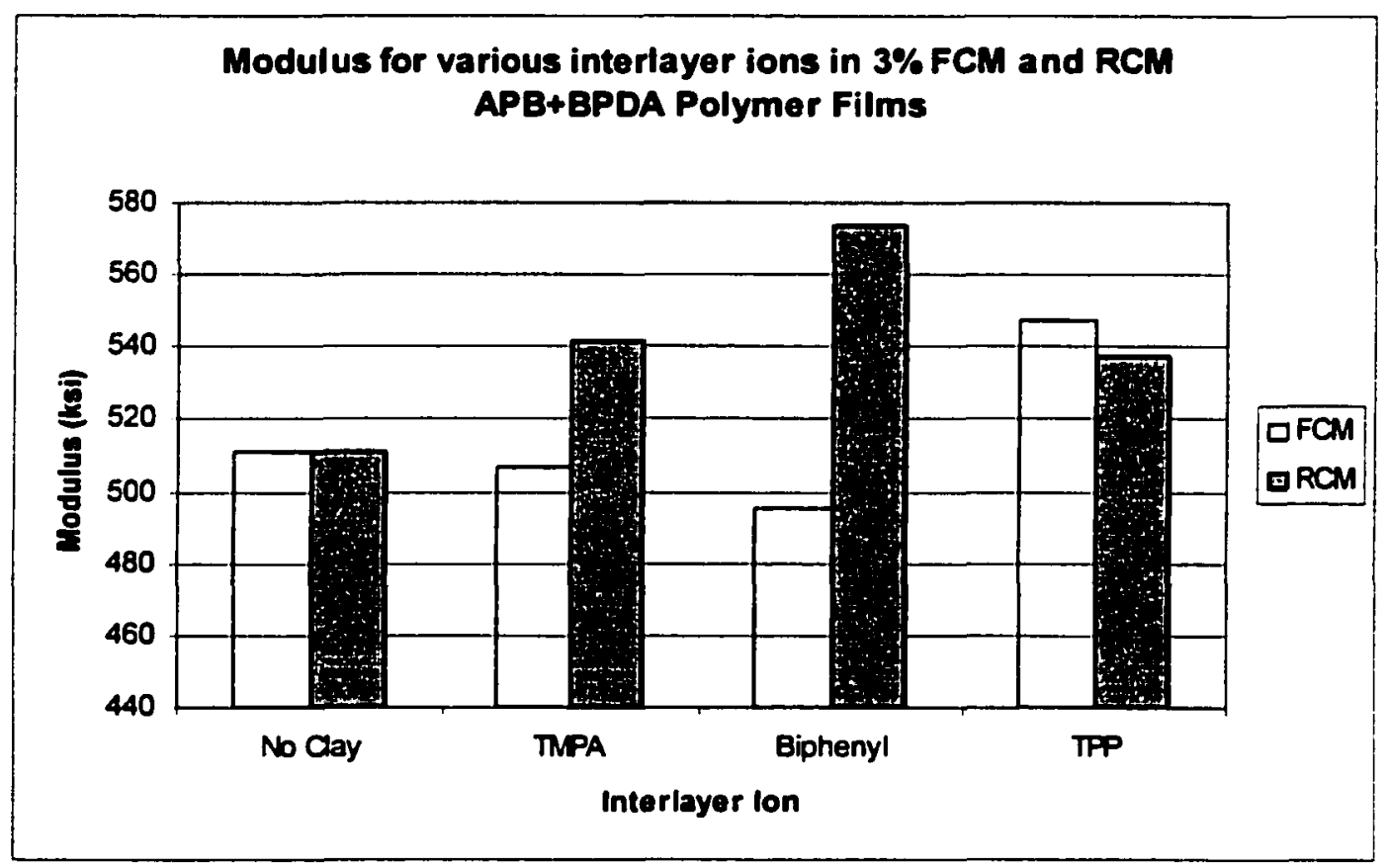

Figure 38. Modulus of APB-BPDA hybrid films 
3.22. Mechanical properties of ODA-BTDA polyimide/organoclay hybrid films

The mechanical properties of ODA-BTDA films prepared with the aromatic organoclays were measured and are reported in the figures below.

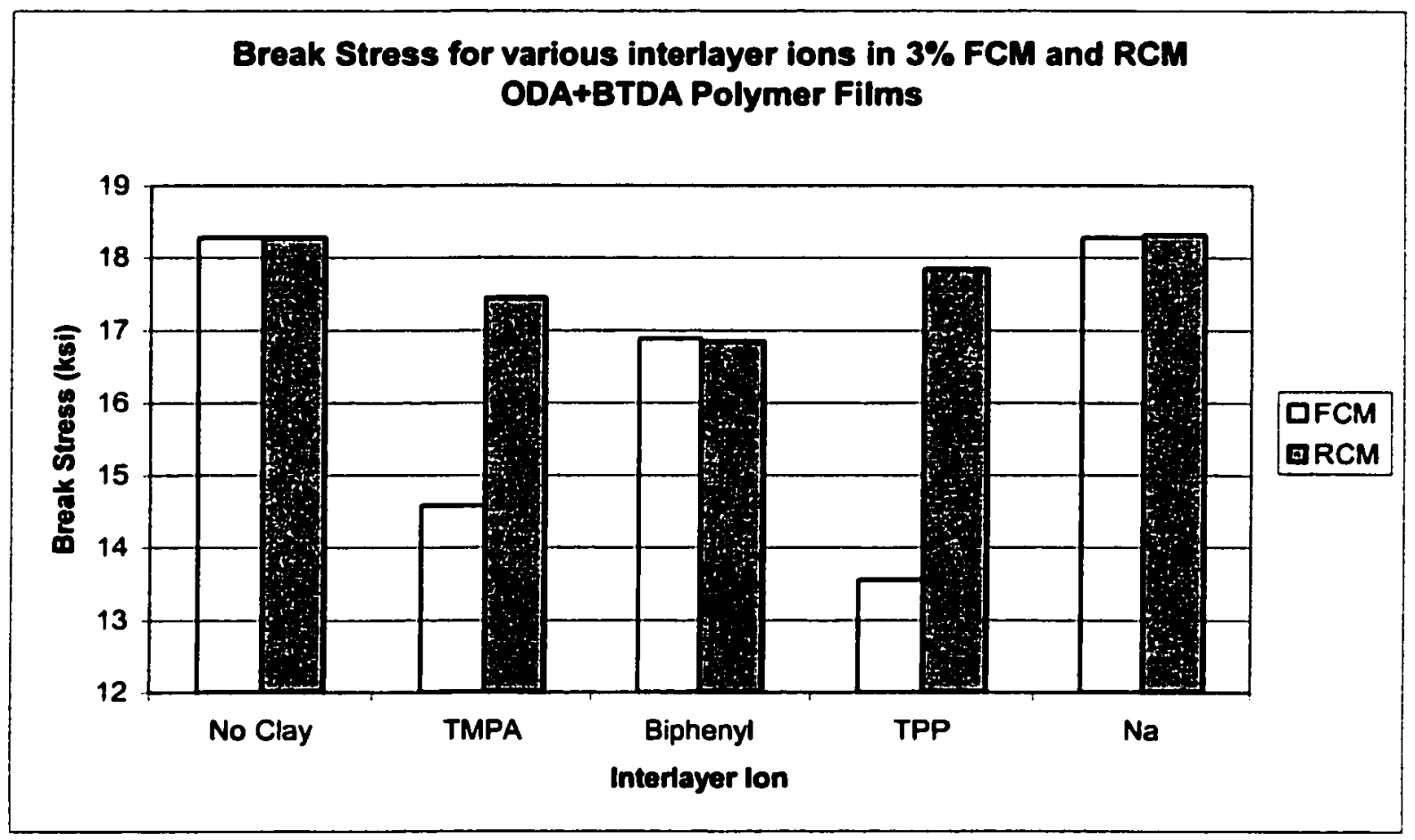

Figure 39. Strength of ODA-BTDA hybrid films

The strengths of the ODA-BTDA films prepared with various organoclays are shown in Figure 39. Within experimental error, the reduced charge clays were found to have equal or greater strengths than their FCM counterparts. All of the clay/polymer preparations have lower break stresses than the neat polymer except for the sodium clay/ODA-BTDA preparations. These films are unique because their break stress values are almost identical to the neat polymer. This was not expected because there were agglomerates in sodium clay films. 
In Figure 40 the moduli of these ODA-BTDA films prepared with various high temperature organoclays are compared. No large differences can be seen in these data that would indicate that RCM clays behaved any better than the FCM clays in these films. The RCM-250-TPP film did show the largest increase in modulus and was higher than the FCM-TPP loaded film but no conclusions could be made concerning hybrid preparations in regard to clay layer charge.

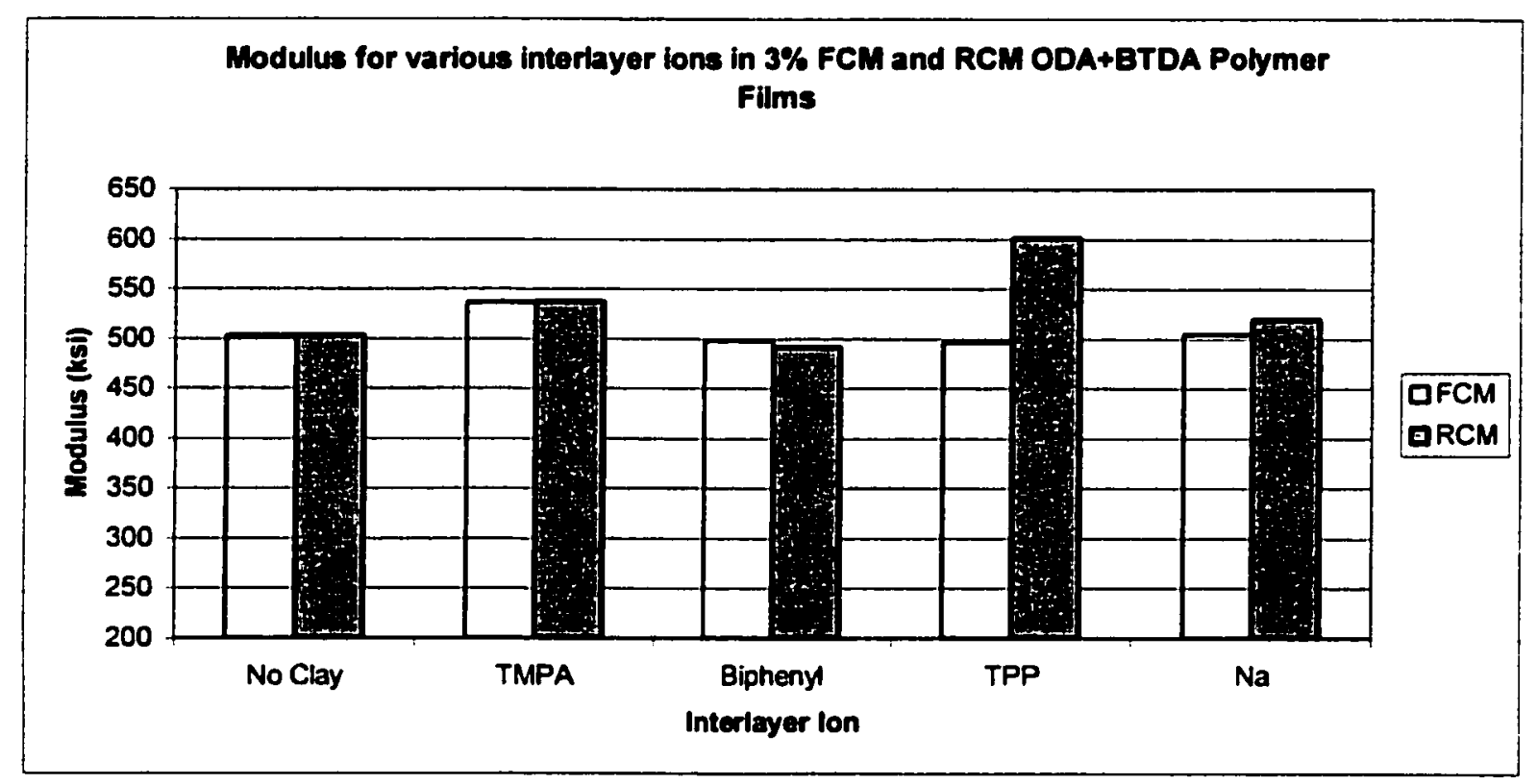

Figure 40. Modulus of ODA-BTDA hybrid films

\subsection{Thermal characterization of organoclay materials}

The purpose of this research is to design a high-temperature organoclay. This organoclay needs to have the capacity to exfoliate in polyimide matrices and also withstand the $300^{\circ} \mathrm{C}$ cure temperature of the polyimide. The dispersion characteristics are discussed in earlier sections. In order to determine if the surfactant would degrade at 
higher temperatures, the organoclays were analyzed using TGA. The temperatures at which the material exhibited 5\% weight loss are listed below.

In Figure 41 the $5 \%$ loss temperature is reported for various organoclays. In almost all of the experiments the organoclays were more stable in nitrogen atmosphere. The exception was the TPP organoclays, which were slightly less stable in nitrogen than in air. The 5\% loss temperatures of organoclays prepared with TMPA, BP, and TPP all exceeded the required $300^{\circ} \mathrm{C}$. This remained true for both the FCM and the RCM clays. These organoclays were more thermally stable than the SCPX-2003 organoclay used in Chapter III (See Figure 5, Chapter III). Other clays that are reported as high temperature such as the BK3-166C and BK3-166D (See Chapter III) both failed right at $300{ }^{\circ} \mathrm{C}$ indicating that they would not be useful clays for polyimide preparation.

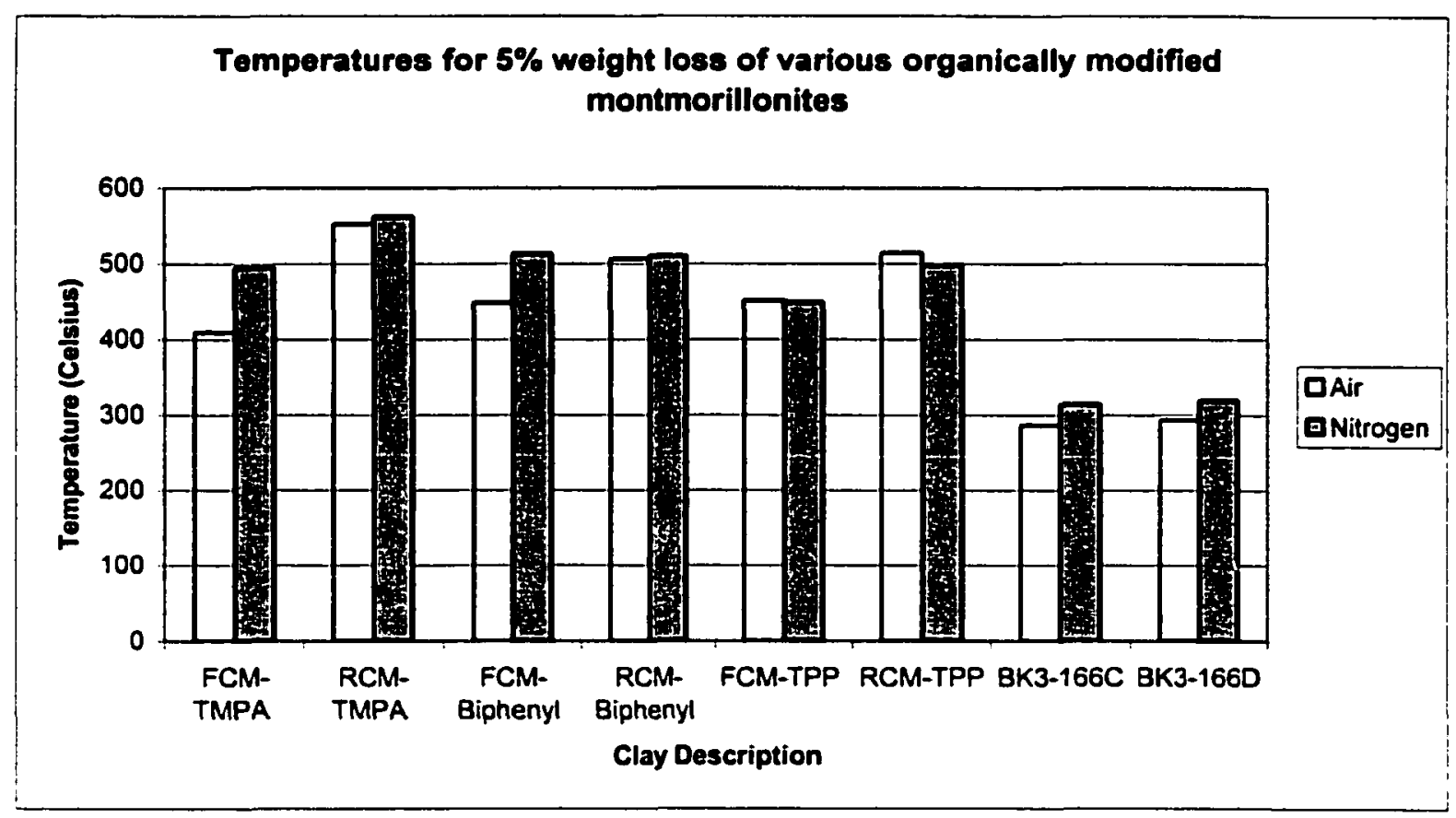

Figure 41. TGA analysis of organoclays 


\section{Conclusion}

The purpose of the work in this chapter was to provide insight into how aromatic organoclays behave in hybrid preparations. For this reason the same mixing technique as well as the same polyimides were chosen that were used in Chapter III. The high temperature organoclays that were chosen for this study were not necessarily the most attractive for use in hybrid preparation but were chosen to give some fundamental understanding.

The correlation between the d-spacing of the organoclay and its surfactant ion gave some indication as to the behavior of the surfactant ions on the surface of the clay. All the organoclays prepared with aromatic surfactant had smaller d-spacings than the aliphatic clays used in Chapter II. The reason that the aromatic organoclays did not have large gallery heights may have been an unwillingness of these ions to separate from the surface. The smaller d-spacings initially created some concern because it was thought that this would lower the driving force for the diamine monomer to enter into the gallery to begin polymer formation in the gallery. Although the lowered d-spacing was thought to be a problem the work continued with the aromatic organoclays.

Organoclays were prepared from various ions and Cloisite-Na+. The organoclays were sometimes used in the preparation of hybrid films with limited success at actually separating the organoclays into exfoliated nanoscopic particles. All of the films prepared with these organoclays had some agglomerated clay. The clay agglomerates are detrimental to the mechanical properties of the systems and are not desired. The visual analysis data for most of the films indicated poor dispersion. The film that showed the most promise was the FCM-APB/APB-BPDA which received a visual rating of 2 . Visual 
analysis of that film indicated that the organoclay was well dispersed. The visual analysis data of all the films that were prepared are presented below in Table 4.

\begin{tabular}{|l|l|l|l|}
\hline \multicolumn{1}{|c|}{ APB-BPDA } & & \multicolumn{1}{|c|}{ ODA-BTDA } & \\
\hline FCM-Na & & FCM-Na & 4 \\
\hline RCM-250-Na & & RCM-250-Na & 3 \\
\hline FCM-TMPA & 5 & FCM-TMPA & 4 \\
\hline RCM-250-TMPA & 3 & RCM-250-TMPA & 4 \\
\hline FCM-BP & 4 & FCM-BP & 4 \\
\hline RCM-250-BP & 4 & RCM-250-BP & 4 \\
\hline FCM-TPP & 3 & FCM-TPP & 4 \\
\hline RCM-250-TPP & 3 & RCM-250-TPP & 4 \\
\hline MCM-120-TMPA & 3 & & \\
\hline MCM-130-TMPA & 3 & & \\
\hline FCM-APB & 2 & & \\
\hline RCM-250-APB & 4 & & \\
\hline Table 4. Visual ana ys Of & & & \\
\hline
\end{tabular}

Table 4. Visual analysis of polyimide films

The improved visual rating in the FCM-APB/APB-BPDA film offered some evidence that the APB surfactant was more effective at modifying the clay surfaces than the other surfactants. This data was instrumental in choosing the surfactants for the final chapter of this dissertation.

The charge on the clay surface was altered to see if the clay would behave better in both the organic solvent and in the polymer matrices. For this purpose, clay was treated with lithium and held at $250^{\circ} \mathrm{C}$ for $24 \mathrm{hrs}$. This significantly reduced the charge on the 
surface of the clay but did not make a significant improvement in the hybrid material. The RCM-250-Na+/ODA-BTDA film showed some improvement over the FCM$\mathrm{Na} /$ ODA-BTDA in visual appearance but most of the hybrid preparations were similar regardless of clay surface charge.

Although the organoclays prepared with the charge reduction did not improve the hybrid materials the charge alteration was not completely abandoned. It was reported that clay could be well dispersed in poly(ethylene oxide) (PEO) nanocomposites when the clay had an intermediate surface charge ${ }^{32}$. To examine the potential of using this type of organoclay in polyimide/organoclay preparation, two intermediate clay samples were prepared and tested. Both MCM-120-TMPA and MCM-130-TMPA were placed in APB-BPDA and characterized. The results suggested that there was an improvement in the dispersion when medium charged organoclays were used. This led to studies described in the next chapter. 


\section{Chapter IV References}

1) T. Lan, P. D. Kaviratna, and T. J. Pinnavaia. Chem. Mater. 1994;6:573.

2) T. Lan and T. J. Pinnavaia. Chem. Mater. 1994;6:216;

3) M. S. Wang and T. J. Pinnavaia. Chem. Mater. 1994;6:648;

4) T. Lan, P. D. Kaviratna, and T. J. Pinnavaia. Chem. Mater.1995;7: 2144.

5) T. J. Pinnavaia, T. Lan, P. D. Kaviratna, and M. Wang, Clay-Polymer Nanocomposites: Polyether and Polyimide Systems, Mater. Res. Soc. Symp., Paper N2.8 (1994);

6) P. D. Kaviratna, T. Lan, and T. J. Pinnavaia. Polym. Prepr. (Am. Chem. Soc., Polym. Div.) 1994;35:788.

7) E. R. Kleinfeld and G. S. Ferguson. Science 1994;265:370;

8) E. R. Kleinfeld and G. S. Ferguson. Chem. Mater 1995;7:2327.

9) P. B. Messersmith and E. P Giannellis. Chem. Mater. 1994;6:1719.

10) S. H. Burnside and E. P. Giannellis. Chem. Mater. 1995;7:1597.

11) P. B. Messersmith and E. P Giannellis. J. Polym. Sci A. 1996;33:1047.

12) Y. Fukushima, S. Inagaki. J. Inclusion Phenom. 1987;5:473.

13) Y. Fukushima, A. Okada, M. Kawasumi, T. Kurauchi, and O. Kamigaito. Clay Miner. 1988; $23: 27$.

14) A. Usuki, M. Kawasumi, Y. Kojima, A. Okada, T. Kurauchi, and O. Kamigaito. J. Mater. Res. 1993;8:1174.

15) A. Usuki, Y. Kojima, M. Kawasumi, A. Okada, Y. Fukushima, T. Kurauchi, and O. Kamigaito. J. Mater. Res. 1993;8:1179. 
16) Y. Kojima, A. Usuki, M. Kawasumi, A. Okada, Y. Fukushima, T. Kurauchi, and O. Kamigaito. J. Mater. Res. 1993;8:1185.

17) K. Yano, A. Usuki, A. Okada, T. Kurauchi, and O. Kamigaito. J. Polym. Sci., Polym. Chem. Ed. 1993;31:2493.

18) K. Yano, A. Usuki, A. Okada, T. Kurauchi, and O. Kamigaito. Polym. Prepr. (Am. Chem. Soc., Polym. Div.) 1991;32:65.

19) P. Lebaron, Z. Wang, T. Pinnavaia. Applied Clay Science 1999;15:11.

20) G. Lagaly. Applied Clay Science 1999;15:1.

21) D. Delozier, R. Orwoll, J. Cahoon, N. Johnston, J. Smith, and J. Connell. Polymer 2002;43:813.

22) Y. Yang, Z. Zhu, J. Yin, X. Wang, and Z. Qi. Polymer 1999;40:4407.

23) H. Tyan, K.Wei, T. Hsieh, J. Poly. Sci., Part B, Poly, Phys. 2000;38:2873.

24) H. Tyan, K. Wei, T. Hsieh. Chem. Mater. 1999;11:1942.

25) Tyan et.al. Chem. Mat. 2001;13:222.

26) W. Zhou, J. Mark, M. Unroe, and F. Amold, J. Macromoleecular Sci.-Pure Appl. Chem. A38(1), 1-9 (2001).

27) R. Vaia, E. Gianellis. Macromolecules 1997;30:8000.

28) B. Theng. The Chemistry of Clay-Organic Reactions, New York, 1974.

29) J. Stevens and S.Anderson. Clays and Clay Minerals 1996;44:132.

30) J. Budjak and P. Komadel, J. Phys. Chem. B 1997;101:9065.

31) W. Jaynes and J. M. Bigham. Clays and Clay Minerals 1987;33:440.

32) J. Budjak, E. Hackett, and E. Gianellis. Chem. Mater. 2000:2168.

33) Vaia, Teukolsky, \& Giannelis, Chem. Mater 1994;6:1017 and references therein. 


\section{Chapter V}

In-situ polymerization with charge optimized, high temperature organoclays

\section{Introduction}

Unique problems were encountered when trying to prepare nanocomposite materials in which polyimides serve as the matrix for clay platelets. The chemical structure of the polyimide backbone and the high temperatures at which the polymer needs to be heated to cure the polyimide were the cause of the problems. The polyimides are usually highly aromatic along the polymer backbone and require heating to $300^{\circ} \mathrm{C}$ to effect imidization. Due to the nature of the polyimides they would not simply mix with most organoclays. The in-situ polymerization technique was a remedy to the mixing problem when aliphatic organoclays were used. Although the in-situ method was effective at dispersing these organoclays to nanoscopic dimensions, the low thermal stability of the aliphatic quaternary amines proved to be a problem in preparing polyimide nanocomposites ${ }^{1,2}$. Preparing nanocomposite materials using in-situ polymerization with high-temperature organoclays was discussed in Chapter IV. The high-temperature, aromatic organoclays were more difficult to disperse than the SCPX2003 organoclay and other aliphatic organoclays. Although they were more difficult to disperse there was some indication that altering the charge aided in dispersion and the medium charged clays appeared to be the most effective. The use of APB as the surfactant also offered some promising results in the previous section. Although the task of dispersing aromatic organoclays in the polyimides proved challenging, the information 
obtained in the earlier chapters gave indication that the dispersion of aromatic organoclays was possible.

Aromatic organoclays have been studied for a variety of purposes. The use of aromatic amines as ion exchange compounds to render clay surfaces more organophilic was first used outside the polymer industry in the pesticide ${ }^{3}$ and water purification industries ${ }^{4}$. Aromatic ions were also effective at making clays effective sorbent materials ${ }^{5}$. Other studies involving aromatic ions were aimed at preparing organoclays for nanocomposite preparation. There are a few reported attempts at preparing organoclays for use in polyimide nanocomposite preparation. One attempt was the use of aromatic surfactant ions that mimicked the diamine monomer. The dihydrochloride salt of 4,4' oxydianiline (ODA) was used in the preparation of ODA-4,4'-benzophenone tetracarboxylic dianhydride (BTDA) nanocomposites by Tyan and coworkers ${ }^{6}$. A trifunctional amine salt that had high temperature capabilities was also used in preparing polyimide nanocomposites ${ }^{7}$. The use of aromatic ions in organoclay preparation and subsequent nanocomposite preparation has been reported to be successful in some instances ${ }^{8}$. Although successful reports of exfoliating clay particles using aromatic surfactants in polyimide nanocomposite preparation are available, the repeatability and reproducibility has proven difficult. The research presented herein describes further alterations to the clay surface that make aromatic organoclays more effective in preparing polyimide nanocomposites.

The fundamental interaction that governs the behavior of organoclays in polymer matrices is the interaction at the polymer/clay interface. The surfaces of organoclays are treated with an organic surfactant which becomes a critical part of this interface. A paper 
by Vaia and Gianellis, which modeled the polymer-surfactant-clay interaction, was concluded by stating that an effective way to increase the polymer/clay surface interaction was to increase the available clay surface while decreasing the negative interactions between polymer and surfactant ${ }^{9}$. The negative interactions are those repulsive forces between the polymer backbone and the surfactant caused by structural differences in the two. The attempts at making these interactions more favorable in our work involved decreasing the layer charge of the montmorillonite prior to ion exchange and using aromatic moieties as the surfactant. This reduction in layer charge has shown to increase the affinity of PEO for montmorillonite clays ${ }^{10}$ as well as methylene blue for clay surfaces ${ }^{11}$. The method of charge reduction is clearly defined in the previous literature ${ }^{12}$ and is accomplished by lithium migration into the interlayer.

The work presented in this chapter was focused on designing the most favorable polymer/clay interaction with the APB-BPDA polyimide and two different organoclays. The majority of the work was focused on determining an optimum surface layer charge for the exfoliation of montmorillonite clays in this polyimide system. The effects of layer charge and surfactant were measured by characterizing the physical and mechanical properties of the polyimide nanocomposite films. 


\section{Experimental}

\subsection{Starting materials}

The organically modified clay (SCPX-2003) and Cloisite $(\mathrm{Na}+)$ montmorrillonite were used for the clay materials in this work. APB and BPDA were used to prepare the polymer materials.

High purity, anhydrous N-methyl-2-pyrrolidinone (NMP), trimethyl phenyl ammonium (TMPA), 3-aetamidophenol, potassium hydroxide $(\mathrm{KOH})$, potassium iodide (KI), and 1,5-dichloro-3-oxapentane were purchased from Aldrich and used as received.

\subsection{Preparation of poly(amide acid)s \\ Refer to Section 2.6 in Chapter II.}

2.3. Preparation of poly(amide acid)/organoclay hybrids via in-situ polymerization Refer to Section 2.5 in Chapter III.

\subsection{Preparation of dihydrochloride salts}

The following is a representative procedure for preparing 1,5 bis(3aminophenoxy-3-oxapentane dihydrochloride (BAOD) ${ }^{13}$.

Into a $250-\mathrm{mL}$ three-necked round bottom flask equipped with a stopper, mechanical stirrer, and a condenser was placed 3-acetamidophenol $(40 \mathrm{~g}) .100 \mathrm{~mL}$ of 
ethanol was added to the flask and the reaction was stirred. $\mathrm{KOH}(14.87 \mathrm{~g}), \mathrm{KI}(39.84 \mathrm{~g})$ and water $(50 \mathrm{~mL})$ were then introduced. The mixture was heated to reflux while stirring. The mixture was light yellow prior to heating. On heating, the reactants dissolved yielding a clear yellow solution. 1,5-Dichloro-3-oxapentane (17.28 $\mathrm{g})$ was added to the solution in five equal amounts over five hours. After being refluxed for 48 hours, the solution was slowly cooled before being placed in a freezer at $-2^{\circ} \mathrm{C}$ for $24 \mathrm{hrs}$. The white precipitate that formed in the flask was collected in a Büchner funnel and washed repetitively with water until the filtrate was clear. The product was recrystallized in ethanol yielding a white powder (m.p. $150^{\circ} \mathrm{C}$ ) instead of large crystals. The powder (18.6g) was introduced to a $1000-\mathrm{mL}$ round-bottom flask with concentrated $\mathrm{HCl}(90.73$ $\mathrm{mL})$, ethanol $(181.46 \mathrm{~mL})$, and water $(45.37 \mathrm{~mL})$. The mixture was heated to reflux for 4 hours yielding a clear brown solution. The mixture was slowly cooled to room temperature yielding a yellow precipitate. The solid was collected in a Büchner funnel and was washed successively with ethyl acetate, acetone, and cold $95 \%$ ethanol. The product (m.p. $230^{\circ} \mathrm{C}$, lit. m.p. $230^{\circ} \mathrm{C}^{13}$ ) was dried under vacuum.

Refer to Section 2.3 in Chapter IV for a representative procedure for preparing the dihydrochloride salt of APB.

\subsection{Preparation of reduced charge clays}

Into a 500-mL beaker was placed Cloisite $\mathrm{Na}+(15 \mathrm{~g})$ and $1000 \mathrm{~mL}$ of aqueous $1 \mathrm{~N}$ lithium chloride. The clay suspension was homogenized using a Powergen ${ }^{\mathrm{TM}}$ homogenizer for 5 minutes until a homogenous suspension was reached. The suspension 
was subsequently transferred to polypropylene centrifuge tubes and centrifuged at 13,000 rpm for 10 minutes yielding clay pellets. The clay was placed in $1000 \mathrm{~mL}$ lithium chloride solution and again the suspension was homogenized. This was repeated once more making 3 consecutive washings in $1 \mathrm{~N} \mathrm{LiCl}$. The clay was centrifuged after the last $\mathrm{LiCl}$ washing and then suspended in deionized water via homogenization. This washing in water was repeated three times. The clay was removed from the centrifuge tubes and placed in separate platinum crucibles in a flowing air oven. The crucibles were heated for $24 \mathrm{hrs}$. One sample was heated to $120^{\circ} \mathrm{C}$; others were heated to $130,140,150,160$, and $170{ }^{\circ} \mathrm{C}$. After cooling to ambient temperatures the samples were ground to a fine powder in a ball mill.

\subsection{Preparation of organoclays}

The following is a representative procedure for preparing organically modified montmorrillonite clays.

Into a 250-mL fleaker was placed Cloisite $\mathrm{Na}+(1.0026 \mathrm{~g})$ and $100 \mathrm{~mL}$ of deionized water. The clay was homogenized until a stable suspension was reached $(\sim 5$ minutes). In a separate $25-\mathrm{mL}$ beaker was placed the dihydrochloride salt of APB (0.6 $\mathrm{g})$ and $20 \mathrm{~mL}$ of deionized water. The salt completely dissolved with stirring using a magnetic stir bar. The resulting solution was added dropwise to the clay suspension while sonicating. Sonication was continued for approximately $\mathrm{l}$ hr to ensure good mixing. This mixture was then introduced to a Soxhlet thimble and extracted for $24 \mathrm{hrs}$ with deionized water. The clay was lyophilized and stored in a dessicator prior to use. 
2.7. Unoriented thin films

Refer to Section 2.7 in Chapter II.

\subsection{Determination of Layer Charge}

Powdered, heat-treated lithium clay $(0.2 \mathrm{~g})$ as described in Section 2.5 above and water $(20 \mathrm{~mL})$ were added to a $50-\mathrm{mL}$ polypropylene centrifuge tube and sonicated until a homogenous clay/water suspension was reached in the tube. A separate aqueous solution of ammonium acetate was added dropwise to the clay suspension. This mixture was sonicated for 10 minutes. The clay was compacted into a pellet by centrifugation. The clear aqueous supernatant was analyzed for lithium with atomic absorption. The clay was then resuspended in $20 \mathrm{~mL}$ of deionized water with sonication, treated with ammonium acetate in a sonicator for 10 minutes and then centrifuged to a pellet. The supernatant was again analyzed for lithium. This cycle was repeated one more time. The total lithium that was removed from the clay was calculated.

A series of control samples was prepared using the same powdered clays.

However, these clays were washed with deionized water without ammonium acetate. The lithium concentration in the extract was measured. The difference between the quantity of lithium ions found in the extract after ammonium acetate washing and that measured from the washings with pure water was considered the exchangeable lithium.

The exchangeable lithium content was measured on FCM-Li, MCM-120-Li, MCM-130-Li, MCM-140-Li, and MCM-150-Li clays and the results are reported*.

* Abbreviations are described below 


\subsection{Description of clay abbreviations}

The description given to the clay combinations used in the research presented herein is described in Table 1.

\begin{tabular}{|l|l|}
\hline Abbreviation & Description \\
\hline FCM-APB & Fully charged Cloisite-Na+ ion exchanged with APB+ \\
\hline FCM-BAOD & Fully charged Cloisite-Na+ ion exchanged with BAOD \\
\hline MCM-120-APB & $\begin{array}{l}\text { Lithium treated montmorillonite clay that was held at } \\
120^{\circ} \mathrm{C} \text { for } 24 \mathrm{hrs} \text { and ion exchanged with APB+ }\end{array}$ \\
\hline MCM-120-BAOD & $\begin{array}{l}\text { Lithium treated montmorillonite clay that was held at } \\
120^{\circ} \mathrm{C} \text { for } 24 \mathrm{hrs} \text { and ion exchanged with BAOD }\end{array}$ \\
\hline
\end{tabular}

Table 1. Description of abbreviations

All the clays that are mentioned in the text have the same general formula for abbreviation. The type of clay is mentioned first, the heating temperature is next, and the ion that is in the inner layer is mentioned last.

\subsection{Other Characterization}

Atomic absorption data were collected on a Perkin Elmer 1100B Atomic Absorption Spectrophotometer. For all other characterization refer to Section 2.7 in Chapter III.

\section{Results and Discussion}

3.1. Preparation of organoclays via ion-exchange

Two aromatic surfactant molecules were chosen for this study and were used to prepare organoclays. The polyimide that was chosen for this research was APB-BPDA 
and the dihydrochloride salt of the diamine (APB) was used in the preparation of one of the organoclays. Another organoclay was prepared with a surfactant (BAOD) with aromatic endgroups that also had a non-aromatic ethylene oxide interior region. The meta ether linked APB chain has some flexibility due to the meta linkages and is the same as the monomer used in the preparation of the PAA. This molecule was predicted to behave well as a surfactant molecule for these reasons. The BAOD salt should be very flexible due to the ethylene oxide interior region and it possesses the necessary thermal properties for use in the organoclays. The two structures are shown below in Figure 1.<smiles>Nc1cccc(OCCCOc2cccc([N+](=O)[O-])c2)c1</smiles>

BAOD<smiles>Nc1cccc(Oc2cccc(Oc3cccc(N)c3)c2)c1</smiles>

APB

Figure 1. Structure of surfactant ions

The preparation of organoclays involves an ion exchange reaction in which an organic ion is exchanged for an inorganic ion. Both of the surfactants are water-soluble ions, thus the exchange reactions were performed in water. In the preparation of organoclays the sodium or lithium clay was suspended in water. The clay suspension was effectively converted into a sol via sonication. The sol was composed of a $1 \%$ clay suspension in water. The clay remained suspended for hours in the absence of excess salt. A $5 \%$ by weight salt solution in water was prepared and was added dropwise to the clay sol while sonicating. The total amount of the salt that was added for all the clays regardless of charge was approximately a two-fold excess of the predicted CEC of the fully charged montmorillonite. In previous chapters other methods of stirring, such as homogenizing and stirring with a magnetic stir bar, were also attempted for mixing 
purposes when the salt was being added. These other methods were also effective but sonication was chosen as the primary means of mixing the two components. The organoclays were allowed to sonicate for $\sim \mathrm{hr}$ to ensure good mixing and the final water temperature was $\sim 45^{\circ} \mathrm{C}$.

After adding the salt solution during the preparation of the organoclays, the clay particles flocculated. In the more reduced charge clays (MCM-140, MCM-160, and MCM-170) the flocculation resulted in small pellets that did not stay suspended. These clays started turning orange almost immediately after introduction of the APB + salt and turned light blue after BAOD introduction. The respective colors became darker after sonicating for the full hour. The FCM, MCM-120, and MCM-130 clays flocculated into a very voluminous mass after salt introduction. This mass floated in the water for a while if not agitated. During ion exchange these clays would start as a non-colored mass and the APB clay would slowly become darker orange while the BAOD would become darker blue while sonicating.

The clays were washed free of excess salt by Soxhlet extraction. The clays were placed in a cellulose thimble and deionized water was flushed over the system. The clays became darker as they were left in the extractor for longer times. It was later confirmed that the increased temperature caused the color gain in all the clays that were extracted. Excess heat (above $70^{\circ} \mathrm{C}$ ) was detrimental to the swelling properties of the organoclays. To determine the best charge for organoclay preparation the organoclays were screened for performance in nanocomposite preparation. This initial screening experiment was performed with clays that were extracted for $\sim 8 \mathrm{hrs}$. After the clays were extracted they were all lyopholized yielding a fluffy, powdery material. The APB + organoclays were 
orange while the BAOD clays were light blue after freeze drying. The lower charged clays were always darker than the more highly charged clays. For example the MCM170-APB was darker orange than the FCM material prepared the same way. This same trend held true for the BAOD materials. The FCM-BAOD organoclay was only light blue while the MCM-170-BAOD organoclay was dark blue.

Changes were made to the experimental procedure in the experiment with the optimally charged clays. Longer extraction times ( $24 \mathrm{hrs)}$ were used in this nonscreening experiment. The organoclays prepared in this manner were a little darker than similar clays that were only extracted for $8 \mathrm{hrs}$. Although these organoclays were slightly more colored, they behaved better in solvent and ultimately yielded better results in nanocomposite preparation. It was also determined that the clays did not have to be ion-exchanged with $\mathrm{Na}+$ prior to organoclay preparation. Although the organoclays that were used in the screening experiment were exchanged with sodium prior to use, this step was left out of the procedure for the clays that were used in the preparation of 3,5 , and 8\% MCM-130-APB/APB-BPDA and MCM-130-BAOD/APB-BPDA films.

\subsection{Preparation of polyimide/organoclay hybrids via in-situ polymerization}

It has been established that the best preparatory technique in polyimide/organoclay preparation is in-situ polymerization ${ }^{l}$. This technique was used in the preparation of all the hybrids discussed below. In all the hybrid preparations the clay was dispersed in NMP prior to introduction to polymer. This was always accomplished by placing the clay in a volumetric flask with NMP and sonicating until the clay suspension was well 
dispersed. The homogeneity of the organoclay/NMP suspension was a good indicator of clay dispersion and clarity of the PAA mixture. The appearance of the clay suspension was directly linked to how well the clay particles were dispersed in the polyimide film. For example, when the charge had been reduced too much the clay settled to the bottom in the organoclay/NMP suspension. Nanocomposites prepared from these solutions contained poor dispersion. In contrast, the suspensions of fully charged clays the clay would remain suspended and the suspensions were clear. Although the appearance of these suspensions were improved relative to the reduced charge dispersions, if the suspension was swirled or shaken two different phases were seen in the suspension. The citimally charged (MCM-130-APB) organoclays tended to take on the flat appearance of the FCM clays but no differences in the clay suspension were seen if the suspension was shaken. The optimally charged MCM-130-APB suspensions were also very viscous. When MCM-130-APB was used, the best dispersion was achieved in the polyimide films. As stated above, clay suspensions of the MCM-130-APB organoclay were also the most homogenous in appearance, clearest, and most viscous when suspended in NMP. A somewhat different result occurred when BAOD was used as the surfactant. When BAOD was used as the surfactant the best dispersion was achieved in the MCM-130BAOD/APB-BPDA film but the FCM-BAOD organoclay seemed to have slightly better clarity when placed in NMP. Although the optical clarity was not as good in the MCM130-BAOD suspension in NMP as the FCM-BAOD suspension, the MCM-130-APB suspension was more viscous than the FCM-BAOD/NMP suspension. Although the argument is not as persuasive as in the APB organoclays, the appearance and viscosity of 
the BAOD organoclay suspensions seemed to be directly linked to the performance of the clay in hybrid formation.

The organoclay suspensions were stirred in nitrogen atmosphere prior to monomer addition. The APB+ organoclay/NMP suspensions were yellow when the clays had a higher charge and were amber when the lower charged clays were used. The fully charged BAOD clays were greenish black when dispersed in NMP. The optimally charged BAOD clay suspensions were darker while the highly reduced charge BAOD clay suspensions were black. The monomers were added to the suspensions stepwise to effectively perform in-situ polymerization. The monomers that were used are shown in Figure 2.
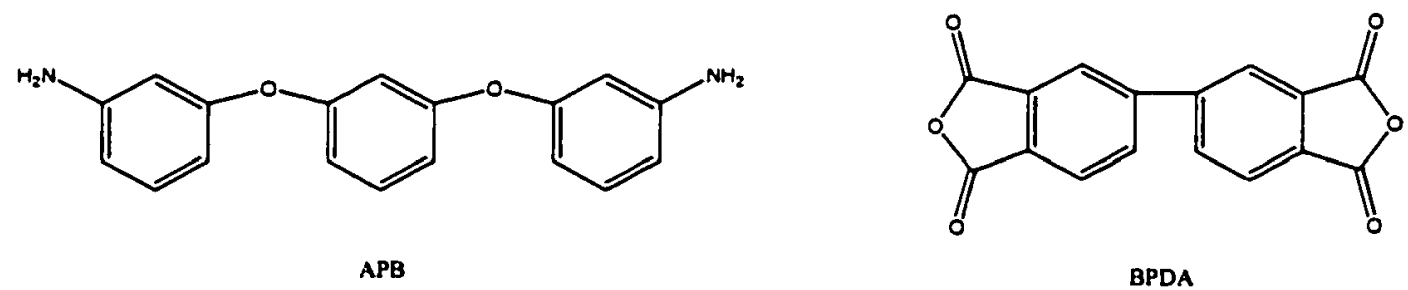

Figure 2. Structure of monomers

The diamine was allowed to dissolve in the organoclay/solvent suspension prior to the addition of the dianhydride. This presumably allowed the diamine to interact with the clay interlayer. The mixture did not change in visual appearance after the diamine was introduced in any of the systems. After the dianhydride was introduced, the polymer started to form in the interlayer and the mixture became more viscous. The APB organoclay suspensions were all yellowish to amber suspensions while the BAOD suspensions were greenish black to black after polymer chain growth.

The focus of the initial research was to screen organoclays in hopes of discovering an organoclay that would exfoliate in the chosen polyimide. The first set of 
experiments involved preparing APB-BPDA PAA in the presence of different organoclays. The organoclay charge and surfactant were altered in the different organoclays. The hybrid mixtures were used in the preparation of polyimide films. The $3 \%$ clay loaded films prepared with the FCM-BAOD and FCM-APB were amber and clear. The MCM-120-APB and MCM-120-BAOD films were also fairly clear but had begun to show slight scattering of light. This effect was even more pronounced in the films prepared with MCM-130 organoclays. There was a large amount of light scattering throughout the entire film. The films that were prepared with MCM-140 through MCM170 organoclays of both APB+ and BAOD did not have this scattering effect but started to take on a hazy appearance. The polymer films were characterized with XRD, tensile tests, and TEM. After this set of experiments was performed it was assumed that the optimally charged organoclay when either APB + or BAOD were used as the surfactants was the MCM-130 clay. After deciding that these organoclays dispersed well in APBBPDA it was of interest to further investigate the MCM-130 type systems.

The MCM-130-APB and the MCM-130-BAOD were used in a second experiment that looked at higher clay loadings in the nanocomposite materials. Slight modifications were made in the preparation of the organoclays and the clays were tried at different loading levels $(3 \%, 5 \%, 8 \%)$. The modifications that were made in organoclay preparation consisted of extending the Soxhlet extraction time to $24 \mathrm{hrs}$ and not exchanging the lithium with sodium prior to ion exchange. These organoclays were used in the hybrid preparation and yielded slightly better results in the characterization of the hybrid films. The PAA/organoclay mixtures that were prepared with these clays were a little darker and the darkening was attributed to the extended time the organoclay spent in 
the Soxhlet extractor. The preparation of the hybrid mixtures and films was kept the same when higher loading levels were used. The hybrid films all had the same light scattering effect as the MCM-130-APB/APB-BPDA and MCM-130-BAOD/APB-BPDA films used in the screening experiment with the MCM-130 clays. The scattering effect was even more pronounced at the higher loadings. The films that contained higher concentrations of clay were also more colored and were slightly more brittle. These films were characterized and the data is presented below.

\subsection{XRD analysis of $\mathrm{Na}+$ clays}

Prior to ion exchange with an organic salt the clays have a d-spacing that is smaller than their organically exchanged counterpart. The XRD pattern of Cloisite- $\mathrm{Na}+$ clay is shown in Figure 3.

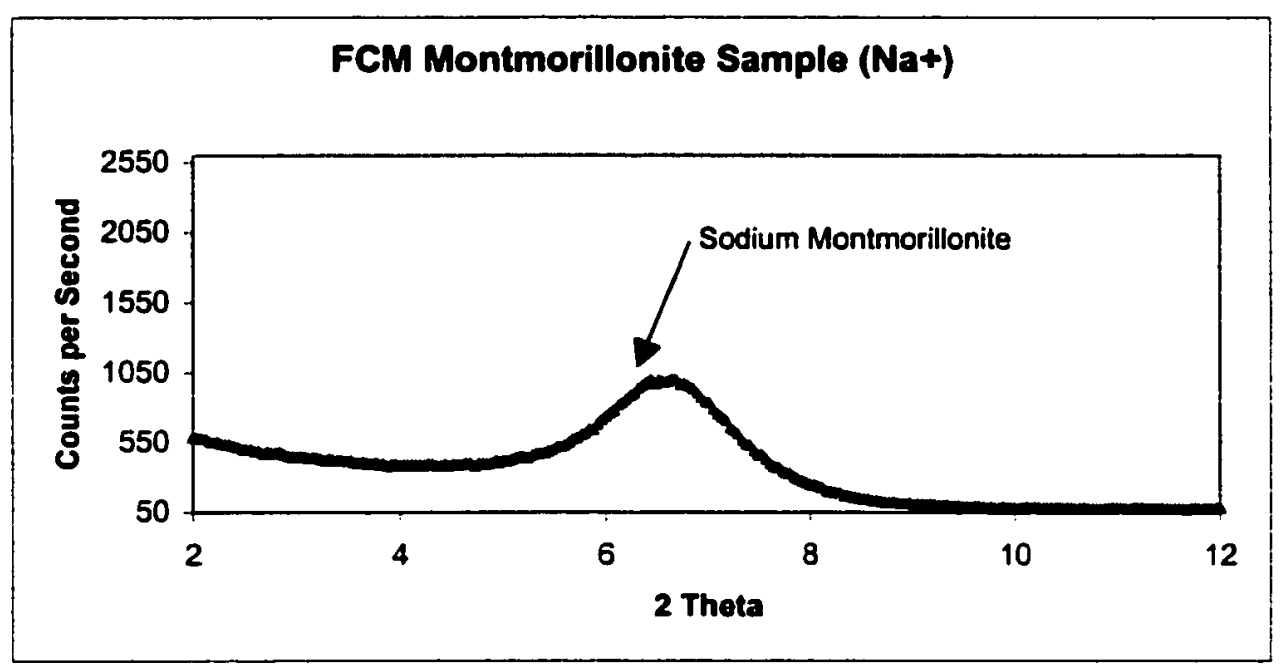

Figure 3. XRD pattern of sodium montmorillonite

The Cloisite- $\mathrm{Na}+$ had a $\mathrm{d}$-spacing of $1.3 \mathrm{~nm}$ as shown by the $\mathrm{XRD}$ peak at $2 \theta=6.6^{\circ}$. This clay was used in the preparation of the FCM organoclays. This clay was also the clay that was exchanged with lithium and heat treated to produce all of the MCM clays. The 
FCM clay had a large d-spacing relative to the MCM clays. The XRD patterns for the MCM clays that were changed back to the sodium form after heat treatment are shown in Figure 4.

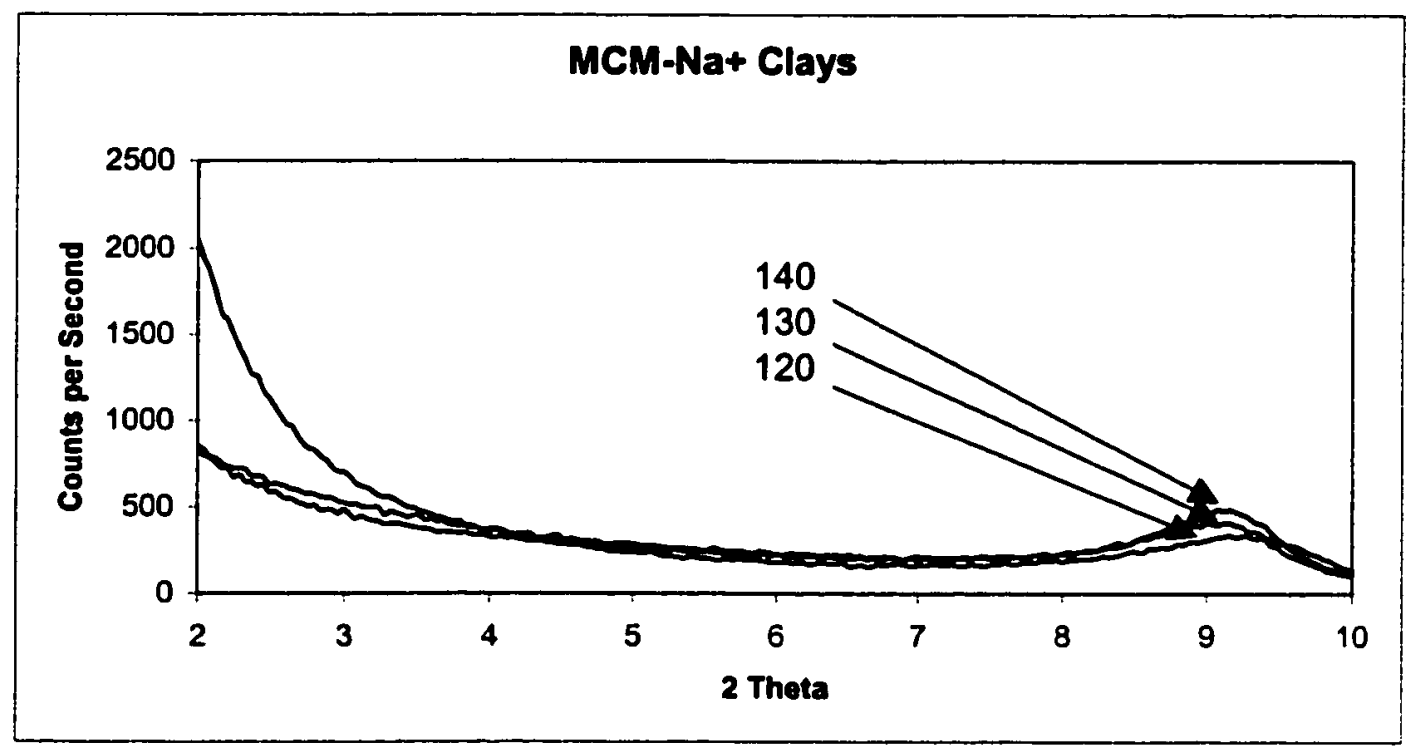

Figure 4. XRD pattern of MCM-Na+ clays

These clays were chosen as representative samples to determine what happened to the $d$ spacing of the clays after the charge was reduced. The MCM clays all had roughly the same d-spacing $(-.95 \mathrm{~nm})$ after the heat treatment. Again when compared to the fully charged sample there was a large reduction in the layer spacing due to the heat treatment. The XRD patterns for the organoclays (Figures 5-7) together with the data in Figure 4 indicated that the silicate framework stayed the same size while there was a loss of inner layer water as layer charge was reduced. This was suggested because the FCM organoclays had roughly the same d-spacing as the 120,130 , and 140 organoclays. A monolayer of water coordinated to the sodium ions was most likely the only thing present in the inner layer of the clays after lithium treatment and heating ${ }^{10}$. 


\subsection{XRD analysis of organoclays}

Organoclays were prepared via ion exchange reactions with organic amine salts. The organoclays displayed different characteristics after the ion exchange reaction including color changes and their behavior in organic solvents. The layer spacings were examined to obtain a better understanding of the aromatic salt/clay interaction. The organoclay layer spacing was examined for both the FCM and reduced charge organoclays. The more highly charged clays were expected to have a greater d-spacing as the amine salts have less space to lie flat against the surface. As the charge was reduced the trend was expected to reverse as the aromatic salts had more room to get closer to the surface. The different APB organoclay preparations were examined via $\mathrm{XRD}$ and the results are shown below in Figures 5 and 6.

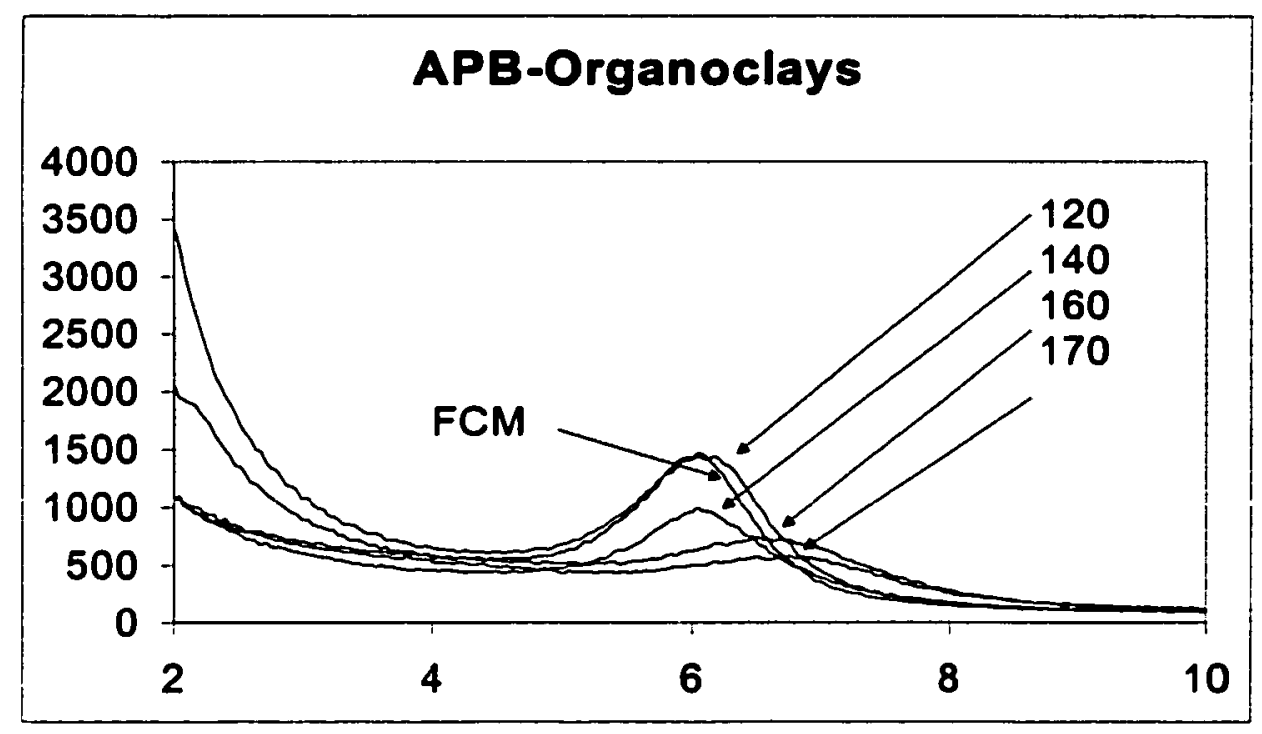

Figure 5. XRD pattern of APB organoclays 


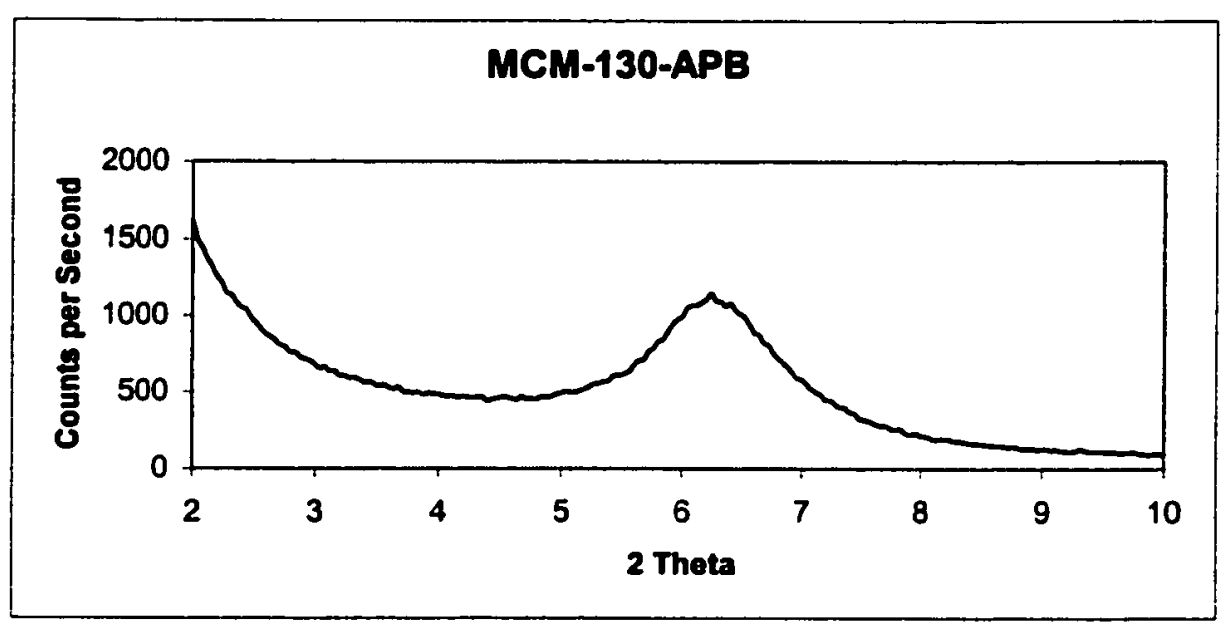

Figure 6. XRD pattern of MCM-130-APB organoclay

The XRD results were fairly close to our predictions. The fully charged clays and the clays that had a slight reduction in charge had the same d-spacing. The FCM, MCM120-APB, MCM-130-APB, and the MCM-140-APB organoclay all had the same dspacing $(1.46 \mathrm{~nm})$ as determined by looking at the maximum of the XRD pattern. The MCM-160-APB and MCM-170-APB organoclays both had a lower d-spacing at $1.37 \mathrm{~nm}$ and $1.31 \mathrm{~nm}$ respectively. This result was expected as the layer charge was low enough to allow the surfactant to lie closer to the clay surface.

$\mathrm{XRD}$ analysis of BAOD organoclays was expected to follow the same trend with respect to layer charge and d-spacing except the BAOD organoclays were expected to have a slightly larger $d$-spacing at all charges. The reason for the expected increase in spacing was the larger size of the BAOD ion. The XRD patterns for the BAOD organoclays are shown in Figure 7. 


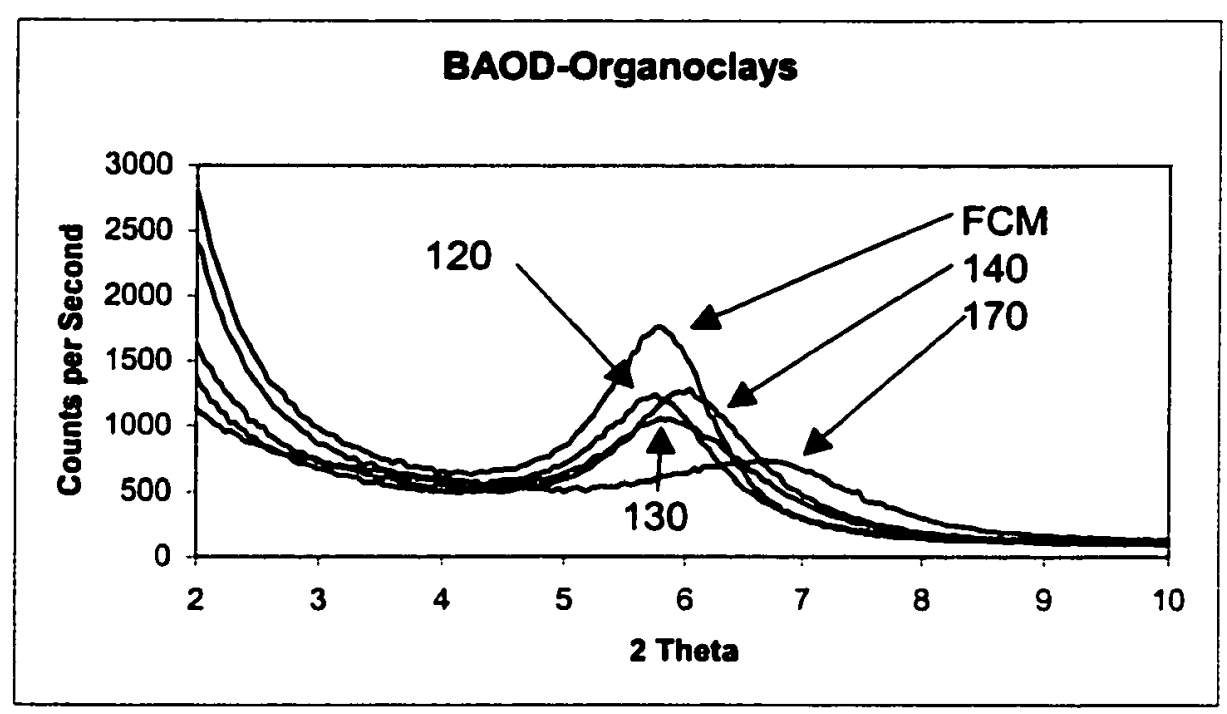

Figure 7. XRD pattern of BAOD organoclays

The results obtained from XRD analysis for these organoclays were very similar to those obtained for the APB organoclays. The layer spacing was slightly larger in these organoclays than in their APB counterparts. In the FCM-BAOD, MCM-120-BAOD, and MCM-130-BAOD clays the layer spacing was approximately the same and was $1.52 \mathrm{~nm}$. The MCM-140-BAOD clay had a lower d-spacing (1.46 nm) and the MCM-170-BAOD organoclay had an even lower d-spacing $(1.33 \mathrm{~nm})$.

The XRD results for both sets of organoclays proved quite convincingly that the organic ions were being exchanged for the inorganic ions. The organoclays had a larger d-spacing than the MCM-Na+ clays shown in Figure 4. The organoclays that had small charge reductions (i.e. 120,130) had d-spacings that were roughly the same as the FCM organoclays $(\sim 1.3 \mathrm{~nm})$. The clays where the charge was reduced further (i.e. 160,170$)$ the d-spacing of the organoclay started to decrease.

A relationship between d-spacing, lateral spacing between the surfactant ions, and clay dispersion in the final nanocomposite material is apparent from the data. For 
example the MCM-130 clays are among the group of higher d-spaced organoclays but have a lower charge than the FCM and 120 clays. This clay had the maximum lateral spacing between surfactant ions without losing any height in d-spacing. The MCM-130 clay performed the best in the preparation of polyimide nanocomposites. This suggests that a clay where the d-spacing and lateral spacing are both at a maximum (i.e the ratio of d-spacing to surface charge is maximized) may be the best case scenario when using aromatic surfactants. With the aromatic ions a reduction in d-spacing occurs but good dispersion is achieved when the charge is optimized. The enhanced properties of these reduced charge clays are related to lateral spacing and not just to increased d-spacing as in the case of aliphatic clays. The clays where the charge was greatly reduced (MCM160 and MCM-170) exhibited smaller inner layer height than the FCM organoclays. This may explain the poor dispersion behavior of these clays in polymeric matrices.

\subsection{XRD analysis of FCM-APB and MCM-APB/APB-BPDA films}

One of the tools used for determining clay dispersion in polymeric matrices is XRD. The XRD pattern gives information on the ordering of clay particles in the polyimide material. The XRD patterns for the variably charged 3\% MCM-APB/APBBPDA polyimide films are reported in Figures 8 and 9. All of the organoclays used in the film preparation were prepared from sodium treated clays and the organoclay was extracted for 8 hrs. The patterns were shown on two separate figures because the patterns were hard to distinguish when placed in the same figure. 


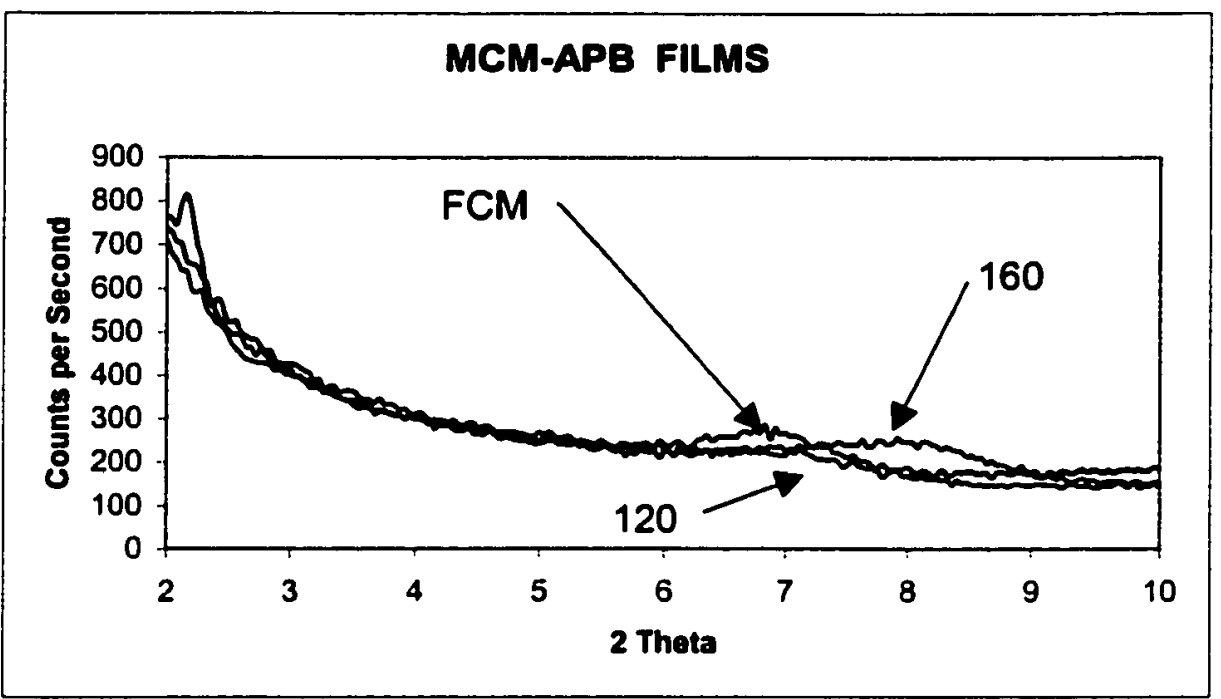

Figure 8. XRD pattern of MCM-APB/APB-BPDA hybrid films

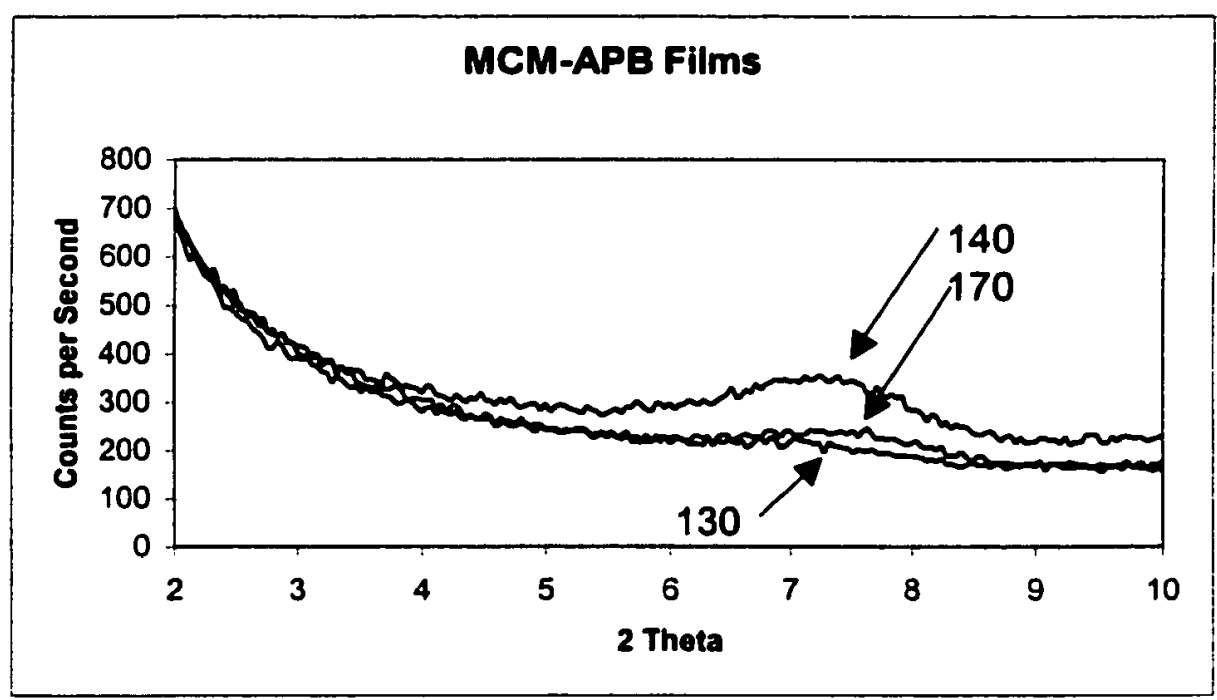

Figure 9. XRD pattem of MCM-APB/APB-BPDA hybrid films

The XRD patterns for the films reveal that in all of the polymer films there was some clay that was not exfoliated. There is no way to determine the percentage of clay that was ordered using XRD. The d-spacings for the organoclays after hybrid formation and curing were smaller than their respective organoclay before mixing with the polymer. This indicated that there was a partial collapse of some of the clay layers. This result was not expected with these high temperature clays and is not understood at this point. The 
organic surfactant material can withstand temperatures higher than the cure temperature of the polymer so it was unlikely that the collapse was due to surfactant degradation. A peak at $2 \theta=2.16^{\circ}$ indicated that the MCM-120-APB/APB-BPDA film had some intercalated clay particles. The MCM-130-APB did have a small peak but the existence of clay with this ordering is limited to a small amount of clay as revealed in the TEM micrograph. The remaining films all had peaks in the XRD pattern and the peaks were thought to represent larger amounts of agglomerated clay.

\subsection{XRD analysis of BAOD nanocomposite films}

The screening process of the variably charged organoclays continued by preparing films prepared with 3\% BAOD organoclays. The characterization of these materials included obtaining XRD patterns of these films. The XRD patterns are shown below in Figures 10 and 11. Two figures were used so the individual patterns could be distinguished.

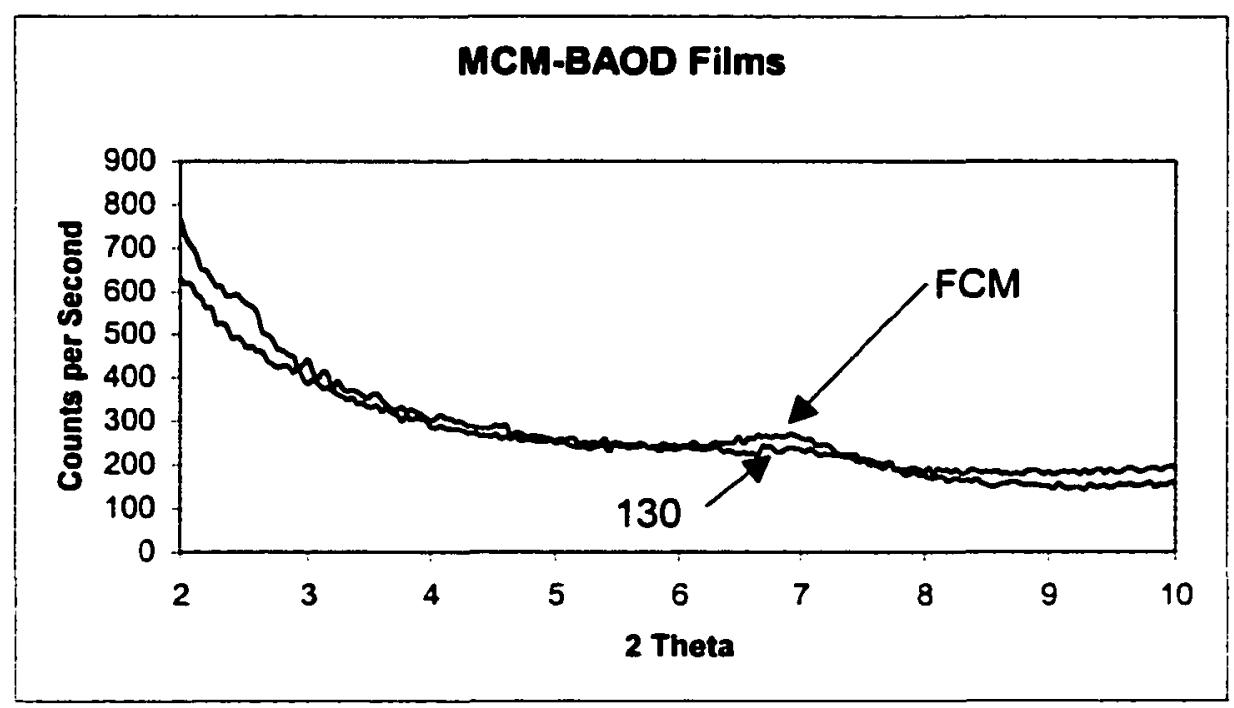

Figure 10. XRD pattern of MCM-BAOD/APB-BPDA hybrid films 


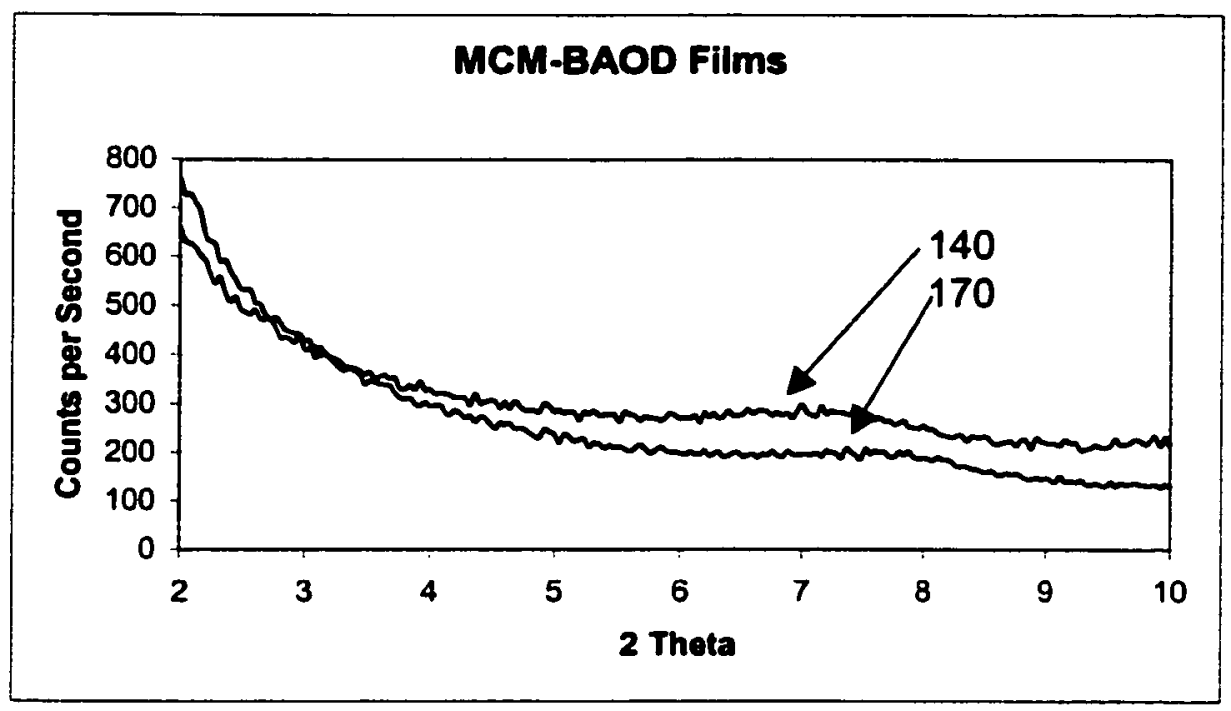

Figure 11. XRD pattern of MCM-BAOD/APB-BPDA hybrid films

Four 3\% loaded films were prepared with BAOD organoclays. The XRD patterns for all of the polyimide films exhibited a peak in the range scanned. This indicated that all the films contained ordered clay particles. The peak for the MCM-130-BAOD film was at the same angle as the peak for the FCM-BAOD/APB-BPDA film and the ordered particles in both systems had a d-spacing of $1.28 \mathrm{~nm}$. The lower charged clays had slightly lower d-spacings, which were $1.26 \mathrm{~nm}$ and $1.20 \mathrm{~nm}$ for the MCM-140BAOD/APB-BPDA and MCM-170-BAOD/APB-BPDA films, respectively. Again the percentage of the clay with this ordering cannot be determined from XRD data but when this data was coupled with the TEM micrographs it was determined that the best dispersion was obtained with the MCM-130-BAOD/APB-BPDA film.

3.7. XRD analysis of MCM-130-APB nanocomposite films After determining that the optimum charge for producing highly dispersable, high 
temperature organoclays it was of interest to further investigate the performance of the hybrid materials produced from these organoclays. The organoclay with the best dispersion in APB-BPDA was the organoclays prepared from MCM-130 clays. The preparation method for the organoclay was changed when additional analysis of these materials was performed. The lithium clay was used for the ion exchange reaction and the clay was extracted for 24 hours instead of 8 hours. The hybrid films were prepared in the same manner as the previously discussed films. Although the same preparation scheme was used, different concentrations $(3,5$, and $8 \%)$ of clay were added. The XRD patterns of the films are shown below in Figure 12.

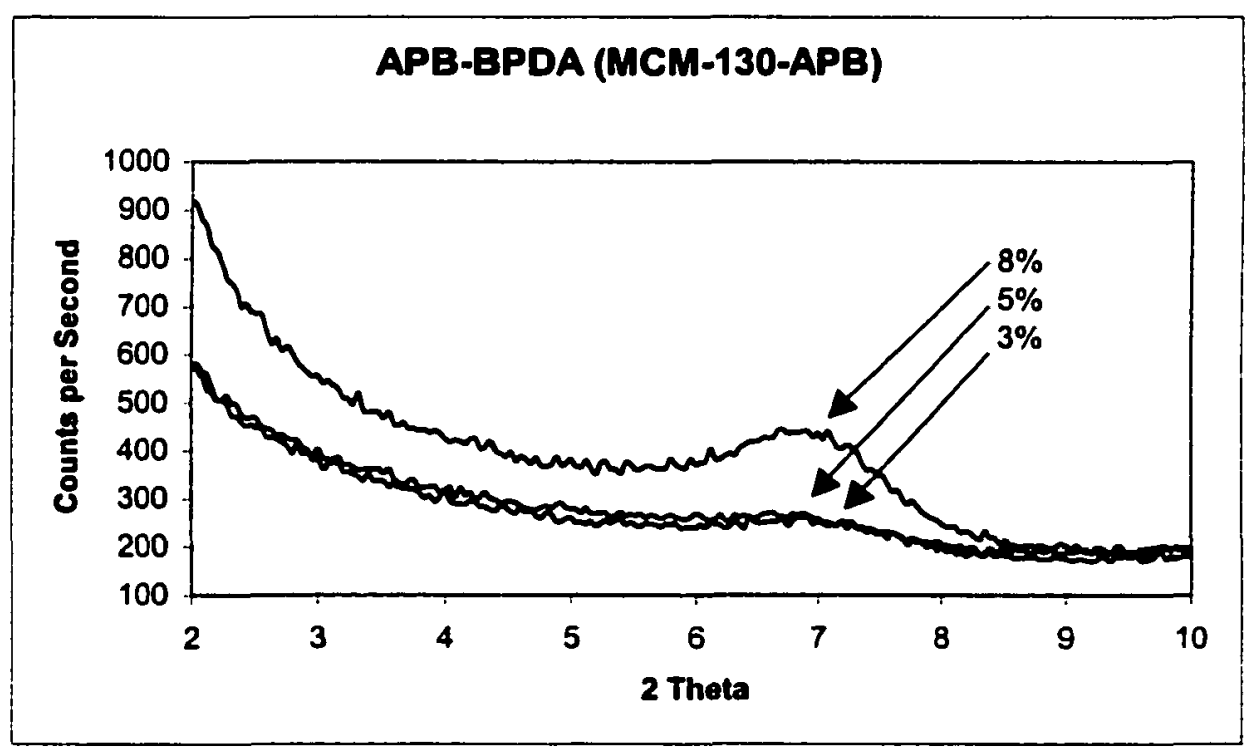

Figure 12. XRD pattern of MCM-130-APB/APB-BPDA hybrid films

All of the film samples exhibited a peak in the XRD pattern. The position of the peak was roughly the same and the maxima occurred at $2 \theta=6.92^{\circ}$. This is indicative of a dspacing of $1.28 \mathrm{~nm}$. This peak showed that there were some clay particles that did not completely exfoliate during hybrid preparation. The peak grew in intensity as the concentration was increased. Although claims of being able to determine concentration 
of oriented clay particles with XRD are not usually encouraged, these data seems to illustrate that a correlation can be made. No further investigation of this claim was performed. The data shown here indicates that there is some clay that maintains an oriented structure after the MCM-130-APB/APB-BPDA films were prepared via in-situ polymerization.

\subsection{XRD analysis of MCM-130-BAOD films}

The excellent dispersion of organoclay witnessed when MCM-130-APB organoclay was used prompted further investigation using the MCM-BAOD organoclays with the same charge. APB-BPDA films were prepared at different concentrations using the MCM-130-BAOD organoclays and the in-situ polymerization technique. The organoclays were also prepared from the lithium clays and were extracted for $24 \mathrm{hrs}$. The organoclays were used in hybrid formation and the hybrid mixtures were used to prepare films. The films were examined with XRD and the XRD patterns are shown in Figure 13.

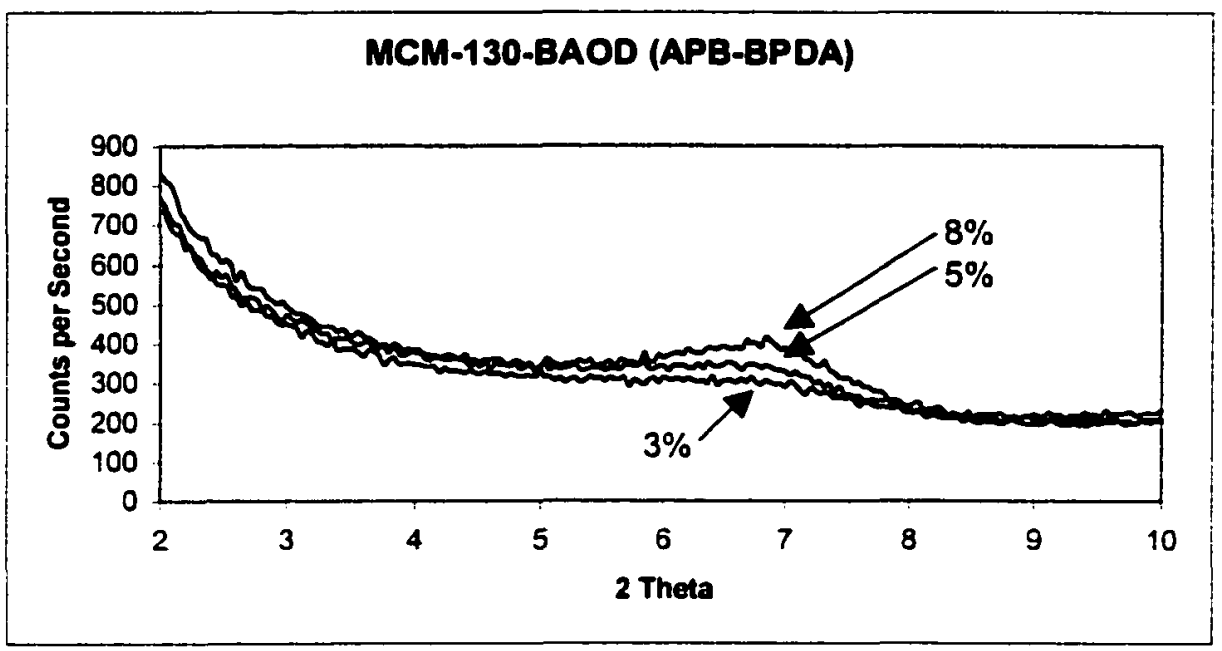

Figure 13. XRD pattern of MCM-130-BAOD/APB-BPDA hybrid films 
The XRD patterns for these films with varying concentrations of organoclay all exhibited a peak. The peak position was approximately the same in all of the patterns $\left(2 \theta=6.84^{\circ}\right)$. This indicated a d-spacing of $1.29 \mathrm{~nm}$. This represents a smaller $\mathrm{d}$-spacing than the neat organoclay. Some of the organoclay was not exfoliated and did have a reduced d-spacing after film formation. The peak grew in intensity as the concentration was increased in this system as well.

3.9. Mechanical properties of nanocomposite thin films

The organoclays were screened to determine a clay surface charge that would produce high quality nanocomposites with the chosen surfactant. These organoclays were used in the preparation of APB-BPDA hybrid films and mechanical data was collected for these films. The mechanical results reported in this section were measured in tensile tests. The films were cut and prepared for testing in the exact same way. The results reported in this section were measured on films containing $3 \%$ organoclay. These organoclays were prepared from sodium clays and the clays were only extracted for 8 hours. Further testing was done with the MCM-130-APB clay where lithium clays were used for the ion exchange and the clays were extracted for 24 hour. The results of those experiments are not in this section. The mechanical properties in this section were measured to determine if there was a pattern that could be related to the other characterization of these systems. Although small differences in levels of dispersion are hard to expose when looking at the bulk mechanical properties of a material, the data did reveal a pattern that is discussed below. 
The first three figures compare the modulus, percent elongation, and tensile strength of APB-BPDA nanocomposite films prepared with MCM-APB organoclays of various charges. The moduli of these materials are presented in Figure 14.

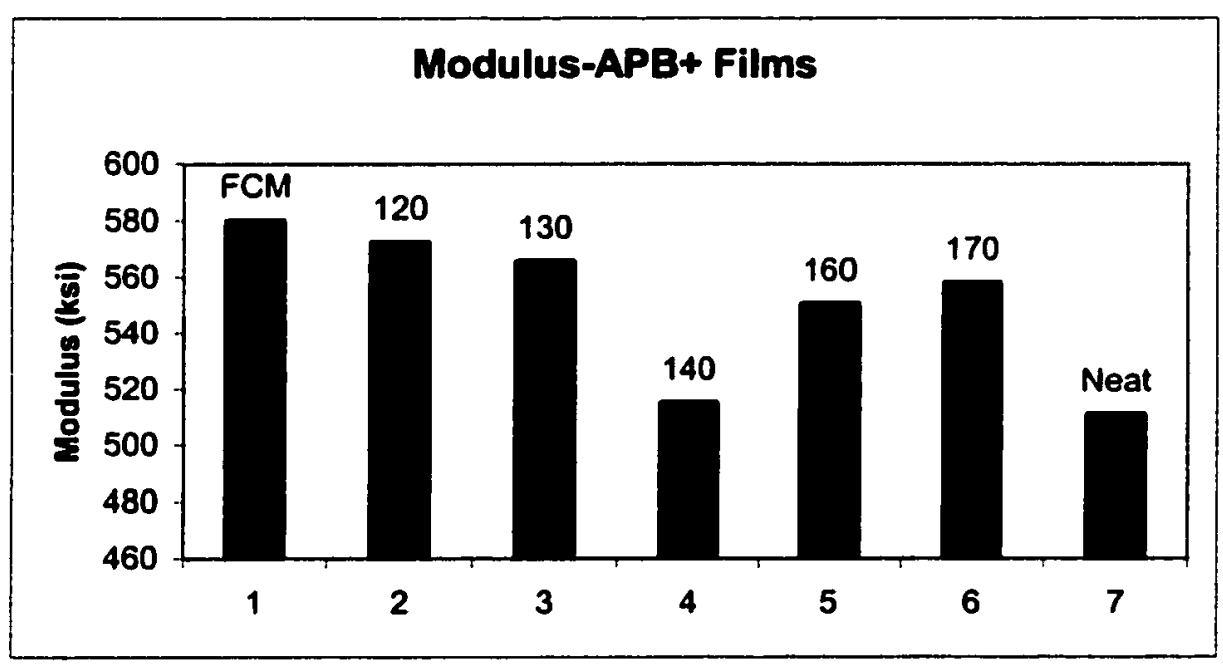

Figure 14. Modulus of APB organoclay/APB-BPDA hybrid films

The addition of clay increased the modulus relative to the neat polymer in all the films. The modulus decreased as the charge was reduced on the clay surface up to a point. The less charge present on the surface implied that there were less ions on the surface and of course there were less tethering points for the PAA as a result. Therefore, the reduction in modulus in the lower charged clays may be a result of less polymer attached to the clay surface. The film prepared with MCM-140-APB organoclay had the lowest modulus. Although the films up to this point had reduced moduli as the charge was lowered, when the charge was further reduced the materials started to have higher moduli. It was difficult to make a statement as to why the modulus data started to increase again in the MCM-160 and MCM-170 film. The MCM-130-APB organoclay was the clay that exhibited the best dispersion behavior according to the other characterization but no large change in the modulus was witnessed. 


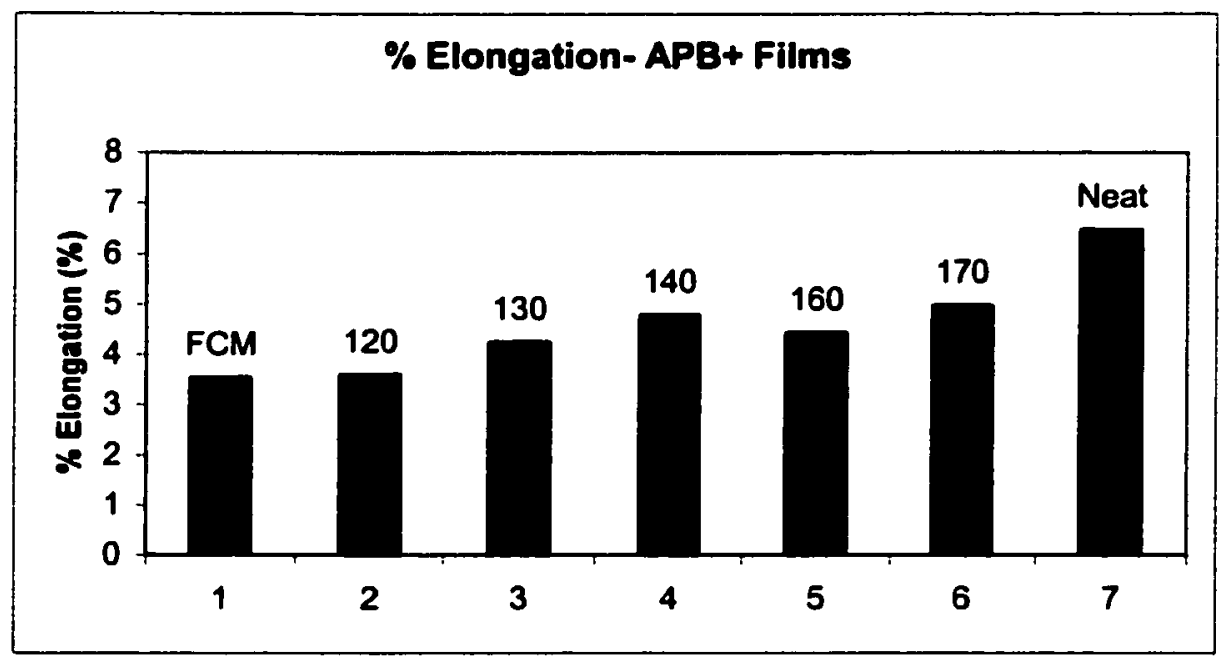

Figure 15. Elongation of APB organoclay/APB-BPDA hybrid films

The elongation properties of the MCM-APB/APB-BPDA films are shown in Figure 15.

These results showed that the addition of clay was detrimental to elongation as all the elongation values were below that of the neat polymer. The elongation values rise as the charge is reduced and the best results were seen in the MCM-170-APB film. This was an unexpected result, as the MCM-170-APB did not contain well-dispersed organoclay. The increase in percent elongation as the charge is reduced seemed to be a trend except the MCM-160-APB that does not fit the pattern. It is unclear why the elongation values increased as the charge was reduced. The tensile strength of these same materials are reported in Figure 16. There was a drop in strength going from the FCM-APB film to the MCM-120-APB film. As in the case of elongation, the strength of these materials increased as the charge was reduced and the MCM-170-APB film had the largest strength value. Again all of the hybrid films had lower strengths than the neat polyimide. 


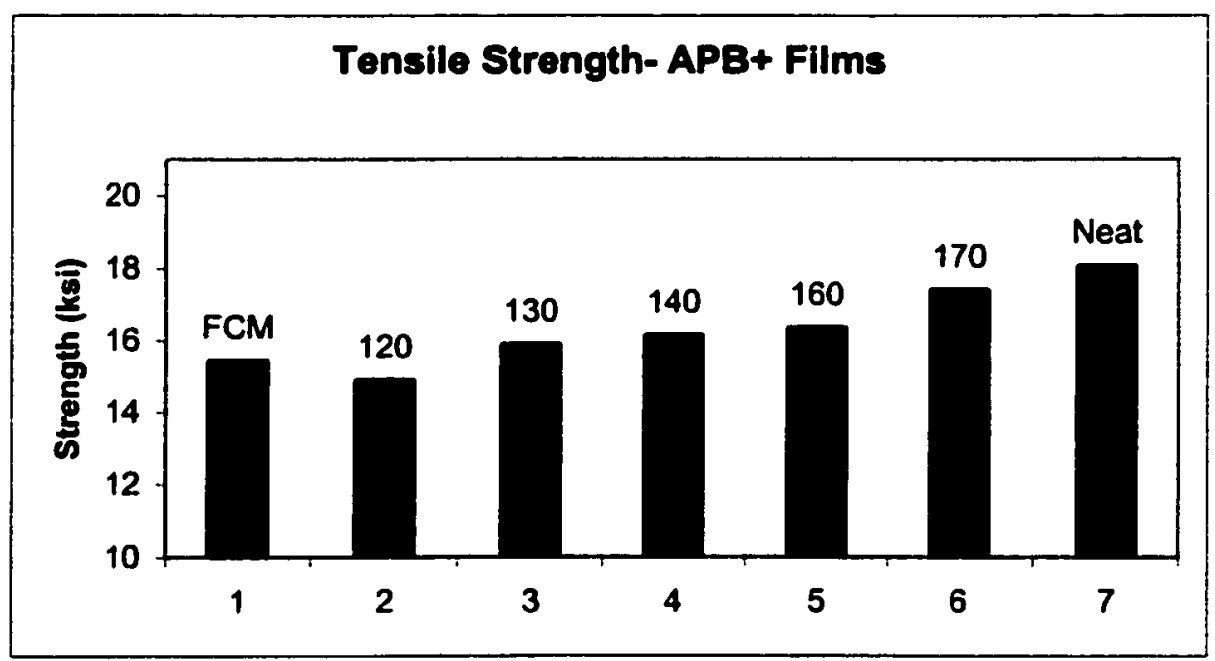

Figure 16. Strength of APB organoclay/APB-BPDA hybrid films

To further examine the relationship between layer charge, dispersion, and mechanical properties a series of films were prepared with BAOD organoclay. The BAOD surfactant was used to modify the surface of clay with four different layer charges. The films were prepared in the same manner using the same polyimide (APBBPDA) and the same clay loading as in the other films (3\%). The modulus data for these films are reported in Figure 17.

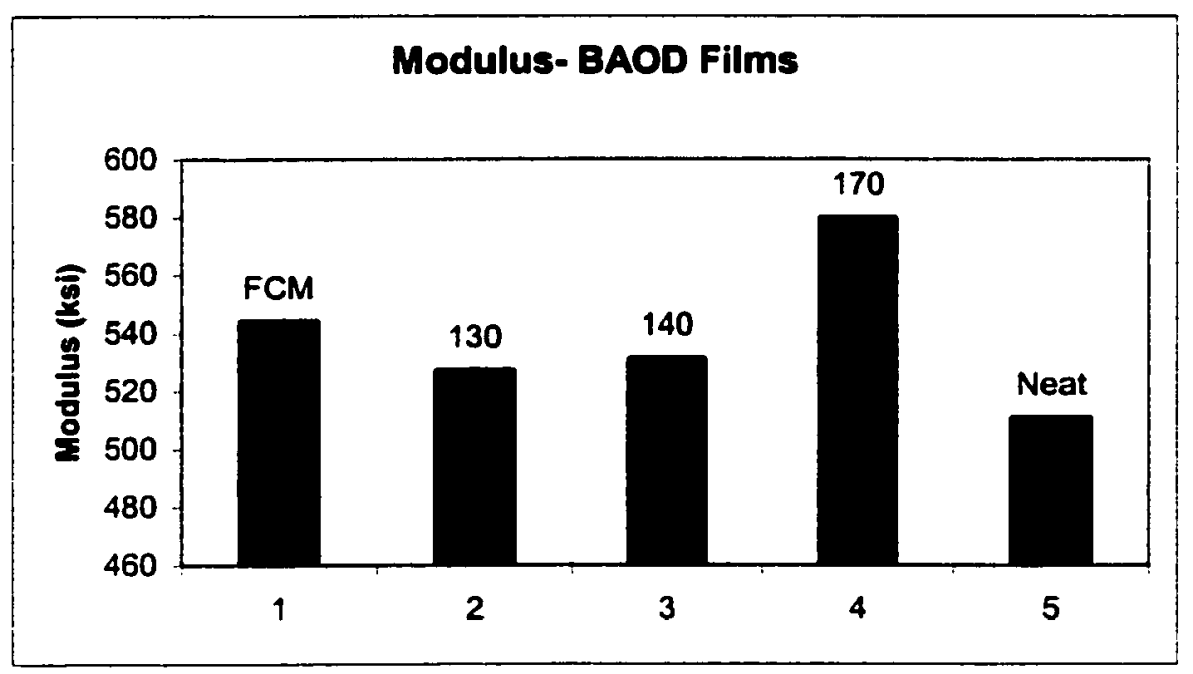

Figure 17. Modulus of BAOD organoclay/APB-BPDA hybrid films 
The modulus data were similar to the data obtained from the MCM-APB/APB-BPDA hybrids. There was an increase in the moduli relative to the neat polyimide in all the materials in which clay was introduced. The degree to which the modulus increased seemed to depend somewhat on the layer charge of the material. The modulus decreased when FCM-BAOD was changed to MCM-130-BAOD. The moduli of these materials then increased as the layer charge was further reduced. The MCM-170-BAOD was the film that exhibited the highest modulus. The elongation properties of the material seemed to follow the same trend as in the case of the MCM-APB materials. The results are shown in Figure 18.

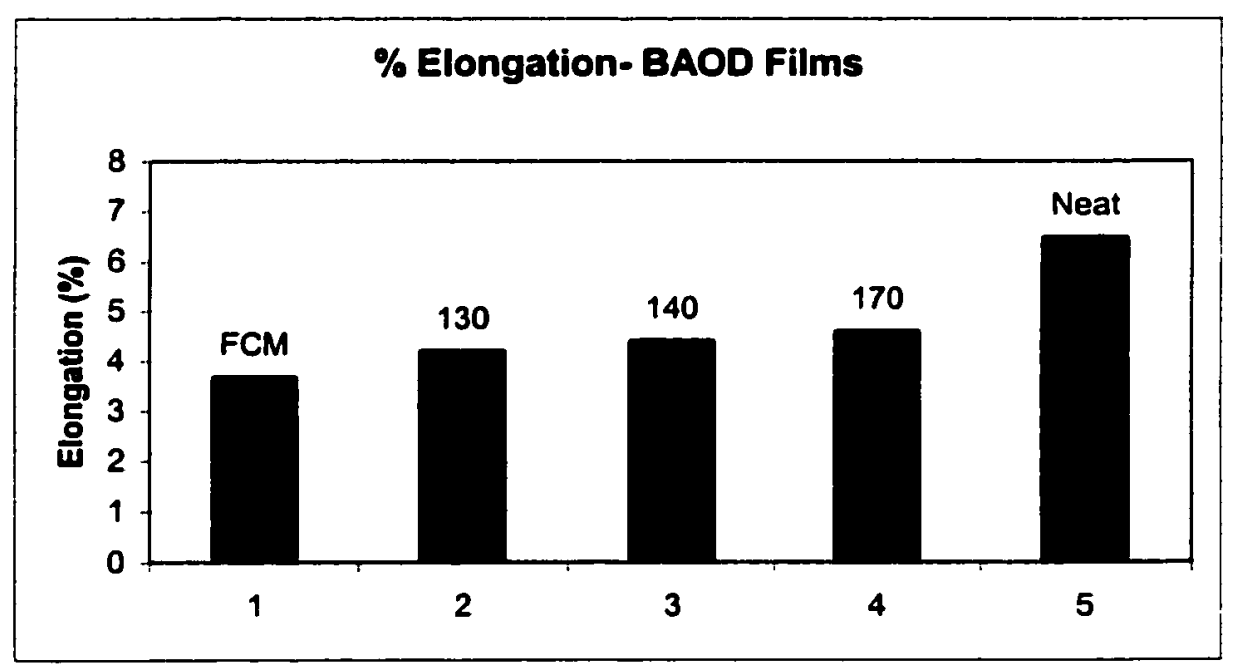

Figure 18. Elongation of BAOD organoclay/APB-BPDA hybrid films The elongation of these materials increased as the layer charge was reduced. This again was not a result of better dispersion when these clays were used. Other characterization showed that the lower charged clays did not disperse well. The MCM-170-BAOD organoclays were not as well dispersed as the MCM-130-BAOD organoclays and the clay was actually visible as small dots in the polyimide matrix. These mechanical results cannot be explained. The tensile strengths for these films are shown in Figure 19. 


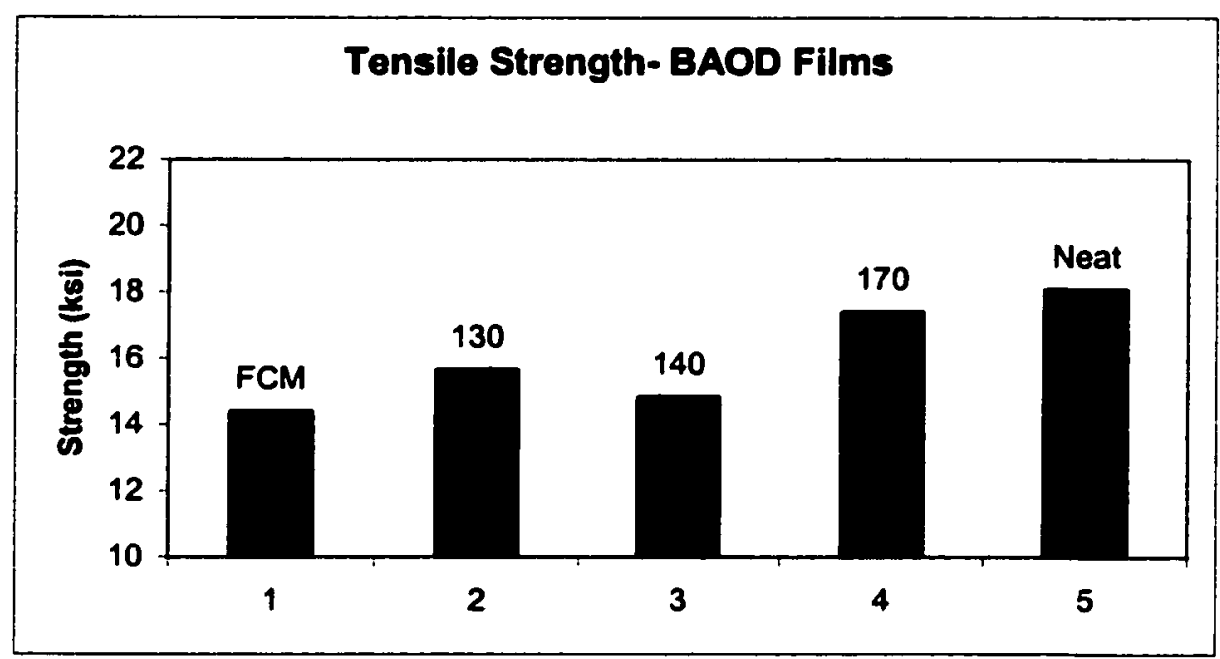

Figure 19. Strength of BAOD organoclay/APB-BPDA hybrid films

Aside from the MCM-140-BAOD film, the strength of these films increased as the layer charge was reduced. The highest strength besides that of the neat material belonged to the MCM-170-BAOD/APB-BPDA film.

The mechanical properties of these films showed that the modulus increases and the elongation and strength decreases with the addition of organoclay. The degree to which the values change has some dependence on the dispersion which in turn is related to the surface charge. The relationship between the dispersion of the organoclay and the mechanical properties of nanocomposite films is not completely understood at this point.

\subsection{Mechanical properties of MCM-130-APB/APB-BPDA films}

The MCM-130 clays were used to prepare higher clay concentration films $(3,5$, $8 \%)$ and the films were characterized for mechanical properties. In these experiments the organoclay was kept as the lithium clay, exchanged with the APB or BAOD surfactant, and extracted with water for $\mathbf{2 4} \mathrm{hrs}$. The clay was then lyophilized and the lyophilized 
organoclay was used in the preparation of hybrid films at the three different clay loadings via in-situ polymerization.

The modulus of a material in which high modulus filler is added is expected to increase. The amount of increase should be directly linked to the concentration and aspect ratio of the filler material. The clay materials are a high aspect ratio material when properly dispersed and have in some instances increased the modulus of polymeric materilals ${ }^{1}$. The modulus of the 3, 5, and $8 \%$ films are shown in Figure 20.

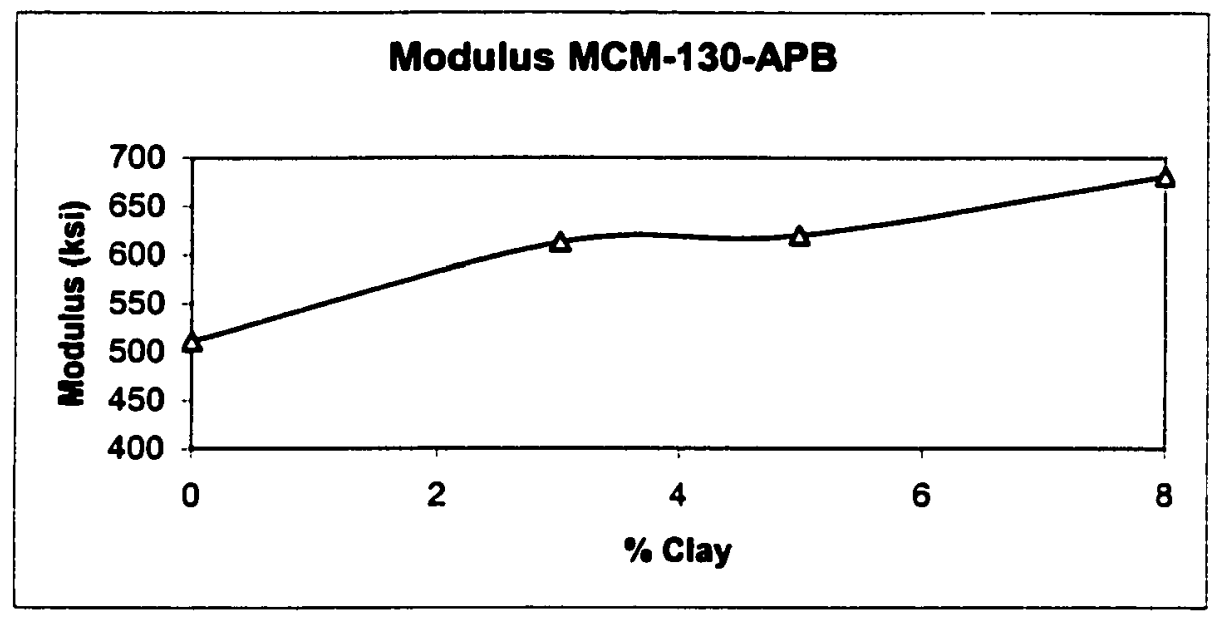

Figure 20. Modulus of MCM-130-APB/APB-BPDA hybrid films

As expected the modulus increased quite considerably with the addition of the organoclay. The increase was aiso proportional to the amount of clay that was added. The modulus increased significantly with only a $3 \%$ addition of clay (100 ksi). There was a continual increase in the modulus as the clay concentration was increased up through $8 \%$.

The addition of an increased concentration of clay particles to polyimides decreased strengths in Chapter III. It is suspected that very good dispersion is necessary to keep the strength values at or above that of the neat polymer. When agglomerates are 
present then the strength will most likely decrease. The strength values for three concentrations of MCM-130-APB organoclay in APB-BPDA are shown in Figure 21.

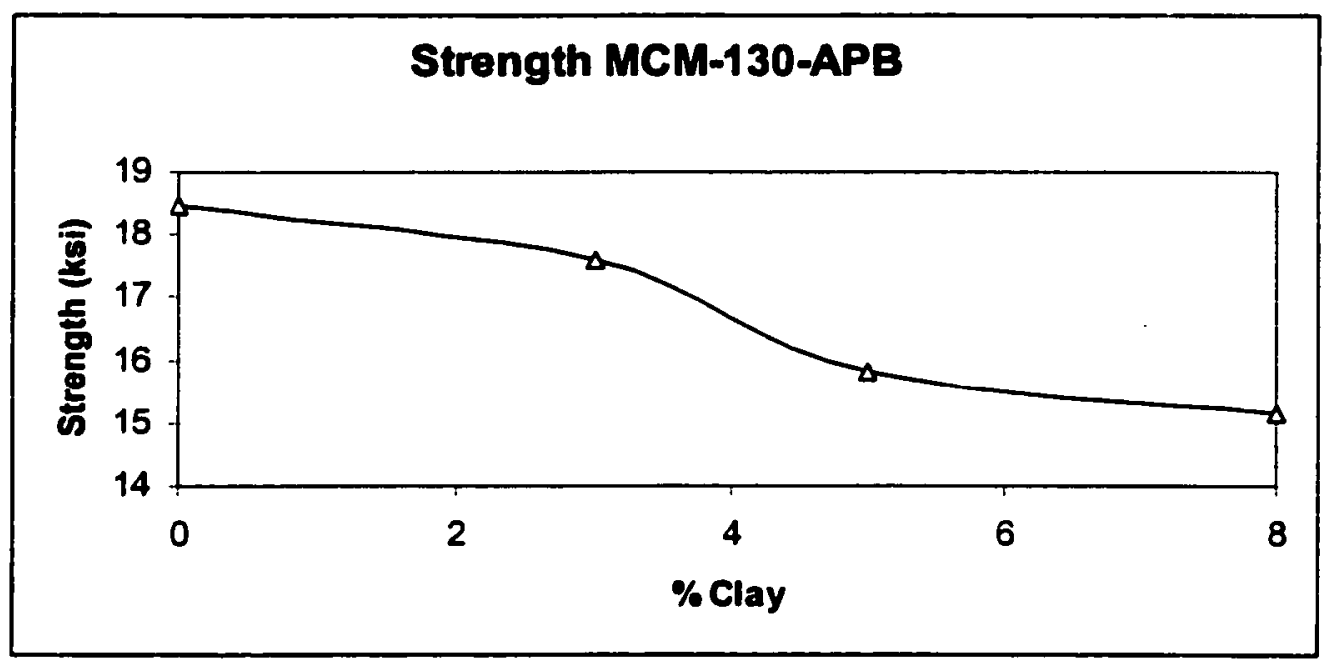

Figure 21. Strength of MCM-130-APB/APB-BPDA hybrid films

The strength values did decrease as the clay concentration was increased as seen in this figure. The decrease is proportional to clay concentration and it is not known whether it is an effect of increased agglomeration in the system or an increase in the immobilized region of polymer around the clay particles.

The elongation properties of nanocomposite materials usually follow the same trend as the strength values. The elongation will usually decrease at a rate that is proportional to the increase in the amount of clay that is added. This is especially true if there are clay agglomerates present in the system. The data for percent elongation is shown in Figure 22 and is consistent with predictions as well as with previous work. The elongation properties of this material decrease as the clay concentration is increased. 


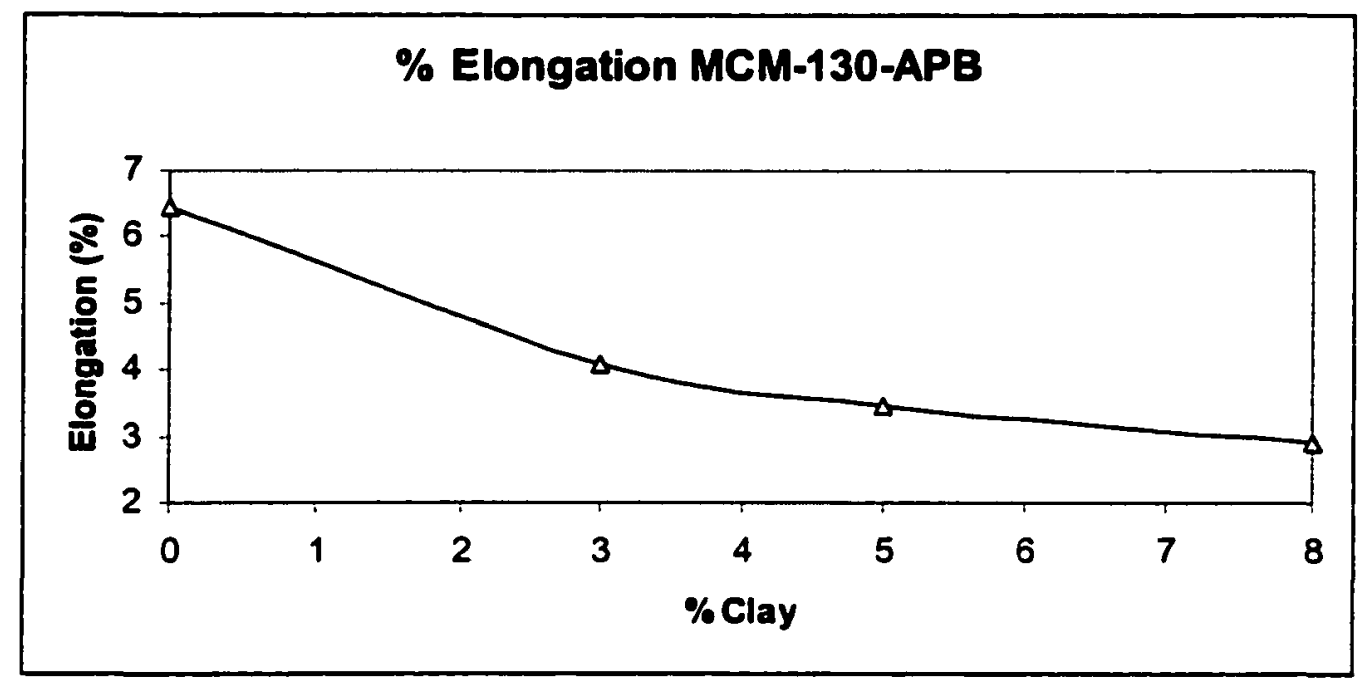

Figure 22. Elongation of MCM-130-APB/APB-BPDA hybrid films

3.11. Mechanical Properties of MCM-130-BAOD/APB-BPDA hybrid films

APB-BPDA films were prepared with 3, 5, and 8\% loadings of MCM-130-BAOD organoclay. The organoclay was prepared from the MCM-130-Li clay and was extracted for $24 \mathrm{hrs}$ prior to use. The lyophilized clay was used in hybrids that were prepared via in-situ polymerization. The mechanical properties of these materials were measured and the results are shown below. The modulus data is shown in Figure 23.

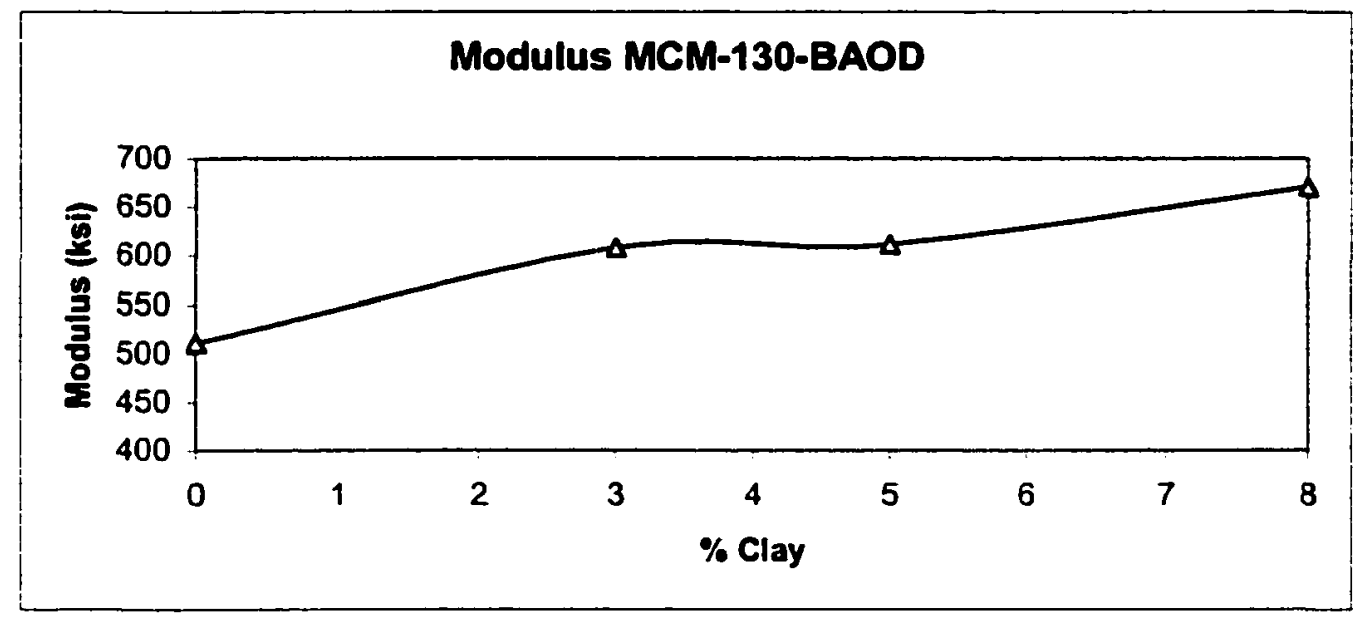

Figure 23. Modulus of MCM-130-BAOD/APB-BPDA hybrid films 
The modulus of the material increased proportionally to the amount of clay added. This is consistent with the MCM-130-APB system discussed as well as previous polyimide nanocomposite work ${ }^{2}$. The increase in modulus indicated that there was some clay that was well dispersed in the polyimide matrix. The strength data for the same system is shown below in Figure 24.

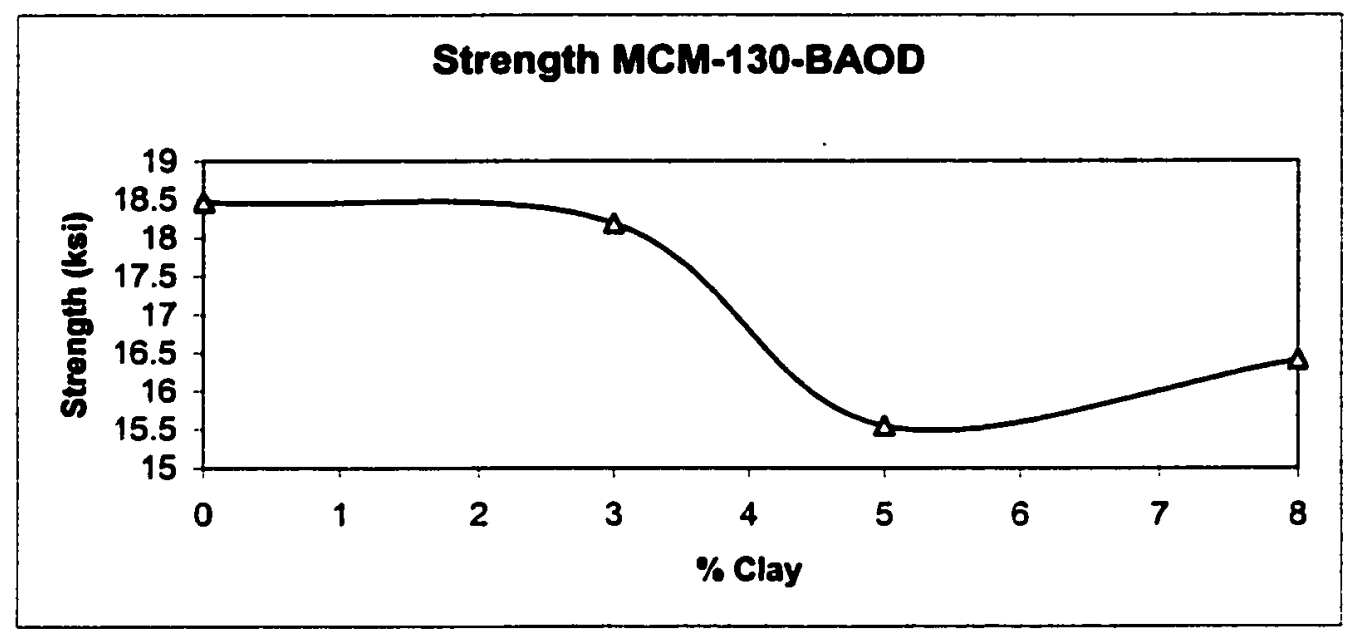

Figure 24. Strength of MCM-130-BAOD/APB-BPDA hybrid films

This data is somewhat different than other data collected for these types of systems. The strength of the neat material is almost the same as the strength for the $3 \%$ material. The film with $5 \%$ clay loading had a noticeable decrease in strength but then the $8 \%$ film had higher strength than the $\mathbf{5 \%}$ film. It is difficult to determine if this is an artifact or some unique feature is causing this. The elongation properties for this system are shown in Figure 25. This data is consistent with other elongation data obtained for these types of systems. The percent elongation value decreases as the clay concentration increases and presumably the concentration of clay agglomerates increases. 


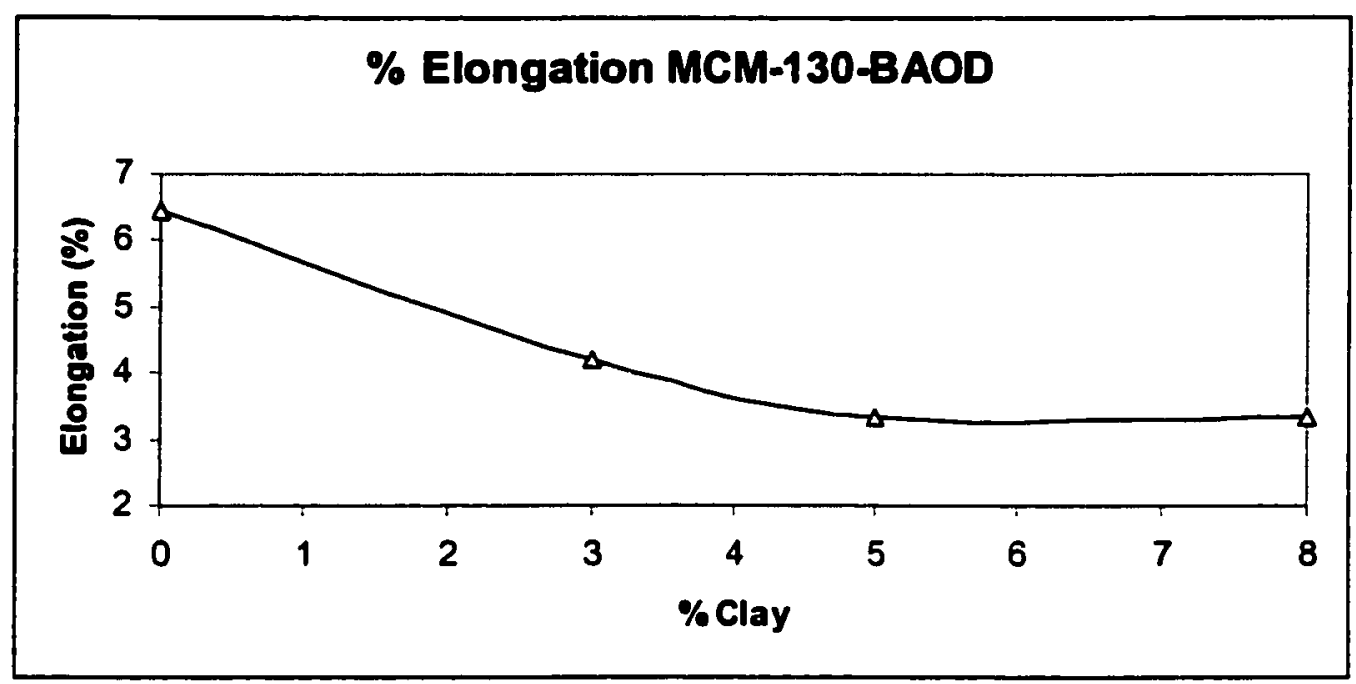

Figure 25. Elongation of MCM-130-BAOD/APB-BPDA hybrid films

\subsection{Thermal analysis of organoclays}

The preparation of nanocomposite materials that contain a high level of clay dispersion depends on a number of features. One of the most important features is the thermal stability of the surfactant. There are usually negative side effects in the material if the surfactant degrades during processing. Aromatic surfactant ions were chosen to replace the conventional aliphatic ions in hopes of creating a more thermally stable surfactant. The organoclays that were prepared with APB and BAOD were characterized with TGA. The results of this study and the TGA results obtained from SCPX-2003 are presented below in Table 1 . 


\begin{tabular}{|c|c|c|c|c|c|}
\hline & FCM-APB & FCM-BAOD & MCM-130-APB & $\begin{array}{l}\text { MCM-130- } \\
\text { BAOD }\end{array}$ & SCPX-2003 \\
\hline \multicolumn{6}{|l|}{ Heating Profile } \\
\hline $\begin{array}{l}20{ }^{\circ} \mathrm{C} / \mathrm{min} \text { to } 100^{\circ} \mathrm{C} \\
30 \mathrm{~min} \text { hold at } 100^{\circ} \mathrm{C} \\
2.5^{\circ} \mathrm{C} / \mathrm{min} \text { to } 700^{\circ} \mathrm{C}\end{array}$ & & & & & $\begin{array}{l}\text { Air- } 233^{\circ} \mathrm{C} \\
\text { Nitrogen- } 233^{\circ} \mathrm{C}\end{array}$ \\
\hline $\begin{array}{l}20^{\circ} \mathrm{C} / \mathrm{min} \text { to } 100^{\circ} \mathrm{C} \\
30 \mathrm{~min} \text { hold at } 100^{\circ} \mathrm{C} \\
2.5^{\circ} \mathrm{C} / \mathrm{min} \text { to } 200^{\circ} \mathrm{C} \\
1 \mathrm{hr} \text { hold at } 200^{\circ} \mathrm{C} \\
2.5^{\circ} \mathrm{C} / \mathrm{min} \text { to } 700^{\circ} \mathrm{C}\end{array}$ & $\begin{array}{l}\text { Air- } 510^{\circ} \mathrm{C} \\
\text { Nitrogen- } 518^{\circ} \mathrm{C}\end{array}$ & $\begin{array}{l}\text { Air- } 469^{\circ} \mathrm{C} \\
\text { Nitrogen- } 444^{\circ} \mathrm{C}\end{array}$ & $\begin{array}{l}\text { Air- } 589^{\circ} \mathrm{C} \\
\text { Nitrogen- } 527^{\circ} \mathrm{C}\end{array}$ & $\begin{array}{l}\text { Air- } 484^{\circ} \mathrm{C} \\
\text { Nitrogen- } 395^{\circ} \mathrm{C}\end{array}$ & $\begin{array}{l}\text { Air- } 200^{\circ} \mathrm{C} \\
\text { Nitrogen- } 200^{\circ} \mathrm{C}\end{array}$ \\
\hline $\begin{array}{l}20{ }^{\circ} \mathrm{C} / \mathrm{min} \text { to } 100^{\circ} \mathrm{C} \\
30 \mathrm{~min} \text { hold at } 100^{\circ} \mathrm{C} \\
2.5^{\circ} \mathrm{C} / \mathrm{min} \text { to } 250^{\circ} \mathrm{C} \\
1 \mathrm{hr} \text { hold at } 250^{\circ} \mathrm{C} \\
2.5^{\circ} \mathrm{C} / \mathrm{min} \text { to } 700^{\circ} \mathrm{C}\end{array}$ & $\begin{array}{l}\text { Air- } 456^{\circ} \mathrm{C} \\
\text { Nitrogen- } 417^{\circ} \mathrm{C}\end{array}$ & $\begin{array}{l}\text { Air- } 408^{\circ} \mathrm{C} \\
\text { Nitrogen- } 405^{\circ} \mathrm{C}\end{array}$ & $\begin{array}{l}\text { Air- } 557^{\circ} \mathrm{C} \\
\text { Nitrogen- } 564^{\circ} \mathrm{C}\end{array}$ & $\begin{array}{l}\text { Air- } 431^{\circ} \mathrm{C} \\
\text { Nitrogen- } 447^{\circ} \mathrm{C}\end{array}$ & $\begin{array}{l}\text { Air- } 231^{\circ} \mathrm{C} \\
\text { Nitrogen- } 218^{\circ} \mathrm{C}\end{array}$ \\
\hline $\begin{array}{l}20^{\circ} \mathrm{C} / \mathrm{min} \text { to } 100^{\circ} \mathrm{C} \\
30 \mathrm{~min} \text { hold at } 100^{\circ} \mathrm{C} \\
2.5^{\circ} \mathrm{C} / \mathrm{min} \text { to } 300^{\circ} \mathrm{C} \\
1 \mathrm{hr} \text { hold at } 300^{\circ} \mathrm{C} \\
2.5^{\circ} \mathrm{C} / \mathrm{min} \text { to } 700^{\circ} \mathrm{C}\end{array}$ & $\begin{array}{l}\text { Air- } 525^{\circ} \mathrm{C} \\
\text { Nitrogen- } 487^{\circ} \mathrm{C}\end{array}$ & $\begin{array}{l}\text { Air- } 443^{\circ} \mathrm{C} \\
\text { Nitrogen- } 4222^{\circ} \mathrm{C}\end{array}$ & $\begin{array}{l}\text { Air- } 553^{\circ} \mathrm{C} \\
\text { Nitrogen- } 540^{\circ} \mathrm{C}\end{array}$ & $\begin{array}{l}\text { Air- } 434^{\circ} \mathrm{C} \\
\text { Nitrogen- } 462{ }^{\circ} \mathrm{C}\end{array}$ & $\begin{array}{l}\text { Air- Not done } \\
\text { Nitrogen- } 2311^{\circ} \mathrm{C}\end{array}$ \\
\hline
\end{tabular}

Table 2. TGA $5 \%$ weight loss values of organoclays.

It appears from the data presented here that both the APB organoclays and the BAOD organoclays were able to withstand the temperatures that are reached in polyimide preparation. The final hold temperature in the polyimide cure cycle is $300^{\circ} \mathrm{C}$. All of the aromatic organoclays had $5 \%$ weight loss temperatures that were higher than $300{ }^{\circ} \mathrm{C}$ regardless of heating profile. It was also interesting that the $5 \%$ weight loss temperature was higher for the APB organoclays than the BAOD clays in all of the trials. The SCPX2003 organoclay was not thermally stable at temperatures near $300^{\circ} \mathrm{C}$ and actually lost weight below $250^{\circ} \mathrm{C}$. The isothermal holds at 200,250 , and $300^{\circ} \mathrm{C}$ for the aromatic organoclays did cause some weight loss at the hold temperatures but the weight loss was not significant. Thus, all of the aromatic clays that were tested here are considered high temperature. 


\subsection{Determination of layer charge using atomic absorption}

The preparation of organoclays with aromatic surfactants that will disperse in polyimides has been the focus of this research. The level to which the organoclays will disperse is related to surface charge. To determine the optimum layer charge for the two surfactants used in this experiments, a number of organoclays were prepared and placed in APB-BPDA. In order to compare the clay dispersion with the charge on the clay surface, the cation exchange capacity (CEC) of some of the reduced charge clays were determined. The CEC was determined by measuring the exchangeable lithium as described in Section $2.8^{14}$. The CEC of the reduced charge clays was determined by comparing the amount of lithium that was present on the reduced charge clays with the exchangeable lithium in the FCM clay. The CEC was known for the FCM clay and thus the reduced charge clay CEC could be determined because the exchangeable lithium values were directly proportional. The CEC values were calculated and are presented in Table 2.

\begin{tabular}{|c|c|c|}
\hline Clay & $\begin{array}{c}\text { Total Lithium } \\
\text { Concentration } \\
\text { (ppm) }\end{array}$ & $\begin{array}{c}\text { CEC } \\
(\mathrm{meq} / \mathrm{g})\end{array}$ \\
\hline & & \\
\hline MCM-150 & 22.8 & 0.40 \\
\hline MCM-140 & 29.3 & 0.51 \\
\hline MCM-130 & 32.5 & 0.57 \\
\hline MCM-120 & 33 & 0.58 \\
\hline FCM & 51.3 & 0.90 \\
\hline
\end{tabular}


The data showed that the higher heating temperatures yielded clays with lower CEC values. This is what was expected and the results can be used when designing organoclays in the future.

\subsection{TEM analysis of nanocomposite materials}

TEM is a fundamental tool used to understand the dispersion behavior of particles in nanocomposite materials. A wealth of information can be obtained about nanocomposite materials if pictures are taken of the sample at different magnifications. The sizes of the particles can be calculated from these micrographs and the dispersion level can be estimated. Three micrographs were taken for every sample that was examined in this section at three different magnifications (3400x, 7200x, and 22000x). The data obtained at these different magnifications were used to discuss the dispersion of clay particles.

TEM data was collected for various nanocomposite preparations including those prepared with organoclays modified with the dihydrochloride salt of APB. TEM was used to compare films with differently charged MCM-APB organoclays as well as films with different concentrations of the MCM-130-APB organoclay. The micrographs obtained for MCM-130-APB organoclay placed in APB-BPDA at a loading of 3\% are shown below. 

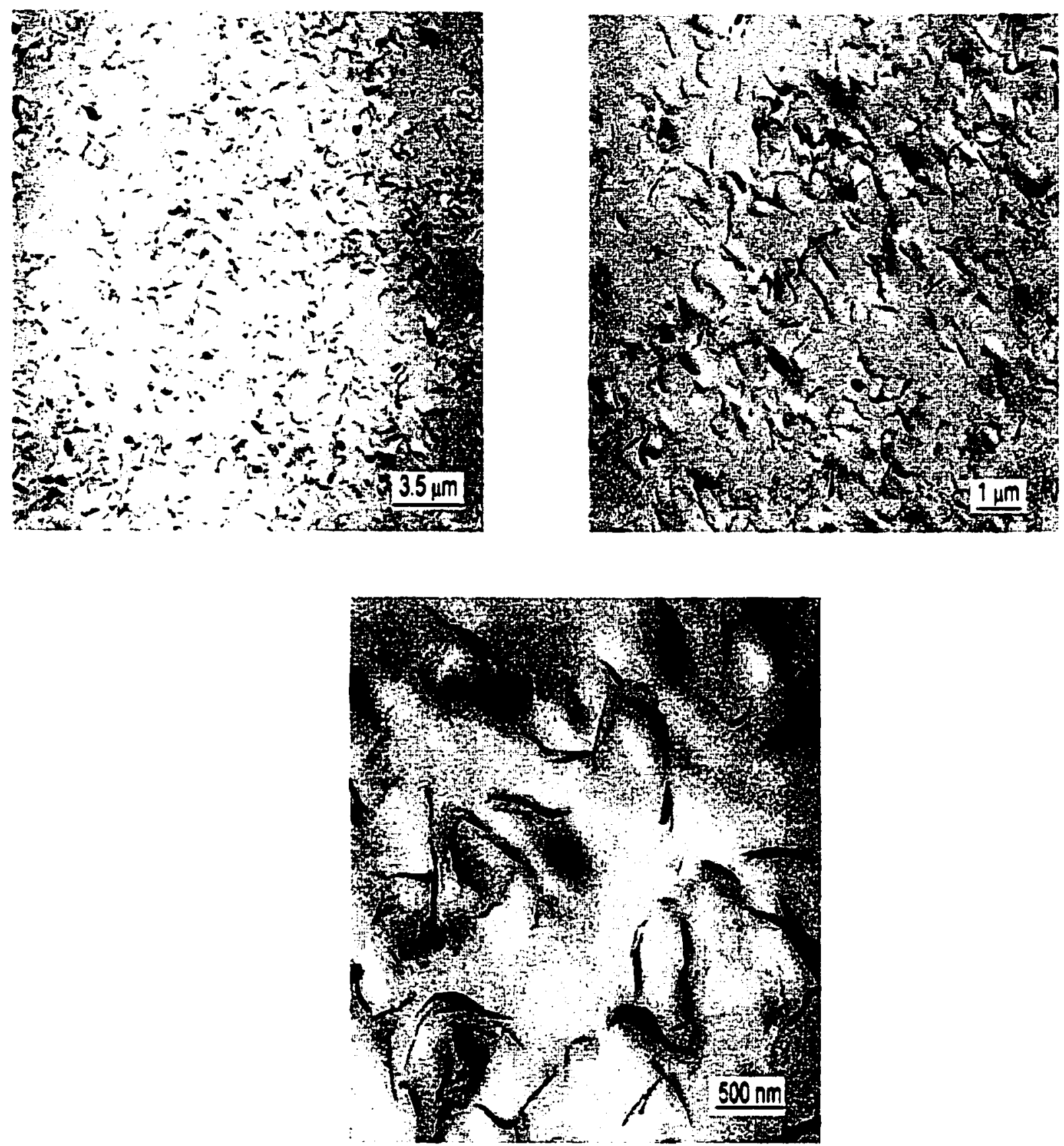

Figure 26. TEM micrographs of 3\% MCM-130-APB/APB-BPDA nanocomposite film

These TEM micrographs indicated that the clay particles were well dispersed. The level of clay cispersion in these films is relatively outstanding. The micrograph taken at the lowest magnification shows that there is homogeneous dispersion throughout the entire film. There are regions where small agglomerates are present but they are very few in 
number. The black smears most likely represent particles that were not completely perpendicular to the plane of the microtoming when the TEM sample was prepared. The micrographs taken at higher magnification further illustrate the great dispersion of the particles. The micrograph taken at $7200 x$ showed that there were not many agglomerates. The micrograph taken at $22000 x$ shows that the sizes of the few agglomerates were small and usually consists of only a few particles stuck together. The particles range in size from $\mathbf{2 0 0 - 7 0 0 ~} \mathrm{nm}$ in length and are immeasurably small in thickness. The micrographs shown here clearly indicate that the MCM-130-APB organoclay dispersed to nanoscopic dimensions in APB-BPDA at a $3 \%$ loading level.

The concentration of clay is an important factor in preparing nanocomposites with clay particles in polymer matrices. To examine the effects that raising the clay concentration had on clay dispersion in the polyimide film, the clay concentration was raised to 5\%. The micrographs presented below are taken of an APB-BPDA polymer film prepared with 5\% MCM-130-APB.
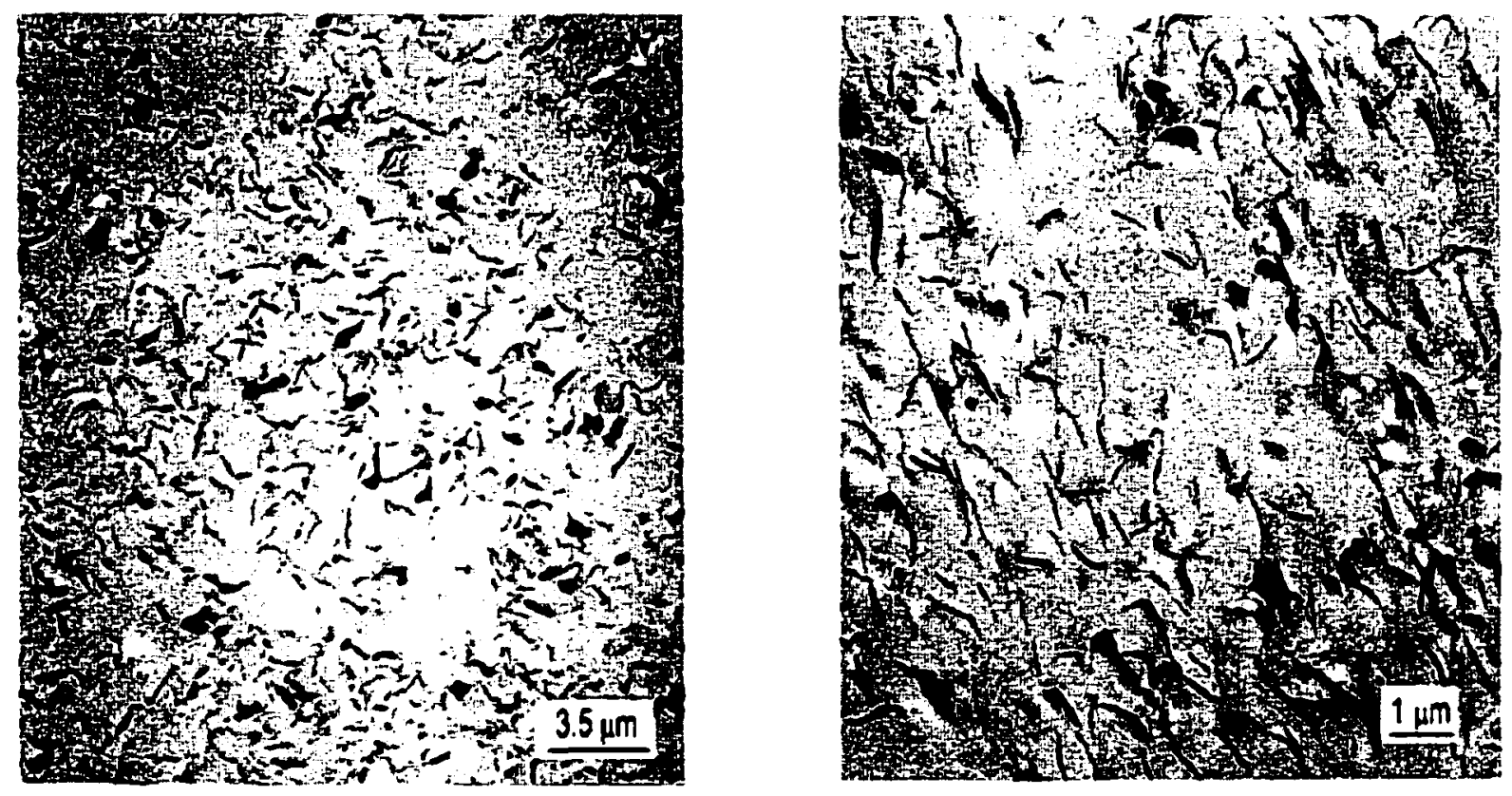


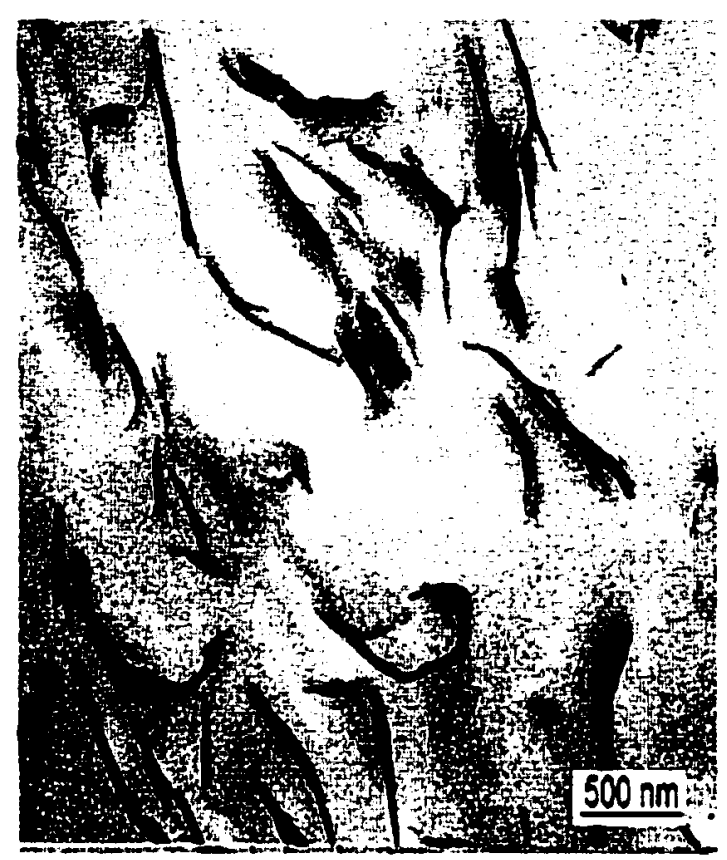

Figure 27. TEM micrographs of 5\% MCM-130-APB APB-BPDA nanocomposite film

The micrographs shown here contain the same organoclay as in the previous example and again show good dispersion. There are more individual clay particles in each micrograph due to the increased concentration. It was noticeable at the higher magnifications that there were less single clay particles and more bundles of clay particles at this higher concentration. Although they exist, the particles or bundles are homogenously dispersed as seen in the lower magnification films. There are no large areas of agglomeration in these films indicating good dispersion of clay in these samples.

The concentration was raised again in the next sample examined with TEM. In this sample MCM-130-APB was incorporated into APB-BPDA at $8 \%$ loading. The TEM micrographs for this material are presented in Figure 28. 

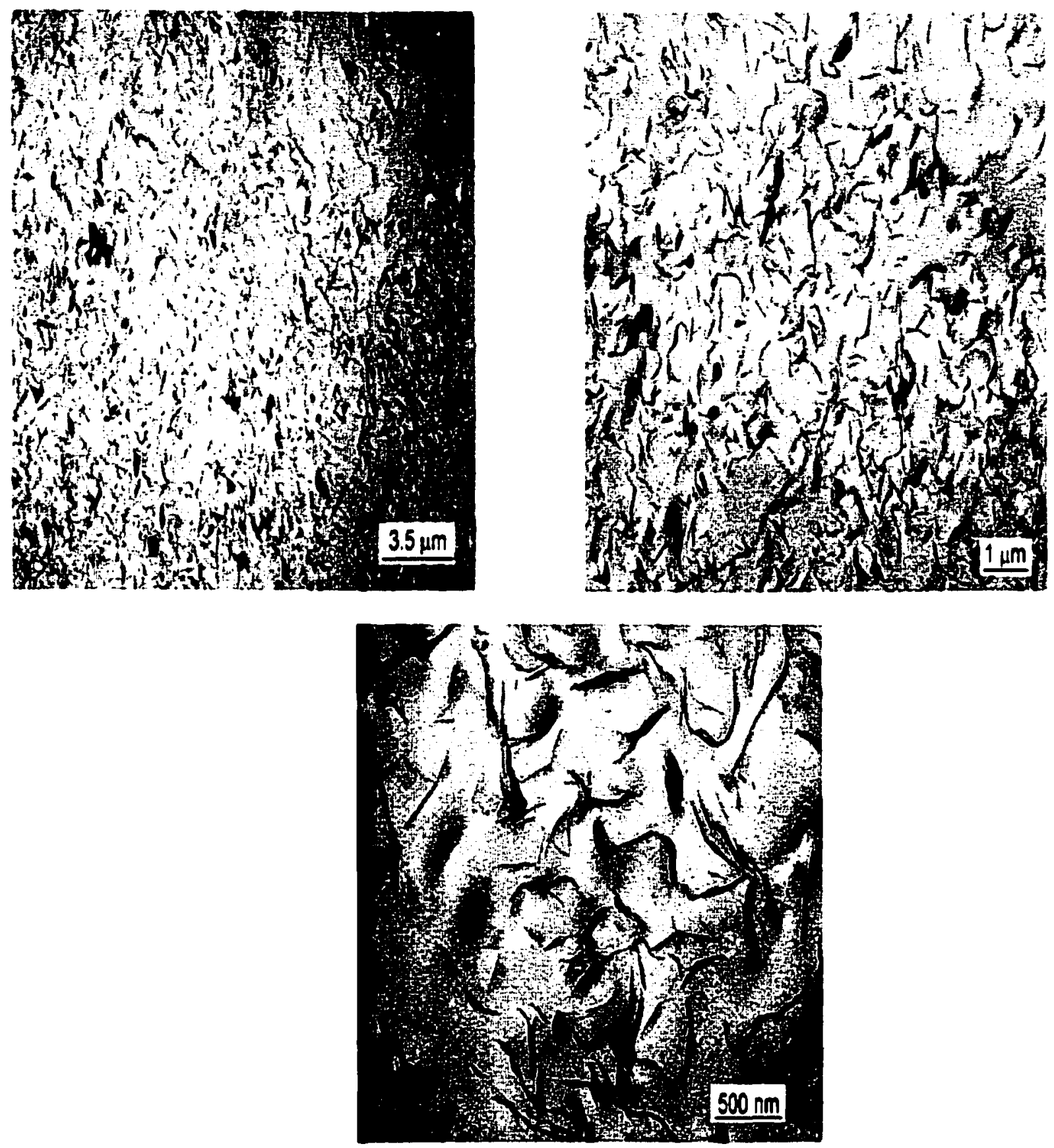

Figure 28. TEM micrographs of $8 \%$ MCM-130-APB/APB-BPDA nanocomposite film These micrographs also showed a high level of MCM-130-APB organoclay dispersion in this $8 \%$ clay/APB-BPDA film. The lower magnification micrographs showed that a homogenous dispersion was reached and few agglomerates were present. The micrographs all show a larger number of particles in the frame than the micrographs 
of similar magnification with 3 and 5\% MCM-130-APB in APB-BPDA. This effect is related to the concentration but there also seemed to be better dispersion in these films as well. The higher magnification micrograph shows a large number of individual clay particles indicating that there may be slightly better dispersion than in the 3 and $5 \%$ clay loaded films. The average size of the particles in the micrograph taken at $22000 \mathrm{x}$ magnification are smaller than those in the $3 \%$ and $5 \%$ loaded film as well. In all three films in which MCM-130-APB was used as the organoclay, high levels of clay dispersion were reached and it appeared from the TEM micrographs that higher concentrations of the organoclay are possible while maintaining good dispersion.

The experiment described above was repeated with MCM-BAOD organoclay used in the place of MCM-APB organoclay. Hybrid solutions were prepared with these organoclays and films that were prepared from the solution were examined with TEM. Preparations in which 3, 5, and 8\% of MCM-130-BAOD were placed in APB-BPDA were examined with TEM. The TEM micrographs are shown below in Figure 29.
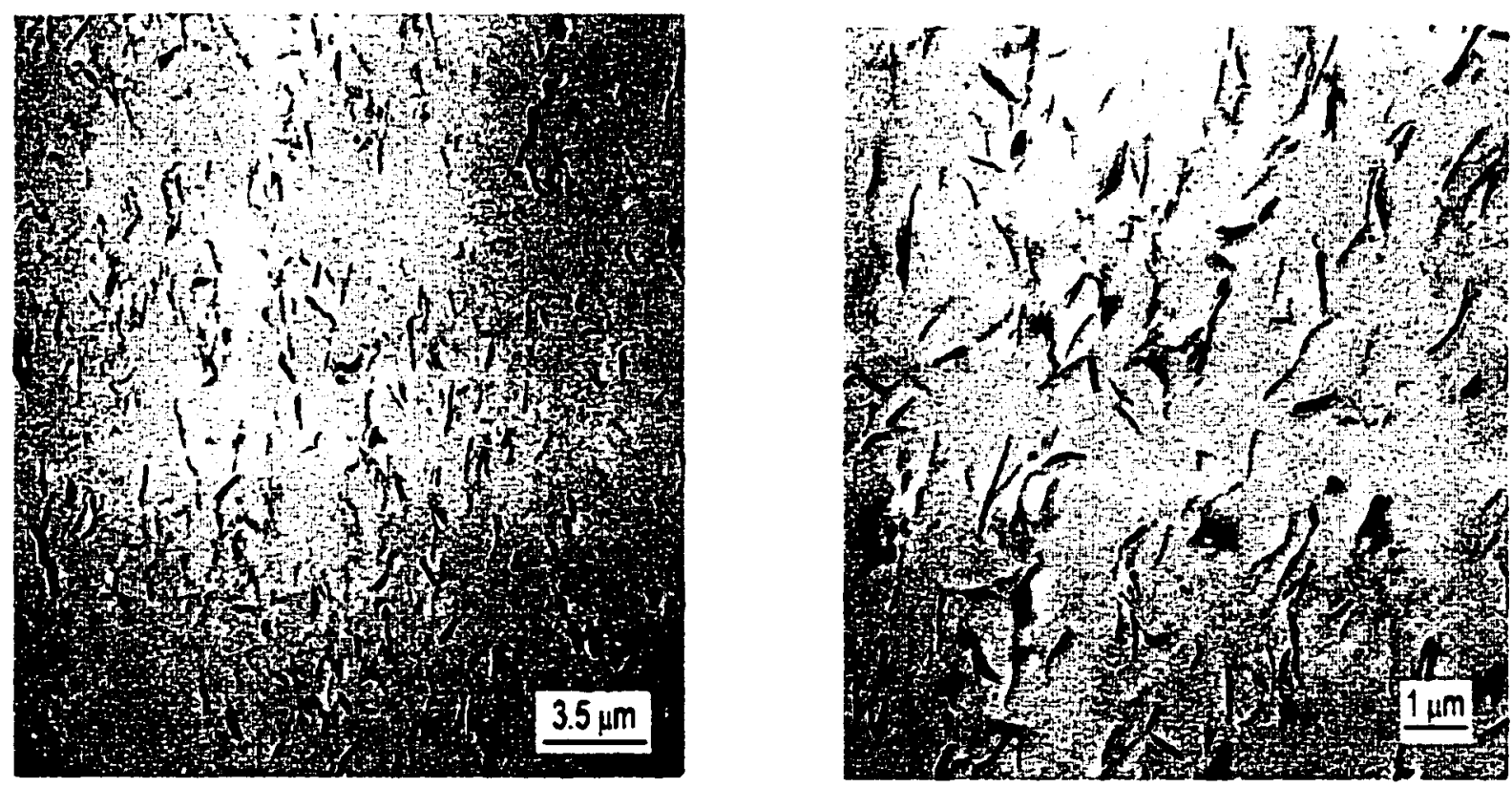


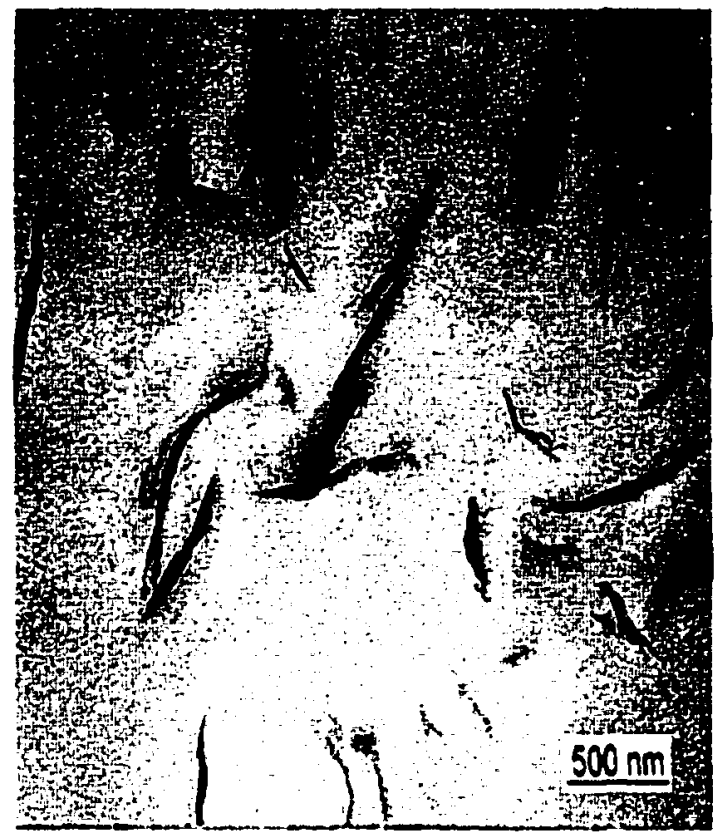

Figure 29. TEM micrographs of 3\% MCM-130-BAOD/APB-BPDA nanocomposite film These micrographs show that the dispersion was not at the high level reached in the MCM-130-APB loaded films. Although the clay was not as well dispersed as the MCM-130-APB organoclay, the fact that there were no large agglomerates indicated that there was good clay dispersion in these films. There was even distribution of the clay in the lower magnification micrographs, but there were fewer individual particles than in the micrograph of the 3\% MCM-130-APB film taken at the same magnification. More polymer area was present between each clay particle. The higher magnification film showed that the particles ranged from $300 \mathrm{~nm}$ to $1500 \mathrm{~nm}$ in length and that most of the particles were in small bundles.

The same organoclay was used in the preparation of 5\% MCM-130-BAOD/APBBPDA PAA solutions and films. The polyimide films were examined with TEM. The TEM micrographs for this sample are presented below in Figure 30. 

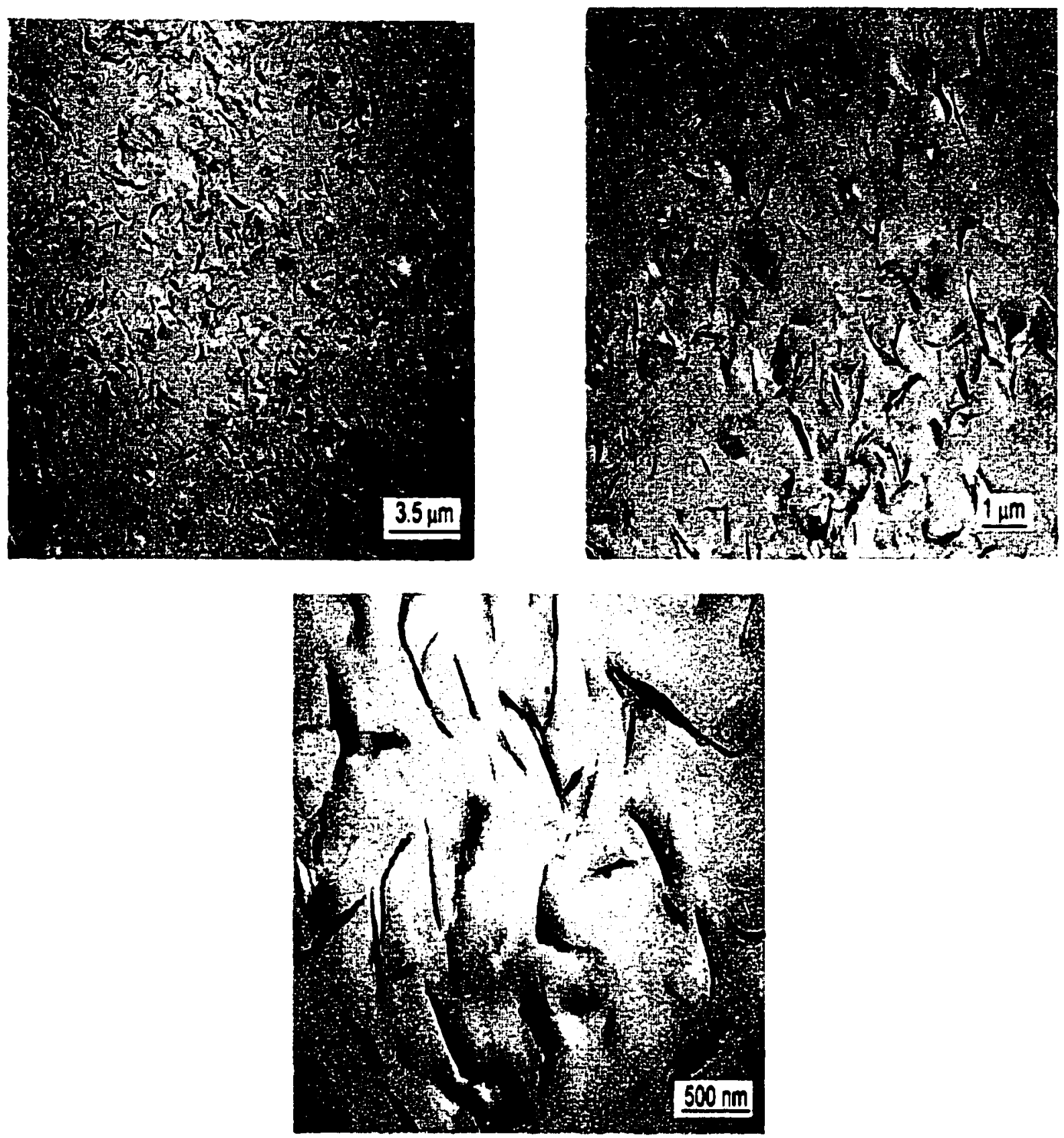

Figure 30. TEM micrographs of 5\% MCM-130-BAOD/APB-BPDA nanocomposite film There was an increase in the number of particles in the micrographs obtained on this film but this is most likely due to the increase in concentration. The dispersion may have increased slightly in this sample, as there are some individual clay particles in this film. 
Again the concentration was increased to $8 \%$ clay loading in the polymer film and TEM micrographs were taken of the hybrid material. The micrographs are shown in Figure 31.
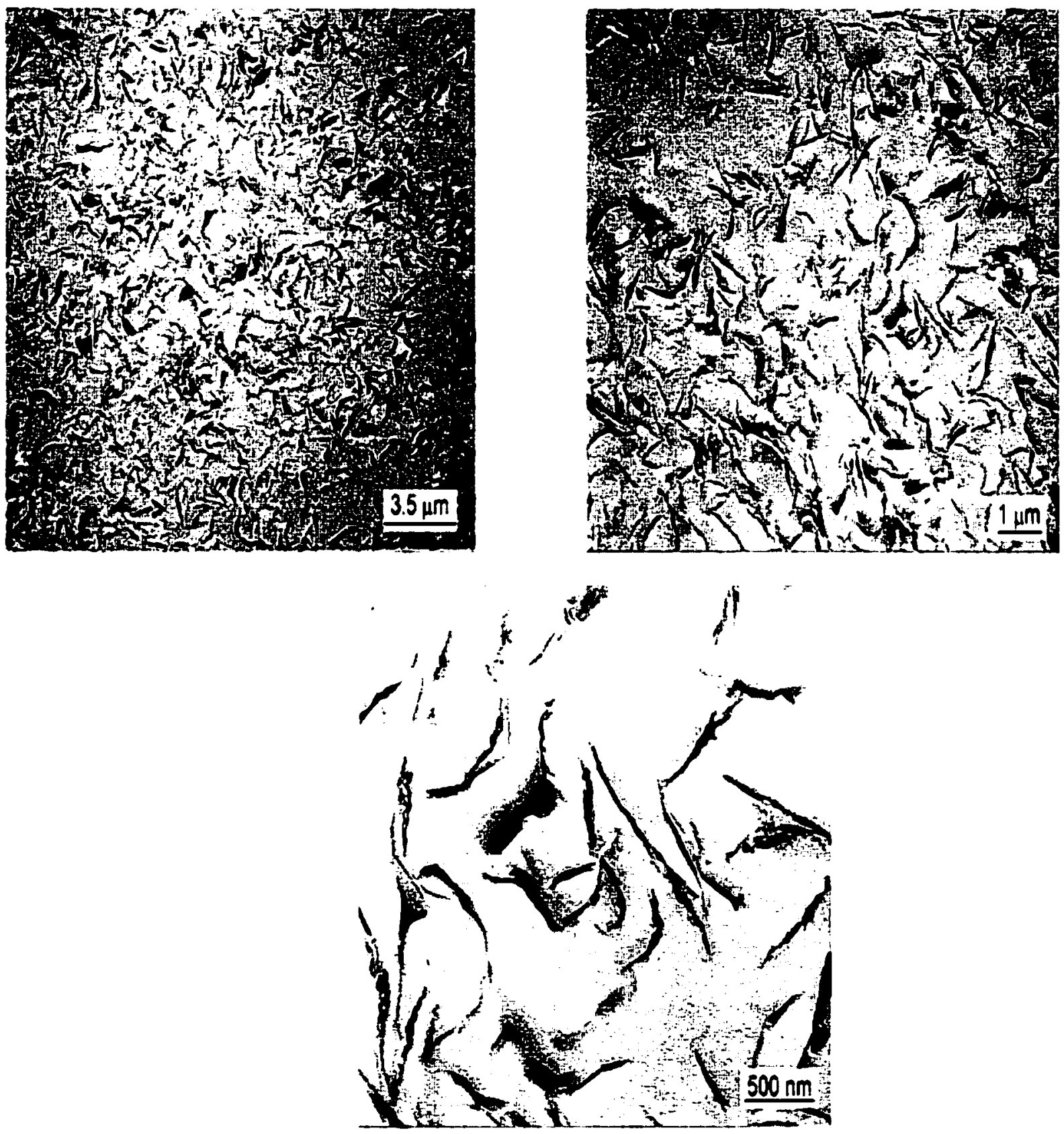

Figure 31. TEM micrographs of 8\% MCM-130-BAOD/APB-BPDA nanocomposite film 
The lower magnification films do show that there is even dispersion of clay in these films and that there are no areas of large clay agglomerates. The higher magnification micrograph also shows that there are some larger clay particles than in the $3 \%$ and $5 \%$ film and are obviously agglomerates of a small number of clay particles. The clay at this loading level seems to be a little less dispersed than in the lower concentrated films.

In all these MCM-130-BAOD films there was good dispersion of the clay but the clay was not all exfoliated. There are some exfoliated or single clay particles in these films but some of the particles were of larger dimensions.

In preparing the films with various organoclays there seemed to be a relationship between the appearance of the film and the charge that was on the clay. It was suspected from visual inspection that the lower charged clays (MCM-140 through MCM-170) contained more agglomerates than the more highly charged clays. This was confirmed with TEM. The TEM results for MCM-170-APB and MCM-170-BAOD organoclay in APB-BPDA at a 3\% loading level are presented in Figures 32 and 33 respectively. 

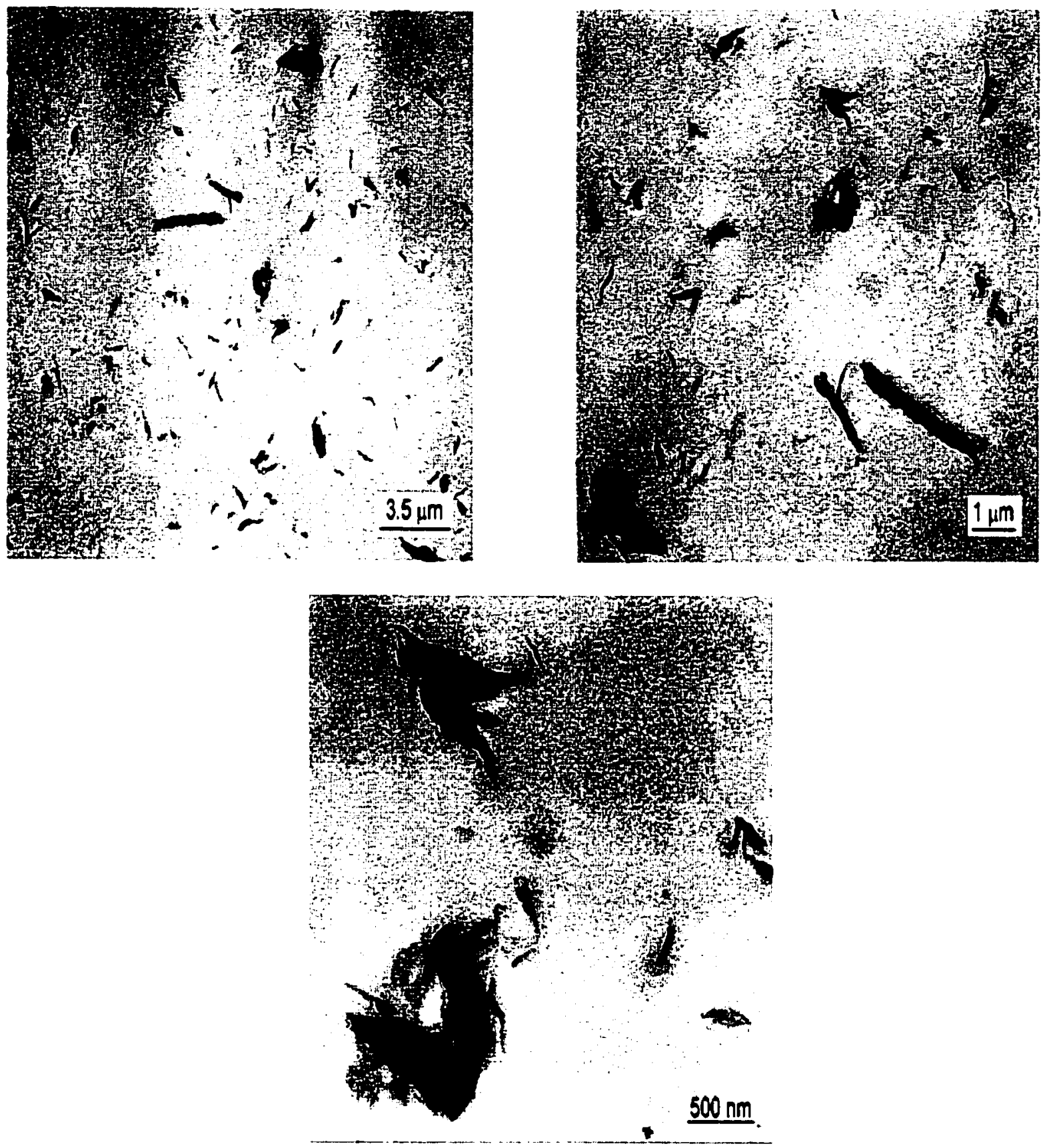

Figure 32. TEM micrographs of $3 \%$ MCM-170-APB/APB-BPDA nanocomposite film 

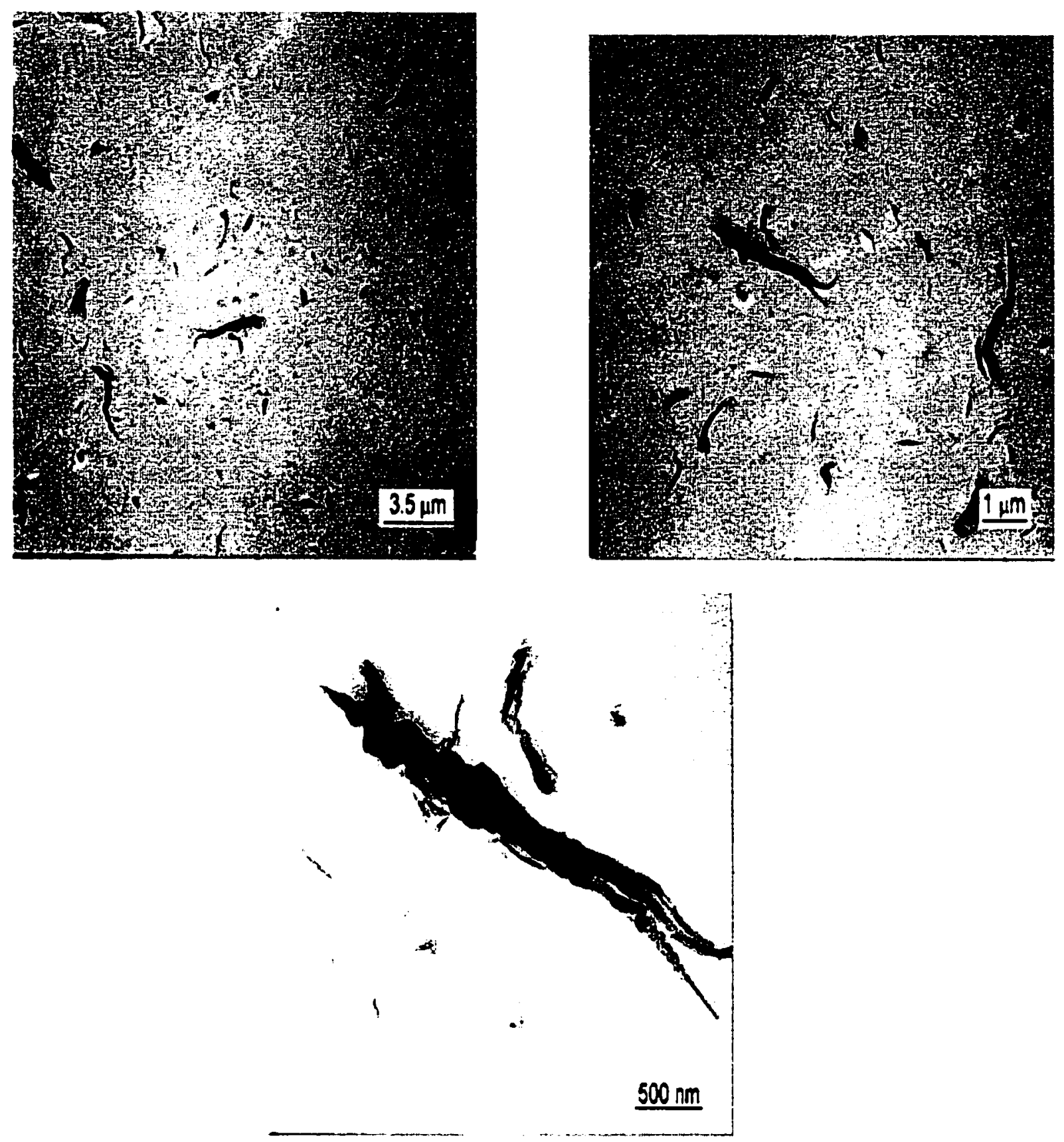

Figure 33. TEM micrographs of 3\% MCM-170-BAOD/APB-BPDA nanocomposite film

The film prepared with MCM-170-BAOD organoclay was similar in appearance to the MCM-170-APB film. In both of these films the clay particles were very poorly dispersed and there were large areas of the film that had no clay particles in them. There are few single clay particles in these systems and most of the clay exists in large 
agglomerates. The agglomerates present in the MCM-170-APB/APB-BPDA film are as large as $1 \mu \mathrm{m}$ wide and a couple of microns long. The TEM micrographs show that these systems had clay that was poorly dispersed which is consistent with the visual characterization performed on these films.

The TEM micrographs shown to this point were obtained from films of the two opposite extremes. The MCM-130-APB and MCM-130-BAOD organoclays contained some of the best dispersion in APB-BPDA. The MCM-130 clays were used to represent one extreme (high dispersion) while the other extreme where the organoclay is most poorly dispersed was shown in the MCM-170 organoclay/polyimide films. After characterizing the dispersion in these films it was very apparent that dispersion had some dependence on the charge on the clay surface. To further investigate this claim other organoclays with different charges on the surface were placed into the same polyimide and TEM micrographs were obtained. The percentage of clay was held constant at $3 \%$ in the polyimide/organoclay films so that they could be directly compared.

One of the organoclays that was placed in APB-BPDA was the organoclays that were fully charged. Films prepared with these FCM-APB and FCM-BAOD organoclays were visually clear. The TEM micrographs for the FCM-APB and FCM-BAOD organoclay/polyimide nanocomposite materials are presented below in Figures 34 and 35 respectively. 

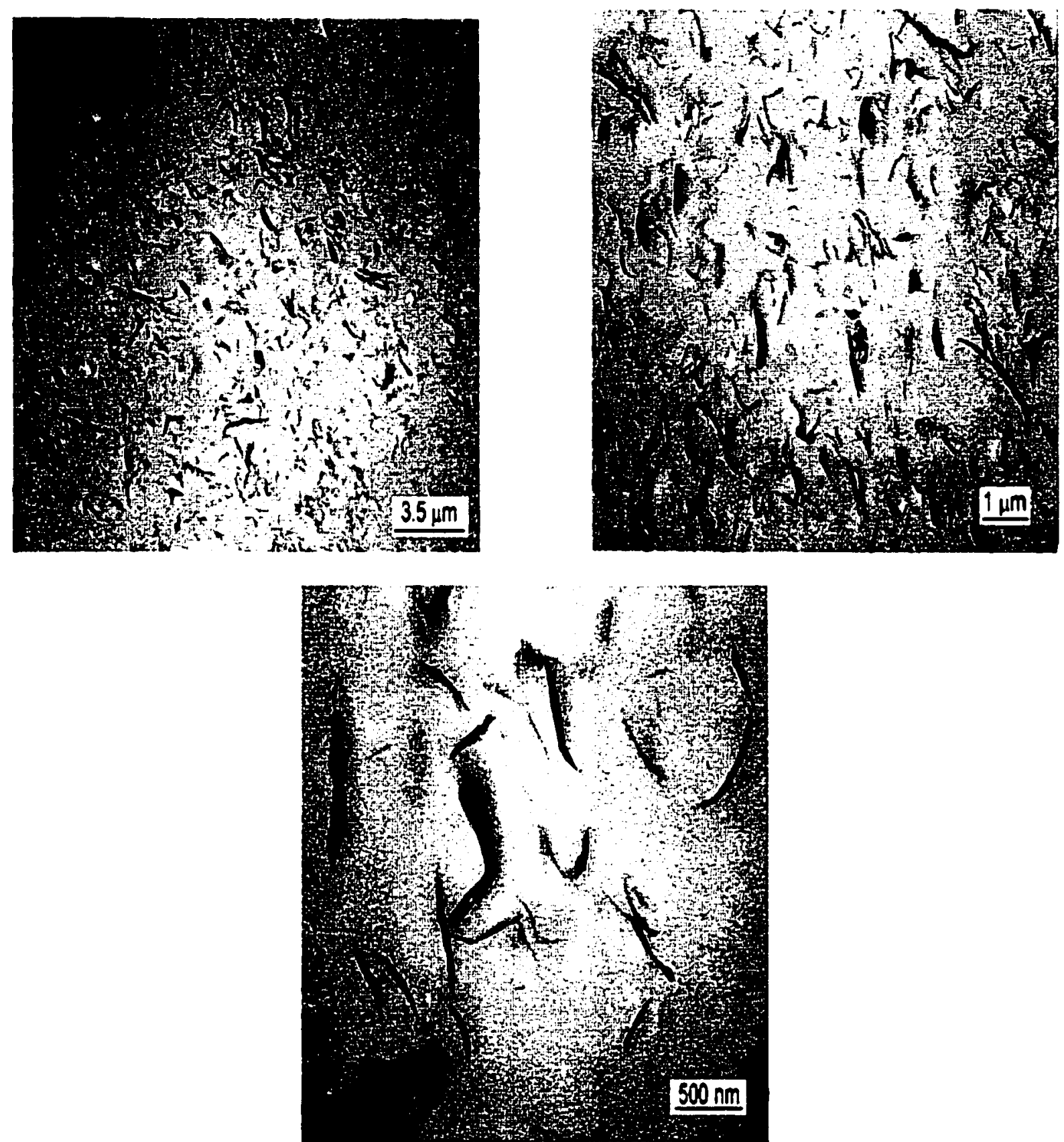

Figure 34. TEM micrographs of 3\% FCM-APB/APB-BPDA nanocomposite film

These micrographs of FCM-APB organoclay in APB-BPDA show that there are some clay agglomerates in this system. In the micrograph at $7200 \mathrm{x}$ magnification the agglomerates are clearly seen. There are more of agglomerates seen in these films than in the MCM-130-APB 3\% film. The agglomerates were not very large and consist of only a 
few particles of clay. The micrograph at higher magnification shows that there are some single clay particles but there were not as many as in the MCM-130-APB film. The dispersion of the clay in this film seems to be intermediate in the order of dispersion levels from the worst MCM-APB film (MCM-170-APB) to the best (MCM-130-APB).
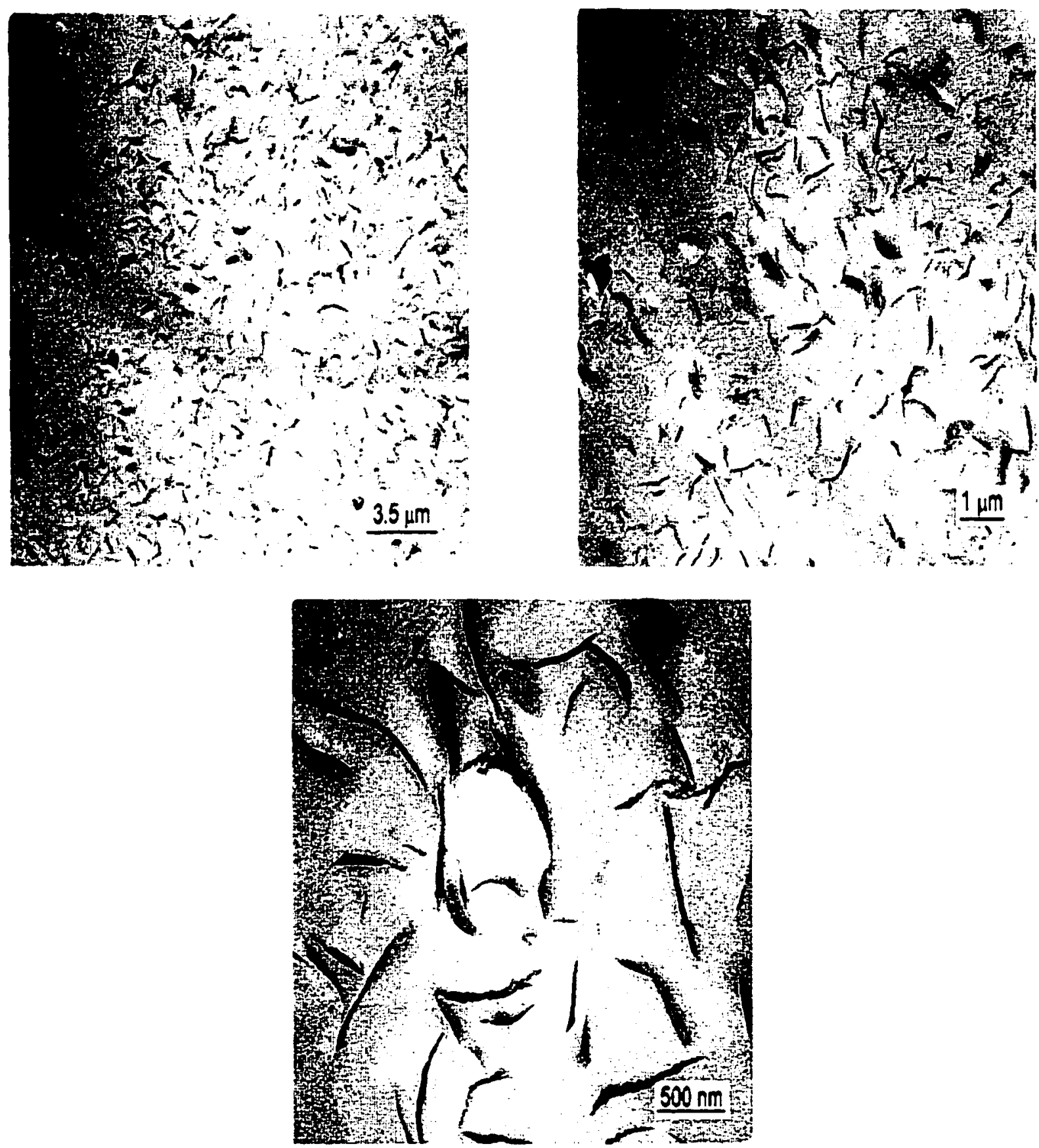

Figure 35. TEM micrographs of 3\% FCM-BAOD/APB-BPDA nanocomposite film 
The dispersion of clay in this FCM-BAOD material is very good and may be represent the best dispersion of the BAOD organoclays. There are some agglomerates in these films but the amount of particles in all the films seems to be greater than all other films prepared from BAOD organoclays. This result was surprising because it was thought that the MCM-130 clay would contain the highest level of clay dispersion in the APB-BPDA polyimide matrix. The MCM-130-BAOD clay did perform well but the TEM micrographs shown here do indicate that there was equal or better clay dispersion in this FCM-BAOD material.

To further study the relationship between the charge on the clay surface and the dispersion of the clay in a polyimide matrix, more clays were examined. It was thought that the clay that dispersed to the highest level was the MCM-130 clay. It was important to examine films that contained clays that were very close to the charge of the MCM-130 clay. This was done to see how sensitive dispersion was to surface charge. For this test the MCM-120/APB-BPDA and MCM-140/APB-BPDA preparations were examined. The TEM micrographs for MCM-120-APB and MCM-120-BAOD organoclays in APBBPDA are shown in Figures 36 and 37 respectively. Again the clay concentration is 3\% in these materials and three micrographs at different magnifications are presented for each organoclay/polyimide preparation. 

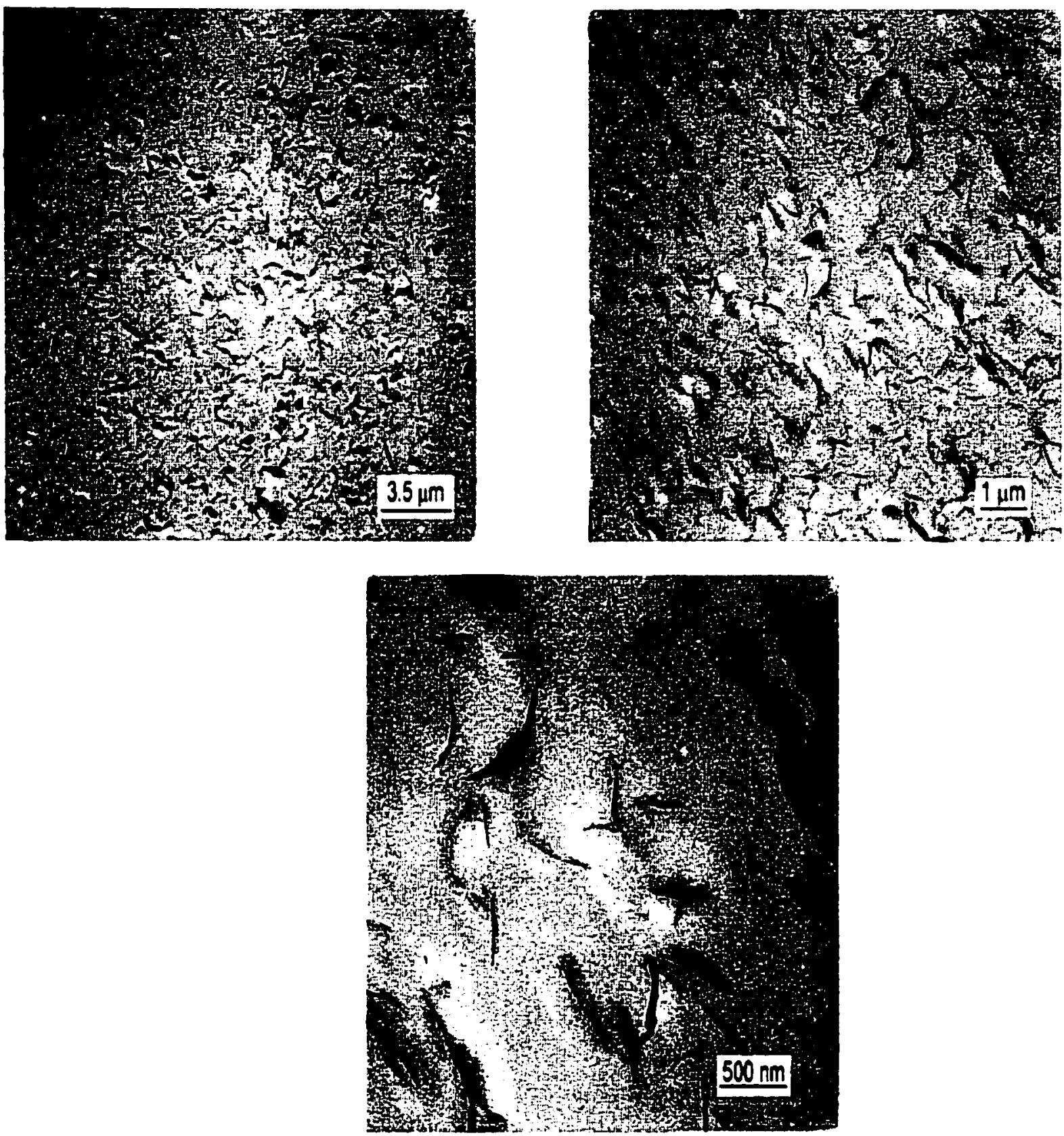

Figure 36. TEM micrographs of 3\% MCM-120-APB/APB-BPDA nanocomposite film

The micrographs shown here for the MCM-120-APB/APB-BPDA nanocomposite materials are similar to the MCM-130-APB micrographs and could almost be considered as well dispersed. The amount of distinguishable clay particles in the lower magnification films indicates that there are few particles that are agglomerated. The higher magnification film also shows that there are single clay particles and that there is a 
relatively low occurrence of agglomeration. In figure 37 micrographs are presented of a film with clay with the same charge on the surface but the BAOD ion was used instead. A high level of dispersion is seen in this film as well. The dispersion in this film was equal to or better than in the other polymer films prepared with the BAOD organoclay. The area of the film where no clay is present is small and the amount of agglomerates is low. The determination of what layer charge yielded the best dispersion when using BAOD as the surfactant has proven to be difficult. The FCM-BAOD, MCM-120-BAOD, and MCM-130-BAOD organoclays appear to have similar dispersion characteristics.
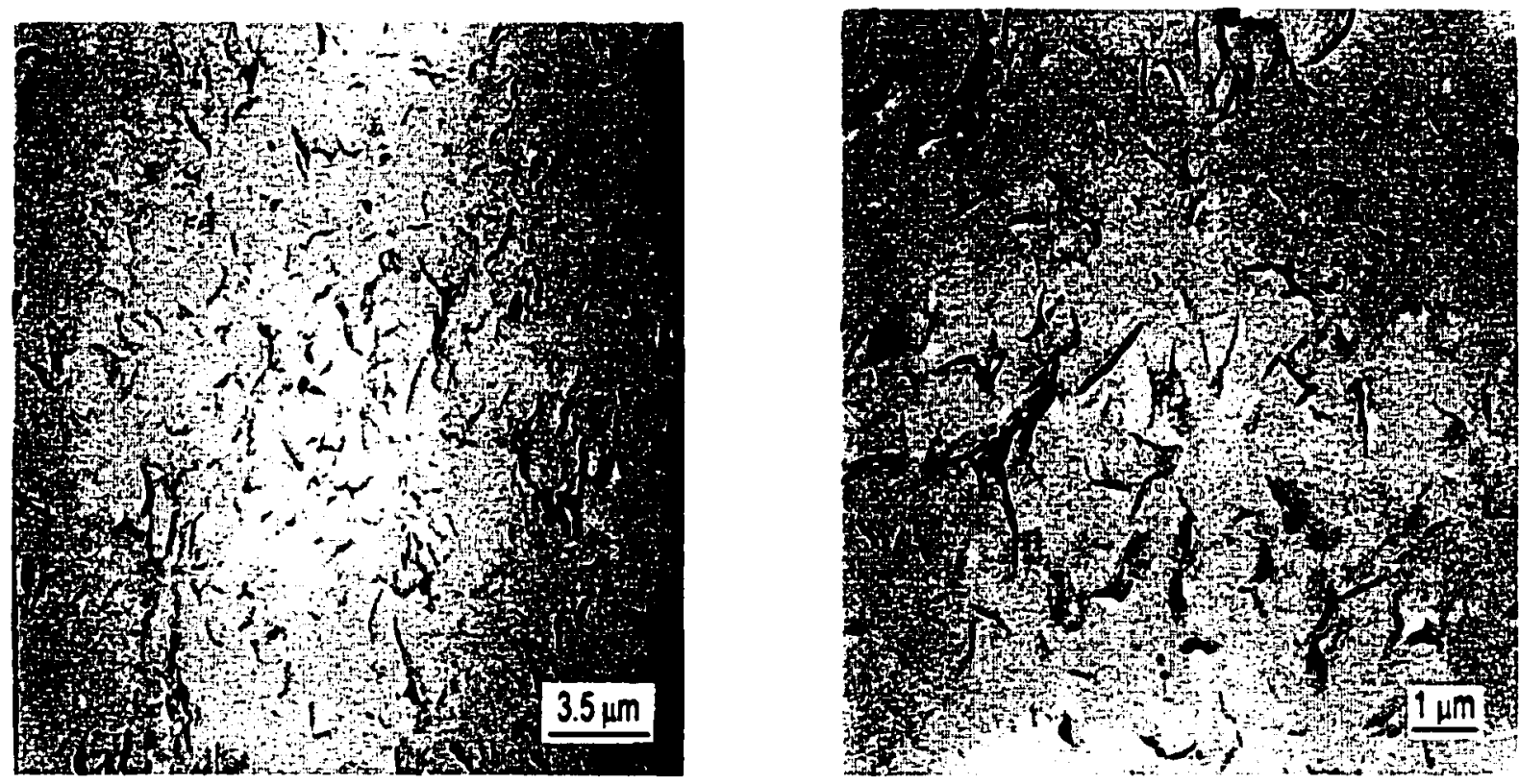


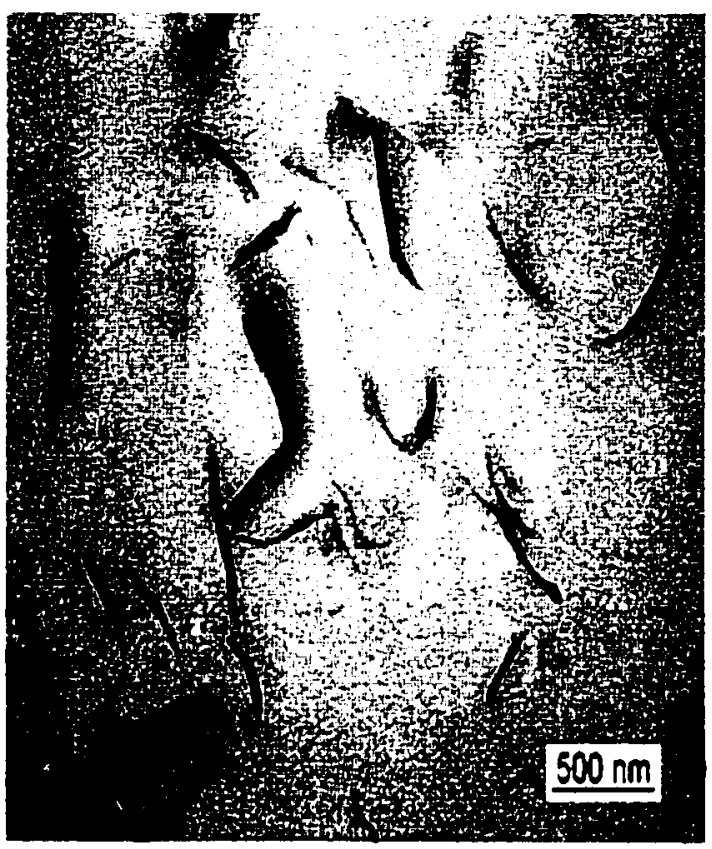

Figure 37. TEM micrographs of 3\% MCM-120-BAOD/APB-BPDA nanocomposite film The MCM-140 organoclays were examined for their dispersion properties in APB-BPDA polyimide. Films were prepared from MCM-140-APB and MCM-140BAOD organoclays and TEM micrographs for these films are shown in Figures 38 and 39.
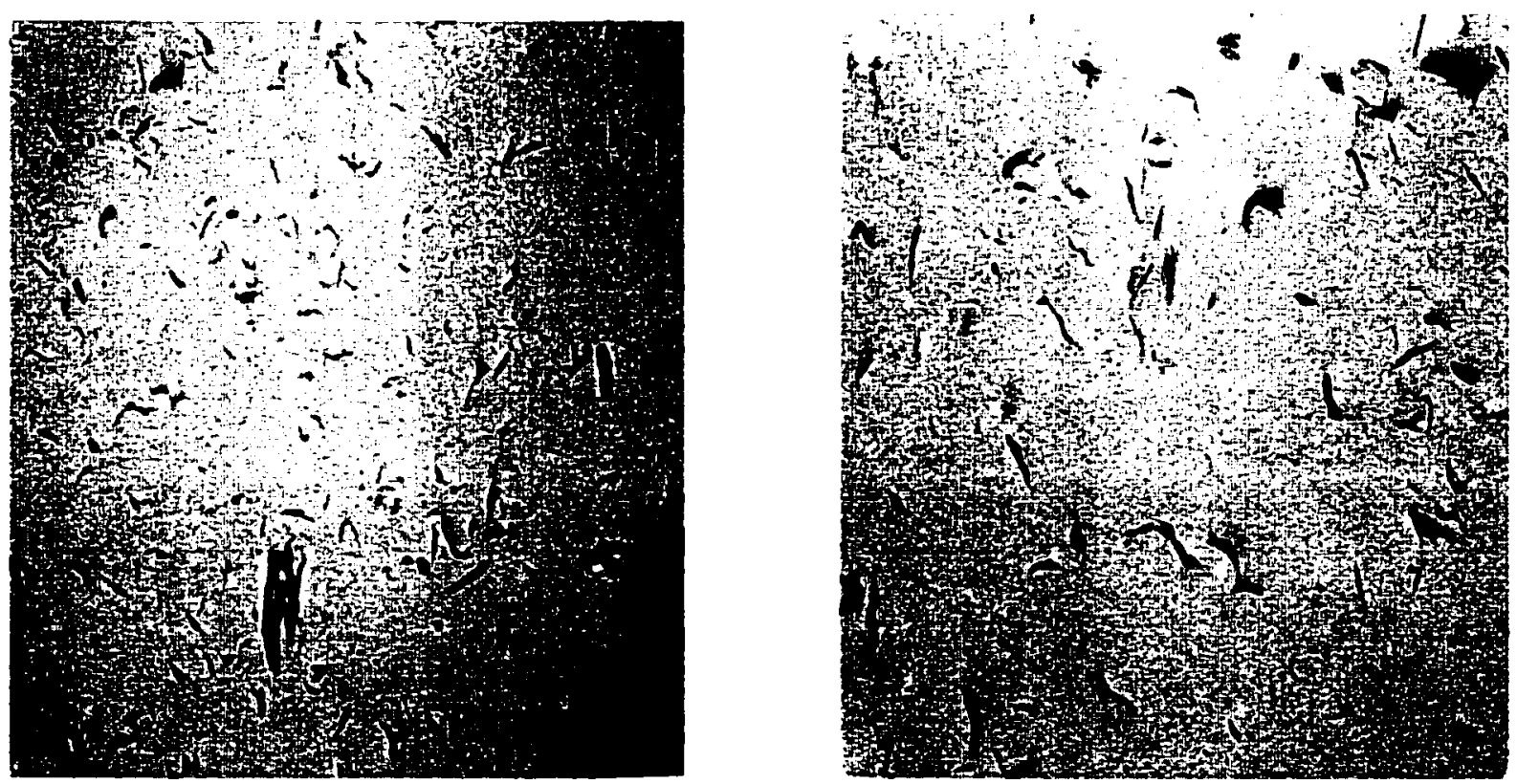


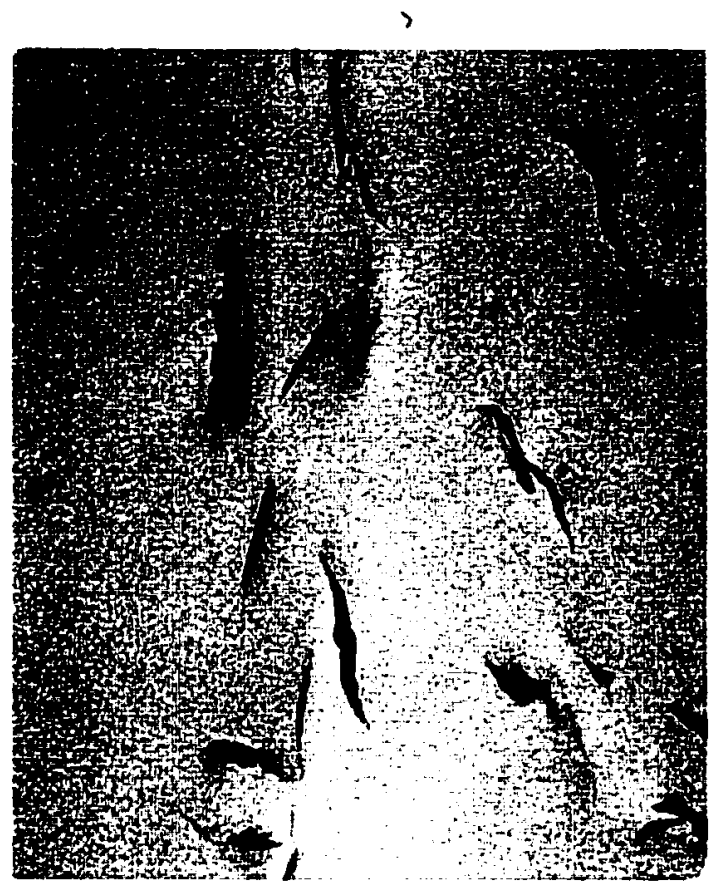

Figure 38. TEM micrographs of 3\% MCM-140-APB/APB-BPDA nanocomposite film

These TEM micrographs of the film prepared with MCM-140-APB organoclay showed that the dispersion level of the clay in these materials was relatively low. There are large areas of the film where no clay is present and there are agglomerates in the micrographs. The clay exists in bundle of particles and there are hardly any single clay particles. The fact that there are a lot fewer particles in these micrographs than in the MCM-130-APB film and the other well-dispersed films indicated that the MCM-140APB organoclay did not disperse as well. This result has to be a result of the layer charge as everything else was held constant.

The micrographs in figure 39 show micrographs of MCM-140-BAOD in APBBPDA. These micrographs show that the same charge with a different ion produced better nanocomposites. There is still a relatively large amount of agglomeration in these films when compared to the FCM-BAOD, MCM-120-BAOD, and MCM-130-BAOD. The increased agglomeration beginning at this point shows quite conclusively that the 
charge on the surface of this clay was too low and that the dispersion is sensitive to the charge on the clay.
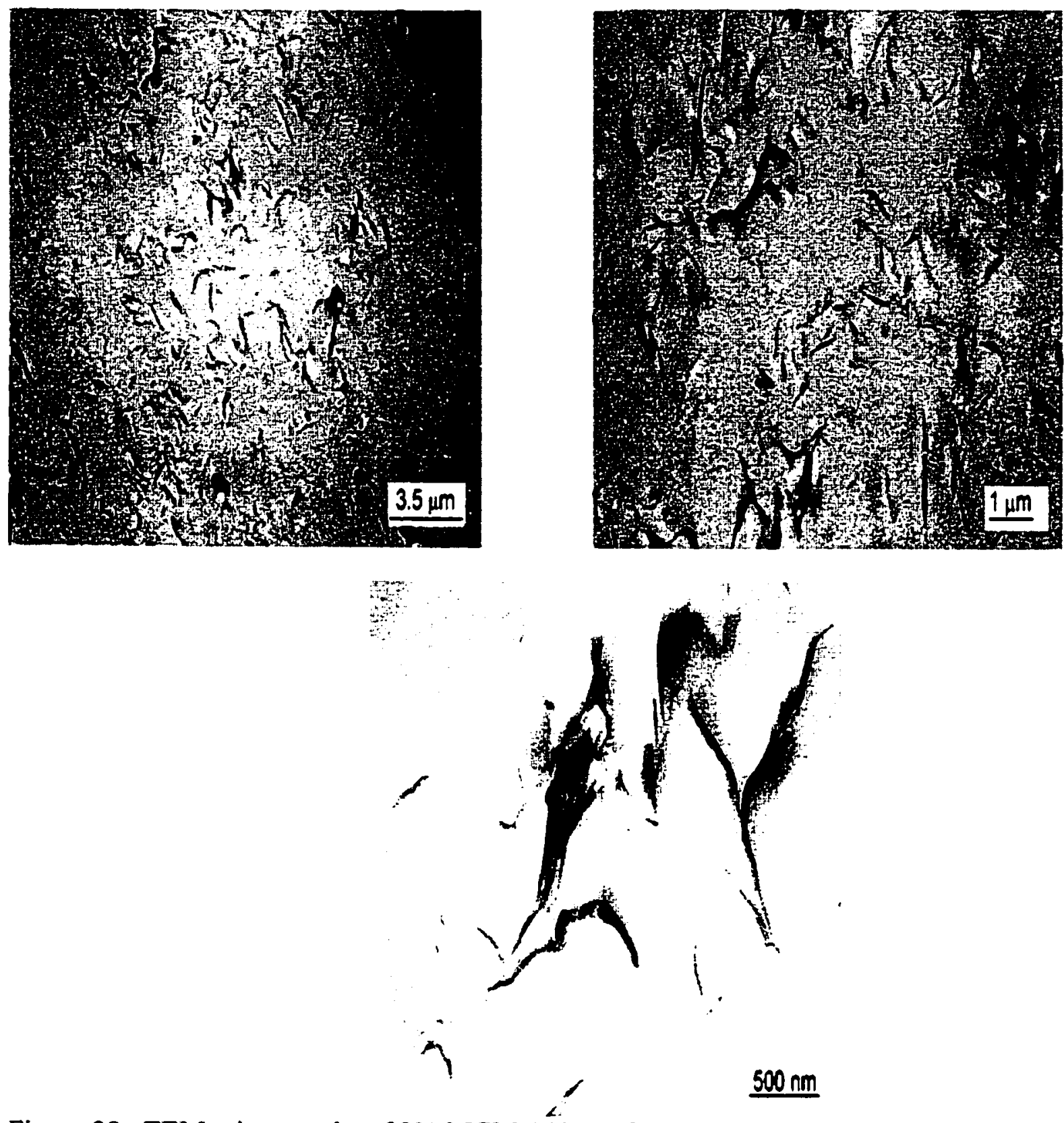

Figure 39. TEM micrographs of 3\% MCM-140-BAOD/APB-BPDA hybrid film 


\section{Conclusion}

Preparing polyimide/organoclay nanocomposites materials has presented many challenges. The obstacles presented when preparing this type of material are almost all associated with the thermal stability of the organoclay material. The organoclays that were typically used in the preparation of nanocomposites with other polymers cannot be used with the polyimides. This of course is a major challenge to replace the organoclays that are commercially available and that already have the desired swelling features and dispersability in polymer matrices. A whole new class of organoclays was needed. The new class of organoclays that was needed had to possess the same kind of dispersion properties and also needed to withstand the processing temperatures of polyimides. This was a tall order and it appeared that conventional clays and surfactants were not going to be able to fulfill the needs. For this reason aromatic surfactant ions were chosen for study and alterations were made to the clay as well.

The design and preparation of a novel organoclay began with choosing surfactant ions. The ions that were chosen for this work were high temperature aromatic moieties. For these aromatic moieties to be useful they must be able to be converted to a salt. It was thought that these ions should be as large as possible and contain the most amount of flexibility possible. The literature also pointed to the fact that these aromatic ions would probably work better if they had a place to tether the polymer chain to the surfactant. For these combined reasons the dihydrochloride salts of both APB and BAOD were chosen as the surfactant ions for this study.

After the ions were chosen the next steps were to both prepare the organoclay and to get it dispersed in both solvent and the PAA matrix. The preparation of the organoclay 
material had some critical features that needed to be examined including heating schedules, washing mechanisms, and drying methods. The correct method for preparing the organoclays was discovered and is presented above. These aromatic organoclays prepared from the Cloisite-Na+ clay and the chosen ions did have some good dispersion characteristics in both solvent and the polyimide, but the clays were not completely exfoliated. It was revealed that more alterations to the clay were needed in order to create quality polyimide/organoclay nanocomposites. The work done in Chapter IV with the differently charged clays indicated that the medium charge clays were better dispersed than their fully charged and reduced charge counterparts. A series of experiments were set up that would compare the charge on the surface of the clay and what role this would play in the dispersion characteristics of the clay. These experiments yielded some interesting results.

The preparation of organoclays with APB and BAOD ions with differently charged clays resulted in organoclays that were useful in nanocomposite preparation. In both series of clays there were organoclays that were better dispersed due to the charge on the surface. The increased dipersion did not manifest itself in the mechanical properties of the materials as might be expected. In the APB organoclays the characterization showed that the MCM-130-APB organoclay was the organoclay that performed the best. The clay was not $100 \%$ exfoliated but the clay was as well dispersed as any polyimide/organoclay prepared to date. This clay was used to prepare nanocomposite films with higher clay loadings and the clay retained a high level of dispersion at these higher loadings. The mechanical properties of these films showed an increase in the modulus while the strength and elongation decreased. The BAOD 
organoclays produced similar results. The characterization of these films showed that the charge on the surface also played a role in the dispersion of the organoclay, although the optimum charge was not as clear as in the case of the APB organoclay. The FCMBAOD, MCM-120-BAOD, and MCM-130-BAOD seemed to be similar in dispersion. It was difficult to determine which of these had the best dispersion but these were all much better than the further reduced charged BAOD organoclays. The MCM-130-BAOD organoclay was used in the preparation of 3,5 , and $8 \%$ organoclays and these films were tested for their mechanical properties. The results of this testing again showed an increase in modulus and decreases in both strength and elongation. These results of both of the organoclay systems indicated that these systems were superior to any polyimide/organoclay prepared to date and rivaled any of the systems reported in the literature.

The results from these experiments also revealed the fact that dispersion of the organoclays in polymer matrices were controlled by more factors than just the surfactant used and the mixing technique but also was a result of the charge on the surface of the clay. This is very important for future research in this area as another variable can be adjusted in order to create quality nanocomposite polyimide/organoclay materials. 


\section{Chapter $\mathrm{V}$ references}

1) D. Delozier, R. Orwoll, J. Cahoon, N. Johnston, J. Smith, and J. Connell. Polymer 2002;43:813.

2) W. Xie, Z. Gao,W. Pan, R. Vaia, D. Hunter, A. Singh, Polym. Matl. Sci. and Eng. Proc. 2000;82:284.

3) Y. El-Nahhal, S. Nir, C. Serban, O. Rabinovitch, and B. Rubin. J. Agric. Food Chem. 2000;48:4791.

4) J. Stevens and S.Anderson. Clays and Clay Minerals 1996;44:132.

5) W. Jaynes and G. Vance. Clays and Clay Minerals 1999;47:358.

6) H. Tyan, K.Wei, T. Hsieh, J. Poly. Sci., Part B, Poly, Phys. 2000;38:2873.

7) Tyan et.al., Chem. Mat. 2001;13:222.

8) H. Tyan, K.Wei, T. Hsieh. Chem. Mater. 1999;11:1942.

9) R. Vaia, E. Gianellis. Macromolecules 1997;30:8000.

10) J. Budjak, E. Hackett, and E. Gianellis. Chem. Mater. 2000, 2168.

11) J. Budjak and P. Komadel. J. Phys. Chem. B 1997;101:9065.

12) W. Jaynes and J. M. Bigham. Clays and Clay Minerals 1987;33:440.

13) J. Garcia, J. De La Campa, and J. Abajo. J. of Polymer Sci. Part A: Polymer Chemistry 1996;34:659.

14) J. Ladislaw, unpublished results. 


\section{Chapter VI}

\section{Conclusion}

The research presented herein was performed in an attempt to obtain polyimide nanocomposite materials with exfoliated clay particles. Accomplishing this task was not nearly as easy as was originally anticipated. Due to the unexpected level of difficulty associated with the exfoliation of clay particles in polyimide matrices the depth and the breadth of the research was more extensive than originally intended. The research had three major topic divisions and many subtopics within the larger areas. The major topics were simple mixing, in-situ polymerization with low temperature organoclays, and in-situ polymerization with high temperature organoclays. The original goal of obtaining a polyimide material with highly dispersed clay particles was reached and many important discoveries were made along the way.

The first natural division in the research encompassed all the materials that were prepared by simple mixing techniques. Different combinations of polymer and organoclay were examined. It was determined that the results reported in the literature using the simple mixing techniques were difficult to reproduce. Thus, alterations were made to the mixing technique found in the literature including sonication, melt mixing, and homogenization. The high shearing forces created by the homogenizer seemed to yield better clay dispersion in the polyimide matrix. This process yielded clear films that had similar mechanical properties to those normally encountered in well dispersed systems. Both aromatic and aliphatic surfactants were used to modify the clay surface in these experiments. The aliphatic clays dispersed more readily than the aromatic clays in these simple mixing experiments. The simple mixing technique was used at the 
beginning of the research with limited success but was quickly abandoned for a better preparation approach.

The second major division included all the materials that were prepared using the in-situ polymerization approach with low temperature organoclays. This preparation method yielded thin polyimide films with drastic improvements in clarity when compared to similar systems prepared with simple mixing. The low temperature organoclays that were used in these experiments were modified with organic salts that had long hydrocarbon chains attached to them. These organoclays dispersed to nanometer dimensions in some PAA/organoclay hybrid mixtures and in the polyimide films prepared from these solutions. Although there were some mixtures that were well dispersed using this approach, there were some PAA/organoclay solutions that were not clear indicating the clay did not disperse to nanometer dimensions in these mixtures. The reason for the poor dispersion of the organoclay in these systems is not understood, but it is speculated that dispersion may be related to the polarity and size of the monomers. In either case it was clear that the surfactants were degrading during the cure cycle of the PAA to the polyimide. This was determined from the darkening of the films after the cure cycle and was later supported by XRD and TEM. The surfactant degradation resulted in the clay that was not exfoliated collapsing. The resulting clay agglomerates were detrimental to the mechanical properties of the film and were not desired in the final product. Although the in-situ approach provided a better means of preparing nanocomposite materials, it was determined that a more thermally stable organoclay was needed. The research had progressed in the right direction with the in-situ approach but the focus of the project shifted to preparing high temperature organoclays. 
High temperature organoclays were considered those containing surfactants without aliphatic chains. The aliphatic surfactants were replaced with aromatic surfactant molecules in the high temperature organoclays. These were not conventional clay modifiers so basic research was performed in order to understand how the aromatic molecules would behave. A variety of aromatic surfactants were chosen and studied. The layer separation found in the clays with aromatic surfactants was smaller than typically encountered with the aliphatic organoclays. The lowered d-spacing was thought to be creating problems for monomer entry and subsequent polymer formation in the gallery with in-situ polymerization. In an effort to facilitate monomer entry into the inner layer during polymerization more of the clay surface was opened via charge reduction. At first the charge was reduced too far and no significant positive change was witnessed in the nanocomposite materials. It was suspected that the optimum surface charge was somewhere between the highest charge (FCM) and the lowest charge (RCM). An experiment aimed at proving this involved APB and BAOD surfactant molecules and a variety of charges on the clay surface. The optimum charge was found with the MCM130 clay when APB was the surfactant and at a slightly higher charge than this when BAOD was the surfactant. The resulting nanocomposites prepared with these optimally charged, high temperature organoclays possessed equal or better clay dispersion than nanocomposites prepared with the commercial organoclays. Although the strength and elongation properties did not improve in the films with better clay dispersion, the modulus did increase and the objective of exfoliating clay particles in a polyimide matrix was accomplished without the detrimental side effects of surfactant degradation. 
The research presented here represents a major step forward in both polyimide nanocomposite technology and clay nanocomposite technology in general. The concept of altering the concentration of surfactant on the surface of the clay can be implemented to other systems. This gives the researcher one more variable to adjust to create the best clay dispersion in polymer matrices. This is very important when considering the demand for nanocomposites and the limited amount of quality nanocomposites available. 
VITA

Donavon Mark Delozier

Born in Cumberland, Maryland, July 29, 1975. Graduated from Loudoun County High School in Leesburg, VA June 1993. Graduated from Emory and Henry College with a B.S. in Chemistry and a B.S. in Biology, 1993-98.

In August of 1998, the author entered the College of William and Mary as a graduate student in the Department of Applied Science. 